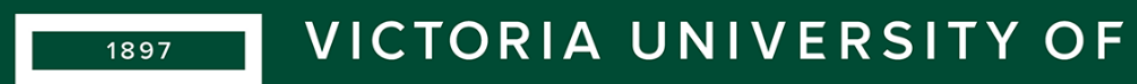 WELLINGTON TE HERENGA WAKA \\ NEW ZEALAND
}

\section{Electrochemical aptasensors: unravelling the critical interactions between aptamers, ligands, and electrodes}

A thesis submitted to the Victoria University of Wellington in fulfilment of the requirements for the degree of Doctor of Philosophy
Author:
Clement Sester
Supervisor:
Prof. Justin Hodgkiss
$2^{\text {nd }}$ Supervisor:
Assoc. Prof. Robin Fulton

Victoria University of Wellington

2021 


\section{Abstract}

Aptamers are synthetic nucleic acid single-stranded oligonucleotides that bind to a wide range of ligands, including cells, proteins, DNA strands, metal ions, and small molecules, with high specificity and affinity. Aptamers have also proven to be highly stable, readily adaptable to chemical modifications, and exhibit reversible binding. As a result, aptamer-based biosensors (aptasensors) are promising replacements for antibody-based biosensors in many applications, particularly for small molecule ligands. This thesis explores an aptamer that binds the drug methamphetamine, and its prospects when incorporated in an electrochemical (e-chem) signal transduction platform. Specifically, we examine the range of interactions between the aptamer and ligand, and with electrodes, and identify a number of challenges in generating robust echem aptasensors.

Due to their size and limited number of functional groups, further understanding of the aptamer-small molecule ligand interactions is required for the design of future aptasensors particularly the thermodynamics and structural information about the aptamer-ligand interaction. In fact, detecting small molecules with aptasensors can become challenging because target addition may induce little structural change, and therefore numerous nonspecific interactions may emerge as transduced signals from the biosensor. In this thesis, the combination of spectroscopic and calorimetric analytical techniques reveals a conformational selection binding model, in which binding is entropically driven, and the meth binds via hydrophobic and electrostatic interactions and only induces a modest structural change. This first-of-its-kind study is important for the selection and the design of the aptasensor transduction system.

Electrochemical (e-chem) aptasensors offer high inherent sensitivity and practicality as a signal transduction platform. Indeed, different e-chem aptasensor formats have been published before, including labelled and label-free sensors. In screening the viability of three commonly used methods - including labelled and label-free, as well voltammetric and impedance-based methods - we find that each of them suffers from instability of the aptamer-functionalised electrode. This instability compromises our ability to resolve real signals and prompted us to develop ways to understand - and suppress - this baseline drift.

The functionalization of the electrode is the critical step in terms of self-assembled monolayer (SAM) stability and SAM aptamer density. Consequently, different protocols of SAM 
formation were explored and evaluated with respect to stability. We find that instability arises from the uncontrolled arrangement of thiolated aptamers on gold electrodes (including aptamers lying down on the electrode), which is in turn affected by the density of aptamers that can be coupled to the surface. As a consequence, a new protocol is developed using disulfide aptamer pairs to increase the density of correctly tethered aptamers, and generate a stable SAM.

Because of the high sensitivity of electrochemical platforms, numerous spurious electrochemical signals may be produced, and controlled for in order to confirm a positive ligand-binding signal. The specificity of the aptamer-target interaction can be checked by testing the response with an interferent molecule, or by substituting the aptamer with a nonbinding nucleotide sequence. In this work, these control experiments reveal that target and interferent molecules interact directly (and in different ways) with the bare gold surface, as well as perturbing signals from the aptamer in ways that cannot be linked to a specific aptamerligand complex formation. Ultimately, these spurious signals compromise our ability to confirm a real binding signal.

The results from this work provide the first clear picture of how an aptamer binds to its small molecule target - which we find is entropically driven, and with only minor structural change induced in the aptamer stem. In addition, the label-free EIS measurements on aptamer SAM electrodes reveal the nature of instabilities, and reveal spurious signals that cannot be sufficiently suppressed at this stage. This knowledge highlights the difficulty in fabricating echem aptasensors, and will assist in overcoming challenges faced during research and commercialization of aptasensors area, as well as contributing new insights into troubleshooting, data acquisition, and data validation. 


\section{Acknowledgment}

After such an intense journey, it is the right time and place to acknowledge the people that surrounded and supported me during these last years. I remember the comparison between the $\mathrm{Ph} . \mathrm{D}$. journey and a rollercoaster mentioned by one person during the $\mathrm{Ph} . \mathrm{D}$. induction, in hindsight, this analogy describes perfectly the experience. Thanks to all the persons cited below the rollercoaster ride has been pleasant and highly rewarding.

First, I would like to thank my supervisor Professor Justin Hodgkiss who gives me the opportunity to come to New Zealand to be involved in this project. Special gratitude for encouraging my research and for helping me to develop my scientific skills. His advice and guidance have been priceless.

I express my gratitude to all the people from the "Hodgkiss group" for the pleasant and friendly working environment with scientific and non-scientific discussions. A particular shout-out to my two aptamers mates Anindita and Yasmin for the several scientific insights into my work that helped me growing my scientific curiosity and myself as a better scientist.

An enormous thank you to the whole staff of AuramerBio and all the person from the aptamer group of Janet Pitman. Being involved in your group meeting has been a significant help through my Ph.D., especially at the right time when I needed it.

Thank you to Paul, Karen, Shona, Joanne, and Shalen for the revision done on my writings.

I also want to acknowledge MBIE (Ministry of Business Innovation and Employment) for providing the scholarship which funded the tuition fees and living costs in New Zealand.

I am feeling grateful to everyone from the AM204 postgraduate office and mostly to everyone from the SCPS for providing a good mix of working and peaceful environment.

Furthermore, I wish to express a big thanks to my family and friends in France for all the calls and discussions that stimulate me to move forward every day during these three years.

Last but not least, thanks a lot to my wife, Joy for coming and experiencing with me these three unique years here in New Zealand. Without you, it would have been hard to go through that. 


\section{Research outputs}

\section{Conference poster}

"SAM investigation of aptamer-based electrochemical biosensor for methamphetamine detection”, AMN9 in Wellington, NZ. (10-14 February 2019)

\section{Conference presentation}

"Structural characterization of a methamphetamine aptamers family and target bound complexes", Aptamers2020 online in Oxford, UK. (3-4 September 2020)

\section{Commercialization programme}

Trans-Tasman Pre-Accelerator Programme organised by KiwiNet - March to May 2020 Mātauranga Pitau Ira Team - Revolutionary algal toxin detection tool 


\section{Abbreviations and common terms}

AB Assembling buffer

Aptamers Nucleic acid species with specific binding functions

Aptasensors Aptamer-based biosensors

AuNPs Gold nanoparticles

BWB Binding and washing buffer

CC Chronocoulometry

CD Circular dichroism

Cdl Double layer capacitance

CE Counter electrode

CV Cyclic voltammetry

DMSO Dimethyl sulfoxide

DNA Deoxyribonucleic acid

DPV Differential pulse voltammetry

dsDNA Double-stranded DNA

E2 17ß-estradiol

E-chem Electrochemical

EIS Electrochemical impedance spectroscopy

Ferri/ferro Potassium hexacyanoferrate (III)/Potassium hexacyanoferrate (II) redox couple

ITC Isothermal titration calorimetry

$\mathbf{K}_{\mathbf{D}}$ Dissociation constant

MCH 6-mercapto-1-hexanol

MDMA 3,4-Methylenedioxy methamphetamine 
Meth Methamphetamine

MPA 3-mercaptopropionic acid

NMR Nuclear magnetic resonance

NOESY Nuclear Overhauser effect

pdsDNA Partially double-stranded DNA

PBS Phosphate buffered saline

Rct Charge transfer resistance

RE Reference electrode

SAM Self-assembled monolayer

SELEX Systematic evolution of ligands by exponential enrichment

SG SYBR Green I

ssDNA Single-stranded DNA

SWV Square wave voltammetry

WE Working electrode 


\section{Contents}

Acknowledgment........................................................................................................................

Research outputs.......................................................................................................................................ii

Abbreviations and common terms ........................................................................................ iii

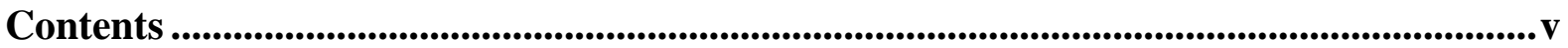

List of tables.............................................................................................................

List of figures.............................................................................................................................. viii

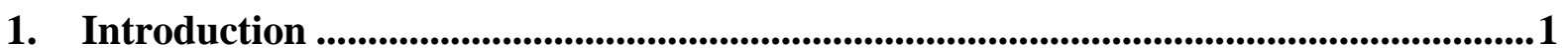

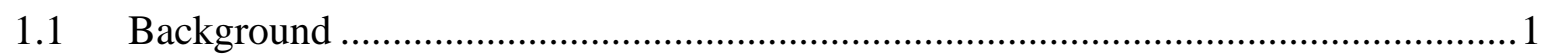

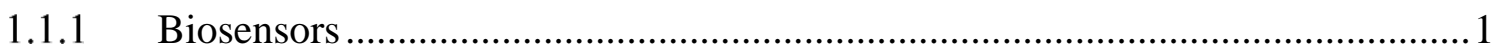

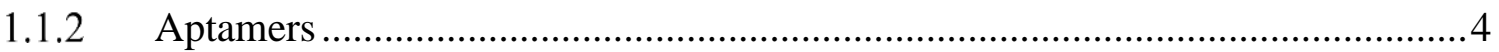

1.1.3 Aptamer-based biosensors: aptasensor technology ………………………........ 16

1.1.4 Aptamer characterization and aptasensors: challenges and opportunities ..........30

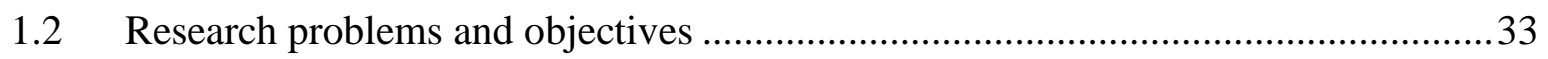

1.2.1 Methamphetamine problem and current method of detection ............................33

1.2.2 Research objectives and thesis overview ..........................................................

2. General methodology ............................................................................................38

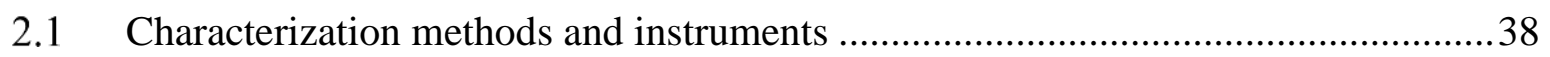

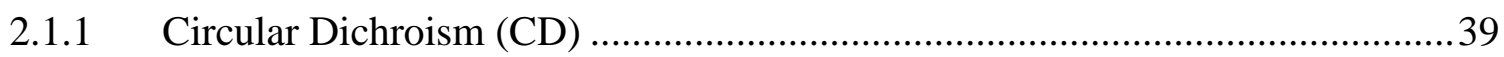

2.1.2 Isothermal Titration Calorimetry (ITC) ........................................................

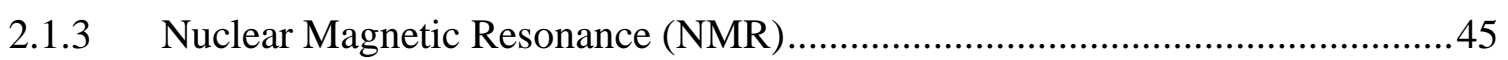

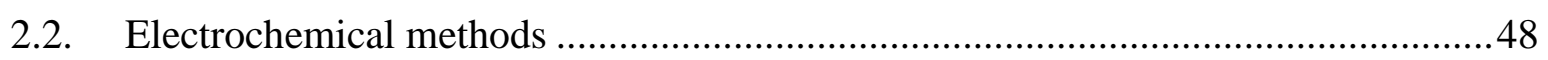

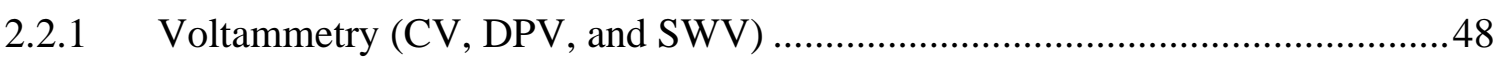

2.2.2 Electrochemical Impedance Spectroscopy (EIS) ..............................................55

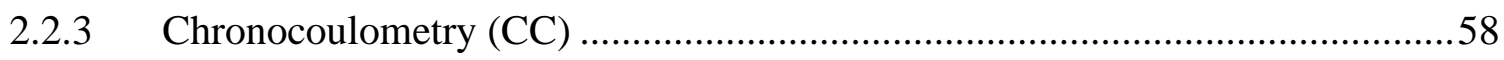

3. Unraveling the binding mode of a methamphetamine aptamer: a spectroscopic and calorimetric investigation.................................................................................................61

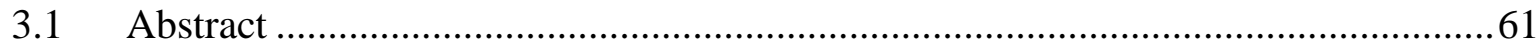

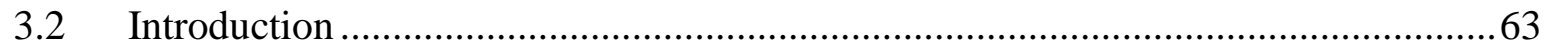

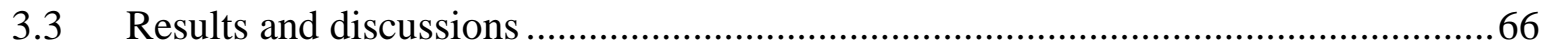

3.4 Chapter conclusion ...................................................................................... 80

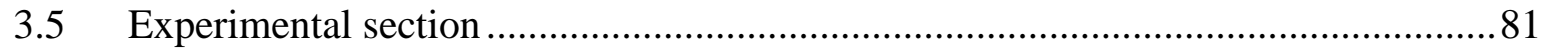

4. Screening the viability of three electrochemical aptasensor approaches....................84 


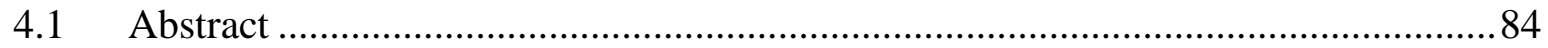

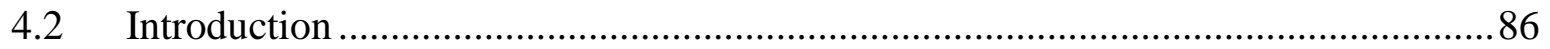

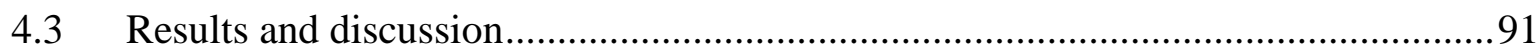

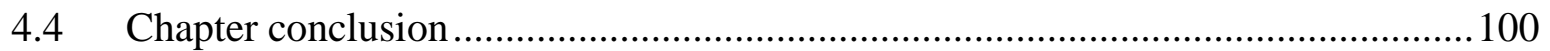

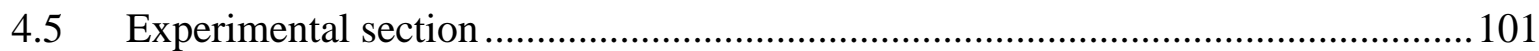

5. Self-assembled monolayer investigation via electrochemical impedance spectroscopy: stability and aptamer density optimization.............................................104

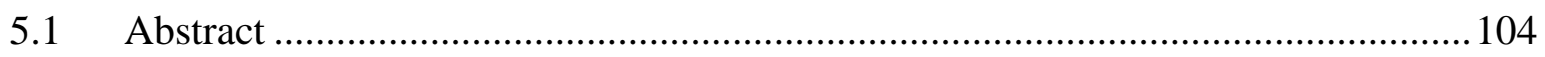

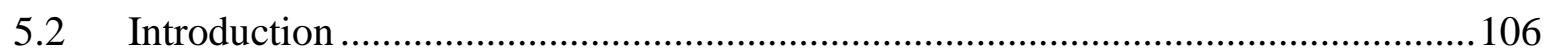

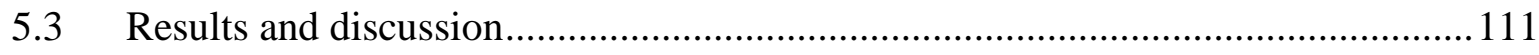

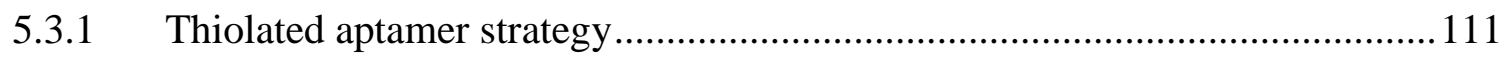

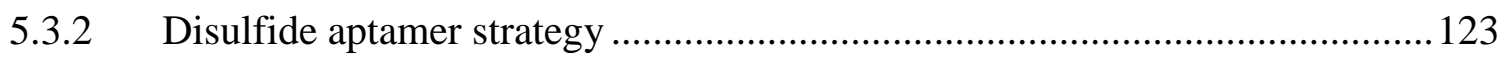

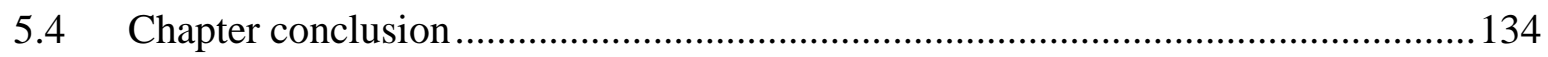

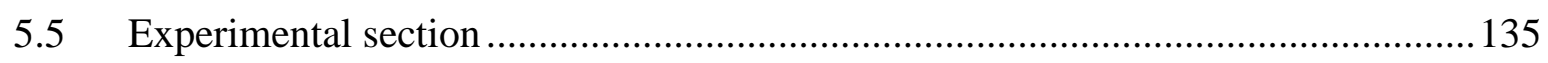

6. Investigating the range of response of the SAM upon methamphetamine

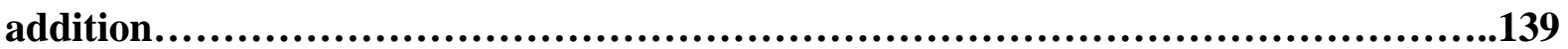

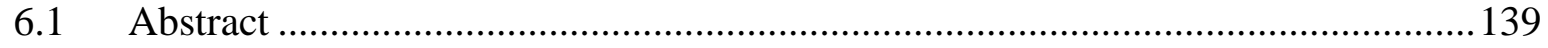

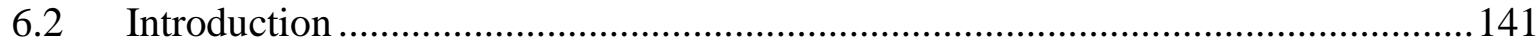

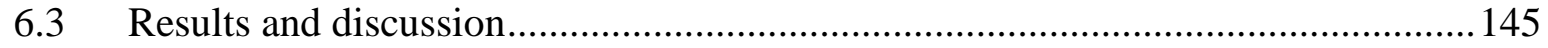

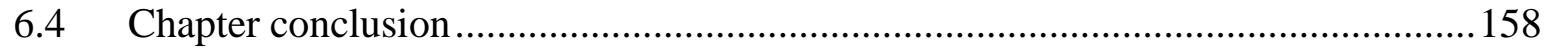

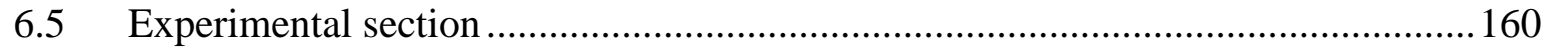

7. Conclusion and outlook .............................................................................................. 163

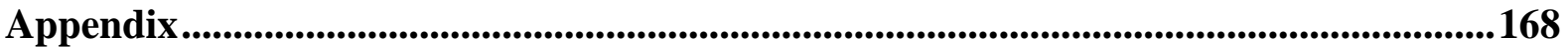

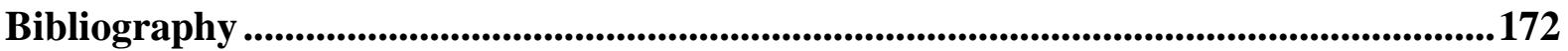




\section{List of tables}

Table 6.1. Nucleotides content, initial electron transfer resistance and aptamer density for the three different DNA strands used for SAM formation using the disulfide protocol. 156

Table A.1. DNA aptamer sequences used in the study. Buffer conditions are $2 \mathrm{mM}$ Tris- $\mathrm{HCl}$ $\mathrm{pH} 7.5,10 \mathrm{mM} \mathrm{NaCl}, 0.5 \mathrm{mM} \mathrm{KCl}, 0.2 \mathrm{mM} \mathrm{MgCl}_{2}$ and $0.1 \mathrm{mM} \mathrm{CaCl}_{2}$. 168 


\section{List of figures}

Figure 1.1 Schematic representation of the three parts of a biosensor platform.......................2

Figure 1.2 Representation of a DNA strand in two different dimensions. ..............................6

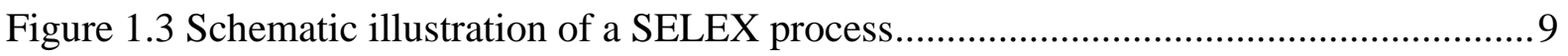

Figure 1.4 Representation of the binding event in two different dimensions........................ 11

Figure 1.5 Schematic illustration of three binding mechanism related to the aptamer-

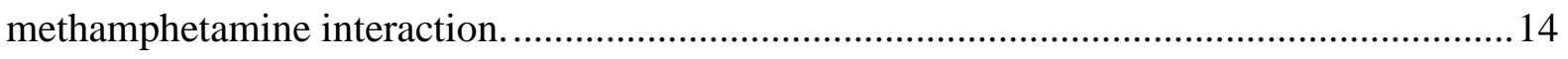

Figure 1.6 Schematic illustration of two sensing strategies employing aptamers and optical

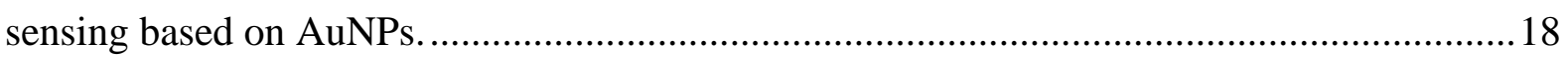

Figure 1.7 Schematic illustration of two sensing strategies employing aptamers and optical

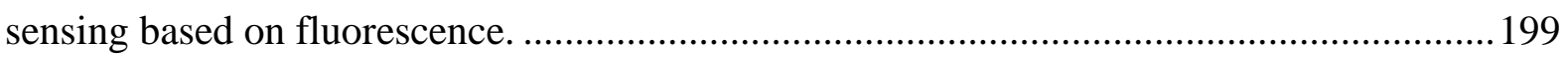

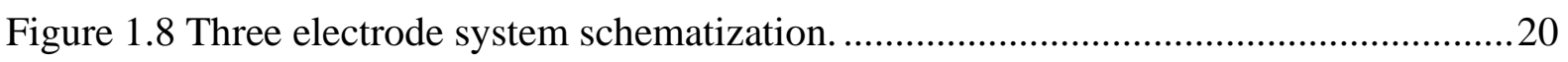

Figure 1.9 Schematic illustration of the interaction of an SH-ssDNA with a gold electrode..

Figure 1.10 Representation of two different protocol of fixation. .255

Figure 1.11 Schematic representation of two voltammetry sensing strategies employing a methylene blue and thiolated modified aptamer.

Figure 1.12 Schematic representation of two electrochemical label-free aptamer-based biosensor sensing strategies. .299

Figure 1.13 Skeletal representation of the methamphetamine molecule at pH7.5.. ......Erreur ! Signet non défini. 3

Figure 2.1 Schematic illustration of the analysis of an optically active molecule by circular dichroism. .40

Figure 2.2 Schematic illustration of the isothermal titration calorimetry instrument............444

Figure $2.3{ }^{1} \mathrm{H}$ NMR and NOESY spectrum of a DNA strand.

Figure 2.4 Cyclic voltammograms of bare gold electrode after electrochemically cleaning in $0.1 \mathrm{M} \mathrm{NaOH}$. .50

Figure 2.5 Typical cyclic voltammogram of bare gold electrode after electrochemically cleaning in $0.5 \mathrm{M} \mathrm{H}_{2} \mathrm{SO}_{4}$

Figure 2.6 Typical cyclic voltammogram of bare gold electrode in $5 \mathrm{mM}$ ferri/ferrocyanide solution. .53 
Figure 2.7 Schematic representations of potential vs time and current vs time for three voltammetry techniques.

Figure 2.8 EIS system with electrified electrode interface and equivalent electrical cicruit. 577

Figure 2.9 Nyquist plot representation. .588

Figure 2.10 (A) Chronocoulometric response for a DNA/MCH self-assembled monolayer without RuHex (Qdl) and with RuHex (Qtotal).

Figure 3.1 Binding models of bioreceptors in the context of aptamer-ligand binding. 644

Figure 3.2 Skeletal formula of protonated Meth, as dominant at $\mathrm{pH}$ 7.5. 666

Figure 3.3 SG assay for the Meth aptamers family. .677

Figure 3.4 CD experiments for the Meth aptamers family.

Figure 3.5 Aptamer-2-40mer representation with the hairpin structure encircled. .70

Figure 3.6 Watson-Crick base pairs A-T and G-C. .71

Figure 3.7 (A) Expansion of ${ }^{1} \mathrm{H}$ NMR spectrum of Aptamer-2-40mer showing three thymines imino protons peaks (B) 2D NOESY correlations between the three imino protons and the three adenine $\mathrm{C} 2$ protons showing $\mathrm{A}-\mathrm{T}$ base pairing. .72 Figure 3.8 The Aptamer-2-40mer and Aptamer-2-40mer - Meth solution recorded by ${ }^{1} \mathrm{H}$ NMR spectroscopy. .733

Figure 3.9 ITC results for the Aptamer-2-40mer and the mutated Aptamer-2-40mer. 744

Figure 3.10 Molecular docking of Meth against Aptamer-2-40mer. 799

Figure 4.1 The oxidation and reduction reaction of the methylene blue molecule. .86

Figure 4.2 Schematic representation of the three non-covalent interactions occurring between the methylene blue (MB) molecule and a partially double-stranded DNA.

Figure 4.3 The oxidation and reduction reaction of the ferricyanide/ferrocyanide couple. ..899 Figure 4.4 Schematic illustration of the formation of a self-assembled monolayer via a separated incubation of thiolated aptamer and $\mathrm{MCH}$ molecule.........................................911

Figure 4.5 Control of the SAM formation via two electrochemical techniques...................922 Figure 4.6 Schematic illustration of the methylene blue covalently attached to the thiolated aptamer strategy...... .933

Figure 4.7 The current signal dependency to the square wave frequency used for the SWV measurement is represented. Buffer and target response signals are plotted in function of the time. .944

Figure 4.8 Schematic illustration of the methylene blue non-covalently attached to the thiolated aptamer strategy.. .966 
Figure 4.9 Representation of the redox peak of the methylene blue molecule obtained via differential pulse voltammetry. Buffer and target response signals are plotted in function of the time.

Figure 4.10 Schematic illustration of the thiolated aptamer strand with the ferri/ferro redox couple strategy. 988

Figure 4.11 Electron transfer resistance in the function of the time for a buffer and a target experiment

Figure 5.1 Scheme of the different steps occurring during the self-assembly of thiolated DNA strand on gold electrode surface 1077

Figure 5.2 Schematic illustration of two e-chem cases of detection strategy on a gold electrode surface. 108

Figure 5.3 Schematic of the two-step thiolated aptamer and MCH molecule fixation on gold electrode surface.

Figure 5.4 EIS results for the two-step protocol with the 75 mer aptamer $-1 \mu \mathrm{M}$ incubated

Figure 5.5 Schematic representation of the fixation of four different blocking agents 1144

Figure 5.6 Schematic representation of the co-incubation protocol of thiolated aptamer and MCH molecule for the SAM formation on gold electrode surface. 1155

Figure 5.7 EIS results for the 75mer aptamer $-1 \mu \mathrm{M}$ aptamer incubated/100 $\mu \mathrm{M} \mathrm{MCH...1166}$ Figure 5.8 (A) Stability study for the thiolated 75 mer aptamer incubated at $5 \mu \mathrm{M}$ and $10 \mu \mathrm{M}$. (B) Stability study for the thiolated $28 \mathrm{mer}$ aptamer incubated at $5 \mu \mathrm{M}$ and $10 \mu \mathrm{M}$. 1177 Figure 5.9 Schematic illustration of the effect on the SAM fixation for a truncated aptamer (28mer). 1188

Figure 5.10 Investigation of the aptamer density and the electron transfer resistance for different thiolated aptamer incubated concentrations.

Figure 5.11 Investigation of the target incubation at $500 \mathrm{nM}$ and $50 \mu \mathrm{M}$ methamphetamine.

Figure 5.12 Schematic representation of the thiolated aptamer fixation approach and the disulfide aptamer fixation approach.

Figure 5.13 Ellman's experiment overall equation for the reaction of the acetylcysteine with the Ellman's reagent ..... 1244

Figure 5.14 Mild oxidation experiment of the cysteine thiol group into disulfide. 1255

Figure 5.15 Conversion of two thiolated aptamer into a disulfide dimer aptamer. 1266 
Figure 5.16 (A) Stability study for the disulfide 75 mer aptamer incubated at $5 \mu \mathrm{M}$ and $10 \mu \mathrm{M}$. (B) Stability study for the disulfide $28 \mathrm{mer}$ aptamer incubated at $5 \mu \mathrm{M}$ and $10 \mu \mathrm{M}$.

Figure 5.17 Investigation of the aptamer density and the electron transfer resistance for different disulfide aptamer incubated concentrations. 128

Figure 5.18 Schematic representation of the three different methods of SAM fixation tested. 130

Figure 5.19 Investigation of the target incubation at $500 \mathrm{nM}$ and $50 \mu \mathrm{M}$ methamphetamine. 131

Figure 5.20 Schematic representations of the disulfide 28mer aptamer and the disulfide 28mer $-\mathrm{MCH}$.

Figure 5.21 Investigation of the aptamer density and the electron transfer resistance for the disulfide $\mathrm{MCH}-28$ mer aptamer incubated concentrations. 133

Figure 6.1 Schematic of an e-chem aptasensor system on a gold electrode surface. 143 Figure 6.2 Two different experiments are represented, an overall signal induced by Meth addition occurs.

Figure 6.3 The methamphetamine adsorption in different BWB ionic strength on bare gold electrode and on $\mathrm{MCH}$ are monitored via EIS.

Figure 6.4 Skeletal formula of the predominant forms at pH 7.5 of the methamphetamine, the amphetamine, and the 3,4-Methylenedioxymethamphetamine (MDMA). 1500 Figure 6.5 Methamphetamine, amphetamine, and MDMA adsorption in BWB $2 \mathrm{mM}$ and BWB $20 \mathrm{mM}$ on bare gold electrode are monitored via EIS. 152 Figure 6.6 Schematic illustration of the Meth adsorption on gold surface and repulsion of MDMA from gold surface. 153

Figure 6.7 Comparison of the MDMA and the Meth addition on the disulfide Aptamer-2 system. 154

Figure 6.8 Meth sensing experiments for the Aptamer-2 - 28mer, the randomized - 28mer and the $\mathrm{E} 2-35 \mathrm{mer}$. 155

Figure 6.9 Schematic representations of the hypothetic explanation of the $R c t$ decrease and the Rct increase

Figure A.1. Aptamer2-40mer ${ }^{1} \mathrm{H}-\mathrm{NMR}$ spectrum. 1688

Figure A.2. Meth ${ }^{1} \mathrm{H}-\mathrm{NMR}$ spectrum in BWB 2mM Tris- $\mathrm{HCl} 90 \%-10 \% \mathrm{D}_{2} \mathrm{O}$. 169

Figure A.3. Partition results for Aptamer2-40mer obtained from RNAstructure. 169

Figure A.4. Initial Rct for different pure blocking agent SAM formed. 170 
Figure A.5. Ellman's reaction with cysteine.

Figure A.6. Thiol oxidation in disulfide mechanism with the dimethylsulfoxide (DMSO) molecule. 


\section{CHAPTER 1}

\section{Introduction}

\section{Chapter overview}

This chapter will introduce the concept of biosensing and the numerous approaches within this field. The use of biomolecules, specifically aptamers, as receptors in biosensing will be reviewed as well as the implementation of aptamers within sensing schemes. Research challenges and objectives of this dissertation will be established at the end of this chapter.

\subsection{Background}

\subsubsection{Biosensors}

The history of biosensors dates to 1906 when M Cremer demonstrated the relationship between the concentration of acid and the electric potential of the fluid. However, it wasn't until Griffin and Nelson between 1909 and 1922 who immobilised biological enzymes on aluminium oxide and charcoal that started the work on using biological receptors in sensing platforms. L.C. Clark 'known as the father of biosensors' work on oxygen detection in 1956 and subsequent work on glucose detection using amperometric enzyme electrodes in 1962 gave the field credentials for what is now the modern field of biosensors. ${ }^{1}$

A biosensor is defined as an analytical device for the detection, quantification, and monitoring of numerous chemical and biological compounds. ${ }^{2-4}$ It generally combines two functional components; a molecular recognition element (receptor) that selectively interacts with its target 
analyte (e.g., ions, DNA, antibodies, cells, and microorganisms) and a physicochemical transducer. ${ }^{5,6}$ The transducer converts the bio-recognition signal into a perceptible signal through the nature of the transducer used (electrochemical, electrical, optical, magnetic, masssensitive, or thermal signal) (Figure 1.1).

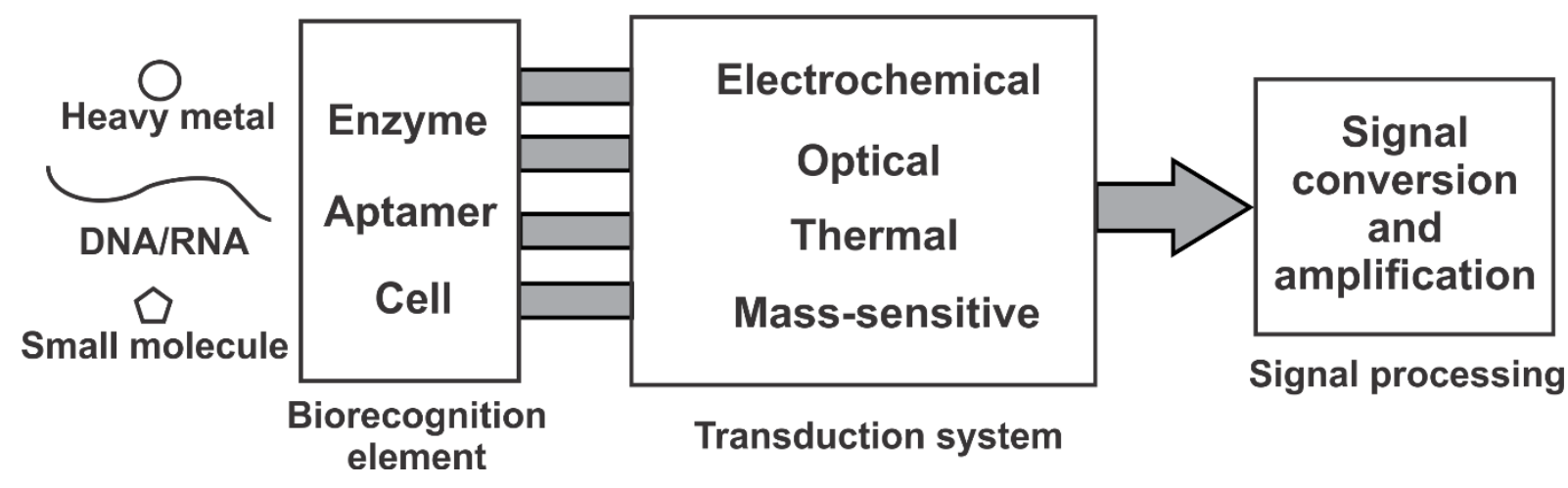

Figure 1.1. Schematic representation of the three parts of a biosensor platform including the analytes, biological recognition elements, transduction systems, and signal processing platforms.

Biosensors are constructed to measure target molecules of medical and environmental significance at a cost comparable to, or better than, traditional analytical techniques. ${ }^{7}$ Moreover, biosensor systems are mostly easy to use and portable. Numerous systems are commercially available such as glucose meter, ${ }^{8,9}$ portable blood analyzer, ${ }^{10}$ or pregnancy test. ${ }^{11}$

As shown in Figure 1.1, different biorecognition elements are used. ${ }^{6}$ Amongst them, two are compelling and therefore, mainly used for biosensing systems. Firstly, antibodies, the most popular class of biomolecules providing molecular recognition needs for a wide range of applications, have been around in biosensor technology for more than four decades. ${ }^{12,13}$ Antibodies are in vivo generated immune proteins generally characterised by their large Yshaped structure (roughly $150 \mathrm{kDa}$ and $10 \mathrm{~nm}$ size). Antibodies are produced to recognize specific antigens such as viruses, bacteria, proteins, or fungal cells, and are popular biorecognition elements due to their specificity, selectivity, and efficiency of binding. ${ }^{12}, 13$ However, the limitation of the antibody generation is that it needs a biological mammalian host for initial generation and optimal function. For small molecular targets, often the target molecule is conjugated to a small toxin to initial immune response needed for antibody generation in the host organism. This often affects the specificity towards the small molecular target. Additionally, in therapeutic applications, due to the larger size of the antibodies and biological origin, they initiate a negative immune response thus hindering the full potential use 
of antibodies. Furthermore, antibodies cannot be reversibly denatured and produced without batch to batch variations. ${ }^{14}$

Due to the aforementioned limitations, antibody use in therapeutics and diagnostics is being challenged by aptamers. ${ }^{14,} 15$ Aptamers are a newer class of bio-receptors derived from synthetically generated oligonucleotide library that is evolved using in vitro techniques.

\section{Aptamers as alternatives to antibodies}

The reasons why an aptamer offers several advantages over antibodies can be separated into the two different fields of application; biosensors and therapeutics. ${ }^{14-17}$ However, some aspects overlap between the two fields.

The in vivo production of the antibodies is problematic as it gives rise to batch-to-batch variations and raises ethical questions of the generation process that uses living animals. ${ }^{15}$ For the aptamer also considered as "chemical antibody", the generation process is an in vitro process. Chemical synthesis means it is less sensitive to batch variations and no living animals are used (except for the production of aptamers in vivo for therapeutic use). Furthermore, aptamer production is low in cost in comparison with antibody production and is not restricted to biological conditions. ${ }^{15}$

The antibody is a good biorecognition element for large molecules such as protein, cells, and bacteria. ${ }^{6}$ Indeed, it can create a strong affinity with these molecules of interest through different non-covalent interactions due to the rich functional group environment of both molecules. ${ }^{15,17}$ However, the antibody has a large rigid structure and a structural change would not be expected for antibody-molecule recognition. The major drawback of antibody use is the detection of a small molecule. As the antibody is a large molecule and has a rigid structure, no structural changes occur, and not enough specific interactions could be created with the antibody to sufficiently stabilize the binding complex (in contrast to the large moleculeantibody complexes). ${ }^{15,17}$ The use of aptamers for small molecule detection is worthy of further investigation. The aptamer is a small molecule when compared to the antibody and due to the single-stranded nature, a strong structural rearrangement of the aptamer may be produced upon the target interaction. Even when targeting large molecules, it could be more effective to use an aptamer than an antibody.

To build a biosensor platform, the rearrangement of the biorecognition elements structure is a major feature to create a sensitive and reliable biosensing signal. If the specific interaction 
creates a strong biological signal, the transduction into a significant readable physical signal would be easier, and the distinction between non-specific and specific signals will be much clearer.

The stability of aptamers allows them to be used in different conditions (high or low salinity, high or low temperature, high or low $\mathrm{pH}$ ) without being irreversibly denatured. By contrast, the changes in conditions can result in an irreversible denaturation of the antibody. ${ }^{15}, 17$ However, for aptamers, some caution is advised. They are not irreversibly denatured, but aptamers undergo structural change upon different conditions if it is outside the conditions in which the aptamer was selected (such as temperature change, salinity change, and $\mathrm{pH}$ ). Therefore, the application conditions for the aptamer must be chosen carefully and utilised during the systematic evolution of ligands by exponential enrichment process (called the SELEX process) to ensure the minimal negative impact on the performance of the aptamers.

For therapeutic purposes, aptamers are becoming a biotechnology reagent of interest to replace antibodies. Due to their biological origins and size antibodies could create a strong immunogenic response in the body. Aptamers, on the contrary, being synthetic molecules are inclined to be more discrete in the body system without causing a strong immunogenicity response. Thus, due to their size and their specificity, aptamers are being implemented in therapeutics to bypass the immunogenicity drawbacks associated with antibodies. ${ }^{15,17}$

Each transduction system used has various advantages and limitations. Selected transduction methods that integrate aptamers will be reviewed in Section 1.1.3. The choice of the most suitable transduction system is dependent on; the target molecule to recognize, the quantitative or qualitative data output required, and the cost-effectiveness and portability of the system.

\subsubsection{Aptamers}

Aptamers are synthetically designed single-stranded (ss) DNA/RNA-based oligonucleotides (oligos) that are evolved using directed evolution methodology to attain high levels of affinity and specificity to a wide variety of targets ranging from cells, proteins, peptides, small

molecules to ions. ${ }^{18-20}$ In this dissertation, ssDNA aptamers are utilised as the bioreceptor to investigate the electrochemical biosensor platform. 


\section{A general overview of deoxyribonucleic acid (DNA)}

In 1868 Miescher first isolated cell nuclei from pus cells and identified the presence of a phosphorus-containing compound. ${ }^{21,22}$ The resulting nucleic acids were found upon hydrolysis to yield the purine bases: adenine $\mathrm{A}$ and guanine $\mathrm{G}$ and the pyrimidine bases: thymine $\mathrm{T}$ and cytosine C (Figure 1.2A). Watson and Crick in 1953, ${ }^{23}$ proposed a double-stranded DNA structure (Figure 1.2B), indicating that the DNA molecule is composed of two polynucleotide chains that coil around each other to form a double helix carrying genetic instructions for the development, functioning, growth, and reproduction of all known organisms. The two DNA strands are identified as polynucleotides as they are composed of simpler monomeric units called nucleotides. Each nucleotide is composed of one of four nitrogen-containing nucleobases (cytosine $\mathrm{C}$, guanine $\mathrm{G}$, adenine $\mathrm{A}$ or thymine $\mathrm{T}$ ), a sugar subunit known as deoxyribose, and a negatively charged phosphate group. The nucleotides are joined to one another by covalent bonds (photo-diester linkage) between the sugar of one nucleotide and the phosphate of the next, resulting in a sugar-phosphate backbone. ${ }^{24,25}$ The nitrogenous bases of the two separate complementing polynucleotide strands are hybridised together, according to Watson Crick base pairing rules (A with $\mathrm{T}, \mathrm{G}$ with $\mathrm{C}$ ), with hydrogen bonds to form doublestranded DNA (Figure 1.2). 
Structure stability of the double-stranded DNA is related to different non-covalent interactions: hydrogen bonds between nucleotides to form base pairs, $\pi$-stacking interactions between each base pair, and hydrophobic interactions between the nitrogenous bases and the surrounding sheath of water (Figure 1.2B). DNA exists in biological systems mainly in the so-called Bform duplex structure which is a right-handed helical structure where the base pairs are perpendicular to the helix axis. At $\mathrm{pH}$ neutral, the whole structure is anionic due to the negative phosphate group of the DNA backbone. ${ }^{26,27}$

(A)

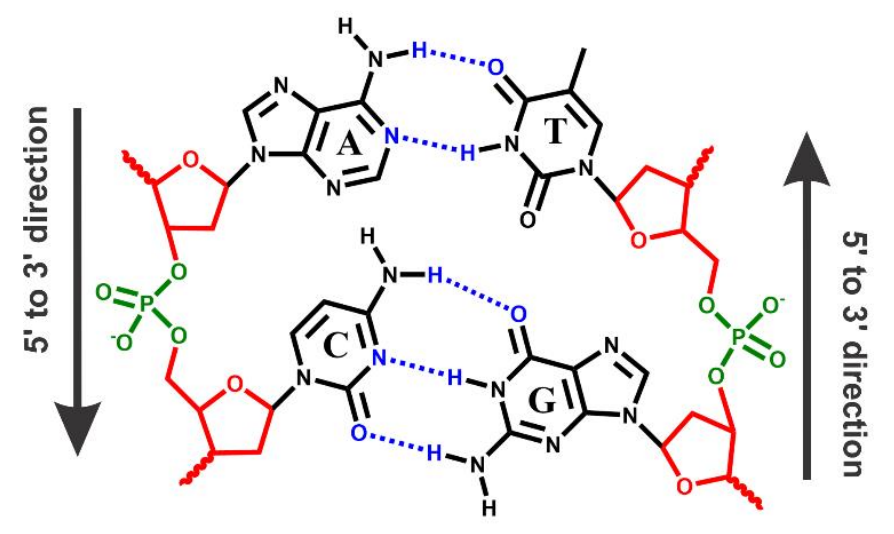

(B)

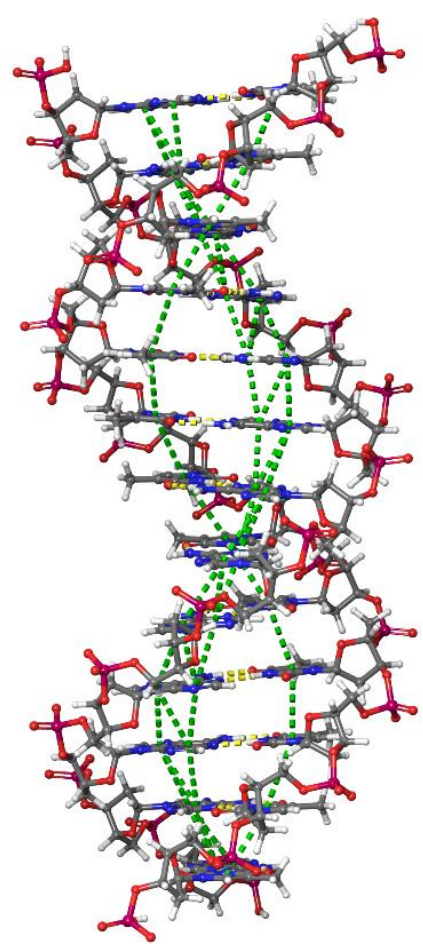

Figure 1.2. Representation of a DNA strand in two different dimensions. (A) Planar (or 2D) representation of the groups composing a DNA strand. The phosphate group (green), the sugar moiety (red), the purine and pyrimidine bases (black), and the hydrogen-bonding interactions (blue). (B) 3D representation of a double-stranded DNA with hydrogen bonding (yellow dot) and $\pi$ - $\pi$ (green dot) interactions.

\section{Production of aptamers}

Unlike the double-stranded DNA found in nature, the synthetic ssDNA aptamers are singlestranded between 20-120 nucleotide bases long. When evolved to specifically bind a target molecule of interest, aptamers attain partial double-stranded structures when associating with 
intra complementing bases and association with the target molecule. The final conformation adopted by the aptamer is highly specific for the target.

The process of selecting an aptamer for a target molecule is known as the systematic evolution of ligands by exponential enrichment (SELEX). SELEX was developed by Tuerk and Gold, and Ellington and Szostak in 1990. ${ }^{18,19}$ Aptamers are selected using in vitro methodology however, recent advances in the field allow for selection to be carried out within cultured cells. In the last decade, different SELEX protocols have been developed and optimized to generate aptamers with high affinity and specificity for the target. ${ }^{28}$ Methods of aptamer selection include; Affinity Matrix SELEX, ${ }^{28}$ Capillary electrophoresis-SELEX, ${ }^{29}$ Capture-SELEX, ${ }^{28,} 30$ Graphene oxide-SELEX, ${ }^{31}$ Gold nanoparticle-SELEX. ${ }^{32}$ However, the general main steps of aptamer selection remain the same.

Firstly, a large single-stranded DNA (ssDNA) library $\left(\approx 10^{15}\right.$ ssDNA) which consists of 3 sections (one core random region flanked by two known primer regions) is designed and chemically synthesised. The ssDNA contains a core random region where the nucleotide sequence is entirely random to generate diversity in the library. The core region is flanked by two primer binding regions with known nucleotide sequences necessary for polymerase chain reaction (PCR) amplification steps (Figure 1.3-Step 1). The library is co-incubated with the target of interest (the method of incubation of the target with the ssDNA library differs from one SELEX method to one other) (Figure 1.3-Step 2).

Following the co-incubation of the library with the target, the portion of the library that has an association with the target is separated from the portion that does not have any association. This step is commonly known as partitioning or elution of target bound library. (Figure 1.3Step 3). During the partitioning or elution, strategies are introduced to create selection pressures, resulting in the enrichment of oligo population having a higher affinity for the target within the library. The resulting target bound ssDNAs are amplified via PCR being purified (Figure 1.3-Step 4 and 5). During the PCR amplification step, strategies such as enrichment PCR or mutagenesis PCR are used to manipulate the library. Mutagenesis PCR is used to introduce variants within the selection library to potentially improve the library's affinity towards the target. The resulting purified ssDNA library is used as the template for the subsequent round of aptamer selection. Iterative rounds of selection and enrichment are carried out until sufficient target binding capability is achieved (Figure 1.3). The library is sequenced 
to identify the individual nucleotide sequence before undertaking target binding characterisation of the individual aptamers.

Additional steps are included during the SELEX process to achieve higher specificity to the target. Counter selection is a critical step to obtain higher specificity. ${ }^{33}$ Counter selection is when an interferent molecule (structurally close to the study's target of interest) is incubated with the selection library that demonstrated affinity to the target. This incubation step is included to remove oligos from the selection pool that may also have an affinity or some level of association to molecules that may interfere with the final use of the aptamer.

Usually, to undertake SELEX, a specific buffered environment is chosen that resembles the desired condition in which the aptamer will be utilised. Some aptamers have been reportedly generated in conditions such as environmental water samples, saliva, and plasma. ${ }^{34}$ The condition is used to enhance the production of enthalpically driven aptamer-ligand interaction (specific bonds creation such as hydrogen bonds, Van der Walls interactions) in the final application. ${ }^{35}$ 
Mostly, no target binding information is collected during the SELEX process. Aptamers target binding affinity and specificity are usually determined at the end of the selection process. Methods such as surface plasmon resonance (SPR), ${ }^{36}$ fluorescent affinity assays, ${ }^{36}$ isothermal titration calorimetry (ITC), ${ }^{34}$ microscale thermophoresis (MST) ${ }^{37}$ are widely utilised.

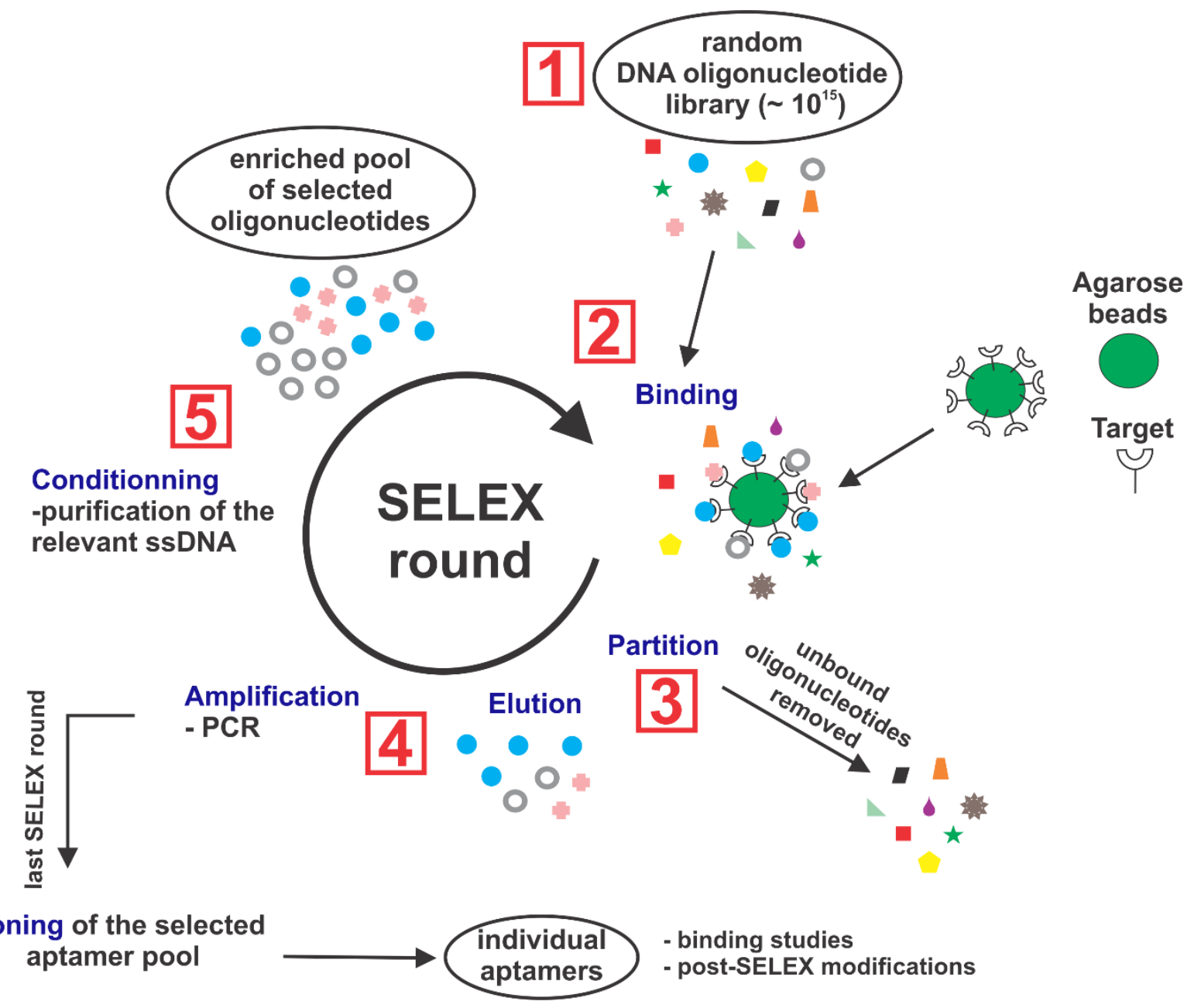

Figure 1.3. Schematic illustration of a SELEX process where the target is fixed on agarose beads. The process has been separated in five different steps.

Additionally, to the binding affinity determination, it is observed that the collection of thermodynamics and structural information is required especially for characterising small molecule-aptamer binding. A structural observation technique could be the nuclear magnetic resonance spectroscopy (NMR). NMR is used to obtain a ${ }^{1} \mathrm{H}-\mathrm{NMR}$ spectrum in the special resonance region of the DNA strand imino protons. ${ }^{38}$ The appearance, shifting, or disappearing of peaks in this resonance region is crucial information that could reveal some compelling binding event knowledge. It is through these protons that most of the specific interactions 
occur. Formation of base pairing in the aptamer structure (Watson-Crick or non-Watson-Crick) or formation of hydrogen bonding between nucleotides and the target of interest could be observed.

In addition to the binding affinity determination, ITC could also be used for the acquisition of thermodynamic information. Indeed, the thermodynamic of a binding reaction can be separated into two main groups: entropically and enthalpically driven binding reaction. ${ }^{35}$ The entropically driven binding reaction is related essentially to the release of the water molecule attached to the aptamer or the ligand, in contrast to the enthalpically driven binding reaction that is related to the formation of specific bound between the aptamer and the ligand such as hydrogen bonds and van der Waals interactions. ${ }^{39}$ Consequently, the aptamer-ligand enthalpically driven reaction is favored for the aptamer selection process.

\section{Interactions between aptamers and their target}

Aptamers possess various folding topographies composed of different elements, such as loops, hairpins, stems, and quadruplexes. ${ }^{40}$ Each nucleotide in an aptamer sequence either has direct contact with the target molecule as a binding site or with another nucleotide within the sequence to sustain the overall structure (Figure 1.4). ${ }^{13}$ In an aptamers native state, aptamers fold and associate with nucleotide bases within themselves, aptamers are partially double-stranded DNA, their partial duplex structures can possess different forms: B-form, A-form, Z-form, or G-quadruplex form. ${ }^{41}$ However, when interacting with the target, the recognition event could involve a change in the duplex structure (Figure 1.4). Nucleotides are nitrogen-rich bases and have different chemical functional groups that can interact with other molecules through hydrogen bonds or/and Van der Walls interactions. The negative phosphate groups belonging to the phosphate backbone of the DNA strand are also inclined to electrostatically interact with cationic species or between each other for repulsion and stability purposes.

The major parameter used in literature to define the strength of binding between an aptamer and its specific ligand is the dissociation constant $\left(K_{D}\right) \cdot{ }^{40} \mathrm{It}$ is a thermodynamic parameter that is related to the affinity of the receptor with the ligand. A high-affinity $K_{D}\left(K_{D} \approx\right.$ low nanomolar range) refers to a very strong binding with a large negative free Gibbs energy $\left(\Delta G^{0}\right)$. The dissociation constant is the inverse of the association constant $K_{a}$ and can be calculated with the following equation in the simplest case of a 1:1 stoichiometry: 


$$
A+B \leftrightarrow A B \text { where } K_{D}=\frac{[A] \times[B]}{[A B]}
$$

The dissociation constant parameter is important for the design of a detection platform. Indeed, the "active" working range can be deduced from it. As described in Wilson et al., ${ }^{42}$ the useful detection range of an affinity reagent spans in target concentration centered around the $K_{D}$. With this $K_{D}$ value, it is possible to use the specific ligand concentration range for the development of the platform to avoid nonspecific interactions of the ligand with other surfaces or molecules rather than the DNA strand. However, the determination of the dissociation constant must be done with caution. As explained by McKeague et al., ${ }^{36}$ aptamer binding varies when either the target or aptamer is used in solution vs immobilized, and the sensitivity of each technique affects the apparent affinity. It has been concluded that more than one binding affinity assay must be used to have a reliable $K_{D}$ value. However, to obtain the most reliable $K_{D}$ value, a technique such as the isothermal titration calorimetry (ITC) must be used because the aptamer and the target are free in solution and, therefore, it will reveal the more "natural" binding mechanism related to the aptamer-target complex.

(A)

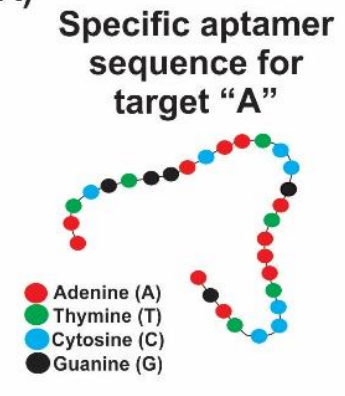

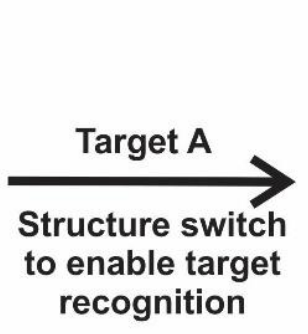

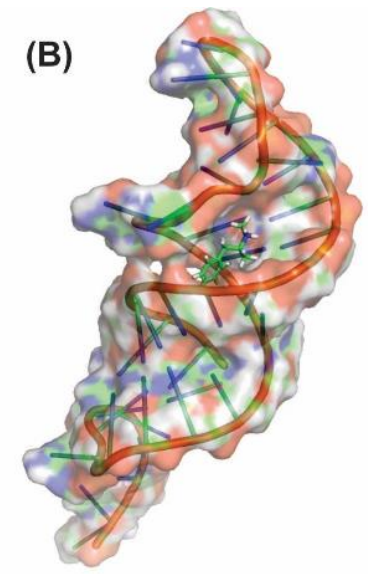

Figure 1.4. Representation of the binding event in two different dimensions. (A) The incubation of target $\mathrm{A}$ induces a restructuration of the DNA strand to form a specific binding pocket for A. New hydrogen bonds are formed intra-DNA strand and between the analyte A and some nucleotides. (B) 3D representation of a DNA strand where a specific binding pocket is formed for the analyte (green molecule).

\section{Thermodynamic view of the binding event}

The thermodynamics parameters obtained via ITC experiments are valuable. As mentioned previously, both the receptor and the ligand are free in solution. ${ }^{43}$ ITC is a label-free direct 
measurement of the heat evolved or absorbed during a binding reaction. ${ }^{43,44}$ It can be concluded that the $K_{D}$ calculated with ITC is only dependent on the sensitivity of the ITC instrument used. The association constant $K_{a}$, at reaction equilibrium, could be related to the binding free energy change $\Delta G$ with the equation (1.2): $:^{35,39,43}$

$$
\Delta G=-R T \ln K_{a}
$$

where $\Delta G$ is the Gibbs free energy change, $K_{a}$ is the association constant related to the binding reaction, $R$ is the universal gas constant, and $T$ is the temperature.

The main thermodynamic parameters are involved in the Gibbs free energy equation ${ }^{35,39,43}$

$$
\Delta G=\Delta H-T \Delta S
$$

where the Gibbs free energy change $(\Delta G)$ represents the thermodynamic potential of a system to produce maximum or reversible work at a constant temperature $T$, and pressure $P$. In analogy with a binding process investigation, the binding can only occur if the change in Gibbs free energy is negative. ${ }^{35}$ The enthalpy change $(\Delta H)$ reflects the gain (endothermic) or the loss (exothermic) of heat during the binding event. The creation of specific binding such as hydrogen bonds and Van der Walls interactions produces heat, while disruption of these interactions, on the contrary, induces absorption of heat during the binding process. ${ }^{35}$ The entropy $(\Delta S)$ is the measure of how evenly the heat energy will be distributed over the overall thermodynamic system. It could also be a measure of the number of microstates available to the system. ${ }^{35}$ The term could be separated into three terms:

$$
\Delta S=\Delta S_{\text {solv }}+\Delta S_{\text {conf }}+\Delta S_{r / t}
$$

where $\Delta S_{\text {solv }}$ represents the solvent interaction from surface burial upon binding. The solvent is released which results in a favorable contribution to the overall entropic term. The water entropy is a key quantity in elucidating the folding/unfolding mechanisms of biomolecules. ${ }^{45}$ $\Delta S_{\text {conf }}$ reflects the change in conformation freedom of both the aptamer and ligand upon binding. The contribution could be favorable or unfavorable following the degree of freedom difference between the complex formed and the two molecules free in solution. ${ }^{35} \Delta S_{r / t}$ represents the loss of translational and rotational degrees of the freedom of the aptamer and 
ligand upon complex formation. Its contribution is unfavorable due to the reduction of the number of free particles in solutions. ${ }^{35}$

The aptamer-ligand binding is driving through the decrease in system free energy. The two quantities $\Delta H$ and $\Delta S$ determine the sign and magnitude of the free energy change, and they are closely related. When a tight binding occurs with many non-covalent interactions created, $\Delta H$ will be negative; however, it will be combined with an unfavorable entropy compensation due to the restriction of the mobility of the interacting partners. ${ }^{35,46}$ On the contrary, a binding event where the $\Delta S$ is positive (so favorable) is often accompanied by a penalty $\Delta H$ because of the energy needed to disrupt the solvent molecule non-covalently interacting with the receptor or the ligand. These thermodynamic parameters can be related to the general binding mechanism of the receptor and the ligand. The next section introduces three major binding mechanisms. 


\section{Three main binding mechanisms: lock and key, conformational selection, and induced fit}

The same general binding mechanisms associated with protein-ligand binding can be used to study the aptamer-molecule biorecognition events. As shown in Figure 1.5, three different binding mechanisms exist: lock and key ${ }^{47}$, conformational selection ${ }^{48,49}$, and the induced fit ${ }^{50}$ binding mechanism.

(A) Lock-And-Key
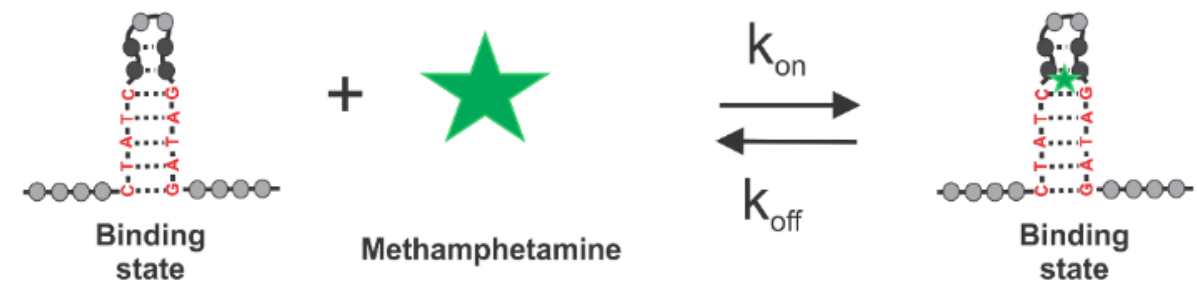

(B) Conformational-Selection
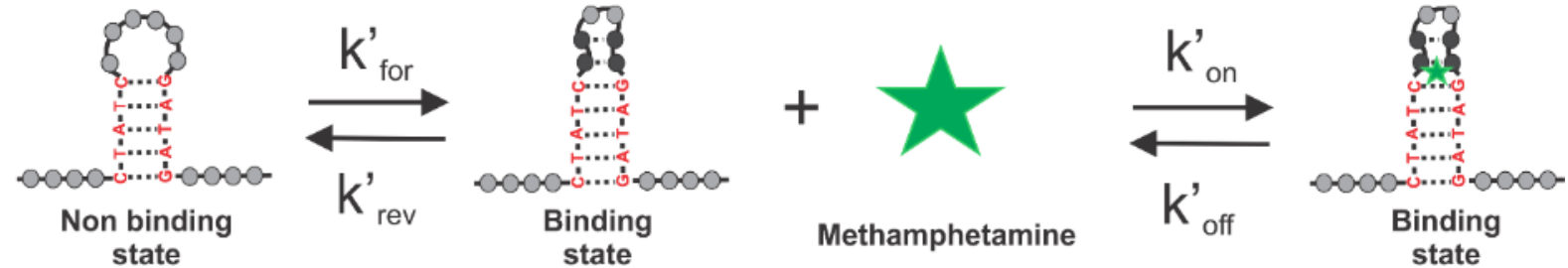

(C) Induced-Fit
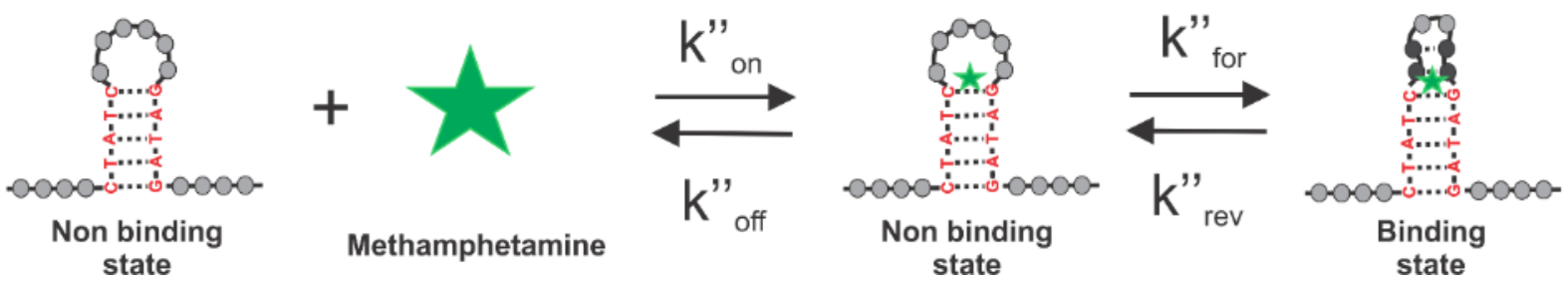

Figure 1.5. Schematic illustration of three binding mechanism related to the aptamer-methamphetamine interaction. (A) Lock-and-key, (B) Conformational-selection, and (C) Induced-fit binding models.

The "lock-and-key" (LAK) binding model representation is the most common in enzymology or protein-ligand interactions (Figure 1.5A). It can be explained as an interaction of an antibody with its specific ligand. No aptamer structural changes are related to the target interaction; the aptamer retains the same structure before and after the interaction. It is a strong entropically driven reaction for an aptamer-small molecule interaction. ${ }^{35,51,52}$ The small molecule has a few functional groups that can interact via hydrogen bonding with the aptamer. The enthalpy contribution is either negatively or positively small. As no movement of the aptamer is produced, the only movement is created by the surrounding water molecules and 
counterions disruption with the ligand addition. Indeed, the system becomes disordered and a large water gain entropy is monitored. The ligand-aptamer hydrophobic effect can be explained via this water entropy gain. ${ }^{35}$ When the ligand interacts with the specific binding pocket of the aptamer, it replaces some water molecules. The binding constant equation related to the "lockand-key" equilibrium is given as

$$
K_{D}^{o b s}=\frac{k_{o f f}}{k_{o n}}
$$

The "conformational selection" (CS) binding mechanism starts with an equilibrium between different forms of the receptor in solution (Figure 1.5B). ${ }^{53-55}$ In this case, the aptamers are flexible and can produce different forms in solution. It is unexpected to observe only one aptamer structure in solution especially at mild conditions (room temperature and normal salinity). The equilibrium between the two forms is disrupted upon the target addition in the system. Only one of the two (or more) forms is specific to the target and can create a specific biorecognition event. Some studies have been shown to create a stronger response upon target addition by modulating the equilibrium between the two unbound aptamer forms. ${ }^{53}$ From a thermodynamics point of view, it is an entropically driven reaction. ${ }^{56}$ The binding constant equations related to the "Conformational Selection" equilibrium are the following:

$$
K_{D}^{o b s}=K_{\text {disso }}\left(1+K_{\text {iso }}\right) \text { where } K_{\text {disso }}=\frac{k^{\prime}{ }_{o f f}}{k^{\prime} \text { on }} \text { and } K_{\text {iso }}=\frac{k^{\prime} \text { rev }}{k^{\prime} \text { for }} \text {. }
$$

Lastly, the "induced fit" (IF) binding mechanism is a compelling binding mechanism. The target induces a new form of the receptor (Figure 1.5C).$^{51}$ Before the ligand addition, one form of the receptor is in solution. When the target is added, it induces a structural change of the aptamer. On the contrary to the two previous forms explained, this binding mechanism is enthalpically driven. ${ }^{35}$ Some specific interactions, such as intra-hydrogen bonding and Van der Waals interactions, are created to modify the structure of the aptamer. Thus, a strong negative enthalpy is generated. Indeed, in the two previous forms, the creation of an $\mathrm{H}$-bond between the target and the aptamer is most of the time not strong enough to create an enthalpy signal that can compensate for the large water entropy gain. In the induced fit case, it is possible. The binding constant equations related to the "Induced Fit" equilibrium are the following:

$$
K_{D}^{o b s}=K_{\text {disso }} \frac{K_{\text {iso }}}{\left(1+K_{\text {iso }}\right)} \text { where } K_{\text {disso }}=\frac{k^{\prime \prime}{ }_{\text {off }}}{k^{\prime \prime} \text { on }} \text { and } K_{\text {iso }}=\frac{k^{\prime \prime} \text { rev }}{k \prime \prime \text { for }}
$$




\subsubsection{Aptamer-based biosensors: aptasensor technology}

When an aptamer is combined with a physical transduction system to detect a target of interest, an aptasensor platform is created. In the previous sections, we discussed the aptamers and their mechanism of recognizing a molecule. Numerous transduction systems have been developed based on different physical ways of reading the specific signal. Historically, in the aptasensor technology, the first transduction system has been a flow cytometry system in $1996 .{ }^{57}$ This system has been developed for human neutrophil elastase detection.

This section will briefly describe some of the main transduction systems used for molecule detections using an aptamer as a biorecognition element. As described in Figure 1.1, some techniques have been used to transduce the biorecognition event. The platform used for the signal transduction must be chosen carefully following the nature of the ligand and the requirement for the level of detection and quantitation.First, optical aptasensors can be classified into two different classes: colorimetric and fluorescent aptasensors. Both are based on a spectral light that is monitored along with the sensing experiment. ${ }^{58}$

\section{Colorimetric optical aptasensor}

A common colorimetric aptasensor uses gold nanoparticles (AuNPs). The gold nanoparticle (AuNP) aptasensor assay is related to the ability of the AuNP to have a plasmonic response to the light. The visual colour change is a result of the surface characteristics of the AuNP. The fixation of the aptamers on AuNP is dependent on the affinity and capacity of adsorption of nitrogen bases of the aptamers on the gold surface. ${ }^{59}$ In a mild ionic strength condition, the AuNPs do not aggregate. The AuNPs colour is red (naked-eye visible). When the salt concentration of the solution is increased, the possibility of the AuNPs to aggregate is increased too. When AuNPs aggregate, the colour solution turns blue.

However, as the aptamers are non-covalently adsorbed on the AuNPs surface they avoid the AuNPs aggregation. The addition of the ligand (specific to the aptamers in solution) creates a competition of affinity between the aptamer-AuNP interaction and the aptamer-ligand interaction. As the affinity between the aptamer and the ligand is higher in comparison with the non-specific interaction between AuNP and aptamer, the aptamers dissociate from the AuNPs surface to interact with its specific target. Therefore, AuNPs are no longer protected from the ionic environment. An AuNPs aggregation effect can be monitored proportional to 
the target concentration used. The aggregation of the AuNPs produces a purple/blue colour that is visible to the naked eye (Figure 1.6A). Alsager et al. ${ }^{60}$ reported a colorimetric aptasensor strategy for $17 \beta$-Estradiol detection. By fixing non-covalently the aptamer strands on the AuNPs and doing some truncations studies on the aptamer, they have been able to reach a limit of detection of 200pM. This system is compelling for its low cost and simplicity of use (Figure 1.6A).

However, the sensing scheme has some major drawbacks that could be a big concern for the reliability of the aptasensor results. As Zong et al. ${ }^{61}$ explained, the AuNPs surface is a reactive surface that can easily react nonspecifically with different molecules. For instance, the AuNPs aptasensor assay for arsenite has a major nonspecific signal issue. ${ }^{61}$ By reacting with the AuNPs, the arsenite enables an aggregation effect not produced by the specific interaction between the aptamer and the arsenite but rather the arsenite and the AuNPs. Thus, the signal change observed could be mainly due to the nonspecific interaction and not to the specific binding signal. Only one signal can be monitored in AuNPs experiments (absorbance of AuNPs in solution), therefore, it is difficult to enable a clear separation in the readable signal between a specific and a non-specific interaction. To conclude, the robustness of this sensing scheme can only be validated after undertaking extensive control experiments which include testing the interaction between bare AuNPs and the target of interest. ${ }^{62}$ In addition, a control experiment that uses a randomized aptamer sequence is not trustable enough due to the difference in adsorption of each aptamer on AuNPs because of the difference in each nucleotide sequence. 
Another technique of fixation of the aptamer on AuNPs could be used for better control of the system. As described in Liu et al., ${ }^{63}$ the aptamer is covalently attached to the gold nanoparticle via a strong sulfur-gold bond. This assay is based on the dispersion of aggregated gold nanoparticles upon the ligand addition (Figure 1.6B). On the contrary to the AuNPs assay mentioned above, the colour solution will change from purple to red. Through this specific sulfur-gold bond, better control experiments could be designed. A randomized aptamer sequence could be attached similarly to the aptamer sequence on gold nanoparticles. For removing entirely the non-specific adsorption of nucleotides on the gold surface a blocking agent, such as the 6-mercapto-1-hexanol (MCH), can be used. ${ }^{64}$

(A)
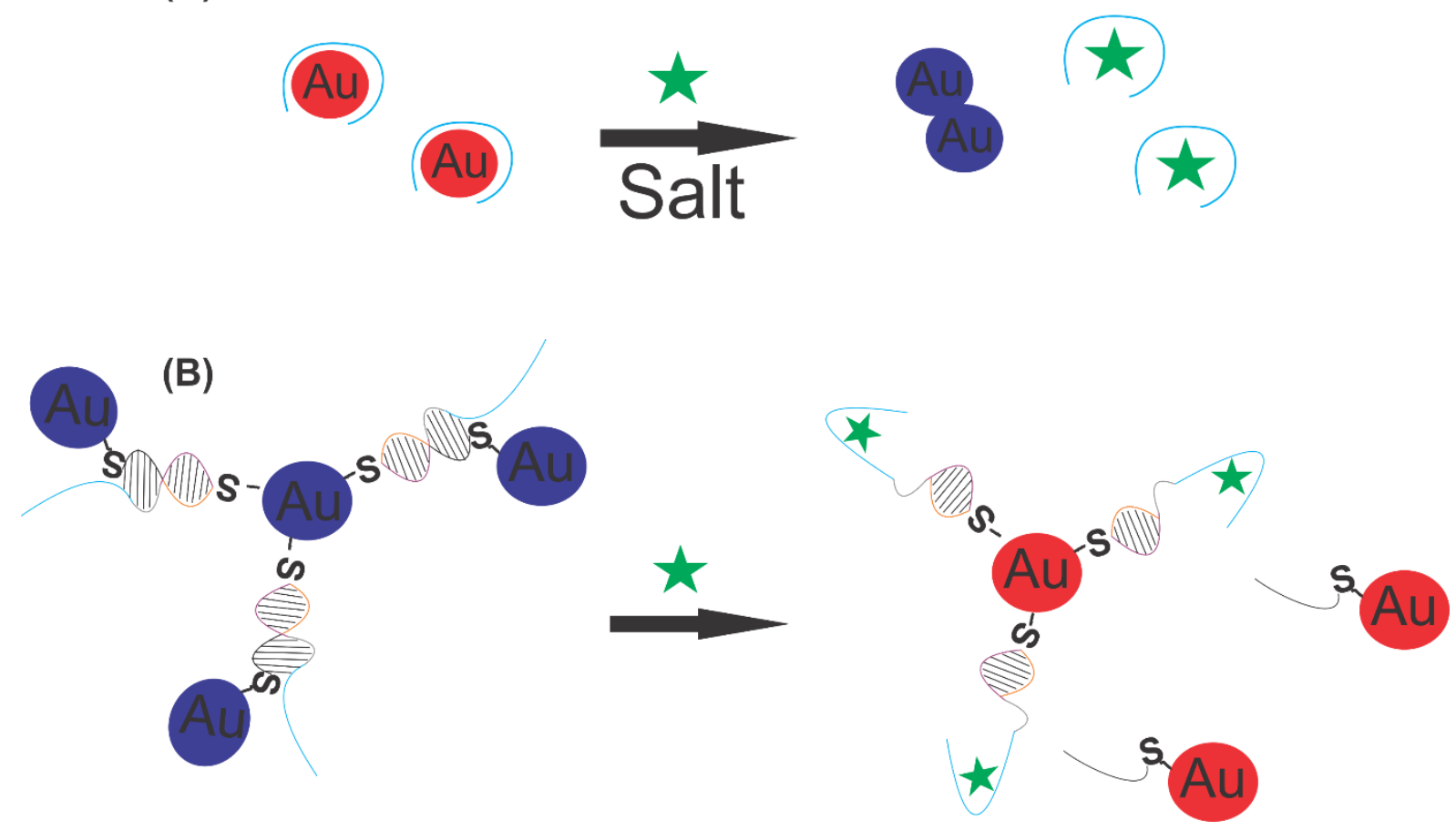

Figure 1.6. Schematic illustration of two sensing strategies employing aptamers and optical sensing based on AuNPs. (A) By interacting with the aptamer, target induces aptamer to dissociate from the AuNPs and therefore, induces the AuNPs to aggregate at a specific salt concentration. (B) An Aptamer (blue line) used to hybridise and cross-link two complementary sequences (orange and black lines) coupled to AuNPs. Recognition of the target releases the complementary sequence adjacent to the binding cavity and breaks down the aggregates.

\section{Fluorescent optical aptasensor}

Optical detection by fluorescence is one of the most popular signal transduction methods due to the availability of many fluorophores and quenchers. ${ }^{65}$ Recognition events are translated 
based on quenching or enhancement of the fluorescent light initially present in the system. The fluorescent probe is covalently attached to the aptamer. ${ }^{65}$

Stonajovic et al. ${ }^{66}$ demonstrated a selective aptasensor system for the detection of cocaine in the micromolar range. The cocaine aptamer was labelled with a fluorophore and a quencher at each end. In the absence of cocaine, the aptamer structure is open, and a strong fluorescent signal can be monitored. Upon cocaine addition, the fluorophore and the quencher are next to each other, thus the fluorescent signal is quenched. This fluorescent signal reduction is the specific binding signal that enables sensitive and reliable detection of cocaine (Figure 1.7B).

In Munzar et al. ${ }^{67}$ a duplexed aptamer is used. The duplex aptamer is formed with an aptamer and a complementary sequence. At the end of the aptamer, a fluorophore is covalently attached. When the duplex is formed, the complementary sequence has a fluorophore quencher covalently attached at its end. The quencher and the fluorescent probe are close when the duplex is formed, thus no fluorescent signal is monitored. Upon target addition, the structure switch of the aptamer enables a dissociation between the aptamer and its complementary sequence and therefore, a fluorescence signal is monitored (Figure 1.7A).

(A)
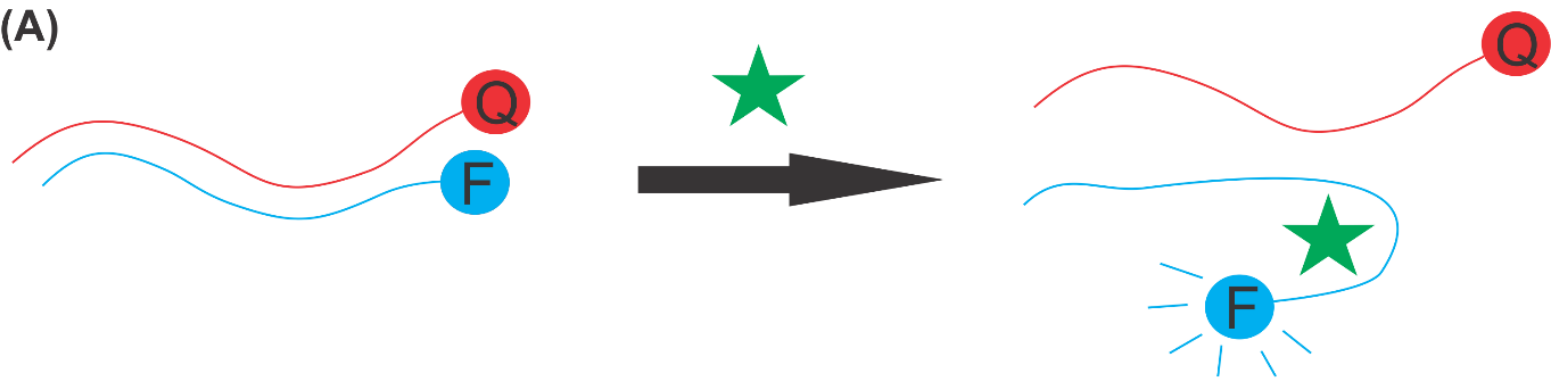

(B)
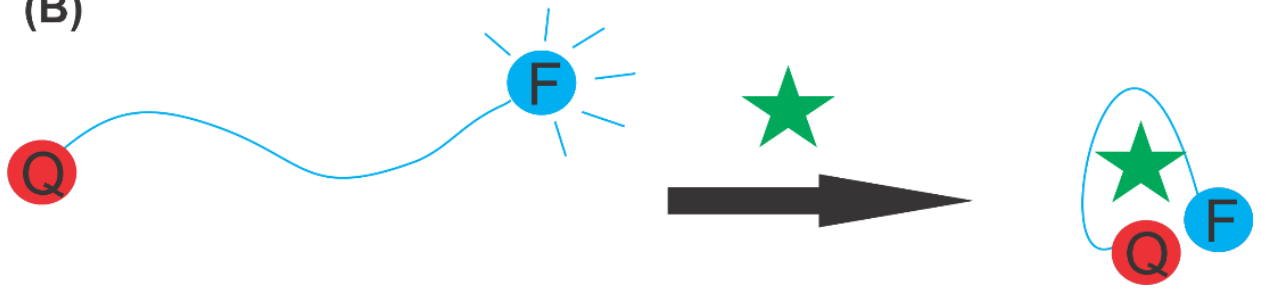

Figure 1.7. Schematic illustration of two sensing strategies employing aptamers and optical sensing based on fluorescence. (A) Aptamer (blue line) is modified with a fluorescence probe. A complementary sequence (red line) to the aptamer is modified with a quencher. A fluorescent signal is monitored after target addition which induces the dissociation of the aptamer with the complementary sequence. (B) Aptamer is modified at one end with a fluorescence probe and the other end with a quencher. The addition of the target produces a decrease of the fluorescence signal due to the proximity of the fluorescence probe and the quencher. 


\section{Electrochemical aptasensors}

One of the major and most compelling transduction systems for biosensor technology is the electrochemical platform. ${ }^{68-70}$ Electrochemical sensing usually requires a reference electrode, a counter electrode, and a working electrode, also known as the sensing electrode (Figure 1.8). The reference electrode, commonly made from $\mathrm{Ag} / \mathrm{AgCl}$, is kept at a distance from the reaction site to maintain a stable potential. The counter electrode establishes a connection to the electrolytic solution to enable a current to the working electrode, it must be conductive and chemically stable. The working electrode serves as the transduction element in the reaction. ${ }^{71}$, ${ }^{72}$ Concerning the latter, the electrode material, its surface modification, or its dimensions greatly influence its detection ability. The reaction under investigation would either generate a measurable current, a measurable potential, a measurable impedance, or a field-effect which will be measured with the appropriate method of measurement. ${ }^{71,72}$ All these points will be reviewed in the next section.

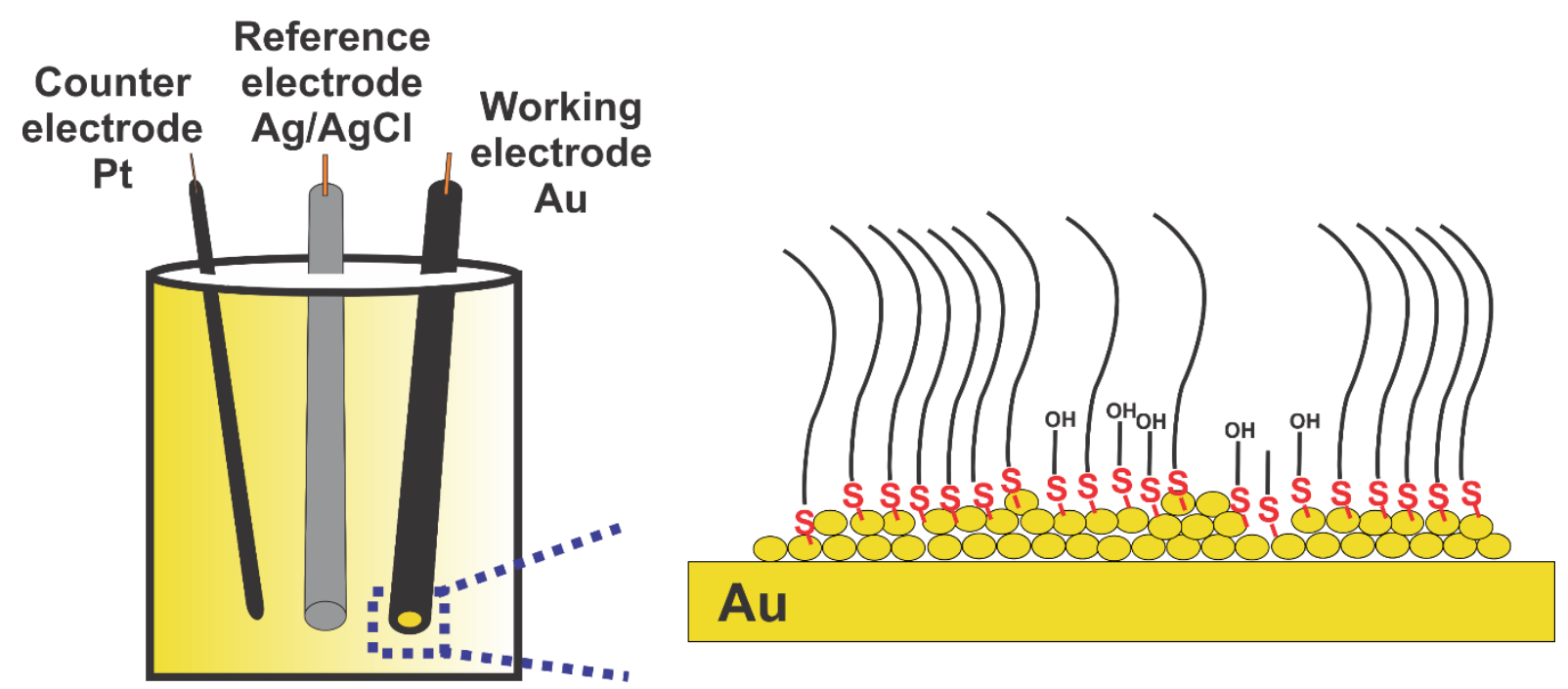

Figure 1.8. Three electrode system schematization. Here, the counter electrode is platinum, the reference is $\mathrm{Ag} / \mathrm{AgCl}$, and the working electrode is gold. Here, the gold electrode is modified with thiolated aptamers and thiolated blocking agents.

\section{Electrodes and nanomaterials modifications}

The working electrode used can be modified with different materials to enhance the detection signal or to avoid nonspecific interactions. Some nanomaterials can provide a high surface area with high conductivity and good biocompatibility. 
The carbon nanotubes are one of the most well-known and used nanomaterials. They offer unique advantages or properties, such as high surface to volume ratio, high thermal and electrical conductivity, chemical stability, and biocompatibility. ${ }^{73}$ Park et al. ${ }^{74}$ developed an electrochemical aptasensor for thrombin detection using a single-walled carbon nanotube coated on glassy carbon electrodes.

Gold nanoparticles can be deposited onto the working electrode. The deposition introduces many advantages to the biosensors, encompassing their ability to provide an efficient loading platform for immobilizing biological material and further improving the electron transfer between the active site and electrode. ${ }^{75-77}$ An impedimetric aptasensor for Ochratoxin A detection on the base of aptamer carrier based on Au nanoparticles deposited on electrode has been developed. ${ }^{78}$

Graphene is a carbon nanomaterial with outstanding physical and chemical properties. It has a large specific surface area and high electrical conductivity. In addition, it also shows great electrochemical properties, including wide electrochemical potential windows, low chargetransfer resistance, and excellent electrochemical activity. ${ }^{79}$ Kaur et al ${ }^{80}$ described an electrochemical aptasensor for Ochratoxin A detection based on graphene for increasing the electroactive surface area of the electrode.

A conducting polymer, as described in Zhu et al., ${ }^{81}$ could be a significant amplifier of the electrochemical signal. With the nanoporous morphology of the deposited conducting polymers, it has the effect of amplifying the electron transfer resistance signal to reach a femtomolar limit of detection.

\section{Methods used for aptamer fixation on gold electrode surface}

The aptamer is immobilized on the working electrode to enable reliable detection of the analyte. The most common methods used for the creation of a biological layer on an electrode will be presented in the next section. The following discussion is specifically related to a gold working electrode. 


\section{a. Thiolated aptamers}

Most of the immobilization strategies rely on the strong interaction of thiol and gold. ${ }^{82-85}$ Aptamers are covalently modified with a thiol group (-SH) either directly or via an alkyl chain. The formation of the monolayer follows a four-step mechanism ${ }^{85,86}$ :

1) Diffusion-controlled physisorption where only van der Waals interactions account for the adsorption. Nonspecifically adsorption of nitrogen atoms from ssDNA mainly occurs (Figure 1.9A). ${ }^{87}$

2) Chemisorption of the sulfur head group that bonds to the gold surface. A strong bond is formed about $50 \mathrm{kcal} / \mathrm{mol}$ (Figure 1.9B).$^{82,86,88}$ The nature and mechanism of the chemisorption is still part of a scientific controversy. ${ }^{89,90}$

3) After the chemisorption process, the growth process of the organic monolayer starts. Nucleation at some preferential sites (defective sites on the gold surface) occurs (Figure 1.9C). ${ }^{86}$

4) After nucleation, the surface coverage of thiolated species increases on the $\mathrm{Au}$ surface. Van der Waals, repellent, steric, and electrostatic forces form a highly ordered and orientate self-assembled monolayer (SAM) with standing-up conformation (Figure 1.9D). ${ }^{82,86}$ 
As shown in Figure 1.9, during step A, the possibility of strong nonspecific adsorption of ssDNA onto gold electrodes can occur.87 To form a compact and stand-up layer, a thiolated alcohol molecule could be used for this purpose as 6-mercapto-1-hexanol (MCH).84 MCH displaces the nonspecifically adsorbed parts of the aptamer and ensures their upright orientation owing to the repulsion between the net negative dipole of the $\mathrm{MCH}$ alcohol terminus and the negatively charged DNA backbones.

(A)

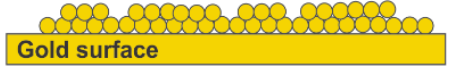

(B)

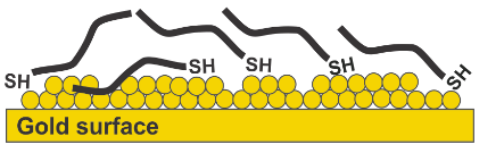

(C)

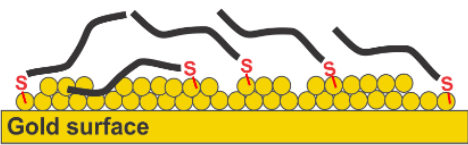

(D)

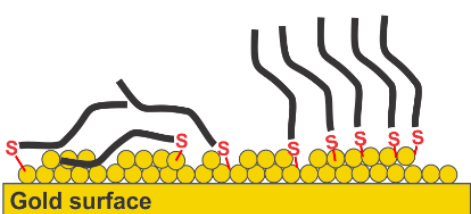

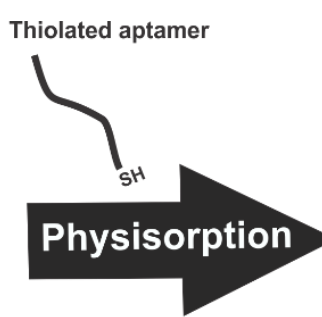
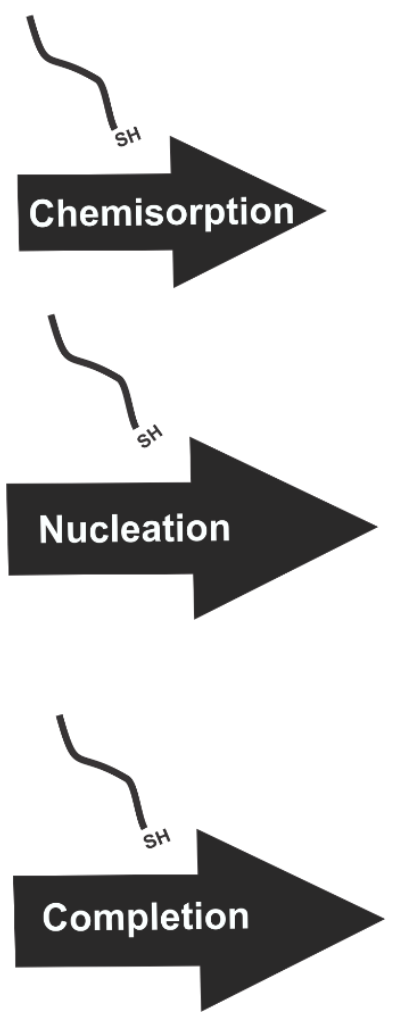
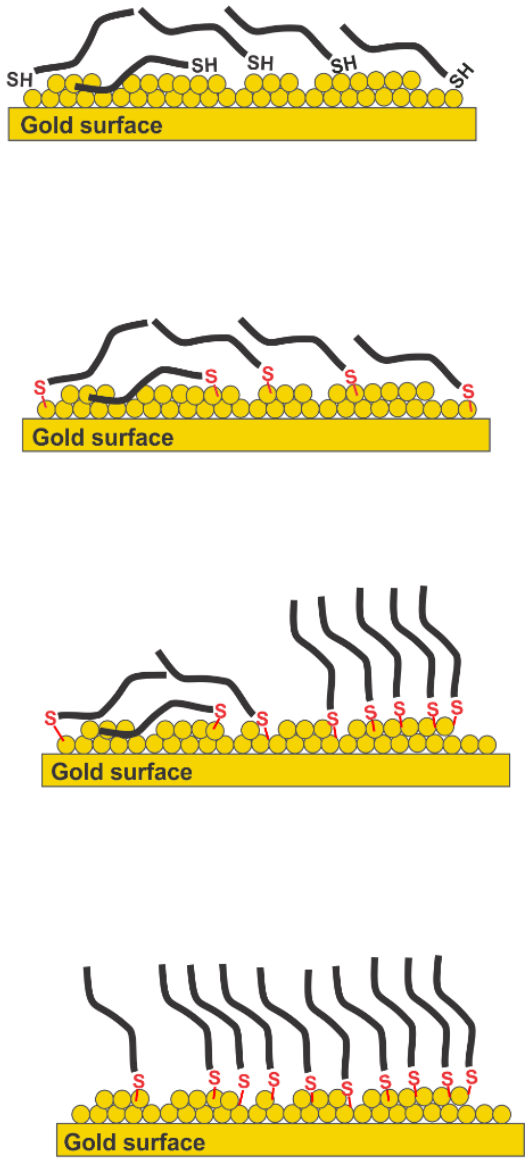

Figure 1.9. Schematic illustration of the interaction of an SH-ssDNA with a gold electrode. The interaction can be separated in four different steps. (A) Physisorption, (B) chemisorption, (C) nucleation, and (D) completion. 


\section{b. Short thiolated linkers and EDC/NHS or cysteamine/glutaraldehyde}

Short linkers can be assembled on the electrode via Au-S chemistry, to which the aptamers are afterward bound. ${ }^{91,92}$ The linkage between the aptamer and the short linker can be performed via different chemistry: the most common is the EDC/NHS chemistry. For this protocol, the aptamer is modified with a primary amine at its terminal. The reaction of carboxylic acids and primary amines is often used in aptasensor fabrication. Indeed, the amide bonds formed are highly favourable for stable immobilizations due to their poor reactivity. An aptasensor for the detection of endotoxin has been fabricated..$^{91}$ The 3-mercaptoproprionic acid was used as the short thiolated linker to whose carboxylic group the aminated aptamer was bound using EDC/NHS chemistry (Figure 1.10A).

As an alternative to the EDC/NHS coupling method, an amine coupling process using cysteamine/glutaraldehyde is employed (Figure 1.10B). Malvano et al. ${ }^{92}$ fixed an anti-gliadin aptamer via this method. The cysteamine binds to the electrode via its thiol group and builds a SAM, onto which glutaraldehyde binds to provide aldehyde groups for the subsequent binding of aminated compounds. Ethanolamine is added subsequently to passivate the remaining carbonyl groups. 
The strong affinity between streptavidin and biotin has been used for bioreceptor immobilization on electrode surfaces. ${ }^{93,94}$ Streptavidin is a large tetrameric molecule $(70 \mathrm{kDa})$ with four identical binding sites, and biotin is a small molecule that binds to these binding sites with high affinity. The interaction is nearly equal to that of a covalent bond and so, this interaction can only be broken under very extreme conditions. Zhang et al. ${ }^{95}$ immobilized biotinylated aptamer of thrombin onto a microplate modified covalently with biotin and then with streptavidin.

(A)
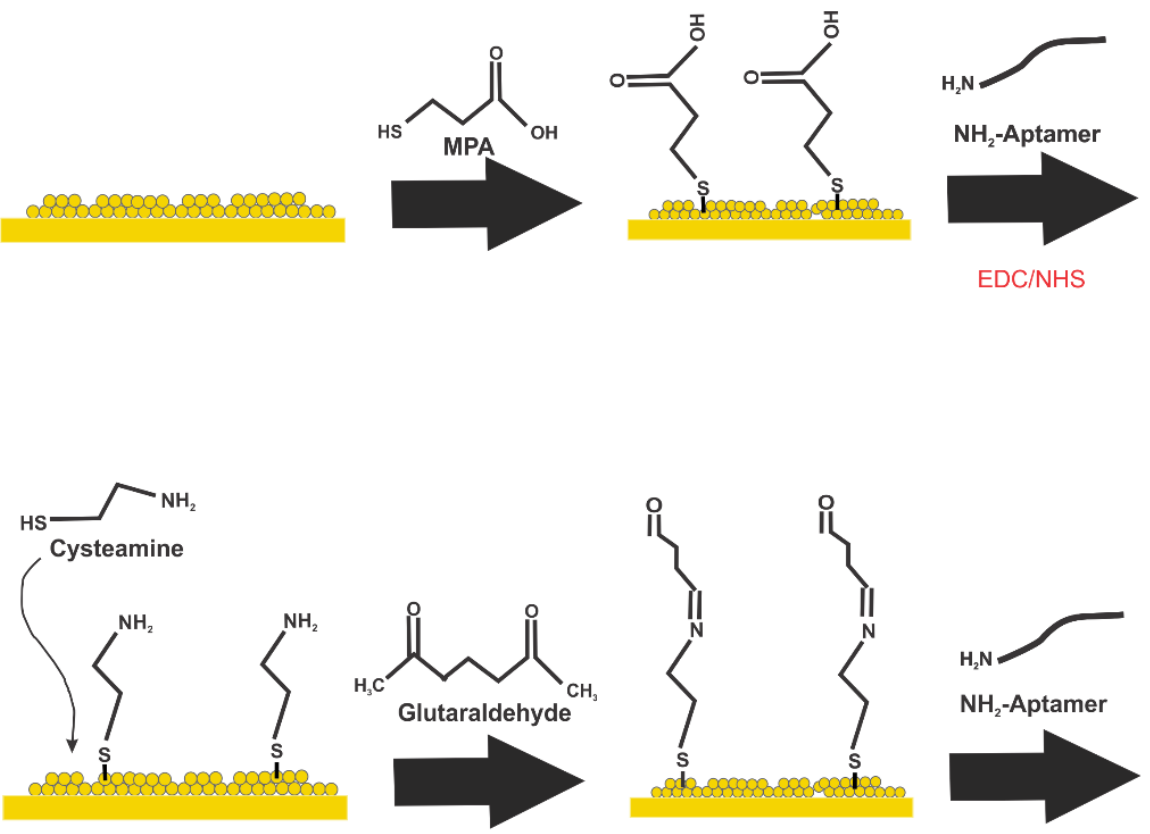

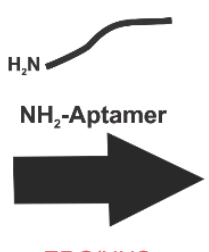

EDC/NHS

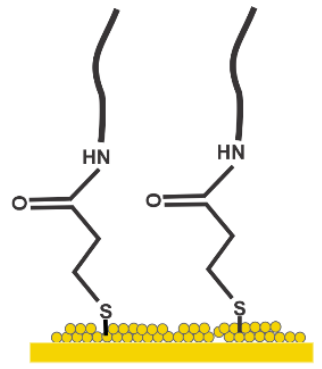

1
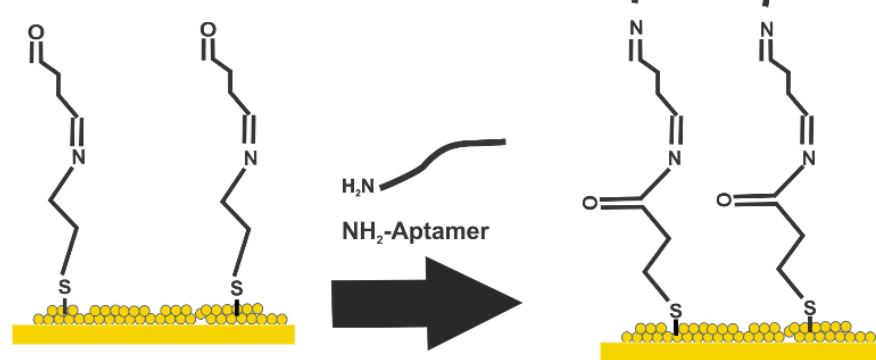

Figure 1.10. Representation of two different protocol of fixation. The ssDNA is modified with an amino group. (A) EDC/NHS fixation method and (B) cysteamine/glutaraldehyde fixation method.

\section{Different electrochemical measurement methods}

The theory related to each electrochemical method presented in the next section will be explored in Chapter 2. Here, a succinct literature review about each electrochemical method strategy is introduced in the context of aptamer-based biosensors.

\section{a. Amperometry}

Amperometric aptasensors are focused on the measurement of current as a function of time. It results from the oxidation and reduction of redox species in a biochemical reaction that depends 
on the concentration of an analyte with a fixed potential. ${ }^{96}$ When the current is measured at a constant potential, this is referred to as amperometry. However, when the current is measured during controlled variations of the potential, this is referred to as voltammetry. Huan et al. ${ }^{97}$ used an amperometric aptasensor for sulfadimethoxine detection. The electroactive toluidine blue has been used as the electron transfer producer. The response generated from this electron transfer was quantitatively related to sulfadimethoxine.

For an electrochemical aptamer-based biosensor, the voltammetry method is mainly used. The current is measured with the use of a redox probe: Methylene Blue (MB). MB is attached covalently to a part of the ssDNA or interacts non-covalently by intercalation into the ssDNA. A well-established method is described by Plaxco and coworkers for the thrombin or the cocaine detection. ${ }^{98,99}$ The MB probe is attached covalently to the 3 'end and a thiol group is attached to the 5'end of the specific aptamer. A self-assembled monolayer (SAM) is formed on the gold electrode with the use of a blocking agent $\mathrm{MCH}$. The current related to the redox reaction of the methylene blue is monitored by the controlled variations of the potential. The current measured is dependent on the proximity of the redox probe to the gold electrode surface. Thus, when the aptamer is binding, a structure switch is induced, and therefore, a change in the current intensity is measured. A signal-on system is used for cocaine detection (Figure 1.11A) $;{ }^{99}$ while a signal-off system is monitored for the thrombin detection (Figure 1.11B). ${ }^{98}$

(A)
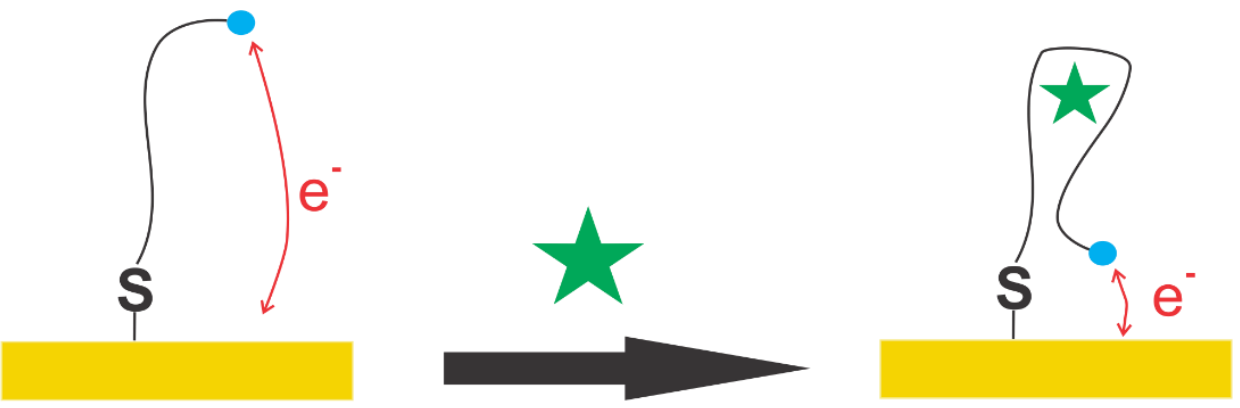

Signal on Redox label

(B)
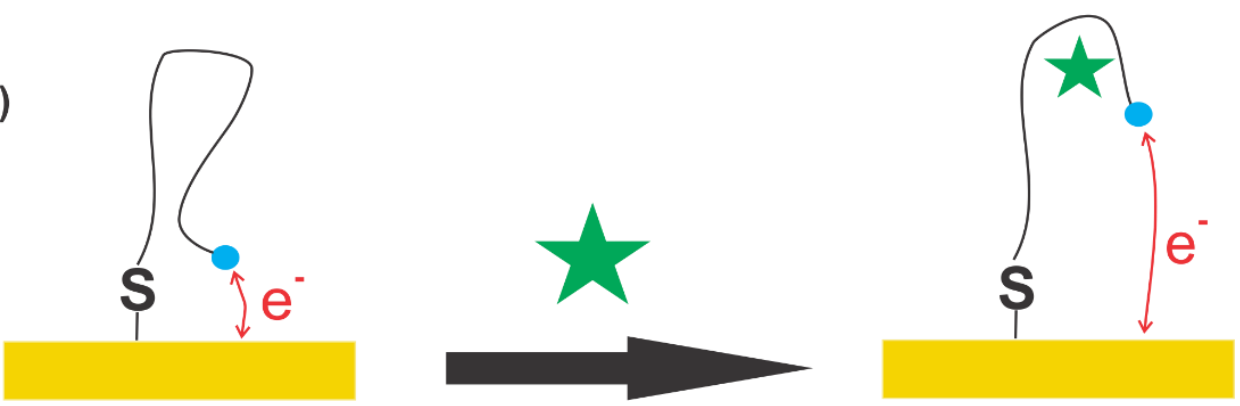

Signal off Redox label

Figure 1.11. Schematic representation of two voltammetry sensing strategies employing a methylene blue and thiolated modified aptamer. (A) Target induces a structural change of the ssDNA. The MB 
probe is getting closer to the electrode, and hence the current measured is increasing. (B) Target induces a structural change of the ssDNA. The MB probe is getting distant from the gold electrode, and thus the current measured is decreasing.

The main advantages of this class of transducer is the type of instrument used for these measurements, which is very easy to obtain and can be inexpensive and compact. However, the limitations for this signal transduction mechanism are the potential interferences to the response if the electroactive compounds generate false current values and, additionally, that the assay could be expensive due to the requirement of a redox label to generate the amperometric signal. Another limitation to this system is the potential disruption of the SAM formed due to the large potential range applied during the measurement.

\section{b. Potentiometry}

This technique measures the accumulation of a charge potential at the working electrode compared to the reference electrode in an electrochemical cell when no significant current flows between them. A potentiometric aptasensor based on chemically modified graphene (transducer layer) and aptamers (sensing layer) has been developed. ${ }^{100}$ The biosensor can detect the bacteria, Staphylococcus aureus, in a few minutes with high selectivity and simplicity. Zelada-Guillen et al. ${ }^{101}$ demonstrated a hybrid functional material carbon nanotubes/aptamer using the potentiometric transduction capabilities of single-walled carbon nanotubes in combination with the recognition capabilities of a protein-specific RNA aptamer.

\section{c. Field-effect transistor (FET)}

FET is a type of transduction that uses an electric field to control the conductivity of a channel between two electrodes in a semiconducting material. ${ }^{102}$ Zheng et al. ${ }^{73}$ demonstrated a $17 \beta$ Estradiol (E2) sensor based on an aptamer functionalized carbon nanotube network film fieldeffect transistors. The carbon nanotube is the conductive material where the aptamers are adsorbed on it. The device is enabling real-time detection of E2 in different liquids. Nakatsuka et al. ${ }^{56}$ developed a highly sensitive detection technique by using printed ultrathin metal-oxide FET modified with aptamers specific to serotonin, dopamine, or glucose. The main advantage 
of this measurement technique is its sensitivity; however, it is still a laboratory restrained technique with the need for complex materials and equipment.

\section{d. Electrochemical impedance spectroscopy (EIS)}

Electrochemical impedance spectroscopy (EIS) is now well-established as a powerful tool for investigating the mechanisms of electrochemical reactions, for measuring the dielectric and transport properties of materials, for exploring the properties of porous electrodes, and for investigating passive surfaces. ${ }^{71,72,103,104}$ In the field of biosensors, it is particularly well-suited to the detection of binding events on the transducer surface. Besides the detection of biorecognition processes, it is a valuable tool for characterising surface modifications, such as those that occur during the immobilisation of biomolecules on the transducer. The impedimetric detection for a biosensor is based on the determination of the charge transfer resistance Rct between the electrolyte containing a redox couple and the working electrode which is modified with the biorecognition molecules. These faradaic impedance measurements are usually performed in the presence of a redox couple in solution and rely on changes in the barrier to redox conversion due to the formation of the recognition complex itself or a subsequent complex. The ferri/ferrocyanide system is often chosen for this purpose. ${ }^{105,106} \mathrm{With}$ the use of a potentiostat directly linked to a three-electrode system (counter, reference, and working electrode), a direct current (DC) is applied and a small perturbation around the redox potential is created via the use of an alternating current (AC). The monitoring of the AC current as a function of frequency is providing information about the electron transfer between the solution and the electrode. The capability to explore the electrochemical system at relatively low frequencies using minimal perturbation serves to keep the kinetic information of the system under investigation at near-zero conditions (steady-state). Therefore, EIS is said to be a steadystate and non-destructive technique, on the contrary to most electrochemical techniques that use a large potential perturbation.

The acetamiprid has been detected via a highly selective EIS-based aptasensor. ${ }^{77}$ The EIS system was enhanced by electrodeposition of gold nanoparticles on the gold surface resulting in a higher active surface area which may improve the sensitivity of the detection. The electron 
transfer resistance increases upon target addition (signal-off represented in Figure 1.12B). A limit of detection of $1 \mathrm{nM}$ was reached.

The electron transfer resistance can decrease after target addition. Rodriguez et al ${ }^{107}$ showed a system to detect the lysozyme protein, where the combination of the protein with the aptamer produces a greater electron transfer between the solution and the working electrode resulting in a smaller Rct (Figure 1.12A).

\section{$\mathrm{Fe}(\mathrm{CN})_{6}^{3-14-}$}

(A)
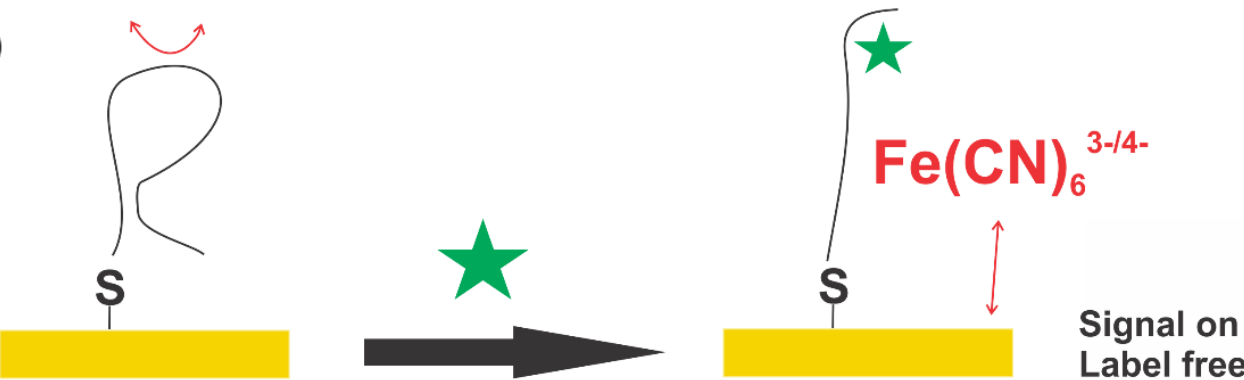

(B)
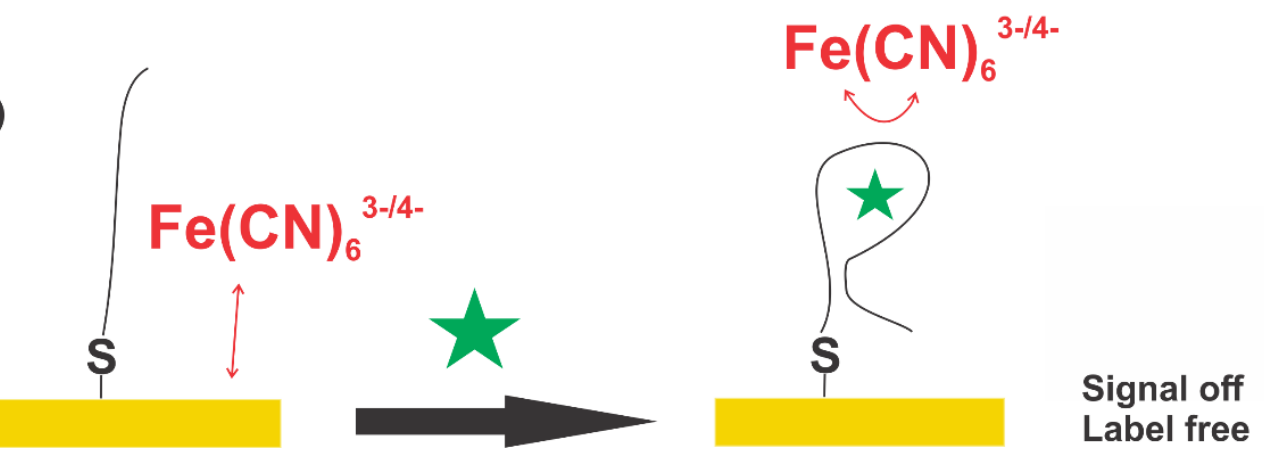

Figure 1.12. Schematic representation of two electrochemical label-free aptamer-based biosensor sensing strategies. (A) The target is inducing a structural change of the aptamer. The electron transfer resistance $R c t$, in this case, is decreasing upon target addition. (B) On the contrary to the previous case (A), the target addition is inducing an increase of the Rct.

The advantage of EIS is its sensitivity and its steady-state measurement. However, due to its sensitivity at the electrode surface, numerous spurious electrochemical signals can be monitored and consequently be interpreted as a specific signal. In the next section, a summary about the challenges regarding the aptamer characterization and aptasensors will be explored. 


\subsubsection{Aptamer characterization and aptasensors: challenges and opportunities}

Four main areas have been identified as current challenges in the small molecule detection using aptasensors. They are explored below:

Thermodynamics and structural information about the aptamer-ligand complex formation is required.

Complete structural characterizations were achieved for several aptamers such as cocaine aptamer, ${ }^{108-111}$ adenosine aptamer, ${ }^{112-115}$ thrombin aptamer, ${ }^{20,} 116,117$, and neomycin B aptamer. ${ }^{18,119}$ The binding site and the important nucleotide regions are observed, as well as the structural change of the aptamer upon aptamer-target complex formation. These aptamers showed great efficiency on different transduction platforms. ${ }^{63,98,99}$ Besides these well-studied aptamer-ligand complexes, numerous new aptamers are selected and produced for a great variety of ligands.

The dissociation constant $K_{D}$ has been assumed as a standard parameter for screening the efficiency of binding of the new aptamers due to its facility of acquisition and its simple physical interpretation. However, as described in Wilson et al., ${ }^{42}$ this last decade a pursuit of the lower $K_{D}$ started, and, therefore, newly produced aptamers have been mostly classified in the function of their $K_{D}$. Aptamers classified towards their $K_{D}$ involve three drawbacks for the future sensing of small molecules:

(1) High-affinity $K_{D}$ (low nanomolar range and lower) related to sensing experiments are not the most suitable aptamers because most of the time the applied detection range in a real sensing scenario is much higher than the $K_{D}$ and, consequently, the resulting saturated signal will not change over the desired detection range. Additionally, it will implicate more nonspecific interactions between the ligand and other parts of the aptasensor platform and, thus create more background signals to suppress.

(2) Common techniques to determine the $K_{D}$ use different additional chemicals (fluorescence probes, enzymes), different aptamer fixation methods (covalently fixed to an electrode, free in solution), and have a different level of sensitivity. ${ }^{36}$ As no "gold" standard method for the dissociation constant determination exists and is used similarly for each new aptamer selected, 
it is not coherent and appropriated to compare the $K_{D}$ acquired from different analytical methods.

(3) For therapeutics aptamer use where no transduction of the biological binding signal is needed, the affinity between the aptamer and its target is the major point to focus on. By contrast, for sensing experiments especially for the small molecule detection, the $K_{D}$ parameter must not be the priority among the binding parameters in comparison with the significance of the aptamer structural change. It will be difficult to transduce the biorecognition event in a physical signal without an important structural change of the aptamer upon the ligand addition. For instance, an aptamer selected for a small molecule with a $K_{D}$ determined with ITC at $5 \mathrm{nM}$ and presenting no significant structural change (lock-and-key or conformational-selection binding model) will be less compelling for sensing experiments than the same aptamer with a $K_{D}$ determined with ITC at $50 \mu \mathrm{M}$ presenting an important structural change (induced-fit binding model). A strong need to have a better understanding of the aptamer-ligand structural interactions emerges to avoid the misconception of always linked aptamer and important structural change and, thus, getting a piece of better knowledge on the aptamer-small molecule binding model. Additionally, the understanding of the binding mechanism may guide the choice of the most suitable transduction platform to use for an aptasensor.

\section{SAM stability is critical for the selection of an e-chem aptasensor.}

The electrochemical transduction platform appears as the most appropriate platform for an applied project of developing a sensing chip for allowing the detection of a specific molecule directly on the field. Most of the published works about electrochemical aptasensor platforms use already well-characterized aptamer (thrombin, adenosine, cocaine). ${ }^{63}, 98,99$ All these systems show a great binding signal transduced in an electrochemical signal. However, in the case of a modest structural change aptamer for a small molecule detection, the choice of the right electrochemical platform will be decided differently. Indeed, it is crucial first to get a stable electrochemical signal in a buffer solution before doing any sensing experiments. It is also important to have the lowest signal background on the e-chem used. Additionally, an amplification of the binding signal will be expected, it is worthy to use the platform in adequation with this purpose. 


\section{Limited stability and aptamer density on gold electrode with current SAM protocols.}

Electrochemical impedance spectroscopy (EIS) is a promising electrochemical transducer system for aptasensor technology. While its high sensitivity, its large amount of data acquired on the system, and its steady-state measurement, a signal drift related to buffer incubation occur with a self-assembled monolayer (SAM) composed of thiolated aptamer and backfiller molecules such as $\mathrm{MCH} .{ }^{120}$ A lack of understanding of the signal drift source is observed. The mechanism of SAM creation is a complex mechanism that involves different steps. ${ }^{86}$ Currently, the SAM studies were done mainly on short alkyl thiol molecules fixed on a gold electrode surface. By adding a long DNA strand to the overall SAM formation mechanism, some obstacles to the formation of a highly ordered SAM are created. The DNA strands are nitrogenrich molecules that have a strong affinity with the gold surface and, thus, a significant "lying down" conformation will appear at the gold surface. ${ }^{121}$ The impact of the tail-tail inter-DNA strands interactions can prevent the sulfur-gold chemical bond formation and, thus, impacting the aptamer density and the homogeneity of the SAM. Consequently, to all these obstacles, a highly disordered SAM and no control over the aptamer density occur. Some experiments/protocols of aptamer fixation must be explored and developed to fill the gap about the stability and the understanding of SAM formation using thiolated DNA strands on a gold electrode.

\section{Numerous spurious electrochemical signals are generated during sensing experiments.}

As presented in Liu et al., ${ }^{59,62}$ the use of a gold surface as the principal material for aptasensing experiments must be controlled due to the high affinity of some molecules to adsorb on it. Most of the aptasensor systems published had control experiments that focus on the selectivity of the aptamer. However, before proving the selectivity of the aptamer, it is important to understand the real behavior of the signal monitored. Especially in the small molecule detection field where the molecule due to its size can interact with numerous parts of the aptasensor system, and, consequently, create nonspecific signals that could be interpreted as a positive signal. In the context of a modest structural change aptamer with a small molecule target, specific and nonspecific signals are on the same scale and, therefore, a critical understanding of each signal observed must be achieved. A different response could be seen at a different level of ligand concentrations and each of these signals must be understood to create a reliable and trustful aptasensor. 


\subsection{Research problems and objectives \\ 1.2.1 Methamphetamine problem and current method of detection}

Methamphetamine (Erreur! Source du renvoi introuvable.) is a powerful, highly addictive stimulant used illicitly around the world. The illicit manufacture and use of methamphetamine are having a major impact on communities and individuals throughout New Zealand. Indeed, the New Zealand Police have identified over 1700 clandestine methamphetamine laboratories between 1999-2011, and the New Zealand Drug Harm Index in 2016 estimated 25,700 methamphetamine users. ${ }^{122}$ The reinvestment in organised crime per methamphetamine user is high and the total social costs (personal harm, community harm, and intervention costs) were NZ\$ 364.2 million in 2014. ${ }^{122,123}$

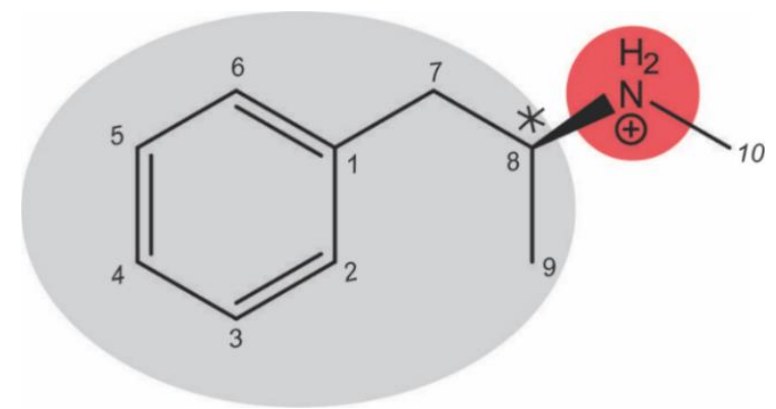

Figure 1.13. Skeletal representation of the methamphetamine molecule at $\mathrm{pH} 7.5$. The red shaded part is the cationic part of the methamphetamine due to the protonation of the imino group at $\mathrm{pH} 7.5$. The grey shaded part is an electron rich part which is mainly hydrophobic.

In addition to the known problems associated with illicit methamphetamine use, the clandestine synthesis of methamphetamine presents risks to the public from exposure to the sites where it has been manufactured. In New Zealand, these sites can be in residences, garages, or temporary accommodation such as hotels and motels. ${ }^{123}$ Because of factors such as odours or residues within the dwelling or property and the potential health problems of current occupants, the police and owners become suspicious of methamphetamine production at such sites.

Therefore, it is important to establish whether the manufacture of methamphetamine was likely to have occurred and the extent of any contamination due to this manufacture. The question is the following: what level of detection and methamphetamine residue on household surfaces 
pose a risk to human health. At this time, based on the findings in the CSA (The Prime Minister's Chief Science Advisor) report, ${ }^{123}$ if a housing NZ property test is between $1.5 \mu \mathrm{g}$ per $100 \mathrm{~cm}^{2}$ and $15 \mu \mathrm{g}$ per $100 \mathrm{~cm}^{2}$, decontamination is not triggered. When the level is above $15 \mu \mathrm{g}$ per $100 \mathrm{~cm}^{2}$, decontamination is needed to a level of $1.5 \mu \mathrm{g}$ per $100 \mathrm{~cm}^{2}$. The sampling is done with a wipe for a surface area of $100 \mathrm{~cm}^{2}$ then the wipe is placed in $3 \mathrm{~mL}$ of methanol. Related to this volume of collection, the methamphetamine concentration requirements are converted in $2.7 \mu \mathrm{mol} / \mathrm{L}\left(1.5 \mu \mathrm{g}\right.$ per $\left.100 \mathrm{~cm}^{2}\right)$ and $27 \mu \mathrm{mol} / \mathrm{L}\left(15 \mu \mathrm{g}\right.$ per $\left.100 \mathrm{~cm}^{2}\right) .{ }^{124}$

Because methamphetamine smoking usually results in much lower residue levels compared with methamphetamine manufacture, ${ }^{123}$ the distinction between manufacture and personal use needs to be established. Moreover, the manufacture may pose risks from several hazardous chemicals and by-products of the production of the drug. ${ }^{125}$ Based on the CSA report from the $29^{\text {th }}$ May 2018, New Zealand has spent a lot of money for decontamination even if the actual risk was not confirmed. The consequence of this action shows the high demand of new detection systems which can be used easily, quickly, and economically by the state.

The current method of testing and decontamination of a potentially contaminated dwelling is following the New Zealand Standards. ${ }^{126}$ After swiping a surface, the sample is taken to a specialized lab. Liquid chromatography coupled to mass spectrometry is used following a "gold" standard method. ${ }^{126}$ The current method is accurate and reliable, however, due to its large time-consumption, due to the need for highly trained personals and due to its cost, a new detection platform is required.

Methamphetamine aptamers have already been developed for aptamer-based biosensor systems. Ebrahimi et al. ${ }^{127}$ selected and used a methamphetamine aptamer with an electrochemical transduction platform. The aptamer strands are deposited on a gold electrode surface coated with AuNPs. The electron transfer resistance between the electrolyte solution and the gold surface is monitored upon methamphetamine addition.

On the other hand, Mao et al. ${ }^{128}$ developed a colorimetric aptasensor for the detection of methamphetamine. Au@Ag nanoparticles and magnetic beads are used. Nanoparticles are functionalized with a DNA reporter probe and the magnetic beads are conjugated with a DNA capture probe. DNA aptamers specific to the methamphetamine are designed specifically to hybridize at each end with the reporter and the capture probe. In solution, a "sandwich" is produced between nanoparticles, aptamers, and magnetic beads The methamphetamine 
addition induces disassembly of the "sandwich" formed resulting in a color change of the solution. A limit of detection of $0.5 \mathrm{nM}$ for the methamphetamine is reached. 


\subsubsection{Research objectives and thesis overview}

The principle aim of this project is to use a family of methamphetamine aptamers selected by AuramerBio (Wellington, New Zealand) for designing and developing an electrochemical aptamer-based biosensor sensing platform for methamphetamine detection. The research objectives will be separated into four different chapters:

- As mentioned in previous sections, the dissociation constant is valuable, however, for the sensing purpose of this thesis the physical interactions occurring between the aptamer and its target as well as the structural change of the aptamer upon target addition would be more valuable to comprehend. The objective of the Chapter $\mathbf{3}$ would be to unravel the binding model and the binding interactions between the methamphetamine and the aptamer through spectroscopic and calorimetric analytical techniques.

- With the aptamer chosen in Chapter 3, the most suitable e-chem transduction platform to the specific requirements of the level of detection $(2.7 \mu \mathrm{mol} / \mathrm{L})$, cost-effectiveness (lower cost than the current liquid chromatography analysis method), and simplicity of preparation and use (no need of additional chemicals for the preparation, lowest background signal of the electrochemical instrument and a non-expert person can do the analysis by itself), will be selected in Chapter 4 .

- The SAM formation is a complex mechanism especially when the use of thiolated single-stranded DNA is involved. A lack of knowledge concerning the SAM stability and the conformation of the DNA strand fixed occur. The first objective of the Chapter 5 objective would be to get a piece of better knowledge about the SAM stability by modifying the protocol of fixation, and thus creating a new SAM interface that will show different behavior. In the second part of the chapter, a protocol that would allow the enhancement of the detection signal for small molecules would be discovered and developed. The objective would be to combine stability and high aptamer density on the gold surface.

- Electrochemical aptamer-based biosensors for small molecule detection are suffering from a significant number of nonspecific interactions that create false positive signals. 
The complete investigation through a protocol that uses electrochemical impedance spectroscopy with different interferents molecules as well as negative aptamer would be used. The objective of the Chapter 6 would be to get sufficient knowledge on the different interactions occurring in the aptasensors system, and finally to assign the different electrochemical transduced signals observed into physical meanings. 


\section{CHAPTER 2}

\section{General methodology}

\section{Chapter overview}

In this chapter, the major theory of the different characterization techniques and electrochemical methods used in the thesis will be presented. For each section, the theory will be explored first, then some practical examples of each technique will be described.

\subsection{Characterization methods and instruments}

This section reviews the different characterization methods and instruments used for this thesis. Each will be described in the context of DNA analysis and DNA-ligand interaction analysis. A spectroscopic technique, called the circular dichroism, used to monitor the secondary structure of macromolecules such as proteins, RNA or DNA will be first presented. Secondly, a calorimetric instrument, called the isothermal titration calorimetry, affording the determination of the thermodynamic parameters of a receptor-ligand binding interaction will be described. Finally, nuclear magnetic resonance spectroscopy, which is a powerful technique to investigate with accuracy the protons in a molecule to get structural information, will be introduced. 


\subsubsection{Circular Dichroism (CD)}

The DNA structure is chiral and exists as a single enantiomer. This chirality is due to the building blocks that compose a DNA strand. Each nucleoside (nucleotide without a phosphate group) contains three centres of chirality. These carbons are part of the five-carbon sugar of each nucleoside. Moreover, the coupling of the stacked planar bases in DNA creates an asymmetry that makes the whole structure of DNA chiral (helical arrangement in space). ${ }^{41,129}$, 130

Chiral molecules are distinguishable by their interactions with polarized light at wavelengths near and distant from absorption energies, therefore, it is possible to analyse DNA molecules by using polarized light. ${ }^{131}$ The circular dichroism (CD) instrument measures the difference in absorbance by a chiral substance of right- and left-handed circularly polarized light (called ellipticity $\theta$ and expressed in degrees). ${ }^{41,129,132}$

The difference in absorbance is calculated as follows:

$$
\Delta A=A_{l}-A_{r}
$$

where $\Delta A$ is the difference in absorbance; and $A_{l}$ and $A_{r}$ represent the left absorption and the right absorption of the circularly polarized light.

The Beer-Lambert Law is rewritten as below:

$$
A=\left(\varepsilon_{l}-\varepsilon_{r}\right) c l
$$

where $\varepsilon_{l}$ and $\varepsilon_{r}$ represent the molar absorptivity of the left and the right circularly polarized light; $c$ is the molar concentration of the sample, and $l$ is the cuvette path length.

The difference in molar absorptivity is expressed as:

$$
\Delta \varepsilon=\varepsilon_{l}-\varepsilon_{r}
$$

where the elliptically polarized light is the superposition of the left circularly polarized light and the right circularly polarized light; and $\theta$ is the angle between the magnitude of the electric field vector at its maximum and its minimum. It is reported as follows: 


$$
\begin{gathered}
\theta=32.98 \Delta A \\
{[\theta]=3298 \Delta \varepsilon}
\end{gathered}
$$

where $\theta$ is the ellipticity in mdeg, and $[\theta]$ is the molar ellipticity in deg. $\mathrm{cm}^{2} \cdot \mathrm{dmol}^{-1}$. The molar ellipticity is circular dichroism corrected for concentration. ${ }^{133}$

Nucleoside chiral carbons only absorb below $190 \mathrm{~nm} .{ }^{134}$ With a CD instrument, the signal is measured between 190 and $300 \mathrm{~nm}$ and, thus, is due mainly to the structure chirality of the DNA strand. ${ }^{41,129,130}$ Therefore, the intensities and positions of the peaks for oligonucleotides in sigmoidal CD spectra are sensitive to the extent of base stacking and the orientations of dipole moments. ${ }^{56,130}$

In its most natural form, the DNA strand is structured as a right-handed helix. The term righthanded is related to the clockwise screw structure of the DNA strand. This structure is called the B-form structure. ${ }^{23,41,130}$ A typical spectrum of a DNA B-form structure is represented in Figure 2.1. A positive ellipticity is monitored at $280 \mathrm{~nm}$ and a negative at $245 \mathrm{~nm} .{ }^{41}$ The RNA natural form is structured as an A-form. ${ }^{41,130}$ The spectrum is like a B-form spectrum; however, the positive peak is shifted to $260 \mathrm{~nm}$ and the negative to $210 \mathrm{~nm}$.

DNA can also exist in a left-handed helix form and is called the Z-form structure. ${ }^{41,130}$ Other forms exist, and one that is especially compelling for sensing application, it is called the Gquadruplex structure. This structure can be formed only with a guanine rich DNA sequence. ${ }^{41}$, $129,130,135$
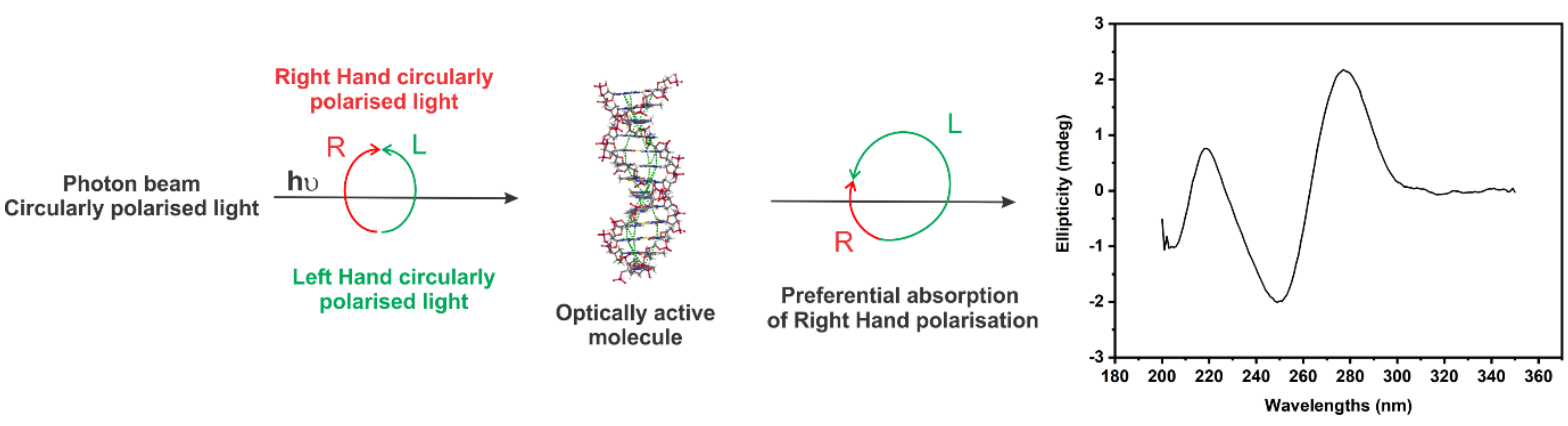

Figure 2.1. Schematic illustration of the analysis of an optically active molecule by circular dichroism. The data obtained are presented with a spectrum ellipticity $=f$ (wavelengths). 
As shown in Nakatsuka et al. ${ }^{56}$ the dopamine aptamer has an initial B-form structure. When dopamine is added to the solution, large changes in CD peak positions and relative intensities are monitored. The aptamer shifts away from predominant duplex signals (maxima at $280 \mathrm{~nm}$ ) and forms a new target-induced structural motif. A parallel G-quadruplex was suggested for the aptamer-dopamine complex. Similar findings are observed for the serotonin aptamer, where a shift and a change of intensity of the positive and negative peak of the initial B-form structure are induced by the addition of serotonin. ${ }^{56}$

Furthermore, the secondary structure of DNA is influenced greatly by temperature. By increasing the temperature of a triple helix DNA motif, the intensity of the two distinct peaks of the initial triplex structure decreases drastically. ${ }^{136}$ The DNA secondary structure is also sensitive to the divalent cation $\mathrm{MgCl}_{2}$ concentration. By increasing the concentration of $\mathrm{MgCl}_{2}$ in solution, Kypr et al. ${ }^{130}$ observed that the structure of a polynucleotide switch from a B-form to a Z-form structure.

\subsubsection{Isothermal Titration Calorimetry (ITC)}

Isothermal titration calorimetry (ITC) is an analytical calorimetric technique used for the study of intermolecular interactions. The ITC instrument measures the heat produced or consumed by the interaction between the receptor and its ligand during the titration. As the ITC experiment is performed at constant temperature and pressure, the information collected are related to the equilibrium association constant. In the normal ITC setup, the reference cell is filled with ultrapure water and the sample cell is filled with the receptor (protein, antibody, or aptamer). A syringe, filled with the ligand, injects at a constant time interval a specific volume of the ligand solution. A constant stirring during the titration is used in the sample cell to provide a good homogeneity of the reaction solution. Because the system is adiabatic, the difference in temperature between the reference cell and the sample cell is only related to the sample cell reaction. The power compensation needed to keep the sample cell at a similar temperature to the reference cell is converted into heat and a thermogram is collected (Figure 2.2). From the first law of thermodynamics, the change in the internal energy for a closed system is given by:

$$
\delta U=q-P \delta V
$$


where $\delta U$ is the change in the internal energy of a closed system; $q$ is the quantity of energy supplied to the system as heat, and $\mathrm{P} \delta \mathrm{V}$ is the amount of thermodynamic work done by the system on its surroundings.

The definition of the enthalpy is given by:

$$
H=U+P V
$$

The equation of a change in enthalpy is written as:

$$
\delta H=\delta U+P \delta V+V \delta P
$$

By combining Equation (2.6) and Equation (2.8) at constant pressure, we have the following:

$$
\delta H=q
$$

ITC measures the heat $q$ which is equivalent to the state function enthalpy, Equation (2.9) is the fundamental of ITC.

As observed in Figure 2.2, the binding affinity $K_{a}$, the enthalpy change $(\Delta H)$, and the stoichiometry of the reaction $n$ are deduced from the experimental part. The enthalpy change $(\Delta H)$ is directly read on the y-axis of the final graph as shown in Figure 2.2, the binding affinity is deduced from the slope of the tangency at the midpoint of the sigmoidal curve and the stoichiometry is deduced on the $\mathrm{x}$-axis at the midpoint of the sigmoidal curve. Then, the Gibbs free energy change $(\Delta G)$ and the entropy change $(\Delta S)$ are obtained from the Gibbs Equation (2.10) and the Lewis Equation (2.11):

$$
\begin{gathered}
\Delta G=-R T \ln \left(K_{a}\right) \\
\Delta G=\Delta H-T \Delta S
\end{gathered}
$$

where $R$ is the ideal gas law constant, and $T$ is the absolute temperature.

In the following discussion, a 1:1 binding reaction model between the receptor $\mathrm{R}$ and the ligand $\mathrm{L}$ to obtain the receptor-ligand complex RL is used and the reaction equation is given by: 


$$
R+L \leftrightarrow R L, \text { where } K a=\frac{[R L]}{[R] \times[L]}
$$

A relationship is observed in the Gibbs Equation (2.10), where the Gibbs free energy change and the binding affinity are linked. From Equation (2.12), a very large $K_{a}$ is related to a strong binding reaction between a receptor and a ligand. Therefore, a large negative $\Delta G$ is related to a strong binding reaction. All associations between a receptor and a ligand are driven towards a minimum of Gibbs free energy change. Thus, as observed from the Lewis Equation (2.11), a favourable enthalpy change is negative, and a favourable entropy change is positive.

The enthalpy change $(\Delta H)$ is related to the formation or disruption of energetically favourable interactions. A reaction is exothermic when $\Delta H<0$ (formation of interactions between atoms) and endothermic when $\Delta H>0$ (disruption of interactions between atoms). ${ }^{35,43,137}$ In a binding reaction, there are three different interactions to observe: receptor-ligand, receptor-solvent, and ligand-solvent. For instance, during a binding event, the formation of non-covalent interactions (H-bond or Van der Waals) between the receptor and the ligand $(\Delta H<0)$ can induce disruptions of non-covalent interactions between the receptor and the solvent and between the ligand and the solvent $(\Delta H>0)$. The overall enthalpy is then deduced.

The entropy change $(\Delta S)$ is a measure of how the heat energy is distributed over the overall thermodynamic system. It can also be viewed as a measure of the number of possible microscopic states of a system in thermodynamic equilibrium. The total entropy change of the system can be separated into three entropic terms:

$$
\Delta S=\Delta S_{\text {solv }}+\Delta S_{\text {conf }}+\Delta S_{r / t}
$$

where $\Delta S_{\text {solv }}$ is the solvent entropy change that results in solvent release from the surface of the receptor and/or the ligand upon binding. It often has a favourable contribution to the overall entropy change. ${ }^{45,52} \Delta S_{\text {conf }}$ is the conformational entropy change, which can contribute favourably or unfavourably because the degree of freedom of the complex formed may increase or decrease in comparison to the unbound receptor and ligand. ${ }^{35} \Delta S_{r / t}$ is the translational entropy change, which has mostly an unfavourable contribution to the overall entropy change due to the decrease of molecules number upon complex formation in the solution. ${ }^{138}$ 


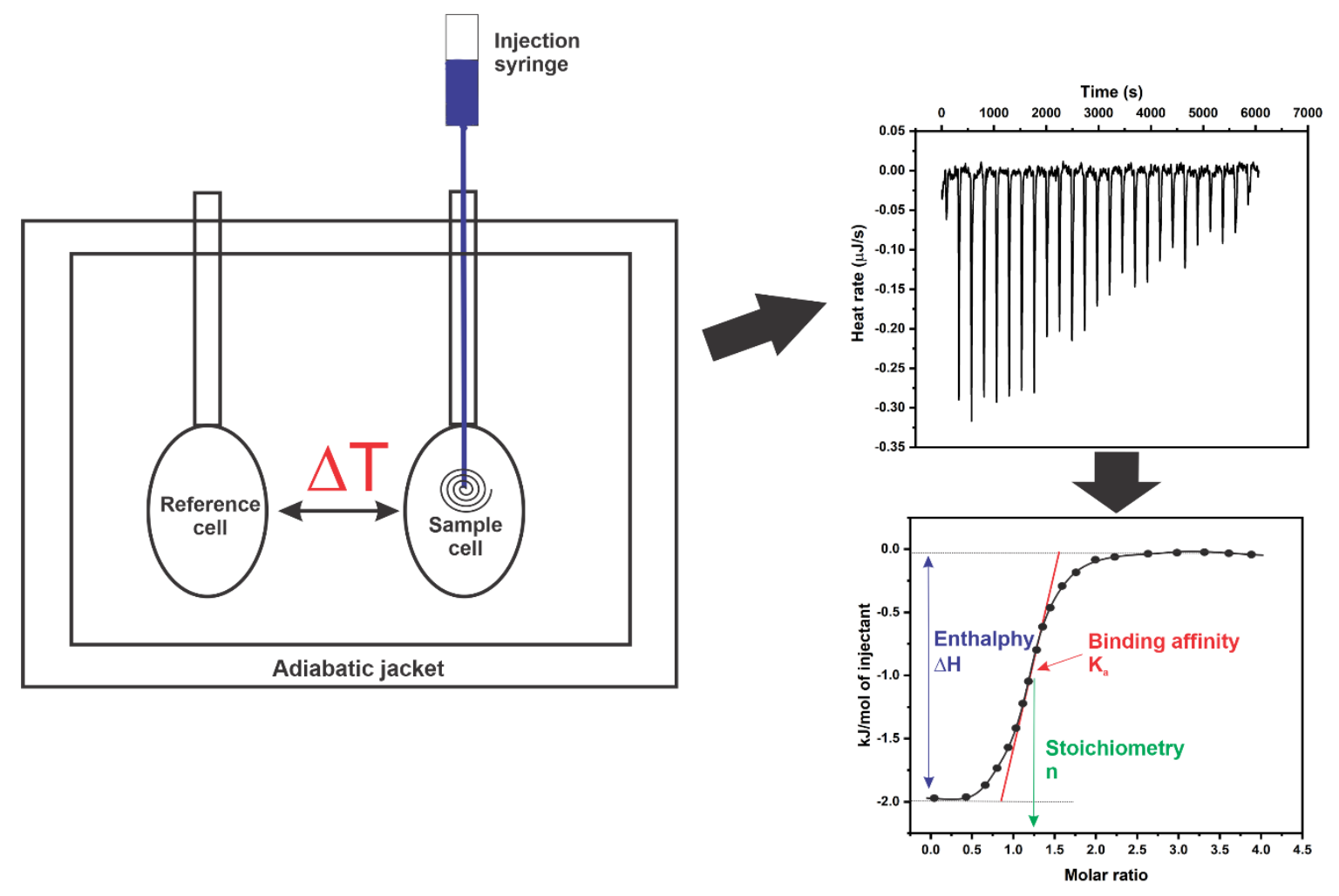

Figure 2.2. Schematic illustration of the isothermal titration calorimetry instrument. On the right, the raw data are obtained via a thermogram (Heat rate $\mu \mathrm{J} / \mathrm{s}=\mathrm{f}($ time $\mathrm{s})$ ). Finally, data are presented in a thermogram $(\mathrm{kJ} / \mathrm{mol}$ of injectant $=\mathrm{f}($ molar ratio $))$. From the final thermogram, the stoichiometry $n$, the enthalpy change $\Delta H$ and the binding affinity $K_{a}$ are deduced.

The cocaine aptamer has been investigated via ITC experiments. Neves et al. ${ }^{111}$ found a twosite ligand binding model at low salt concentration with the ITC instrument. ${ }^{111}$ The stoichiometry parameter of the aptamer-cocaine complex formation change upon salt concentration increases. Furthermore, mutation and truncation experiments can be carried out to monitor the change of the dissociation constant and, therefore, to observe the region of the important nucleotides. ${ }^{109}$ Zhang et al. ${ }^{115}$ did some experiments of designing new binding sites for the adenosine monophosphate (AMP) aptamer. The AMP aptamer is known to have two binding sites for the AMP molecule. By using the same binding site that they replicated into the sequence, they have been able to create an AMP aptamer with three and even four binding sites. The stoichiometry of the binding reaction is monitored with the ITC instrument. ${ }^{115}$ ITC experiments can also be used to determine a binding mechanism for the aptamer-ligand complex formation. ${ }^{35,39,108}$ The binding event is decomplexified to produce a quantitative 
evaluation of hydrogen bonding and electrostatic interactions with ITC experiments for the neomycin B aptamer. ${ }^{119}$

\subsubsection{Nuclear Magnetic Resonance (NMR)}

NMR is an analytical spectroscopic technique to obtain an atomistic view of the analysed sample. Nuclei samples are excited with radio waves in NMR and the magnetic nuclear spins are aligned along with an applied constant magnetic field $B_{0}$. The relaxation of the nuclear spins (from its excited state after $B_{0}$ to its original state) is called the resonance and differs for each magnetic nuclear spin depending on its original intramolecular magnetic field and its chemical environment. The resonance is expressed in part per millions (ppm) and is called the chemical shift $(\delta) .{ }^{139,140}$ The more the atom has a complex chemical environment the more it is shielded from the $B_{0}$ applied and the more it resonates at low frequency (upfield). On the other hand, the more the chemical environment of the atom is diminished the more it resonates at high frequency (downfield) because it is not protected from the $B_{0}$ applied. The most common atomic nuclei analyzed with NMR instrument are proton $\left({ }^{1} \mathrm{H}\right)$, carbon $\left({ }^{13} \mathrm{C}\right)$, nitrogen $\left({ }^{15} \mathrm{~N}\right)$, and phosphorus $\left({ }^{31} \mathrm{P}\right) .{ }^{141}$

NMR spectroscopy is a powerful tool to study nucleic acids in solution, where information about the structure, dynamics, and interactions with other molecules can be collected. For example:

(1) The base-pairing pattern, including Watson-Crick and non-Watson-Crick base pairs, can be predicted and verified. ${ }^{141-143}$

(2) Nucleotides assignment is possible. ${ }^{141-143}$

(3) The binding site of a DNA strand with a small molecule can be observed. ${ }^{141-143}$

(4) Conformational equilibria can be deduced. ${ }^{141-143}$

In the next section, the discussion will focus on ${ }^{1} \mathrm{H}$ NMR as this is the only NMR technique used during this thesis. As shown in Figure 2.3A, a ssDNA has numerous protons in its structure. These protons can be separated into different groups: $\mathrm{H}$ belonging to the five-carbon sugar, $\mathrm{H}$ attached directly to the heterocycle of each nucleotide, $\mathrm{H}$ belonging to the amino group involved and non/involved in base pairing, and $\mathrm{H}$ belonging to the imino group involved 
or non/involved in base pairing. Wijmenga and van Buuren ${ }^{142}$ published a comprehensive work where among others, they collected every chemical shift of each specific proton of a DNA strand.

As shown in Figure 2.3A, the ${ }^{1} \mathrm{H}$ NMR of a 40mer ssDNA is a complex spectrum. Indeed, the chemical shift dispersion is small because there are only four nucleotides. Furthermore, the Bform helix is the dominating secondary structure element found in DNA. Therefore, many nucleotides experience a similar chemical environment which causes similar chemical shifts. ${ }^{141}$ Big resonance clusters occur as observed around 2.5, 4, 6, and 7.5 ppm in Figure 2.3A. For example, the peak cluster around $4 \mathrm{ppm}$ represents the $\mathrm{H}$ belonging to the five-carbon sugar of the DNA strand. ${ }^{142}$

Among all these peak clusters, the region of resonance from 10 to $15 \mathrm{ppm}$ can be assimilated to the fingerprint region of the DNA strand, it is the region of the imino proton resonances of the guanine $(\mathrm{G})$ and the thymine $(\mathrm{T})$. These resonances are only observable when the imino protons are protected from the exchange with the bulk solvent water and are, therefore, involved in hydrogen bonding or structurally protected. Specifically, the imino protons in the resonance region of 12-15 ppm are involved in standard Watson-Crick base pairs (A-T or GC). Whereas, imino protons involved in non-Watson-Crick base pairs (or free in solution because protected from the solvent exchange effect) have upfield chemical shifts (10 -11.5 ppm). ${ }^{141,142}$

With the additional use of the Nuclear Overhauser Effect Spectroscopy (NOESY), which is a 2D NMR spectroscopic method, the base pair assignment is achievable. NOESY is a method based on the cross-relaxation of nuclear spins close to each other in space (cross-relaxation within $5 \AA$ ). Based on the observations of Nuclear Overhauser Effect (NOE) contacts, base pairs are identified. ${ }^{141,} 142$ In Figure 2.3B, a 2D NOESY spectrum of a DNA strand is represented. The correlation in space between different groups of protons is significant for the DNA strand. For example, as represented in Figure 2.3B the cluster at $\mathrm{x}=6 \mathrm{ppm}$ and $\mathrm{y}=4$ ppm shows that the sugar protons are close to the protons of the cytosine and thymine nucleotides in space. 

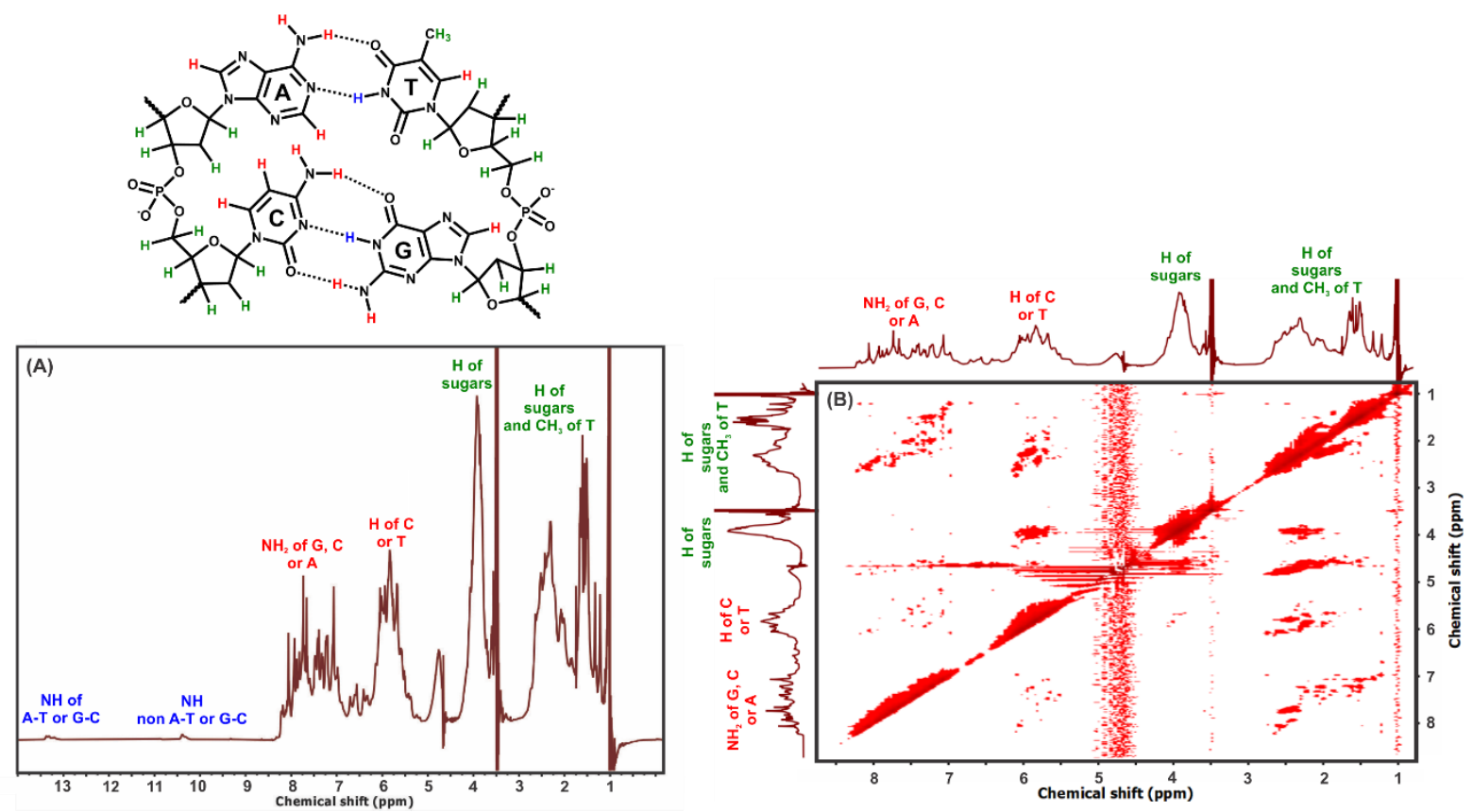

Figure 2.3. (A) Overall ${ }^{1} \mathrm{H}$ NMR spectrum and (B) Overall NOESY spectrum of a DNA strand. The protons belonging to the DNA strand can be separated in three groups related to the ${ }^{1} \mathrm{H}$ NMR resonance region.

The cocaine aptamer was studied thoroughly via the ${ }^{1} \mathrm{H}$ NMR technique. The secondary structure was confirmed with the assignment of base pairing in the 10-15 ppm pattern of the ${ }^{1} \mathrm{H}$ NMR spectrum. Furthermore, the cocaine-aptamer complex formation was monitored upon cocaine addition and a chemical shift mapping was done to observe the region of the important nucleotides for the binding event. The proton resonances that shift the more during the ligand addition are particularly compelling due to their proximity with the binding site. ${ }^{108-111,144}$

Complete characterizations with the acquisition of a Protein Data Bank (PDB) structure were done for several analyte-aptamer complexes such as thrombin, ${ }^{145,}{ }^{146}$ arginine, ${ }^{147}$ adenine monophosphate (AMP), ${ }^{148}$ neomycin $\mathrm{B},{ }^{118}$ theophylline, ${ }^{149}$ and tobramycin. ${ }^{150} .{ }^{1} \mathrm{H}$ NMR was used to monitor the enrichment of specific ssRNA during the SELEX process. The formation of new hydrogen-bonding through standard or non-standard Watson-Crick and new hydrogenbonding interactions between the aptamer and its analyte was monitored at each round of the SELEX in the resonance region of 10-15 ppm. With this coupling of ${ }^{1} \mathrm{H}$ NMR and SELEX, the choice of the ssRNA involved in a significant structural change is achievable. ${ }^{38}$ 


\subsection{Electrochemical methods}

This section reviews the different electrochemical methods used for this thesis. In the following section, all electrochemical techniques are based on a three-electrode system with a reference, a working, and a counter electrode all connected to a potentiostat (Figure 1.8). First, the electrochemical voltammetry measurement is introduced followed by the presentation of the main voltammetry experiments used for this thesis (cyclic voltammetry (CV), square wave voltammetry (SWV), and differential pulse voltammetry (DPV)). Then, the theory and practical aspects of the electrochemical impedance spectroscopy (EIS) method will be described. Finally, the chronocoulometry method will be presented.

\subsubsection{Voltammetry (CV, DPV, and SWV)}

In electrochemical voltammetry experiment, the potential of the system is controlled, and the current is measured in the function of the time. The potential of the WE is controlled with respect to the reference electrode and the current at the WE is measured. The applied potential results in the oxidation or reduction of solution phase species, which changes the local concentration of species in the solution near the electrode surface. The electroactive species can be found directly in solution (for instance the redox couple ferri/ferrocyanide dissolved in solution) or it can be found fixed at the end of a biorecognition element that is fixed on the working electrode (for instance the methylene blue molecule). Voltammetry measurement offers various advantages: the sensitivity and the linear concentration range is excellent $\left(10^{-12}\right.$ to $10^{-1} \mathrm{M}$ of electroactive species); the analysis time is rapid, and the method can be used with a wide range of solvents and electrolytes. In addition, valuable information about kinetics (electron transfer) reaction can be obtained..$^{71,72}$

- Faradaic vs non-faradaic current

As explained above, the desired current to monitor is the one that implies an ion or atom which is reduced or oxidized to another species, and in addition, the reactant must come from outside the electrode, and the product of the reaction must leave the electrode again, the resulting current is called faradaic. ${ }^{151,152}$ In parallel, all currents observed at the electrode surface that are not attributed to the redox process are called non-faradaic current. A major example of a 
non-faradaic current in electrochemical experiments is the charging process of the electrode surface resulting in an important capacitance parameter that can interfere with the electrochemical measurement.

\section{Cyclic Voltammetry (CV)}

Cyclic voltammetry consists of scanning linearly the potential of a working electrode (WE) using a triangular potential waveform. A first scanning potential is chosen for the first half of the cycle, usually starting from a potential value where no reaction occurs. As the applied potential approaches to the characteristic standard reduction potential $\left(E^{\circ}\right)$ for the redox process, an anodic current begins to increase until a peak is reached. After passing the potential region where the oxidation process takes place, the potential sweeping direction is reversed. During the reverse scan, the oxidized molecules (generated in the forward scan, and accumulated near the electrode surface) are reduced and a cathodic peak is observed. ${ }^{71,72,153}$

The CV method is used in the thesis for electrode cleaning and the control of the cleanliness of the electrode. ${ }^{154,155}$ These experiments are described in the following section to have a more practical understanding of the $\mathrm{CV}$ technique.

\section{a. Electrochemical cleaning of gold electrode via CV}

The fixation of thiol ssDNA on the gold surface is used for most of the sensing experiments. After each final step of the experiments, the gold electrode is still modified with numerous strong bonds Au-S. To have an efficient electrode cleaning, the first cleaning step will be to disrupt and break these strong bonds previously created. The use of cyclic voltammetry with a non-faradaic solution of sodium hydroxide $(0.1 \mathrm{M} \mathrm{NaOH})$ was chosen for the electrochemical desorption of the remaining self-assembled monolayer. ${ }^{82,86,156}$ As observed in Figure 2.4, the negative current peak at $-1.2 \mathrm{~V}$ is a typical desorption potential for n-alkanethiolates. ${ }^{82,156}$ The peak is not observed on the final CV scan in sodium hydroxide solution (Figure 2.4). Manual polishing is done after each step of sodium hydroxide cleaning to ensure the removal of any remaining covalent bonds. 


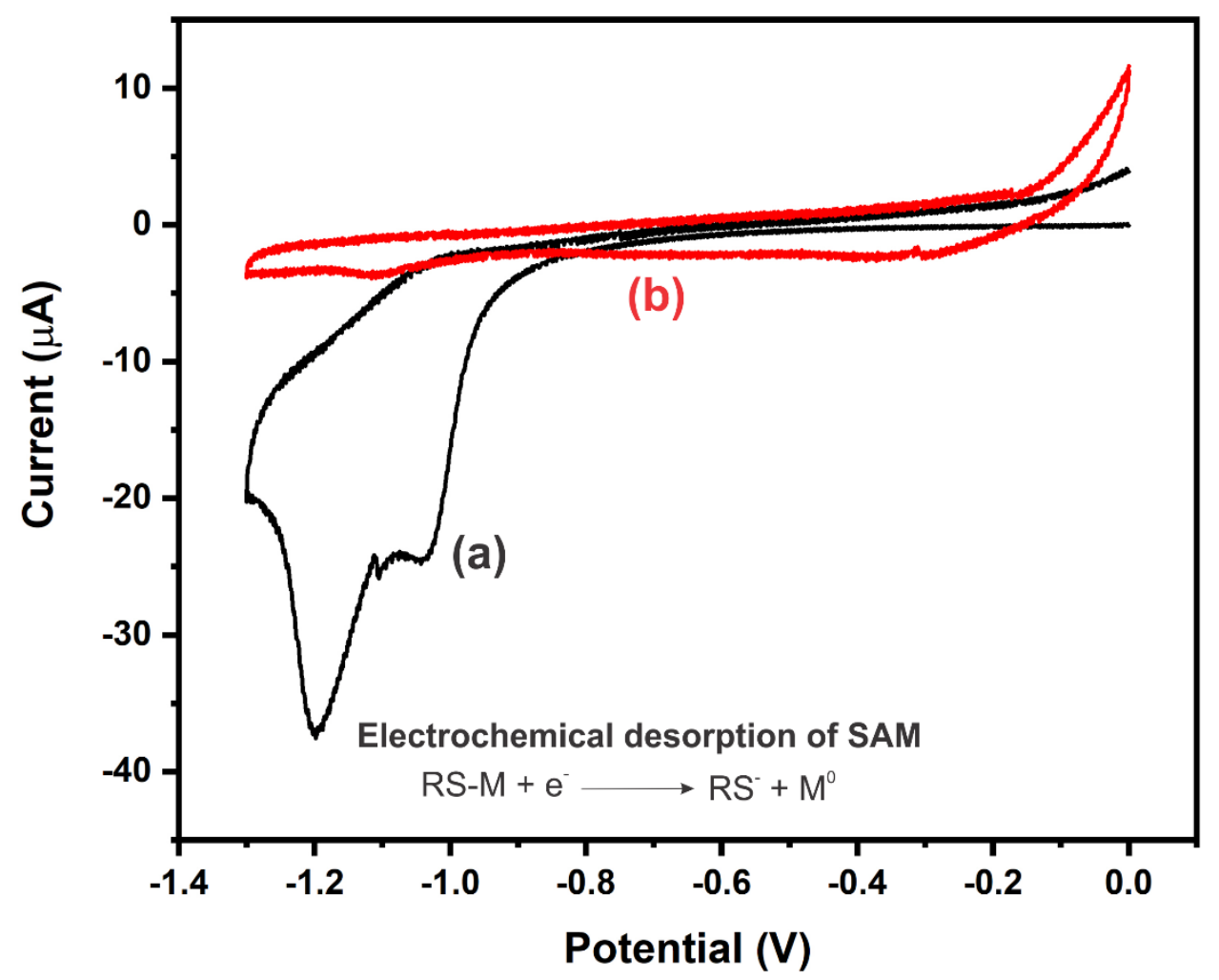

Figure 2.4. Cyclic voltammograms of bare gold electrode after electrochemically cleaning in $0.1 \mathrm{M}$ $\mathrm{NaOH}$ at $1 \mathrm{~V} / \mathrm{s}$ scan rate: (a) scan 1; (b) scan 200. The reference electrode used is a $\mathrm{Ag} / \mathrm{AgCl}$ (3 M $\mathrm{KCl})$.

After the manual polishing step, a third cleaning method is used to ensure the cleanliness of the electrode surface. The gold surface is electrochemically cleaned with an acid solution 0.5 M sulfuric acid $\left(\mathrm{H}_{2} \mathrm{SO}_{4}\right){ }^{155}$ Through the $\mathrm{CV}$ spectrum shape and the reproducibility of the $\mathrm{CV}$ scans, the cleanliness of the electrode will be partially confirmed. In addition, a roughness factor can be determined used for the real surface area calculation of the electrode.

The working electrode is a polycrystalline gold electrode, by placing it in a sulfuric acid solution and doing a cyclic voltammetry experiment, oxidation and reduction reactions of the gold layer are generated at the electrode surface. As observed in Figure 2.5, two peaks of oxidation and one peak of reduction appear during the $\mathrm{CV}$ measurement. The oxidation peaks are related to the oxide layer formation $\left(\mathrm{Au}_{2} \mathrm{O}_{3}\right)$ on the electrode surface. 
The reduction peak is related to the reduction of the oxide layer $\left(\mathrm{Au}_{2} \mathrm{O}_{3}\right)$ previously formed inAu. The reduction peak (Figure 2.5, black shaded area) obtained identifies an important piece of information related to the surface roughness of the electrode. ${ }^{154}$ In fact, the gold electrode external interface must be viewed as an important heterogenic surface containing defects, metal film impurities, occlusions, and other gross structural irregularities. ${ }^{82}$ This roughness factor must be used for the real surface area calculation for further aptamer density calculations. As the reduction peak represents the amount of surface oxide formed, the calculation of the roughness factor and the real surface area can be made with the integrated value of the reduction peak. Indeed, the calculation assumes that a monolayer of chemisorbed oxygen with a gold: oxygen ratio of 1:1 has been formed. Thus, the charge associated with the formation or reduction of the gold surface $\left(Q_{0}\right)$ is related by:

$$
\mathrm{Q}_{0}=2 \mathrm{eN}_{\mathrm{A}} \Gamma_{0} \mathrm{~A}
$$

where $N_{A}$ is the Avogadro constant $\left(6.022 \times 10^{23} \mathrm{~mol}^{-1}\right) ; e$ is the elementary electric charge $\left(1.602 \times 10-{ }^{19} \mathrm{C}\right) ; \Gamma_{0}$ is the surface concentration of atomic oxygen assumed to be equal to the surface density of gold atoms, and $A$ is the real surface area $\left(\mathrm{cm}^{2}\right)$. The equation (2.14) can be shortened by assuming that $2 e N_{A} \Gamma_{0}=Q^{*} \cdot{ }^{154,157}$ Therefore, equation (2.15) can be written as:

$$
A=\frac{Q_{0}}{Q^{*}}
$$

where $A$ represents the real surface area; $Q_{0}$ the charge associated with the reduction of the gold oxide layer, and $Q^{*}$ the reference value for the charge required for the reduction of an oxygen monolayer chemisorbed on polycrystalline gold. ${ }^{154,157}$

The reference value $Q^{*}$ for the polycrystalline Au layer found by Oesch and Janata is 482 $\mu \mathrm{C} / \mathrm{cm}^{2} .154,157$ 


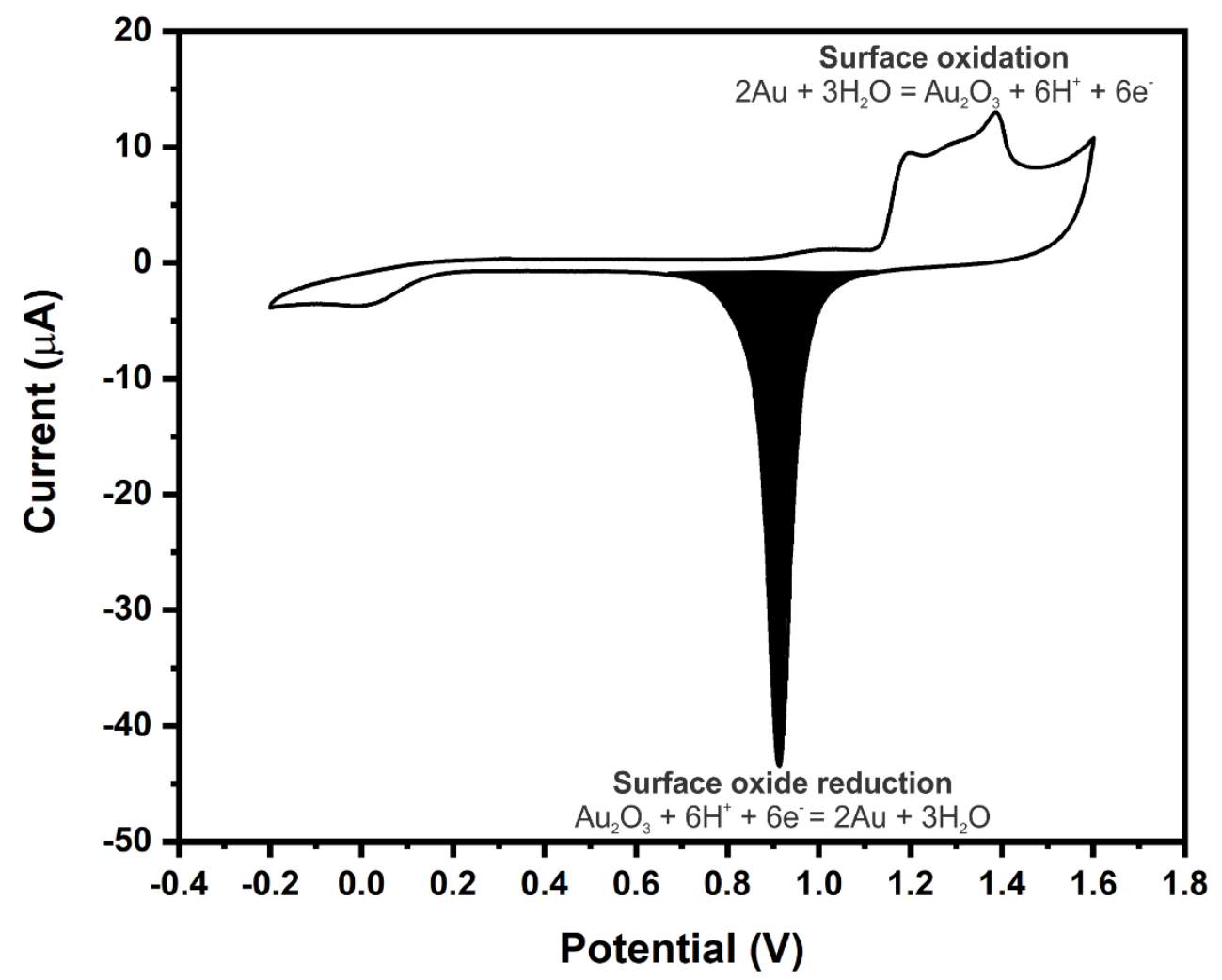

Figure 2.5. Typical cyclic voltammogram of bare gold electrode after electrochemically cleaning in 0.5 $\mathrm{M} \mathrm{H}_{2} \mathrm{SO}_{4}$ at $0.1 \mathrm{~V} / \mathrm{s}$ scan rate. The reduction peak area (black shaded part) represents the amount of gold oxide formed. The reference electrode used is a $\mathrm{Ag} / \mathrm{AgCl}(3 \mathrm{M} \mathrm{KCl})$.

\section{b. Control of the cleanliness of the gold electrode via CV}

A last use of $\mathrm{CV}$ measurement is the cleanliness checking of the electrode surface by using a redox couple (the ferri/ferrocyanide solution). The electron transfer involved in the redox reaction is an important parameter to monitor. Theoretically, for a single-electron transfer reaction such as in the ferri/ferrocyanide couple on a perfect gold surface, the potential difference between the reduction and the oxidation peak is $59 \mathrm{mV}$ at $25^{\circ} \mathrm{C} .{ }^{71}$ This value is used as a "gold standard" for cleaning monitoring. A standard CV of a clean gold electrode surface in ferri/ferro solution is shown in Figure 2.6. 


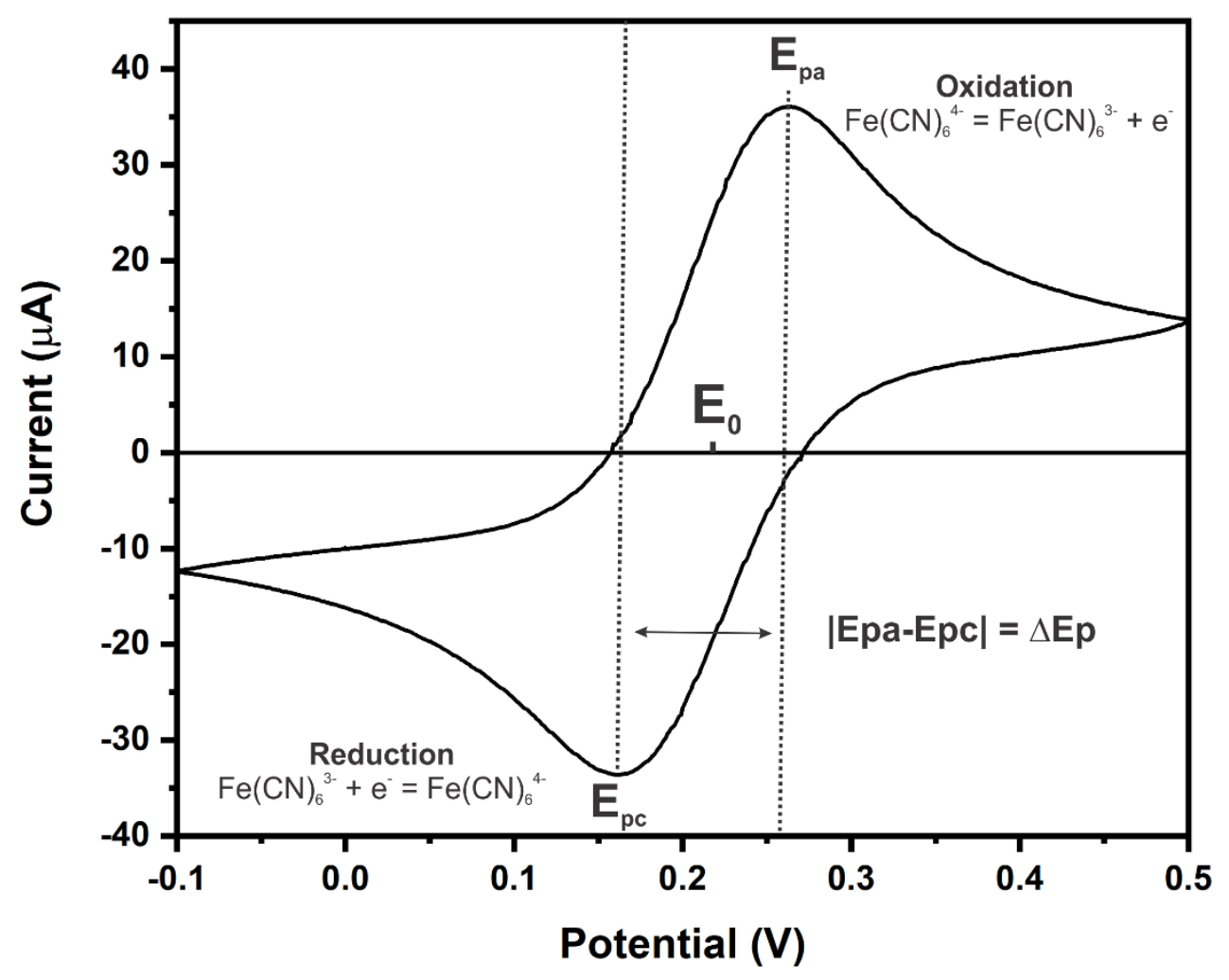

Figure 2.6. Cyclic voltammogram of bare gold electrode in $5 \mathrm{mM}$ ferri/ferrocyanide solution. $E_{0}$ is the specific redox potential of the ferri/ferro couple. $E_{p c}$ and $E_{p a}$ are representing the cathodic (reduction) potential and anodic (oxidation) potential. The reference electrode used is a $\mathrm{Ag} / \mathrm{AgCl}(3 \mathrm{M} \mathrm{KCl})$.

\section{Differential Pulse Voltammetry (DPV) and Square Wave Voltammetry (SWV)}

One of the major drawbacks related to $\mathrm{CV}$ measurement is the non-faradaic current monitored along with the faradaic current. No separation of both currents is possible, consequently, it could create some interferent electrochemical signals. In voltammetry, other techniques such as DPV and SWV have been developed to overcome this limitation and isolate only the faradaic current. DPV and SWV are part of the pulse voltammetry methods family. ${ }^{71,72}$ Unlike the CV measurement where the potential is varied linearly with time (as observed in Figure 2.7C, the applied signal is a voltage ramp), a regularly increased pulse height is applied at periodic intervals for DPV and SWV. However, the shape of the applied potential in the function of time is different for DPV and SWV (Figure 2.7). 
DPV is a technique where fixed-magnitude pulses $(\Delta E p)$ are superimposed on a linear potential ramp. As observed in Figure 2.7A, the response current is sampled twice, just before the pulse application $\tau^{\prime}$ and again late in the pulse life $\tau$. The current record is a plot of the current difference (at $\tau^{\prime}$ and at $\tau$ ) versus the base potential. ${ }^{71,153}$

SWV is a large-amplitude differential technique in which a wave form composed of a symmetrical square wave, superimposed on a base staircase potential, is applied to the working electrode. A pulse amplitude $\left(E_{s w}\right)$ is used. As in DPV, the sample is sampled twice during each-square wave cycle, once at the end of the forward pulse $\left(i_{\text {forward }}\right)$ and once at the end of the reverse pulse $\left(i_{\text {backward }}\right)$. The current is recorded as a net response of the difference between the two monitored currents (Figure 2.7B). In comparison to DPV, a better sensitivity occurs because the net current is larger than either the forward or the reverse components (since it is the difference between them); in DPV the reverse current is not used. ${ }^{71}{ }^{158}$ Similar to CV measurement in faradaic systems, a faradaic current is monitored via a redox couple or a redox molecule in solution. 

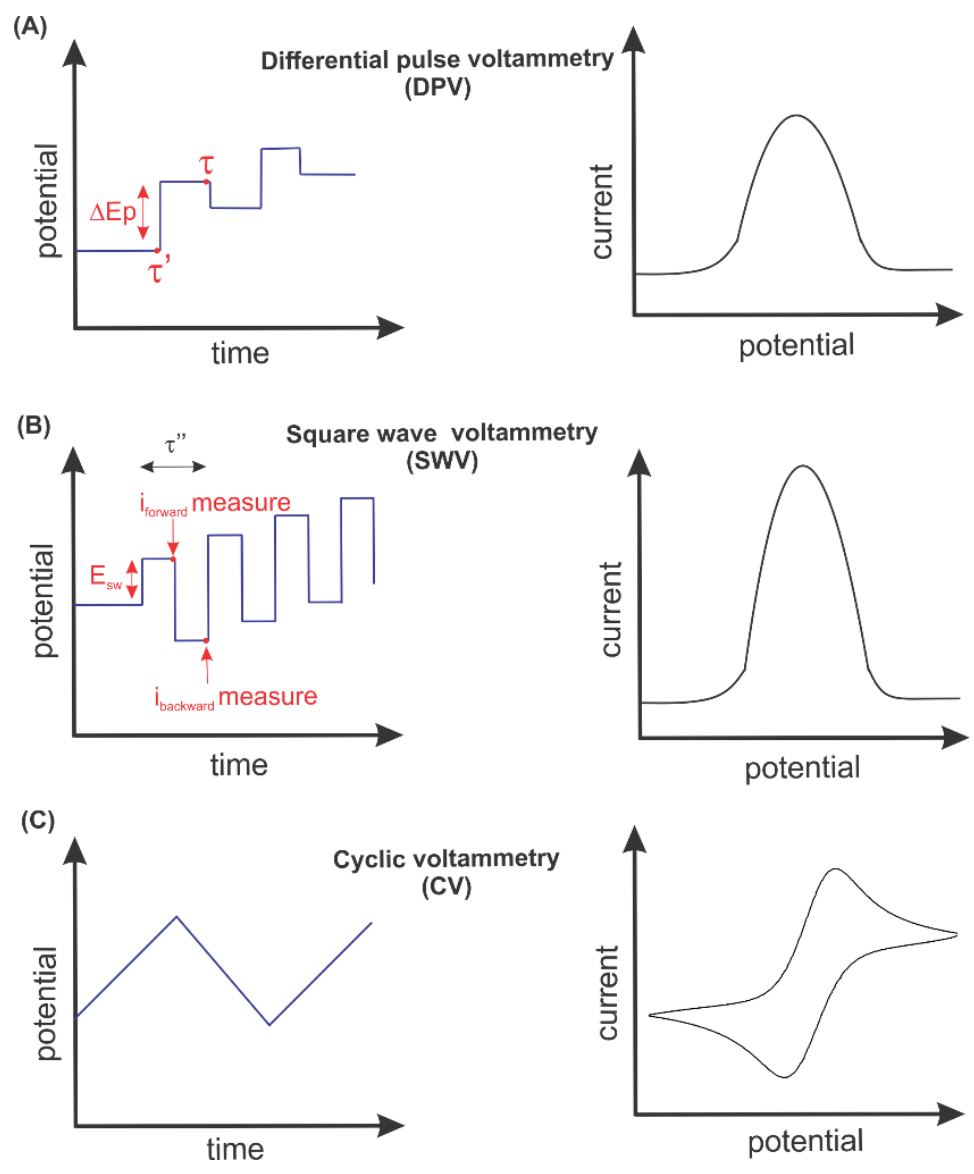

Figure 2.7. Schematic representations of potential vs time and current vs time for three voltammetry techniques. (A) Differential pulse voltammetry (B) Square wave voltammetry and (C) Cyclic voltammetry.

\subsubsection{Electrochemical Impedance Spectroscopy (EIS)}

Voltammetry techniques presented previously involve large perturbations on the system. By imposing potential steps or potential sweeps, the electrode is driven to a condition far from equilibrium. ${ }^{71,72}$ The basis of the electrochemical impedance spectroscopy (EIS) technique is to perturb the system with an alternating voltage $\left(E_{A C}\right)$ of small magnitude and to observe how the system follows the perturbation at steady state. Many advantages appear for this technique: (1) the possibility to make high-precision measurements because the response may be indefinitely steady and therefore be averaged over a long term, (2) Because the measurements are done over a wide frequency range, the information can be separated into kinetics and diffusion terms, and (3) it is a non-disruptive measurement method. ${ }^{71,72,103,104,159}$ 
For an ideal resistor, Ohm's law is given by

$$
R=\frac{E}{I}
$$

where $R$ is the resistance $(\Omega) ; E$ is the voltage $(V)$, and $I$ is the current $(A)$.

The EIS system is a much more complex system and the resistance must be replaced by the impedance. Like resistance, impedance is a measure of the ability of a circuit to resist the flow of electrical current. Electrochemical impedance is measured by applying an alternating current (AC) potential (sinusoidal potential excitation) centred around the direct current (DC) potential (which is the specific redox potential of the faradaic component used for the EIS experiment) and then measuring the current through the cell. As the excitation potential is sinusoidal, the current response is sinusoidal but shifted in phase $\varphi$ and magnitude $I_{0}$ (Figure 2.8A). To create a small sinusoidal perturbation, a frequency range is used.

The EIS experiment is a faradaic impedance measurement in which the cell contains a solution with both forms of a redox couple (here the ferri/ferro couple), so that the potential of the working electrode is fixed $\left(E_{D C}\right)$. The impedance measurement is related to the electron transfer between the redox species and the working electrode (faradaic current) and to the charge and discharge of the double layer (Figure 2.8B) at the working electrode/electrolyte interface (nonfaradaic current).

To simplify the data acquired (separation between faradaic and non-faradaic data), electrical equivalent circuits (EECs) are used to approximate the experimental impedance data (Figure 2.8C). The EEC model used for this investigation is the Randles circuit. ${ }^{71,72,159}$ Four components are included in this model: the solution resistance $R_{s}$, the charge transfer resistance $R c t$, the capacitance $C_{d l}$ or constant phase element CPE, and the Warburg impedance $W$.

The faradaic component arises from the electron transfer via a reaction across the interface by overcoming an appropriate activation barrier, namely the charge transfer resistance $R c t$, along with the uncompensated solution resistance $R_{S}$ (high-frequency component). The non-faradaic current results from charging the double-layer capacitor $C_{d l}$ and from the mass transport of the reactants, the Warburg diffusion element $W$ (low-frequency component represented by a straight line with a phase of $45^{\circ}$ in the Nyquist plot). 
(A)

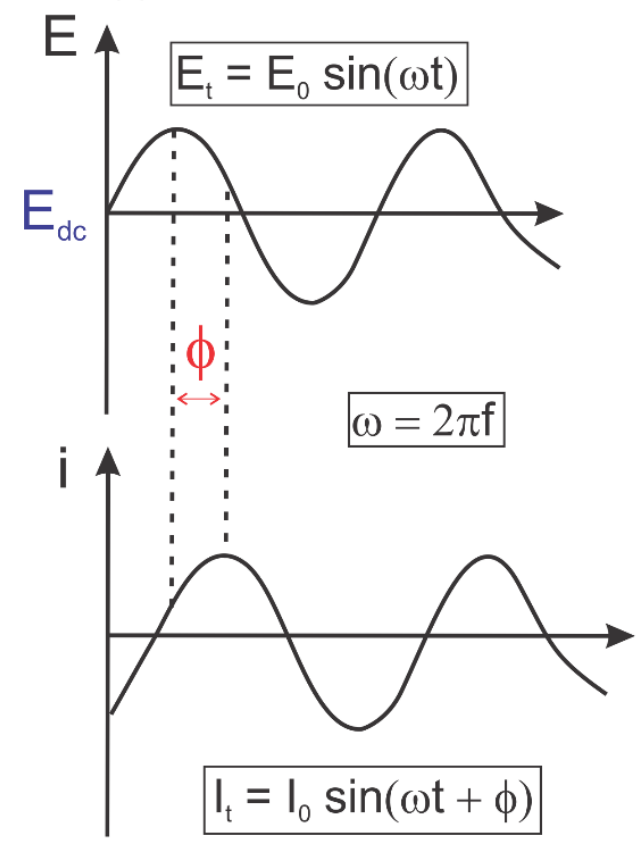

(B)

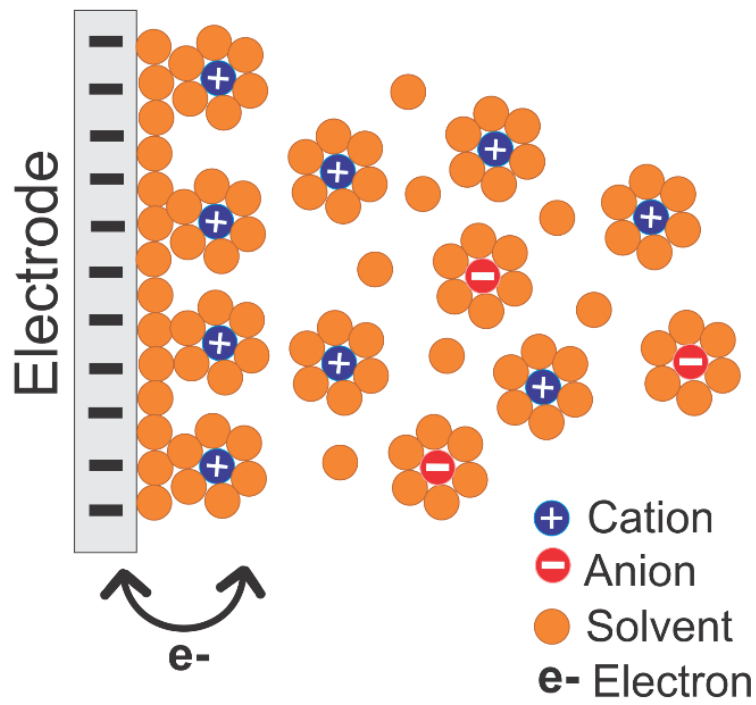

(C)

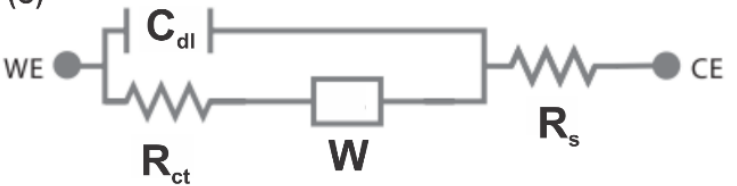

Figure 2.8. (A) Voltage vs time and current vs time represented for an electrochemical impedance spectroscopy measurement. (B) An electrified interface is represented in which the electrode is negatively charged; the countercations are aligned along the electrified surface. (C) The electrical circuit elements corresponding to each interface component is shown.

As shown in Figure 2.8A, the voltage and the current can be written in the function of the time. So, the impedance can be written as

$$
Z(t)=\frac{E(t)}{I(t)}=\frac{E_{0} \sin (2 \pi f t)}{I_{0} \sin (2 \pi f t+\Phi)}
$$

As observed in Equation 2.17, the impedance is a complex value because the current can differ in terms of the amplitude and additionally, shows a phase shift $\varphi$ in comparison to the $E(t)$ function. ${ }^{104}$ Thus, impedance can be written as an addition of a real impedance part $(\operatorname{Re} Z)$ and an imaginary impedance part (ImZ) as follows:

$$
Z(t)=R e Z+j \operatorname{Im} Z \text { where } \mathrm{j}=\sqrt{-1}
$$

The data is commonly presented by the Nyquist plot as shown in Figure 2.9. From the Nyquist plot, the values of $R s$ and $R c t$ can be easily determined. The double-layer capacitance can be 
calculated from the frequency at the maximum of the semicircle. The $45^{\circ}$ line indicating Warburg-limited behaviour at a frequency close to 0 .

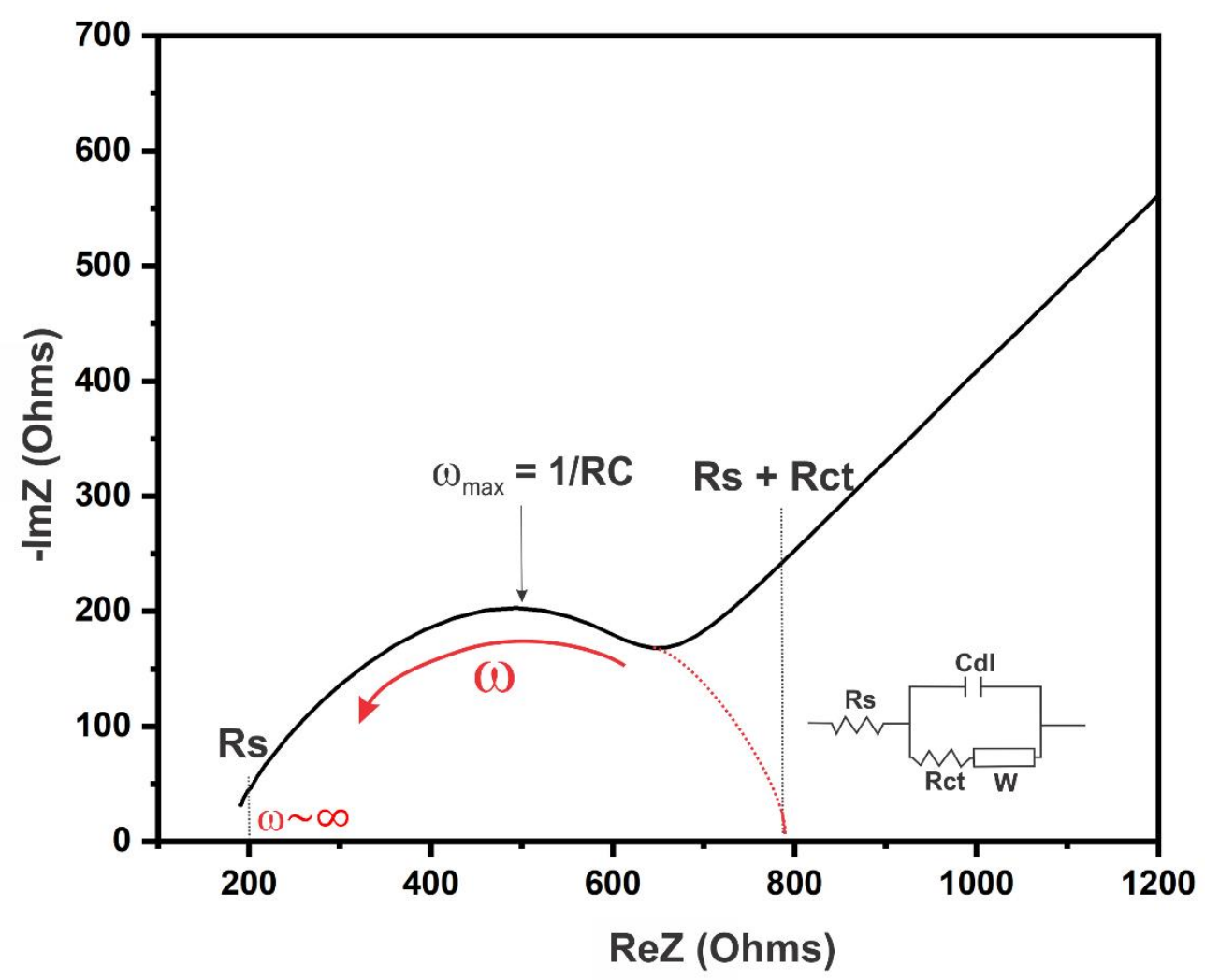

Figure 2.9. Nyquist plot representation. EIS data are obtained through this Nyquist plot. At frequency ( $\omega$ ) close to 0 , the mass transfer $\mathrm{W}$ component is predominant, and the phase shift is $45^{\circ}$. At high frequency, the solution resistance $\mathrm{R}_{\mathrm{s}}$ component is predominant.

\subsubsection{Chronocoulometry (CC)}

The calculation of the DNA surface density on an electrode surface is achieved via chronocoulometry measurement. Indeed, the CC experiment involves the measurement of the charge as the function of the time response to an applied potential step at the working electrode (Figure 2.10B). The potential stepping takes place from a value where no reaction occurs to a value that leads to electrolysis (oxidation or reduction) of the electroactive species. ${ }^{160}$ The 
Cottrell equation describes the relationship between the charge $(Q)$ of the surface-confined redox species and the time $(t)$ in an electrochemical system:

$$
Q(t)=\frac{2 n F A C_{0} \sqrt{ } D_{0}}{\sqrt{\pi}} \times \sqrt{t}+Q_{d l}+n F A \Gamma_{0}
$$

where $n$ is the number of electrons; $F$ the Faraday's constant; $A$ the electrode surface area; $D_{0}$ the diffusion coefficient; $C_{0}$ the bulk concentration; $Q_{d I}$ the capacitive charge; $n F A \Gamma_{0}$ is the charge from the reduction of redox marker on the electrode, and $\Gamma_{0}$ represents the amount of redox marker on the electrode surface. ${ }^{160}$

When $t=0$, the detected charge is the sum of $Q_{d l}$ and the surface excess terms, $n F A \Gamma_{0}$. Capacitive charge $\left(Q_{d l}\right)$ and $n F A \Gamma_{0}$ that quantifies the charge of the adsorbed electro-active species pass through very quickly in comparison to the slow diffusion component of the bulk and therefore can be separated in a plot of the total charge $Q$ vs. $t^{1 / 2}$ (Figure 2.10A). Hence, $Q_{d l}+n F A \Gamma_{0}$ values are obtained from the intercept. Surface excess $\left(\Gamma_{0}\right)$ of the adsorbed electroactive species is separated from $Q_{d l}$ by performing an additional CC experiment in the supporting electrolyte that lacks the electro-active species. The surface excess of redox marker is converted to DNA probe surface density with the relationship:

$$
\Gamma_{D N A}=\Gamma_{0}\left(\frac{\mathrm{z}}{m}\right) \mathrm{N}_{A}
$$

where $\Gamma_{D N A}$ is the surface density in molecules $/ \mathrm{cm}^{2} ; m$ is the number of bases of the DNA sequence; $z$ is the charge of the redox molecule, and $N_{A}$ is Avogadro's constant.

Here, the use of a cationic redox marker is necessary because DNA doesn't present quantifiable electro-activity. $\mathrm{Ru}\left(\mathrm{NH}_{3}\right)_{6}{ }^{3+}(\mathrm{RuHex})$ is used and it will exchange for native counterions associated with the DNA phosphate. The amount of redox marker electrostatically attached to the DNA is then determined using chronocoulometry as described at the beginning of this section. 
(A)

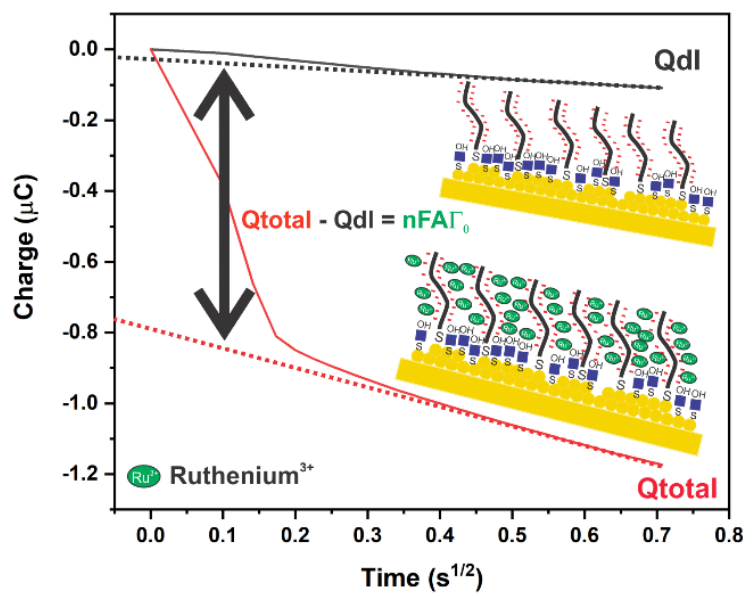

(B)
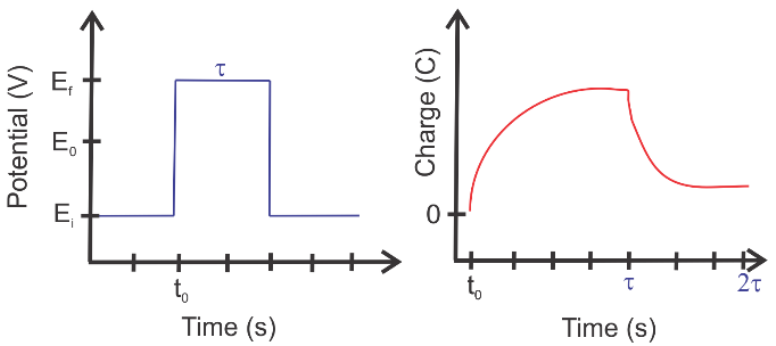

Figure 2.10. (A) Chronocoulometric response for a DNA/MCH self-assembled monolayer without RuHex $\left(Q_{d l}\right)$ and with RuHex $\left(Q_{\text {total }}\right)$. The dash lines represent the fit to the data used to determine the intercept at $\mathrm{t}=0$. (B) Potential and charge in the function of the time for a chronocoulometry measurement. 


\section{CHAPTER 3}

\section{Unraveling the binding mode of a methamphetamine aptamer: a spectroscopic and calorimetric investigation}

\subsection{Abstract}

Nucleic acid aptamers are bio-molecular recognition agents that bind to their targets with high specificity and affinity, and hold promise in a range of biosensor and therapeutic applications. In the case of small molecule targets, their small size and limited number of functional groups constitute challenges for their detection by aptamer-based biosensors because bio-recognition events may both be weak and produce poorly transduced signals. The binding affinity is principally used to characterize aptamer-ligand interactions; however a structural understanding of bio-recognition is arguably more valuable in order to design a strong response in biosensor applications. Using a combination of nuclear magnetic resonance, circular dichroism, and isothermal titration calorimetry, we propose a binding model for a new methamphetamine aptamer and determine the main interactions driving complex formation. These measurements reveal only modest structural changes to the aptamer upon binding and are consistent with a conformational selection binding model. The aptamer-methamphetamine complex formation was observed to be entropically driven, apparently involving hydrophobic and electrostatic interactions. Taken together, our results establish a means of elucidating small molecule-aptamer binding interactions, which may be decisive in the development of 
aptasensors and therapeutics, and may contribute to a deeper understanding of interactions driving aptamer selection. 


\subsection{Introduction}

Nucleic acid aptamers are functional bio-molecular recognition agents that bind to their targets with high specificity and affinity. ${ }^{161}$ They mostly originate from in vitro selection experiments involving the systematic evolution of ligands by exponential enrichment (SELEX) method which, starting from random sequence libraries, optimizes the nucleic acid sequences for highaffinity binding to the presented target ligands. ${ }^{18,19}$ Aptamers have proven to be highly stable, readily adaptable to chemical modification, and exhibit reversible binding. As a consequence, aptamer-based biosensors (aptasensors) are likely to replace antibody-based biosensors. ${ }^{15,17}$ An understanding of the aptamer-ligand binding mechanism is valuable for sensing applications. With this knowledge, the design of the specific binding pocket by truncation or addition of nucleotides, or hybridization with a complementary nucleotide sequence can result in enhancement of the specificity and the signal transduction, and thus the sensitivity of the sensor. ${ }^{115,}$ 162-164 In addition, the most appropriate transduction system for aptasensors can be selected according to the thermodynamic and structural information derived from the aptamerligand complex formation. ${ }^{98,99}$

The binding dynamics of aptamer-ligand systems can be described by three binding models, similar to protein-ligand interactions within the context of folding tunnels and free energy basins (Figure 3.1). ${ }^{35}, 49,162,165$ The lock-and-key (LAK) binding model (Figure 3.1A) simplifies the binding interaction as between a rigid bio-receptor and ligand. ${ }^{47}$ Consequently, their binding interfaces are assumed to be perfectly matched. Accounting for this assumption in the case of aptamer-ligand interactions, the specific nucleotide strand is modeled as though it undergoes no structural change upon ligand addition. $\pi$-Stacking, van der Waals interactions, and hydrogen bonding between the aptamer and the ligand stabilize the complex formed. ${ }^{166}$ The induced-fit (IF) binding model (Figure 3.1B) considers the aptamer to have a flexible binding site able to adopt the optimal binding conformation (or structural 'switch') for a ligand. ${ }^{50}$ Therefore this model does not require the initial binding interfaces between the aptamer and ligand to match. The structural switch causes the formation of Watson-Crick or non-Watson-Crick base pairings in the aptamer structure, which enables the arrangement of a specific binding interface for the ligand. In addition to the aptamer-target interactions considered in the LAK binding model, the formation of new intra-aptamer hydrogen bonds is possible. $^{35}$ 
The conformational selection (CS) binding model (Figure 3.1C) acknowledges the inherent dynamic behavior of the aptamer in solution. Numerous conformational states coexist in equilibrium with different population distributions; the ligand can bind selectively to one of the conformational states, ultimately shifting the equilibrium towards this state. ${ }^{167}$ The aptamer-ligand interactions for the CS and LAK binding models are similar: all aptamer conformations in the solution are assumed to be formed before the target addition and no additional intra-aptamer hydrogen bondings occur.

\section{(A) Lock-And-Key}
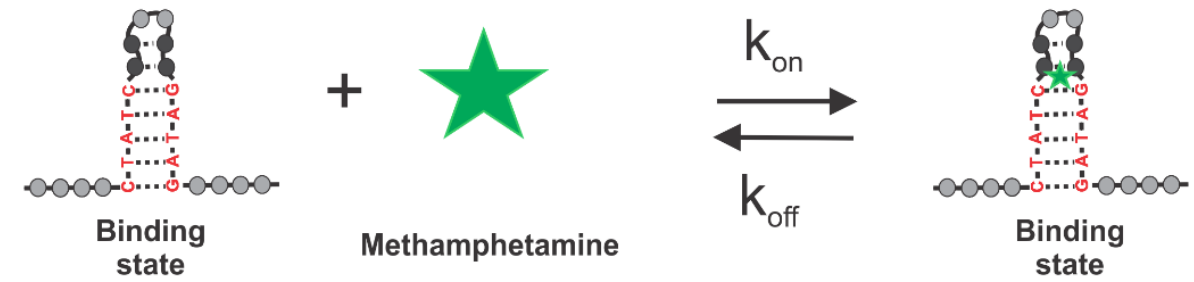

(B) Conformational-Selection
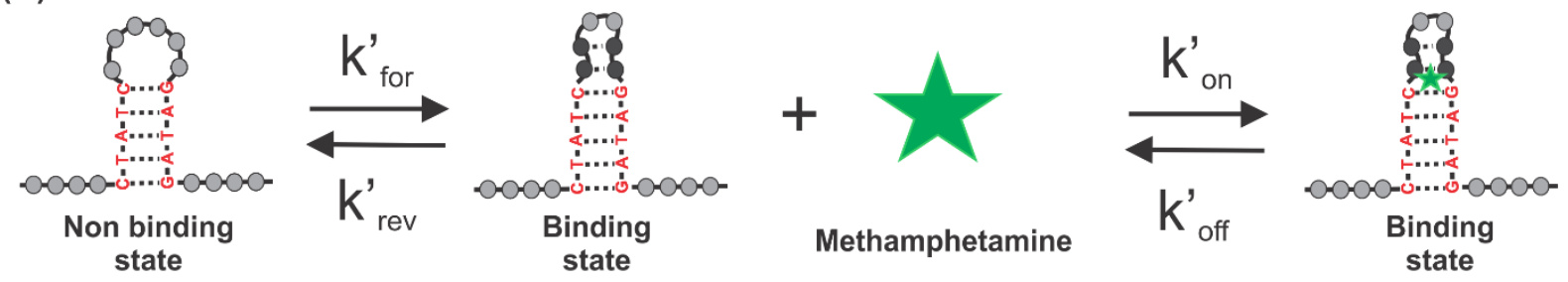

(C) Induced-Fit
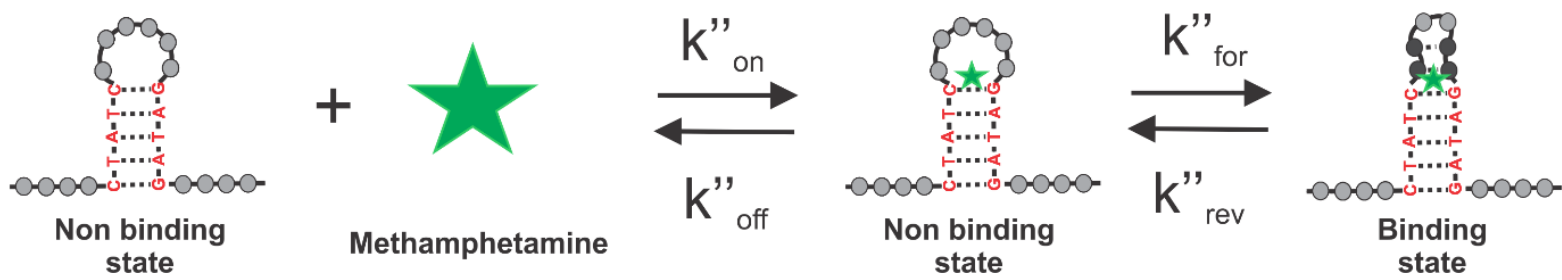

Figure 3.1. Binding models of bioreceptors in the context of aptamer-ligand binding. (A) Lock and Key (LAK), (B) Conformational Selection (CS) and (C) Induced Fit binding models; representation relates to the interaction of the Aptamer-2-40mer with methamphetamine. Binding constant equations are found in the section of Figure 1.5.

In reality, aptamers are known to retain structural plasticity, and binds to their targets by changing their structure in accordance with the target structures. ${ }^{166}$ In seeking to develop aptamers for small-molecules sensing applications, a significant and measurable biorecognition event is required. In this context, it is important to know whether the aptamer-ligand interaction is necessarily accompanied by a structural change. If so, the timing of the conformational change in relation to the binding event must be determined. Furthermore, small-molecule 
ligands, by virtues of negligible size and few functional groups may not induce a significant structural change. Finally, the focus on the dissociation constant, $K_{D}$, for sensing experiments remains valuable, but the crucial requirement for detection by sensors is the measurable transduction of the bio-recognition event. Without specific and significant structural change, a reliable signal may not be detected.

Here, we propose an aptamer characterization workflow using spectroscopic and calorimetric methods coupled with in silico predictions to get a more complete understanding of the interaction between an aptamer and its small molecule ligand. This workflow starts with the determination of the overall complex tertiary structure parameters and concludes with an indepth analysis of the specific region and binding interactions between the aptamer and the ligand. Altogether, this information allows us to propose a detailed binding mechanism for the interaction between a small molecule and a SELEX-generated aptamer.

Methamphetamine (Meth) is a controlled substance that produces a psychological and physical stimulant effect and can lead to dependency. The simple structure of Meth makes it relatively easy to produce illegally. A dwelling can become contaminated with Meth residues if the drug is manufactured or even smoked within. ${ }^{123}$ An aptasensor for detection and quantification of Meth in building environments is the proposed application of the aptamer studied here.

The combination of nuclear magnetic resonance, circular dichroism, and isothermal titration calorimetry experiment allows us to propose a binding model for the new Meth aptamer and determine the main interactions during complex formation. Little structural change in the aptamer was detected, and a conformational-selection binding model was proposed. Entropically driven formation of the aptamer-Meth complex, combining hydrophobic and electrostatic interactions, was observed. Molecular docking simulations support the findings made with NMR and ITC.

This approach is applicable to any aptamer selected for binding a small molecule, enabling a better structural understanding of the complex formed. In the context of aptasensor development, the structural information collected will help in the design and choice of the most suitable transduction platform. 


\subsection{Results and discussions}

The 75 nucleotides long methamphetamine (Meth) aptamer family developed via Affinity Matrix SELEX comprised four structures: Aptamer-1, Aptamer-2, Aptamer-3, and Aptamer-4 (Table A.1) At pH 7.5, the cationic form of Meth dominates (Figure 3.2), making the ammonium part of the structure hydrophilic and electron deficient (red shading), while the phenylpropyl moiety is hydrophobic and electron-rich (grey shading). The hypothetical interactions with the DNA strand of the aptamer will be electrostatic attractions with the cationic nitrogen, hydrogen bond formation between the protonated amine and an atom of oxygen or nitrogen in the aptamer, and hydrophobic and $\pi$-stacking with the phenyly ring. ${ }^{166 \text {, }}$ 168

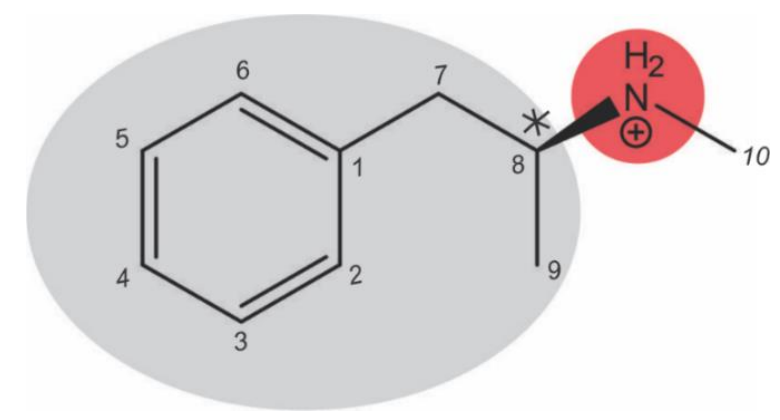

Figure 3.2. Skeletal formula of protonated Meth, as dominant at $\mathrm{pH} 7.5$.

\section{Fluorescence assay reveals binding affinity trends among the Meth aptamer family.}

To compare the binding affinity of the four aptamers with Meth and to guide the choice between the four aptamers for further structural investigations, a fluorescence experiment was conducted. This assay is based on the ability of the SG fluorescence dye to intercalate with partially double-stranded DNA (pdsDNA). ${ }^{169,170}$ A fluorescence emission only occurs when SG is intercalated into pdsDNA; when the SG is displaced from the DNA strand the fluorescence emission ceases. 
Five different samples of each aptamer-SG complex at a given concentration with increasing Meth concentrations were monitored by fluorescence spectroscopy. A proportional decrease of fluorescence intensity at $520 \mathrm{~nm}$, with increasing Meth concentration, is monitored for each aptamer (Figure 3.3A). This fluorescence decrease is produced by the competition between the Meth and the SG molecule to interact with the DNA strand or it is produced by the release of the SG molecule due to an aptamer structural change. ${ }^{169,171}$
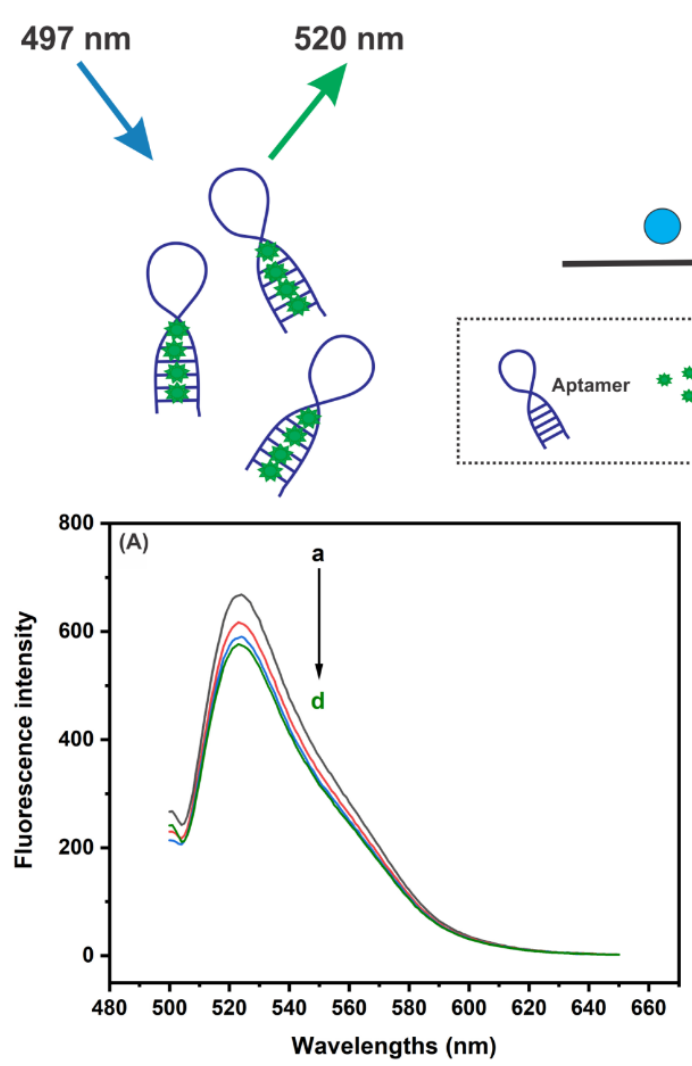
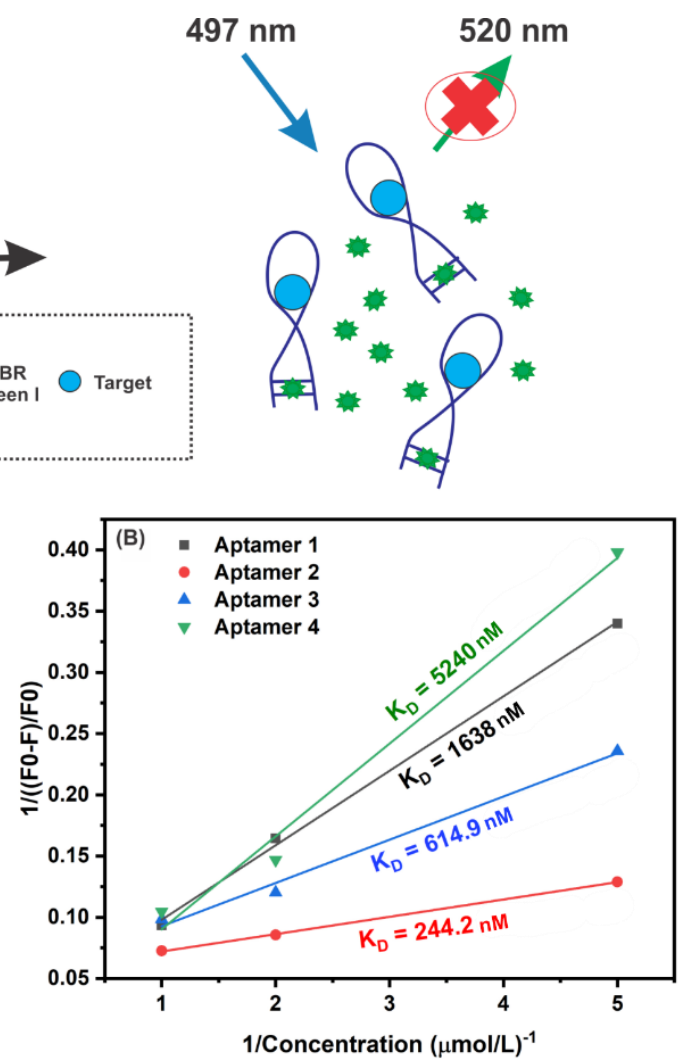

Figure 3.3. Meth titration ( $0 \mu \mathrm{M}$ (a), $0.2 \mu \mathrm{M}$ (b), $0.5 \mu \mathrm{M}$ (c) and $1 \mu \mathrm{M}$ (d)) for the Meth aptamers family. (A) A fluorescence decrease at $520 \mathrm{~nm}$ is monitored for Aptamer-2. (B) Linearization of the Langmuir isotherm for each aptamer.

The normalized fluorescence raw data plotted in function of the Meth concentration gives the known Langmuir behavior. Therefore, a Langmuir isotherm has been drawn for each aptamer. ${ }^{172}$ The equation of the Langmuir isotherm, related to a fluorescence experiment where the $[$ Meth] is greater than the [Aptamer], is the following:

$$
\frac{F_{0}-F}{F_{0}}=B \max \times \frac{[\text { Meth }]}{[\text { Meth }]+K_{D}}
$$


where, $F_{0}$ is the initial fluorescence of the Aptamer-SG complex without Meth, $F$ represents the fluorescence of the Aptamer-SG-Meth complex at one Meth concentration, Bmax is the isotherm constant, [Meth] represents the concentration of Meth and $K_{D}$ is the dissociation constant. Based on the linearization of equation (1), $K_{D}$ values were calculated and represented in Figure 3.3B. Aptamer-2 shows us the greatest binding affinity trend upon Meth addition for the SG assay.

\section{Circular Dichroism (CD) shows that Meth aptamer family, in the presence or absence of Meth, have a B-form structure}

CD spectra (Figure 3.4) revealed the duplex structure of the aptamers in their most stable forms at the conditions used for the experiment. ${ }^{136,173}$ The positive ellipticity peak at $277 \mathrm{~nm}$ and the negative ellipticity peak at $249 \mathrm{~nm}$ are characteristic of a B-form structure. ${ }^{23,129,174}$ Heterogeneous nucleotide DNA sequences adopt this structure in contrast to RNA strands that adopt an A-form structure (positive band at $260 \mathrm{~nm}$ and negative band at $210 \mathrm{~nm}$ ). Another example of a specific duplex structure is the rich guanine sequences that will adopt preferentially G-quadruplex structures (two positive bands at 215 and $295 \mathrm{~nm}$, negative band at $260 \mathrm{~nm}) .^{41}$ 
In our case, the addition of Meth to the four aptamers gave no shift nor considerable changes in peak intensity for the two distinguishable B form peaks (Figure 3.4). This indicates that no significant conformational change occurs for each aptamer upon target addition, and so the aptamers retain their B-forms conformation upon target addition. Regarding the binding models, this could indicate that the major secondary structural motif is preformed and is not dependent on the presence of the target, meaning the IF binding model could be ruled out.
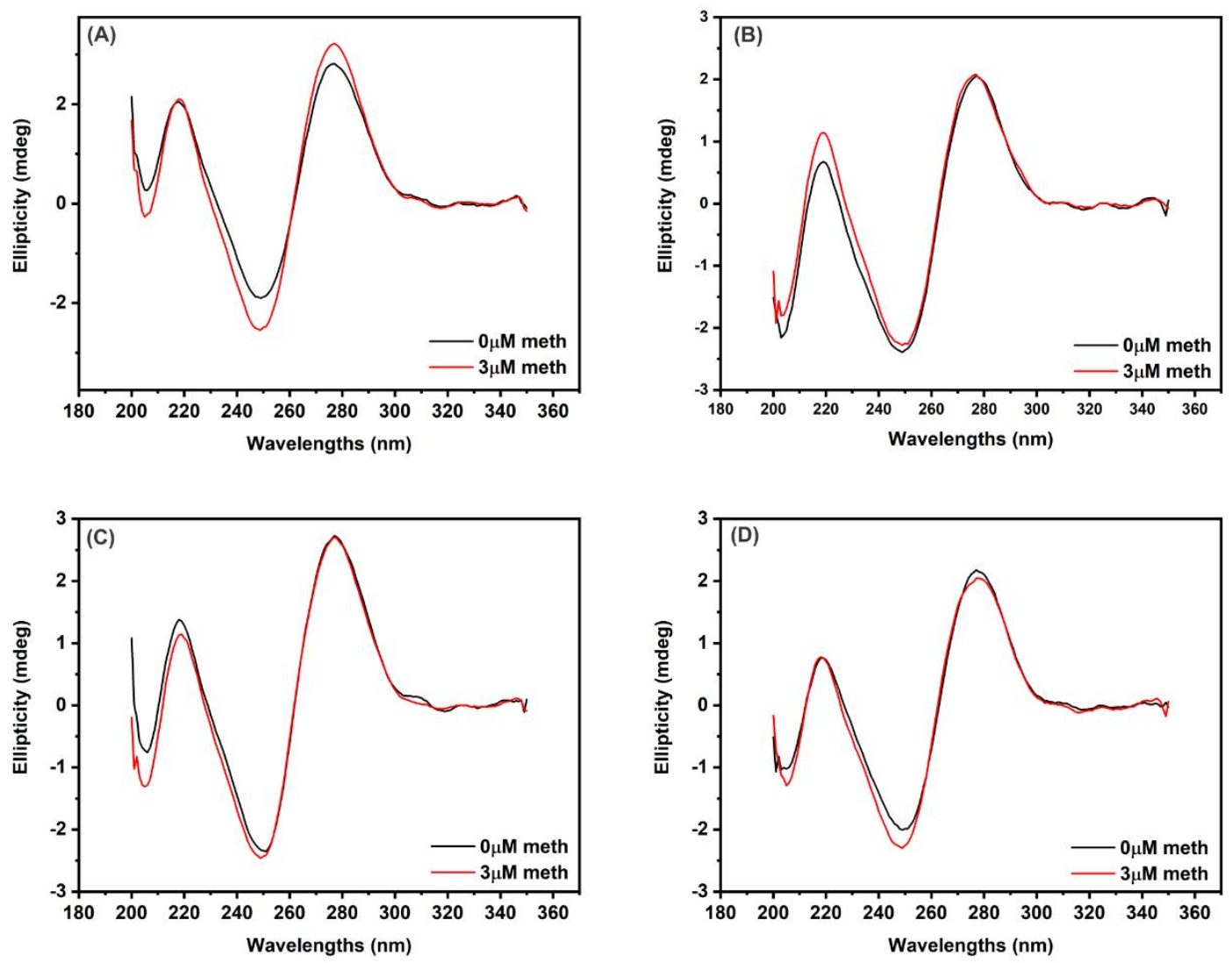

Figure 3.4. CD experiments for (A) Aptamer-1 (B) Aptamer-2 (C) Aptamer-3 and (D) Aptamer-4 upon Meth addition. The positive ellipticity peak at $277 \mathrm{~nm}$ and the negative ellipticity peak at $249 \mathrm{~nm}$ are characteristic of a B-form structure.

Based on the best $K_{D}$ with the SG assay $\left(K_{D}=244.2 \mathrm{nM}\right.$ in Figure 3.3) and on the structural information acquired with CD experiments (Figure 3.4), Aptamer-2 was chosen for a more thorough structural investigation. 


\section{Computational predictions disclose a potential secondary structure of the truncated Meth aptamer, Aptamer-2-40mer}

To obtain simplified NMR spectra for analysis, a truncated aptamer variant (reduced from 75 to 40 nucleotides) was used: Aptamer-2-40mer. The non-essential parts (5' primer and $3^{\prime}$ primer) of the original Aptamer-2 were removed. The primers are necessary to amplify targetbound sequences by PCR during the SELEX process. They do not belong to the randomized part of the aptamer and thus can be removed without significant effect on ligand binding. ${ }^{175}$ Additionally, they can convolute results by interacting non-specifically with the ligand and so their removal is desirable for our subsequent studies.

For Aptamer-2-40mer, three secondary structures were predicted computationally by Mfold and RNAstructure. ${ }^{176,177}$ All three structures contained a hairpin fold with duplex region (Figure 3.5, hairpin circled). The form represented in Figure 3.5 was calculated by Mfold to have the lowest Gibbs free energy at $20{ }^{\circ} \mathrm{C}$ and at the specific buffer ionic strength used. Moreover, the structure shown in Figure 3.5 is consistent with that predicted by Mfold for the original 75mer version of the Aptamer-2. NMR analysis was undertaken to confirm the presence of the hairpin loop structure.

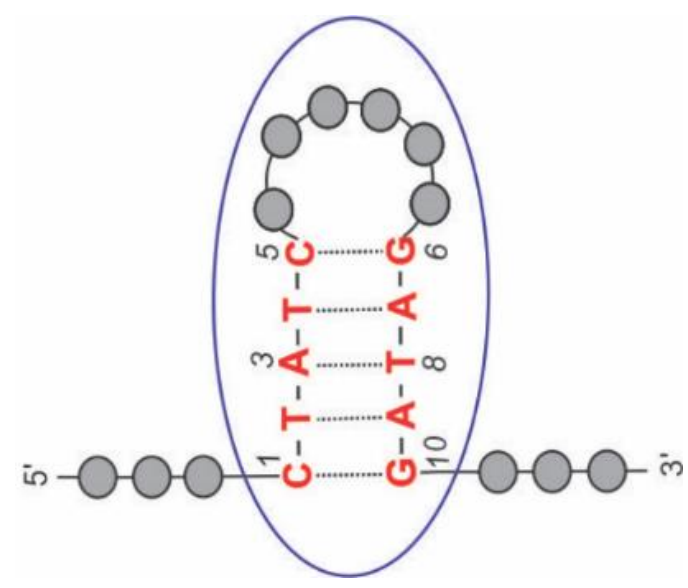

Figure 3.5. Aptamer-2-40mer representation with the hairpin structure encircled.

\section{NMR experiments with Aptamer-2-40mer partially identify the nucleotides belonging to the stem}

The ${ }^{1} \mathrm{H}$ NMR spectrum of Aptamer-2-40mer (Figure A.1) is typical of a partially doublestranded DNA strand. ${ }^{178}$ Peaks observed in the 9-15 ppm region correspond to imino protons in the thymine and guanine bases. ${ }^{141,142}$ Due to proton exchange between imino protons and 
bulk solvent, the only imino protons typically observed are those involved in hydrogen bonding (H-bonding) through nucleotide base pairing, which can include Watson-Crick (Figure 3.6) or non-Watson-Crick interactions, or otherwise protected by structural folding. ${ }^{141,142,179}$ These types of interactions influence the tertiary structure that may contain a solvent-exposed pocket capable of binding proteins and small molecules. ${ }^{180,181}$ The resonance region of the WatsonCrick imino protons in an ${ }^{1} \mathrm{H}$ NMR spectrum is $12-15 \mathrm{ppm}$ and the resonance region of nonWatson-Crick imino protons is $9-12$ ppm. ${ }^{141,142}$
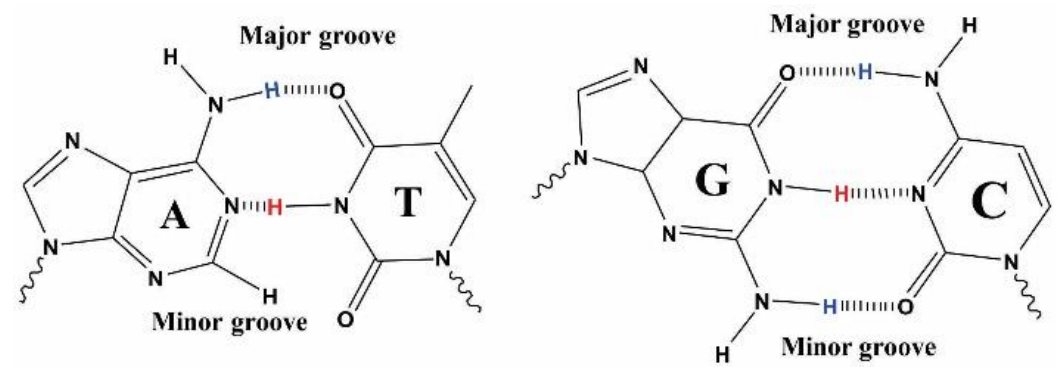

Figure 3.6. Watson-Crick base pairs A-T and G-C.

Aptamer-2-40mer contains five Watson-Crick base pair interactions (two G-C and three A-T) according to the predicted Mfold structure (Figure 3.5). Only thymine and guanine imino protons involved in this hydrogen bonding are expected to be visible in the ${ }^{1} \mathrm{H}$ NMR spectrum (Figure 3.6). The three peaks observed between 13.2 and $13.8 \mathrm{ppm}$ (Figure 3.7A) correspond closely in chemical shift to those observed previously for thymine imino protons ${ }^{111}$ and could therefore represent the three thymine imino protons involved in Watson-Crick hydrogen bindings in Aptamer-2-40mer. ${ }^{142}$ Furthermore, NOE correlations were observed between resonances 13.37 and 7.30 ppm, 13.49 and 7.82 ppm, and 13.54 and 7.26 ppm (Figure 3.7B). These are proposed to correspond to through-space interactions between the thymine $\mathrm{N} 3 H$ and the adenine $\mathrm{C} 2 \mathrm{H}$ which, in Watson-Crick base pairing, is known to be significant. ${ }^{141,182}$ Therefore, the ${ }^{1} \mathrm{H}$ NMR and NOE results are consistent with the A-T base pairs proposed by Mfold to be present in the Aptamer-2-40mer stem in the favored structural form (Figure 3.5). Due to the similar chemical environment of thymines $\mathrm{T} 2$ and $\mathrm{T} 4$, their imino chemical shifts are expected to be very similar. By deduction, the two imino protons observed at 13.49 and $13.54 \mathrm{ppm}$ are tentatively assigned as those of thymines T2 and T4, and, at $13.37 \mathrm{ppm}$, the imino proton of thymine $\mathrm{T} 8$.

The two G-C pairs in the structure predicted by Mfold are not clearly visible in the ${ }^{1} \mathrm{H}$ NMR spectrum. This may be due to their position at the ends of the stem, meaning they are more 
prone to solvent exchange than the three NH protons of the thymines positioned in the middle of the stem. A weak resonance observed at $12.97 \mathrm{ppm}$ may represent one imino $\mathrm{NH}$ from a guanine in the stem. This cannot be confirmed by NOE correlations in G-C base pairs due to the lack of a formamidine proton (or other similarly sited proton) in cytosine. ${ }^{141}$

The other chemical groups involved in hydrogen bonding are the amino groups of adenine, guanine, and cytosine (Figure 3.6), and these are observed in the 7.5-8.5 ppm region (Figure S4). ${ }^{141,142}$ This NMR-based structural assignment partially confirms the secondary structure predicted by Mfold in that the stem composition of three adenine-thymine Watson-Crick base pairs correspond to the spectral observations.
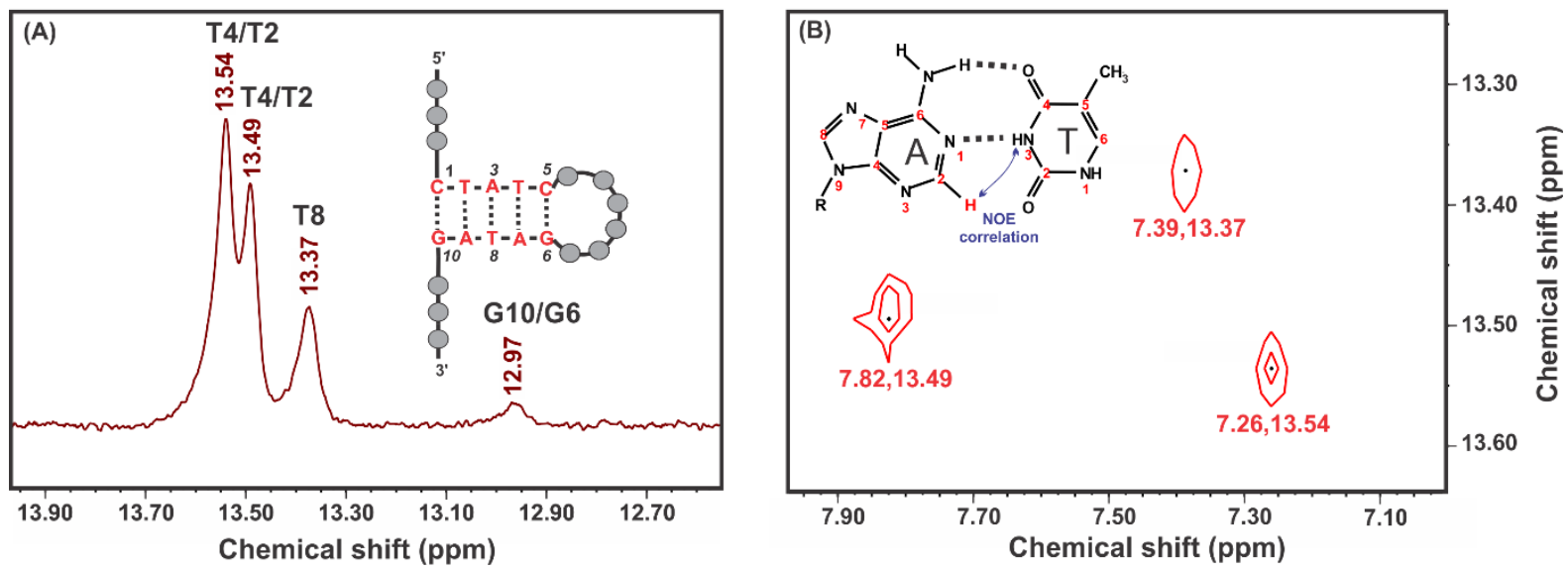

Figure 3.7. (A) Expansion of ${ }^{1} \mathrm{H}$ NMR spectrum of Aptamer-2-40mer showing three thymines imino protons peaks (B) 2D NOESY correlations between the three imino protons and the three adenine C2 protons showing A-T base pairing.

\section{${ }^{1} \mathrm{H}$ NMR spectroscopy reveals new imino proton signals upon Meth binding to Aptamer-2-40mer}

${ }^{1} \mathrm{H}$ NMR spectroscopy was used to investigate the binding mechanism and elucidate which part of Aptamer-2-40mer is involved in target recognition.

A comparison of the ${ }^{1} \mathrm{H}$ NMR spectra of Aptamer-2-40mer in the absence and presence of Meth (saturated, 100\%) shows the formation of four new imino resonances (peaks 5, 6, 7 and 8 in Figure 3.8) in the chemical shift region of hydrogen bonding for non-Watson-Crick base pairs. ${ }^{142}$ This is in line with studies by Neves et al. ${ }^{29}$ and Jenison et al. ${ }^{1}$ in which ligand addition caused additional resonances to appear, indicating that binding of Meth either induces a conformational change in the aptamer or stabilizes a Meth-bound aptamer conformation that otherwise exists in dynamic equilibrium with other unbound aptamer conformations. 
Upon addition of 1 molar equivalent Meth, there was an apparent reduction in the intensity of peak 1 (assigned to the thymine imino proton involved in A-T2 or A-T4) relative to the other A-T resonances. This could be interpreted as a weakening of one of the two A-T interactions in the stem caused by Meth addition.

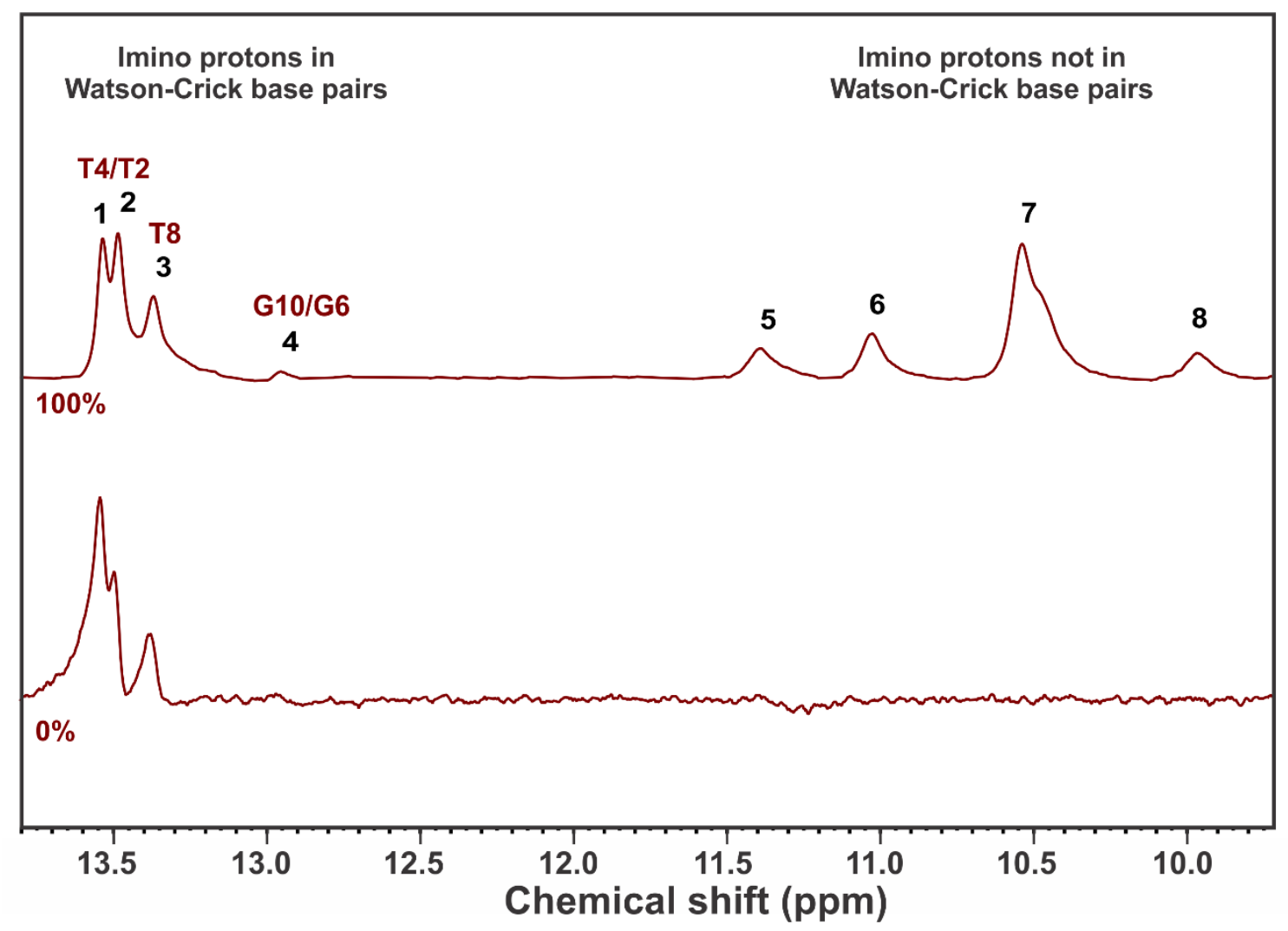

Figure 3.8. ${ }^{1} \mathrm{H}$ NMR spectra of Aptamer 2-40mer with molar loadings of Meth $0 \%$ and $100 \%$.

The appearance of the peaks 5, 6, 7 and 8 (Figure 3.8), residing in the resonance region of imino protons not involved in Watson-Crick base pairs, leads to several hypotheses (vide infra). Isothermal titration calorimetry is used, as described in the next section, to provide thermodynamics parameter to enable a better understanding about the creation of these new peaks and to propose a binding model related to the Meth-Aptamer-2-40mer complex formation.

\section{Isothermal titration calorimetry (ITC) confirms the stem importance and unveils an entropically driven binding reaction}

ITC was used for collecting thermodynamic properties and binding affinities related to Aptamer-2-40mer - Meth binding. Comparison of the original Aptamer-2-40mer with a 
mutated Aptamer-2-40mer sequence (Table A.1), where the base-paired stem is not able to form, highlights the importance of the stem and the resulting loop for binding. As shown in Figure 3.9A, the reaction is endothermic (heat consumption upon target titration) for the Aptamer-2-40mer, whereas the mutant lacking the ability to form a stem shows no variation of the calorimetric signal upon target addition in Figure 3.9B.
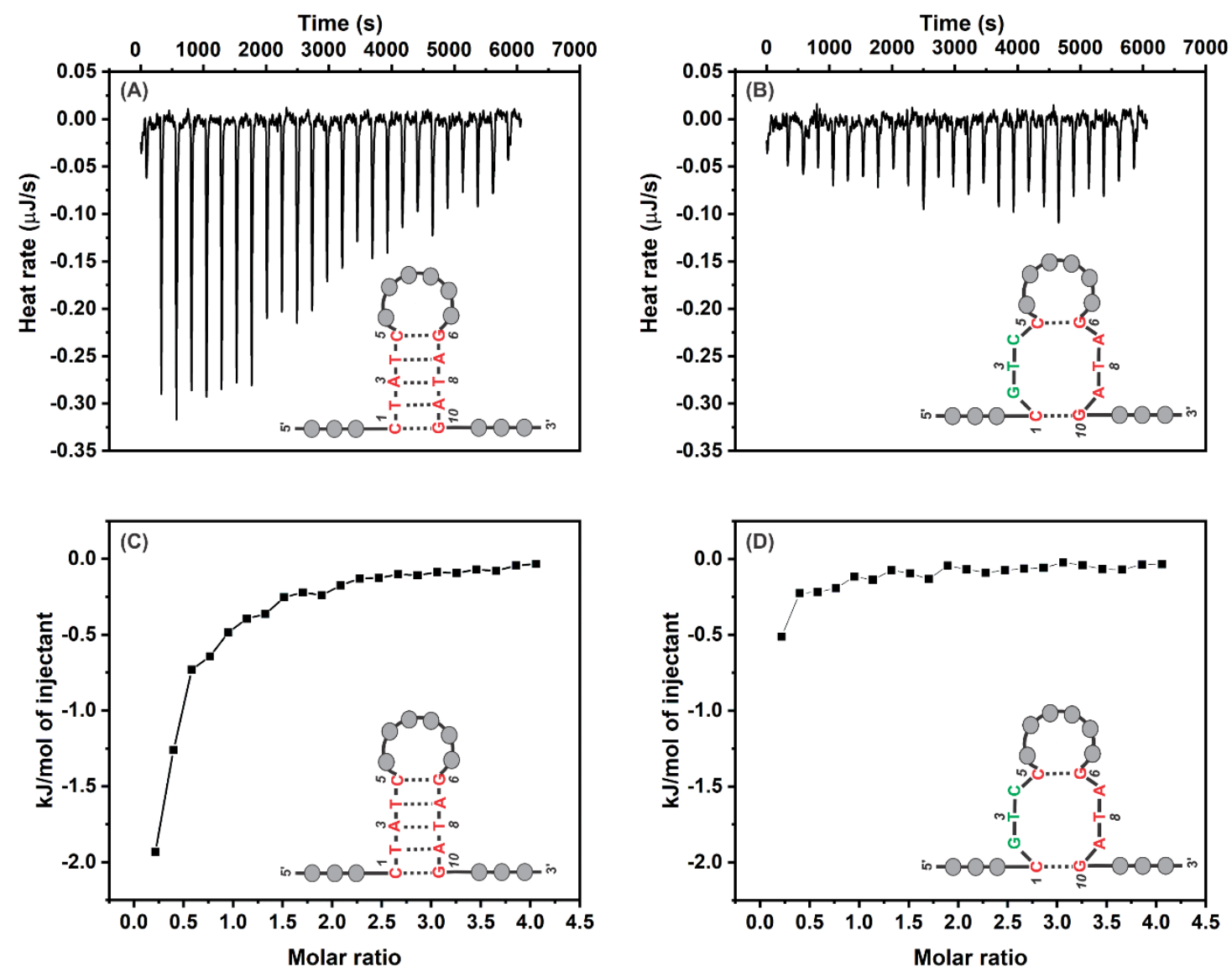

Figure 3.9. ITC results for the Aptamer-2-40mer and the mutated Aptamer-2-40mer. The heat profiles for (A) Aptamer-2-40mer and (B) mutated Aptamer-2-40mer are represented. The integrated heat profiles are represented for (C) Aptamer-2-40mer and (D) mutated Aptamer-2-40mer. The heat profiles presented have been corrected for the heat of dilution of the titrant and for the heat of dilution of the aptamer.

The mutated Aptamer-2-40mer was unable to bind with Meth (Figure 3.9B and Figure 3.9D), which was attributable to the fact that the two remaining Watson-Crick base pairs cannot stabilize the stem formed in Aptamer-2-40mer. This experiment shows that disrupting the stem 
led to a full loss of binding, allowing us to conclude the stem is important to the specific binding event.

The enthalpic signal is weak and slightly endothermic (unfavorable contribution to the overall free energy of the aptamer-Meth complex), indicating disruption of non-covalent bonds (hydrogen bonds between the surrounding water and the aptamer surface) during the interaction between the ligand and the aptamer. ${ }^{35}$ Insufficient new hydrogen bonding interactions are created to overcome the enthalpy penalty. The weak calorimetric signal makes it hard to reliably extract $K_{D}$ and $\Delta S$ but in the absence of strong favorable enthalpic term, binding between Aptamer-2-40mer and Meth must be entropically driven.

The entropic component makes a favorable contribution to the overall free energy of the aptamer-Meth complex. The dominant contributors to the entropic effect could be the release of ordered solvated water molecules and counter-ions during ligand binding. ${ }^{52,183}$ A large favorable entropy component has previously been observed in hydrophobic binding interactions. ${ }^{35,39,137,184}$ Indeed, when ligand binding is associated with a large water gain entropy, the energy decrease (related to the attractive interactions between the aptamer and the Meth) is canceled out by the energy increase originating from the energetic dehydration effect upon binding. ${ }^{52}$ Given the complementary charges of the cationic Meth ligand and the anionic phosphate backbone of Aptamer-2-40mer, we can suggest that an electrostatic attraction may help to capture Meth molecules and position them near the stem region where hydrophobic interactions can be manifest. A related thermodynamic model (unfavorable binding enthalpy with a favorable binding entropy) has been previously proposed for the adsorption of the anionic surface of cytochrome b5 on a positive charged anion-exchange chromatography. ${ }^{185} \mathrm{In}$ both cases, an unfavorable overall binding enthalpy is compensated by a strong entropic contribution, and electrostatic interactions influence the nature of binding.

\section{Combination of spectroscopic and calorimetric data to establish a conformational selection binding model}

By linking the results obtained in CD, NMR, and ITC, a binding model can be used to explain the Aptamer-2-40mer interaction with Meth. The three main binding models related to a bioreceptor - ligand interaction were displayed in Figure 3.1.

From the ${ }^{1} \mathrm{H}$ NMR experiment, the creation of four imino proton peaks not involved in WatsonCrick base pairs upon Meth addition was observed (peaks 5, 6, 7 and 8 in Figure 3.8). The 
formation of the new peaks in the ${ }^{1} \mathrm{H}$ NMR spectrum could result from intra-aptamer $\mathrm{H}$ bonding (consistent with IF or CS binding models) or from other imino protons resonances such as H-bond of Meth-Aptamer, NH of Aptamer only or $\mathrm{NH}_{2}$ of Meth only (IF, CS and LAK binding model). Two scenarios for the formation of these peaks can be considered:

[1] Meth is a hydrogen bond donor (Figure 3.2) and, thus, one H-bond may be formed between Meth and an oxygen or a nitrogen atom of a nucleotide base. This H-bond may have a chemical shift in the region of 10-11 ppm, which is the region of the imino protons not involved in Watson-Crick base pairs. In the case of a theophylline aptamer, Zimmermann et al. 186 observed the formation of a pseudo-base pair between the cytosine and the purine-like theophylline in the appearance of an H-bonding resonance in the region of $15 \mathrm{ppm}$. The Meth $\mathrm{H}$-bonding interaction with an aptamer nucleotide will resonate in a more upfield region due to the lower electronegative environment provided by the Meth molecule. In addition, one of the Watson-Crick base pairs from the stem might be disrupted upon Meth addition and, therefore, one of the imino protons remains free in solution. It is still structurally protected by the stem from solvent exchange effects; thus, it is visible in the ${ }^{1} \mathrm{H}$ NMR spectrum and resonates in the non-Watson-Crick region. Two signals are expected to appear in the 10.0-11.5 ppm region.

[2] No H-bond is formed between the Meth and the aptamer; however, the amine group can be resolved because Meth may interact with the aptamer in a specific cavity where the protonated amine group of the Meth is protected from solvent exchange. The cationic amine group of Meth has two hydrogens that are not visible in the ${ }^{1} \mathrm{H}$ NMR spectrum of the unbound ligand due to proton exchange with the aqueous environment but may become visible in the bound form (Figure A.2). Neves et al. ${ }^{111}$ showed that, when bound with its aptamer, the cocaine imino proton became visible in the $10 \mathrm{ppm}$ region of the ${ }^{1} \mathrm{H}$ NMR spectrum. A similar effect may also occur with the Meth amino protons. Indeed, if Meth interacts with a specific cavity of the aptamer, proton exchange with the aqueous environment would similarly decrease due to the structural protection of the aptamer, and possibly resolve the two amino proton resonances. The electron demand is similar for Meth and cocaine, nevertheless, as Meth is a secondary amine and cocaine a tertiary amine the chemical shift will differ slightly. One of the Watson-Crick base pairs from the stem may be disrupted and, therefore, one of the imino protons is free in solution (visible as explained in the first scenario) and is resonated in this region. Three signals in the 10.0-11.5 ppm region are expected to appear.

Neither of these scenarios explain the four new NMR peaks observed. Consequently, some of the four peaks observed may be related to intra-aptamer $\mathrm{H}$ bonds and, thus, it indicates that 
different structures of the aptamer are present in solution before and after target addition. Consequently, the LAK binding model is ruled out. The structural observations obtained with NMR are in line with the CS and IF binding models.

A main difference between the CS and the IF binding models is the number of conformational states present initially in solution $35,49,162,165$. The IF binding model assumes an initial nonbinding conformation present in solution; by adding the target to a 1:1 molar ratio (aptamer:target), it is considered that a new binding state is present and no non-binding conformation remains in solution. From a thermodynamic perspective, the IF binding model would require a strong attractive interaction (i.e. enthalpic) to drive this structural change ${ }^{35}$, 187, 188 . This is inconsistent with the lack of enthalpic attraction observed in the ITC experiment so we can suppose that the IF binding model for the Aptamer-2-40mer - Meth interaction can be ruled out.

Moreover, the $\mathrm{CD}$ experiments support this suggestion because no significant change in the duplex structure upon ligand addition is observed (Figure 3.4). Furthermore, in the ${ }^{1} \mathrm{H}$ NMR experiment, we observed that the three peaks (1, 2 and 3 in Figure 3.7A) related to the stem are present before the target addition. Despite the modest decrease in intensity of peak 1, all are still present after the target addition. No new resonance peaks related to Watson-Crick base pair formation are visible after Meth addition. These two observations provide additional evidence that no major aptamer structural changes occur upon Meth addition.

Finally, the CS (conformational-selection) binding model is considered. Different conformations of the aptamer exist in a dynamic equilibrium in solution as shown in Figure 3.1. ${ }^{35,49,189}$. Meth binds specifically to one of these conformations. At $0 \%$ Meth, the aptamer conformations are in a dynamic equilibrium where $k_{r e v}^{\prime}>k_{f o r}^{\prime}$ (unbound conformation is favored and thus is observed at $0 \%$ Meth). The addition of Meth causes the binding state to be favored in solution $k_{\text {on }}^{\prime}>k_{\text {rev }}^{\prime}$; therefore, the ${ }^{1} \mathrm{H}$ NMR spectrum upon addition of one equivalent (saturated) represents the binding state with additional non-Watson-Crick resonances. Because the loop region contains enough guanine or thymine residues to form non-Watson-Crick base pairs, it is hypothesized that these interactions are situated in the loop region. ITC experiments support this hypothesis as no signal is measured for the mutated Aptamer-2-40mer where the loop is not formed. Furthermore, in support of the thermodynamic data collected, a mixture of binding and non-binding state conformation is present in solution. By adding the target, the equilibrium between the two states shifts to the binding state conformation. Comparatively to the IF binding mode, the equilibrium shift is less significant due to the initial presence of binding state aptamer. Thermodynamically, this equilibrium switch may not afford a strong 
attractive interaction (enthalpic in contrast to the IF binding mode) and, thus, is consistent with an entropically driven binding event.

\section{Molecular modeling supports the experimental NMR and ITC hypotheses}

Following the experimental determination of Meth binding to the Aptamer-2-40mer, molecular docking was used to rationalize the proposed binding hypotheses.

First, a 3D model of the Aptamer-2-40mer was constructed using RNAcomposer. ${ }^{190}$ Mfold ${ }^{176}$ and RNAstructure ${ }^{177}$ provided a $2 \mathrm{D}$ structure corresponding to that observed experimentally. ${ }^{176,177}$ In this study, the stem composed of the three A-T base pairs has been observed and confirmed previously with NMR experiments. Additionally, with the RNAstructure software, the partition function calculation ${ }^{191}$ predicted base pairs probabilities. By using the partition function for the Aptamer-2-40mer, it appears that the five base pairs predicted by Mfold and represented in the Aptamer-2-40mer (Figure 3.5) show a high probability of formation (Figure A.3). Finally, the 2D structure and DNA sequence have been used to generate the 3D structure with RNAcomposer.

Exhaustive docking of Meth revealed several binding models homogeneously distributed within the major groove of the stem region (Figure 3.10). All poses predict the placement of the cationic amine group of Meth near $\mathrm{C} 5, \mathrm{~T} 4, \mathrm{~A}^{\prime}$ (nucleotide from the loop), $\mathrm{T}^{\prime \prime}$ (nucleotide from the loop), $\mathrm{T}^{\prime \prime \prime}$ (nucleotide from the loop), G6, and aromatic ring near T2, A3, and A7. Conformational clustering of the 100 poses gave two distinct clusters; the highest scored was least populated with 12 poses $(\mathbf{C 1})$, whilst the most populated $(\mathbf{C 2})$ contained 88 poses that scored slightly worse (see Figure 3.10B for score distributions). Analysis of $\mathbf{C 1}$ shows the cationic amine of Meth forming a salt bridge with the $\mathrm{T}^{\prime \prime}$ (nucleotide from the loop) phosphate backbone. The remainder of the Meth framework (from methyl-9 to the aromatic ring) resides along a column of polar moieties from the surrounding bases (G6 carbonyl, C5 amine, T4 carbonyl, A7 amine, A3 amine, T8 carbonyl, Figure 3.10C). For $\mathbf{C 2}$, the cationic amine retains the ionic bond with the $\mathrm{T}^{\prime \prime}$ phosphate backbone, but instead, the methyl-9 of Meth is positioned towards the $\mathrm{T}^{\prime \prime \prime}$ thymine methyl group rather than T4 thymine methyl (like in C1, Figure 3.10D). Overall, the docking study suggests Meth could favorably occupy the pocket by forming ionic and hydrophobic interactions with the surrounding environment. These findings are in line with those experimentally deduced, and both highlight the importance of the stem region for Meth binding. The predicted binding modes may also support the hypothesis that 
the evolution of the peak $1{ }^{1} \mathrm{H}$ NMR resonance upon Meth treatment (in Figure 3.8) corresponds to partial disruption of H-bonding between A-T2 or A-T4 base pairs. Furthermore, the hydrophobic interaction and the ionic interaction between the phosphate backbone and Meth correlates with the thermodynamic parameters obtained by ITC (Figure 3.9).

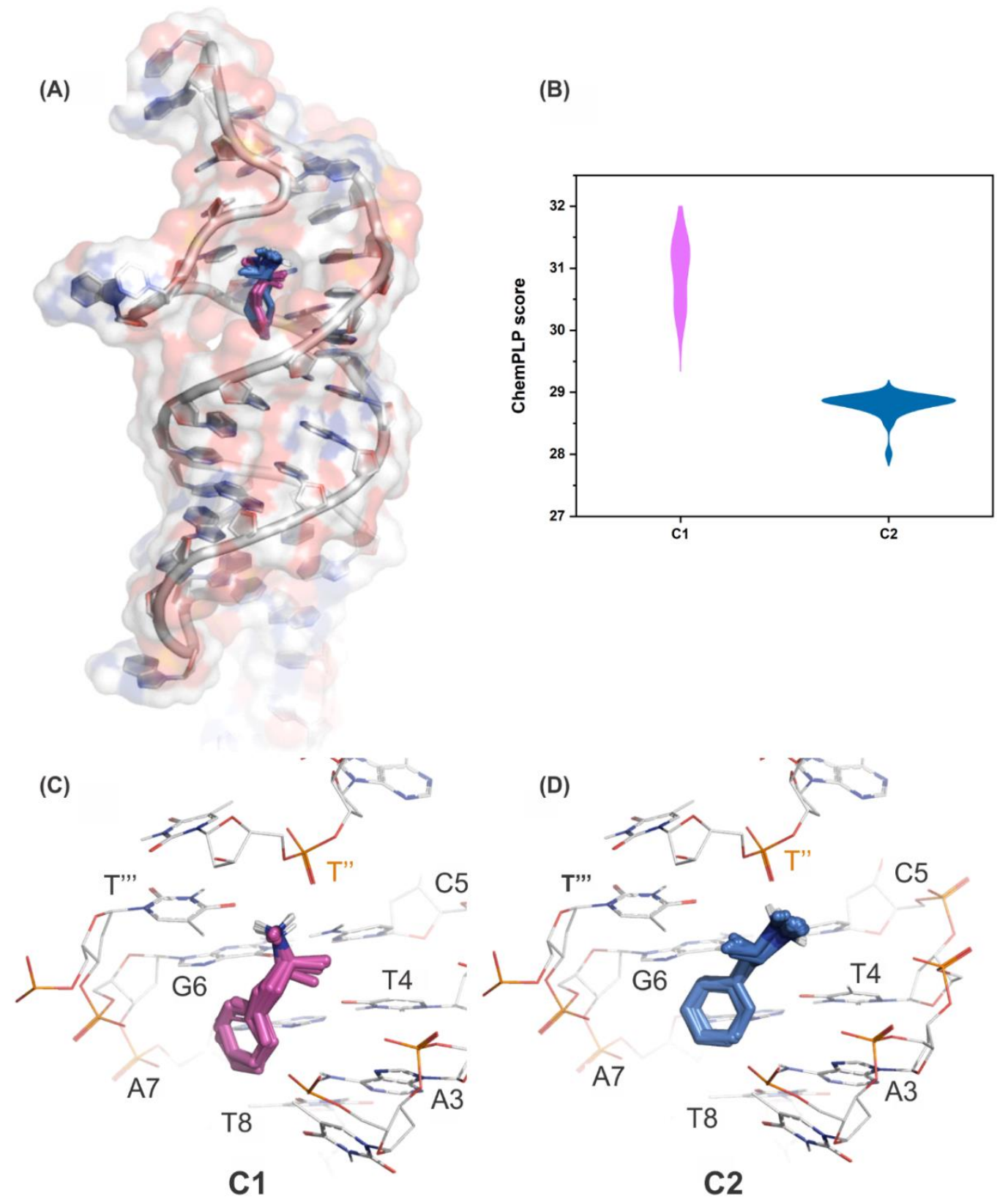

Figure 3.10. Molecular docking of Meth against Aptamer-2-40mer. (A) All 100 docking poses in the hydrophobic pocket are shown as sticks, the aptamer is shown in cartoon form with a transparent surface rendered. (B) A violin plot of the docking scores of each cluster. (C) The 12 poses of cluster_1 in the pocket with surrounding nucleotides shown in wire form. (D) The 88 poses of cluster_2 in the pocket with surrounding nucleotides shown in wire form. Courtesy of Jordan AJ McCone. 


\subsection{Chapter conclusion}

Through a combination of CD, NMR, and ITC experiments, a binding mechanism for the Meth - Aptamer-2-40mer complex is proposed and supported by molecular modeling simulations. Specifically, no significant structural change has been detected with CD experiments upon addition of Meth. NMR experiments indicate a change in the imino region for Aptamer-240mer in solution upon Meth addition that is proposed to consist of a form where new nonWatson-Crick base pairs are present in the loop (potentially arising through a conformationalselection binding process). Moreover, a partial weakening of one of the A-T2 or A-T4 WatsonCrick pairs is tentatively interpreted from the NMR spectra to occur upon target addition. ITC experiments demonstrate the importance of the stem for the binding event. Indeed, by removing the stem, no binding event occurs. Thermodynamic parameters collected with ITC provided information suggesting an entropically driven binding event. Molecular modeling supports the proposed hypotheses in the preferred positions of Meth calculated to be within the major groove of the stem and with interactions to the phosphate backbone of the loop.

This approach is suited to study of other aptamer-small molecule complexes as a characterization protocol prior to development of an aptasensor platform. Following the findings related to the binding region localization as well as the structural switch magnitude of the aptamer upon the target addition, the best transduction strategy needs to be considered. The small magnitude of the conformational switch and accompanying enthalpic change indicate that Affinity Matrix SELEX may not provide the optimal aptamer structures for detection of monofunctional small molecule targets. Other SELEX protocols must be investigated for this purpose.. 


\subsection{Experimental section}

\section{Materials}

AuramerBio (Wellington, New Zealand) selected the 75 nucleotides long Meth aptamers family via "Affinity Matrix Selex". ${ }^{192}$ Synthetic oligonucleotides were obtained from AlphaDNA (Montreal, Canada). DNA strands were purified by SDS-PAGE. The binding washing buffer (BWB) was tris(hydroxymethyl)aminomethane hydrochloride buffer $(2 \mathrm{mM}$ Tris- $\mathrm{HCl}, 10 \mathrm{mM} \mathrm{NaCl}, 0.5 \mathrm{mM} \mathrm{KCl}, 0.2 \mathrm{mM} \mathrm{MgCl}_{2}$ and $0.1 \mathrm{mM} \mathrm{CaCl}_{2} ; \mathrm{pH}$ 7.5). Sodium 3(trimethylsilyl)-1-propanesulfonate (DSS) was used as an internal standard for NMR experiments. ${ }^{193}$ Before any experimentation, the aptamer was denatured at $95{ }^{\circ} \mathrm{C}$ for 5 min followed by cooling in an ice bath for $10 \mathrm{~min}$.

\section{SYBR green assay I}

Each aptamer was mixed in BWB solution with the fluorescence dye SG then a Meth titration was conducted for each of them, a fluorescence signal is monitored along the Meth titration. $10 \mu \mathrm{l}$ of SG $(7 \mathrm{x})$ and $10 \mu \mathrm{l}$ of aptamer $(10 \mu \mathrm{M})$ were mixed. A range of Meth concentrations varying from 0 to $1 \mu \mathrm{M}$ was prepared in BWB and added directly to the SG-aptamer solution to deliver a final volume of $1000 \mu \mathrm{L}$. The fluorescence emission spectra were recorded from 500 to $650 \mathrm{~nm}$ using an excitation wavelength of $497 \mathrm{~nm}$. The emission fluorescence at 520 nm was measured.

\section{CD analysis}

BWB solutions containing each aptamer with and without methamphetamine were analyzed with a $C D$ instrument. $1.5 \mu \mathrm{M}$ solutions of each aptamer were prepared with $3 \mu \mathrm{M}$ of Meth and without Meth in BWB. CD analyses were performed on a Chirascan Spectrometer in a $1 \mathrm{~mm}$ path length quartz cuvette. The spectra were recorded from 380 to $180 \mathrm{~nm}$ at $20^{\circ} \mathrm{C}$ at a rate of $200 \mathrm{~nm} / \mathrm{min}$ and corrected by subtraction of the background scan of the buffer.

\section{${ }^{1} \mathrm{H}$ and Nuclear Overhauser enhancement (NOESY) NMR experiments}

Aptamer sample was analyzed by ${ }^{1} \mathrm{H}$ NMR and 2D NOESY. DNA samples were dissolved in $527 \mu \mathrm{L}$ of BWB containing $10 \% \mathrm{D}_{2} \mathrm{O}$. The final concentration of DNA was $1 \mathrm{mM}$. An internal standard DSS was added to the sample and its final concentration was $1 \mathrm{mM}$. For the presence of Meth, the concentration was fixed to $1 \mathrm{mM}$. NMR experiments were performed on $600 \mathrm{MHz}$ Nuclear Magnetic Resonance Spectrometer type JNM-ECZ600R with data recorded at $20{ }^{\circ} \mathrm{C}$. 
1D proton NMR spectra were acquired with 3500 data points using 1280 scans due to low sample concentration. NOESY spectra were obtained using a mixing time of $200 \mathrm{~ms} .{ }^{194}$ Solvent suppression was achieved by the "Watergate sequence". ${ }^{195}$ Acquired data were processed and analyzed using MestReNova software. ${ }^{196}$

\section{Isothermal titration calorimetry assay}

Calorimetric experiments were achieved for the aptamer and a stem mutated version. Isothermal titration calorimetry (ITC) assays were performed on an Affinity ITC system (TA Instruments Inc., New Castle, DE, USA). $1 \mu \mathrm{L}$ injections of a $2.5 \mathrm{mM}$ Meth solution in BWB were added by a computer-controlled microsyringe at an interval of $240 \mathrm{~s}$ into the DNA aptamer solution $(50 \mu \mathrm{M}$ in BWB, cell volume $200 \mu \mathrm{L})$ with $100 \mathrm{rpm}$ stirring at $20{ }^{\circ} \mathrm{C}$. Heats produced by Meth dilution and aptamer dilution were evaluated and subtracted. The experimental data were fitted to a theoretical titration curve using software supplied by TA, with $\Delta G$ (Gibbs free energy), $K_{D}$ (dissociation constant), and $n$ (number of binding sites per monomer), as adjustable parameters.

\section{Molecular modeling}

Three plausible two-dimensional (2D) structures of Aptamer-2-40mer were generated using Mfold and RNAstructure. ${ }^{176,177}$ The 2D structure, related to the lowest Gibbs free energy, was then imported to RNAComposer ${ }^{190}$ to generate a three-dimensional (3D) model, which was used in subsequent docking studies. The 2D structure of Meth was sketched on Maestro and prepared for docking by generating a low energy 3D conformer using LigPrep. ${ }^{197}$ The program GOLD (Genetic Optimization for Ligand Docking) ${ }^{198}$ was used for all molecular docking analyses. The aptamer binding site was defined as a $100 \AA$ sphere around the coordinates $\mathrm{x}=$ $0.3155 \mathrm{y}=-26.7188 \mathrm{z}=6.1465$. Because GOLD's GA (genetic algorithm) is stochastic in nature, an exhaustive approach was adopted in that $100 \mathrm{GA}$ runs were set per conformer, giving 100 poses for further analysis. Search efficacy was set to $200 \%$ (highest accuracy). The ChemPLP ${ }^{199}$ scoring function was used to score and rank poses, with a higher score corresponding to a pose having a relatively better 'fit' to the cavity. The resulting 100 poses were clustered according to their respective geometries using the cluster conformers.py script implemented in the Schrödinger 2019-2 package. ${ }^{200}$ The average linkage method was used for cluster generation and the optimal number of clusters was automatically determined using the Kelley index. ${ }^{201}$ Finally, clusters were visually compared and merged based on their conformational similarity. Computational modelling has been done by Jordan AJ McCone. 


\section{CHAPTER 4}

\section{Screening the viability of three electrochemical aptasensor approaches}

\subsection{Abstract}

The electrochemical (e-chem) transduction platform used for aptamer-based biosensors (aptasensors) technology is a broad group of transduction systems (previously described in Chapter 1). As a consequence of the inherent high sensitivity and low detection limit of electroanalytical methods; they have attracted considerable attention in the last decade, resulting in many research articles published in this field. Furthermore, electrochemical transduction platforms are simply miniaturized, and therefore, can be used with portable instruments. Finally, these biosensors are cost-effective and provide a fast-analytical response. The growth of electrochemical aptasensors development creates a too exhaustive panel of choice. Moreover, electrochemical aptasensors are restrained to academic laboratories and have not yet proven to be commercially viable. Therefore, no "gold" standard electrochemical aptasensor protocol exists, specifically for small molecule detection.

Here, a screening of popular e-chem aptasensor strategies has been achieved by comparing the time response between a buffer and a meth solution for each system. By using a standardized protocol, the comparison between different e-chem strategies is achievable.

No robust and specific binding signal was observed for each electrochemical method strategy tested. Indeed, all are composed of a significant background drift that is not overcome by the 
specific binding signal. Despite this finding, the electrochemical impedance spectroscopy (EIS) using a thiolated modified aptamer and the ferri/ferrocyanide redox couple appears the most appropriate e-chem aptasensor strategy for the development of a reliable portable methamphetamine detection system. EIS affords a great sensitivity towards the gold surface and is a label-free method. Furthermore, based on the binding model found in Chapter 3, the EIS method prevails as the most suitable for the development of the aptasensor specific to Meth.

Comparing different e-chem strategies between the buffer response and the target response is used as a decision-making method. Optimization and development experiments will be pursued for the EIS approach in Chapters 5 and 6. 


\subsection{Introduction}

As discussed in Chapter 1, electrochemical transduction platforms are preferred to the other transduction systems for methamphetamine (Meth) detection using aptasensor technology. Electrochemical (e-chem) aptamer-based biosensors (aptasensors) offer sensitivity, selectivity, and low cost for detecting a various range of molecules. ${ }^{68,69,202,} 203$ Moreover, e-chem aptasensors provide a broad range of strategies for designing a sensing platform. All take benefit of nanoscale interactions between the target in solution, the bio-recognition layer, and a solid electrode surface.

The electrochemical detection signal monitored is an electrochemical faradaic signal. It is the consequence of an oxidation/reduction reaction that produces electrons. The electron transfer between the redox probe and the working electrode can be electrochemically measured via a current or an impedance value. ${ }^{69}$ The use of a redox probe or a redox couple is essential. That's why the different electrochemical aptasensor strategies can be classified into two main groups based on the redox probe use. ${ }^{68}$

The first category is associated with a redox probe that covalently or non-covalently interacts with the aptamer strand, the electrochemical signal will be monitored with voltammetry. In this category, one redox probe stands out from the redox probes group: the methylene blue (MB) molecule (Figure 4.1). ${ }^{98,204}$<smiles>CN(C)C1=CC2Sc3cc(N(C)C)ccc3NC2C=C1</smiles>

Methylene blue

Leucomethylene blue

Figure 4.1. The oxidation and reduction reaction of the methylene blue molecule.

The non-covalent interactions of the methylene blue molecule with a DNA strand are various as observed in Figure 4.2: (a) the methylene blue molecule is a cationic molecule at pH 7.5. Therefore, an ionic interaction between the negative phosphate group of the DNA strand with the cationic sulfur part of the methylene blue molecule is possible. ${ }^{204}$ 
(b) In partially double-stranded DNA, the pairing of the non-symmetric bases and their stacking gives rise in B-DNA to a small (minor) groove and a large (major) groove. The major groove is characterized by a wider side on the contrary to the minor groove which is characterized by a narrow side. Within each groove, base pairs are exposed and are accessible to interactions with other molecules. ${ }^{26} \mathrm{MB}$ interacts with both grooves because it is a planar molecule, and it has three aromatic rings. ${ }^{205}$

(c) MB is a three aromatic rings planar molecule, so its intercalation via $\pi$-stacking in a partially double-stranded DNA is possible. ${ }^{205}$

(a)

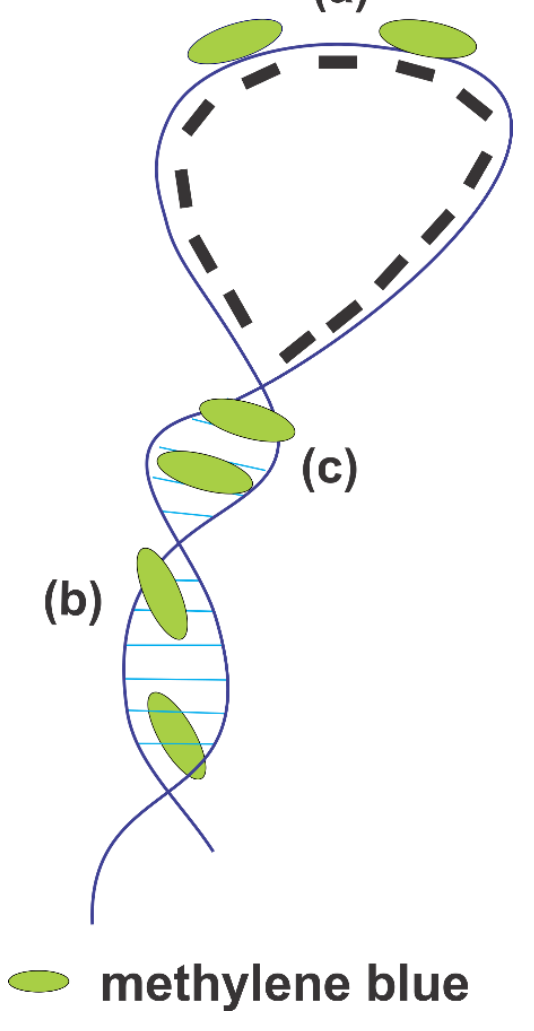

Figure 4.2. Schematic representation of the three non-covalent interactions occurring between the methylene blue (MB) molecule and a partially double-stranded DNA. (a) electrostatic interaction between the negative phosphate backbone of DNA and the cationic part of MB, (b) major and minor groove interactions and, (c) $\pi$-stacking interactions afford intercalations.

Yu et al. ${ }^{206}$ attached a thiolated bisphenol A (BPA) aptamer on a gold electrode. The BPA aptamer is a hairpin structure aptamer. The MB molecule interacts non-covalently with the BPA aptamer by intercalations, electrostatic, and minor/major groove interactions. Upon BPA addition, a structural change of the aptamer occurs, some MB molecules are liberated, resulting 
in the reduction of the electrochemical response. The electrochemical response is monitored via differential pulse voltammetry (DPV). An outstanding linear relationship of detection is presented in the range of $4.38-438000 \mathrm{pmol} / \mathrm{L}$.

In Wang et al., ${ }^{207}$ a partial complementary sequence to the adenosine aptamer is fixed on a gold electrode. Then, the aptamer is partially hybridized with the complementary sequence. MB interacts with the aptamer, and with adenosine addition, the aptamer strand is released from the complementary sequence. A significant decrease of the electrochemical signal that combines the release of the MB non-covalently modified aptamer from the electrode surface and the liberation of the MB molecule due to the adenosine-aptamer specific interaction. Farjami et al. ${ }^{204}$ use a similar protocol for DNA hybridization detection.

Another e-chem aptasensor strategy using MB molecule can be used. MB molecule covalently attached to the aptamer strand is prevalent in the e-chem aptasensor domain. ${ }^{98,99,208}$ The aptamer strand has two ends: the 5'-end and the 3'-end. In e-chem aptasensor systems, one of the two ends can be modified with a thiol group (-SH) which interacts with the gold electrode surface to form a strong bond Au-S. On the other aptamer end, the MB is covalently attached. Baker et al. ${ }^{99}$ detect the cocaine molecule with a MB covalently modified cocaine aptamer attached to a gold electrode. The electrochemical signal related to the reduction of the MB is measured via square wave voltammetry (SWV). A "signal on" system is described, in the absence of target the aptamer is unfolded and the MB probe is away from the electrode surface, a small electrochemical signal is measured. The presence of cocaine induces a structural change of the aptamer to bind to the cocaine molecule. As a consequence, the MB probe comes closer to the electrode surface, and the electrochemical signal increases. A "signal off" system (decrease of the electrochemical signal upon target addition) is described by $\mathrm{Yi}$ et al. ${ }^{98}$ for thrombin detection.

The second category of the e-chem aptasensors uses a redox probe or a redox couple that doesn't associate with the DNA strand, the electrochemical signal is measured with electrochemical impedance spectroscopy (EIS). As the DNA strand and the analyte are not modified with a fluorescent probe, the system is called a label-free system. ${ }^{209}$ The redox couple ferricyanide/ferrocyanide (ferri/ferro) is a standard in the electrochemistry biosensor field (Figure 4.3). ${ }^{210,} 211$ 


\section{$\mathrm{Fe}(\mathrm{CN})_{6}{ }^{3-}+\mathrm{e}^{-} \rightleftharpoons \mathrm{Fe}(\mathrm{CN})_{6}{ }^{4-}$}

Figure 4.3. The oxidation and reduction reaction of the ferricyanide/ferrocyanide couple.

The main advantages of the ferri/ferro couple are the following: it exhibits reversible heterogeneous kinetics, it has stable oxidized and reduced forms, it is soluble in aqueous solution, and, in aptasensors field, it doesn't react or interact with DNA. ${ }^{209,212,213}$ In a standard e-chem aptasensor system using the ferri/ferro couple, the aptamer is attached covalently to the gold electrode via an Au-S bond. Lin et al. ${ }^{214}$ developed a label-free e-chem aptasensor for $17 \beta$-estradiol. By immobilizing a thiol aptamer on a gold electrode and using the ferri/ferro redox couple, they created a monolayer characterized by EIS measurement. Upon addition of $17 \beta$-estradiol, the aptamer produces a structural change to bind specifically to the target and, therefore, the electron transfer resistance $(R c t)$ between the electrolyte and the working electrode surface increase. In this case, the target binding involves an increase of $R c t$ due to the repulsion of the electron from the surface by the negatively charged aptamer. The increase of Rct can be related to the target concentration. On the contrary, Rodriguez et al., ${ }^{107}$ observe a decrease of Rct for the lysozyme aptamer system. The lysozyme protein is positively charged and the interaction with the negative aptamer produces a decrease of the overall negative charge at the electrode surface, which allows an increase of the electron transfer.

Numerous electrochemical aptasensor platforms have been developed for the detection of a wide range of molecules. However, no "gold" standard electrochemical aptasensor protocol exists, specifically for small molecules detection. Which e-chem platform is the most suitable for small molecule detection? Which e-chem system could be the most efficient where no significant specific biorecognition event between the aptamer and the meth occurs (Chapter 3)? For future miniaturization of the system, which e-chem strategy of detection is the most convenient?

Before any considerations related to the cost-effectiveness and practicability of the e-chem strategy to choose. The main point to study is the stability of the system created, as a starting point no signal drift related to buffer incubation is desired. We developed a simple protocol where a buffer solution is used for monitoring in time the electrochemical signal stability of different e-chem platforms. As a consequence, a consistent comparison between different e- 
chem methods is achievable. Some Meth concentrations are injected into the system in parallel to the buffer stability study.

We found that no readable specific binding signal is observed for each electrochemical method strategy tested. Indeed, all generate a significant signal background that is not overcome by the specific binding signal, this was projected to the inconsistency in the self-assembled monolayer (SAM) formation. Despite this finding, the electrochemical impedance spectroscopy (EIS) using a thiolated modified aptamer and the ferri/ferrocyanide redox couple appears as the most appropriate e-chem aptasensor strategy for the development of a reliable portable methamphetamine detection system. The electrochemical impedance spectroscopy strategy offers several advantages over the other e-chem methods. The SAM formed could be designed differently and some optimization could be done and monitored via EIS. As the EIS signal involve the electrode-electrolyte interface, more robust control experiments could be designed (Chapter 5). 


\subsection{Results and discussion}

\section{Electrochemical impedance spectroscopy (EIS) and cyclic voltammetry (CV) confirm gold surface functionalisation}

For each electrochemical strategy used in the next sections, the same SAM is used. It consists of a separated incubation of a thiolated aptamer solution and a blocking agent (6-mercapto-1hexanol, MCH) solution. The steps of the SAM formation are displayed in Figure 4.4.
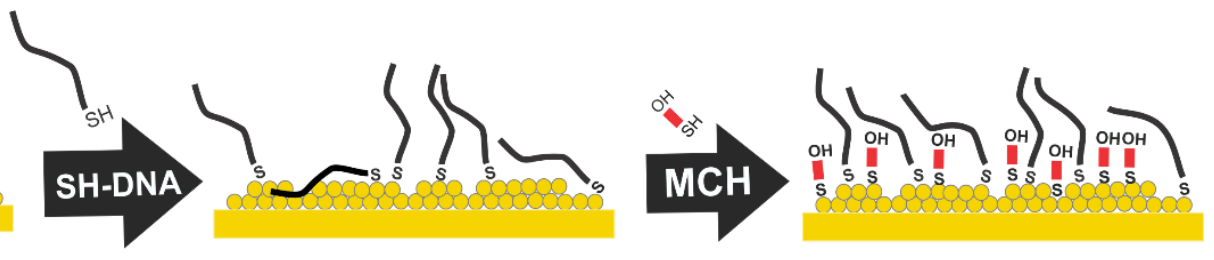

Figure 4.4. Schematic illustration of the formation of a self-assembled monolayer via a separated incubation of thiolated aptamer and $\mathrm{MCH}$ molecule.

The modifications of the gold surface are monitored via EIS and CV measurement with the use of the ferri/ferro redox couple as an electrolyte. As shown in Figure 4.5, EIS and CV measurements are monitored after each modification step.

In Figure 4.5A, the electron transfer resistance $(R c t)$ is the parameter collected via EIS measurement. The Rct of the bare gold electrode is small, unlike the Rct measured with the aptamer fixed and the Rct measured with the aptamer and $\mathrm{MCH}$ fixed. An increase of the Rct is observed following the modification steps, these observations are consistent with previous work done on SAM of aptamer/MCH formation. ${ }^{88,215}$ For a bare gold electrode, the electrodeelectrolyte interface is only related to the mass transfer component (Warburg element). No molecules are fixed on the surface to produce a barrier to the electron transfer between the electrolyte and the working electrode. By adding thiolated aptamer, the electron transfer resistance increases due to the aptamer fixed on the electrode surface. Finally, the Rct increases with the addition of the MCH molecule to the surface.

The same control of the fixation is achievable with cyclic voltammetry (CV) measurements. As observed in Figure 4.5B, the difference between the anodic and cathodic potential $(\triangle E p)$ of the ferri/ferro couple increases for each step of the electrode modification. As shown in Chapter 2, the $\triangle E p$ of a bare gold electrode is $58 \mathrm{mV},{ }^{71,155}$ in Figure 4.5B, the value found 
for the bare gold electrode is $83 \mathrm{mV}$. The difference in the initial value of $\triangle E p$ can be explained by the difference in roughness, the difference in the cleaning process, and the age difference of the electrodes used.

Because the electron transport between the electrode and electrolyte is blocked due to the fixation of molecules on the gold surface, the oxidation and reduction reaction of the ferri/ferro couple will be slowed down. As a consequence, it affords a larger $\triangle E p$ between the oxidation and the reduction peak. Consequently, the maximum $R c t$ and the maximum $\Delta E p$ difference are observed after the final addition of the blocking agent MCH. Each control of SAM formation experiment is achieved before each e-chem strategy tests in the next sections
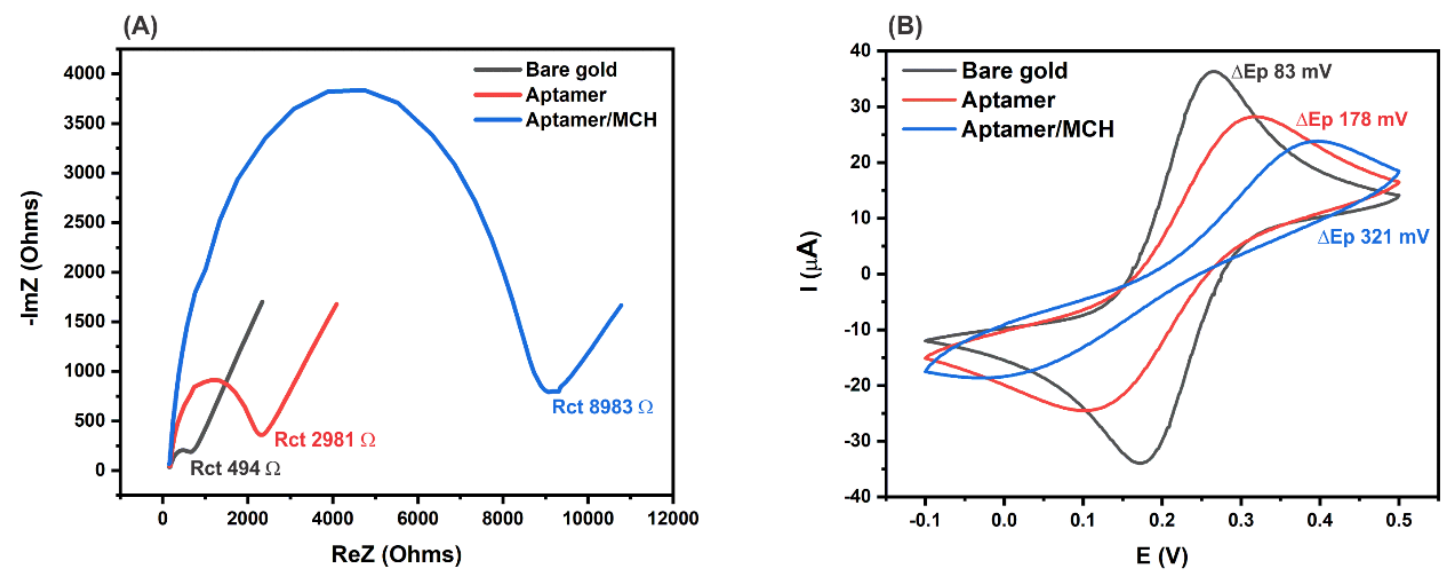

Figure 4.5. Control of the SAM formation via two electrochemical techniques. (A) Electrochemical impedance spectroscopy measurement shows an increase of the electron transfer resistance upon the fixation of aptamer and $\mathrm{MCH}$. (B) An increase of the difference between the anodic and cathodic potential of the ox/red reaction upon SAM formation monitored via cyclic voltammetry.

\section{1 - SWV of methylene blue covalently functionalised aptamer}

For this strategy, the aptamer strand is modified at its 3'-end with a thiol group (- $\mathrm{SH})$, and at its 5 '-end with a methylene blue redox probe. The SAM formation is similar to the protocol explained in the previous section. For the stability and ligand addition measurements, the square wave voltammetry (SWV) measurement technique is used. A range of potential is applied, then a current peak is monitored at the specific redox potential of the MB molecule.

The MB method can provide a "signal on" system or a "signal off" system. If the current related to the methylene blue reduction increases after the target addition, the system is called a signal 
on (Figure 4.6, $\mathrm{e}_{\mathrm{i}}^{-}<\mathrm{e}_{\mathrm{f}}^{-}$). By contrast, if the current related to the MB reduction decreases after the target addition, the system is called a signal off (Figure 4.6, $\left.\mathrm{e}_{\mathrm{i}}^{-}>\mathrm{e}_{\mathrm{f}}^{-}\right){ }^{99}$

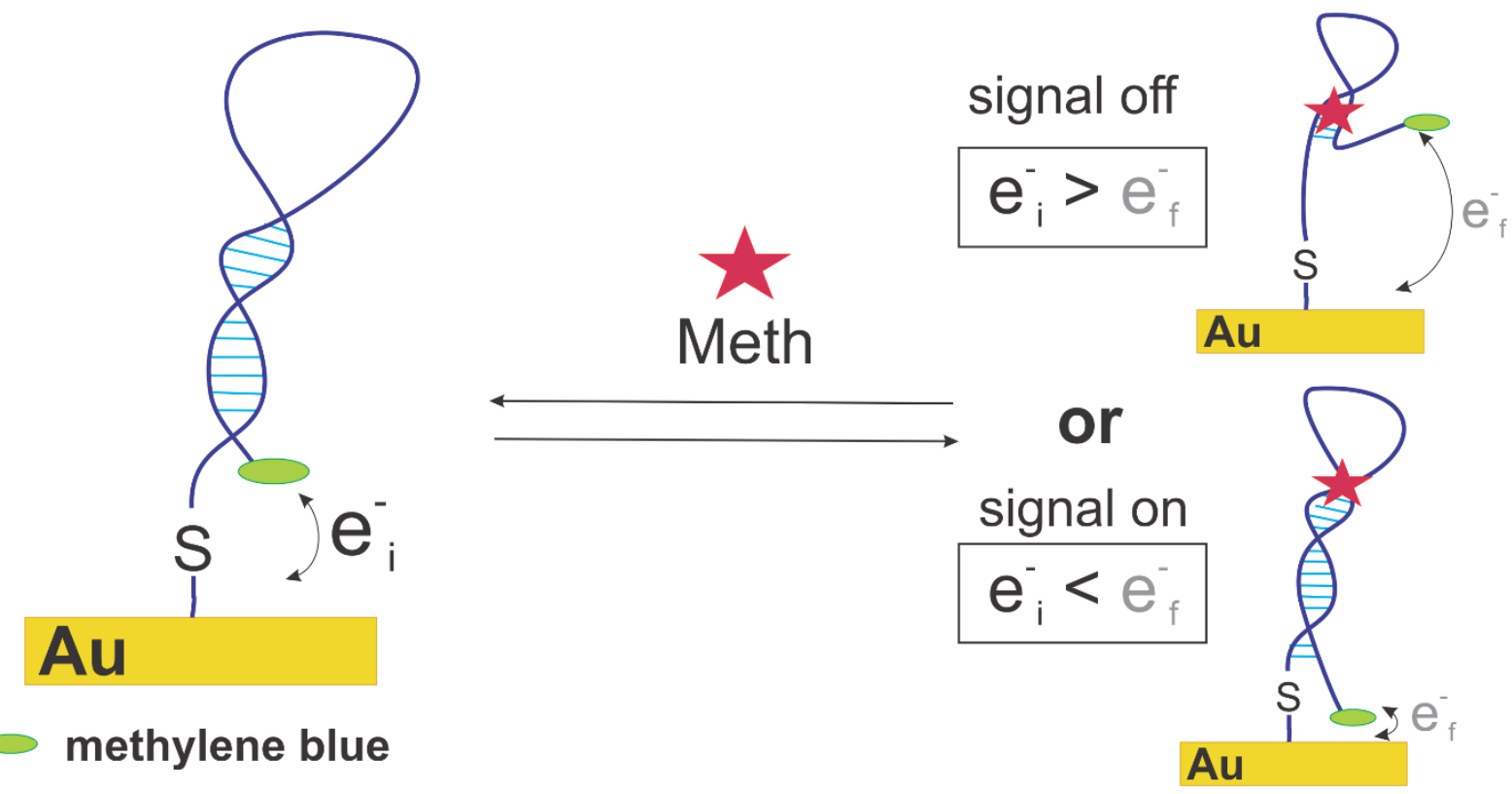

Figure 4.6. Schematic illustration of the methylene blue covalently attached to the thiolated aptamer strategy. The e-chem measurement used is the square wave voltammetry.

One of the specificities of this method is the possibility to modify the square-wave frequency applied for the measurement. As observed in Figure 4.7A, the redox current can be modulated with different square wave-frequency applied. In Chapter 2, we described the square wave voltammetry as a measurement where a "staircase" potential waveform is applied, and a current is monitored at the end of each square pulse. By modulating the frequency of measurement, the sensitivity of the signal can be tuned. ${ }^{216}$

The three frequencies 55, 305, and $505 \mathrm{~Hz}$ have been tested for the buffer and target experiments. A buffer incubation is done in parallel with a target incubation. At $30 \mathrm{~min}$, a first target measurement is done, then a gradual increase of the concentration every $10 \mathrm{~min}$ is achieved until reaching $50 \mu \mathrm{M}$ Meth at $60 \mathrm{~min}$.

As shown in Figure 4.7B and Figure 4.7C, a signal decrease is monitored for $55 \mathrm{~Hz}$ and 305 $\mathrm{Hz}$ during the buffer and target incubation. No difference occurs when the first target incubation is done. By contrast in Figure 4.7D, a gradual increase occurs for $505 \mathrm{~Hz}$ upon buffer and target addition. An increase appears for the $505 \mathrm{~Hz}$ measurement because of the frequency dependency of the signal described previously. Dauphin-Ducharme et al. ${ }^{216}$ show 
that it is possible to design a "signal on" or a "signal off" system by tuning the square wave frequency.
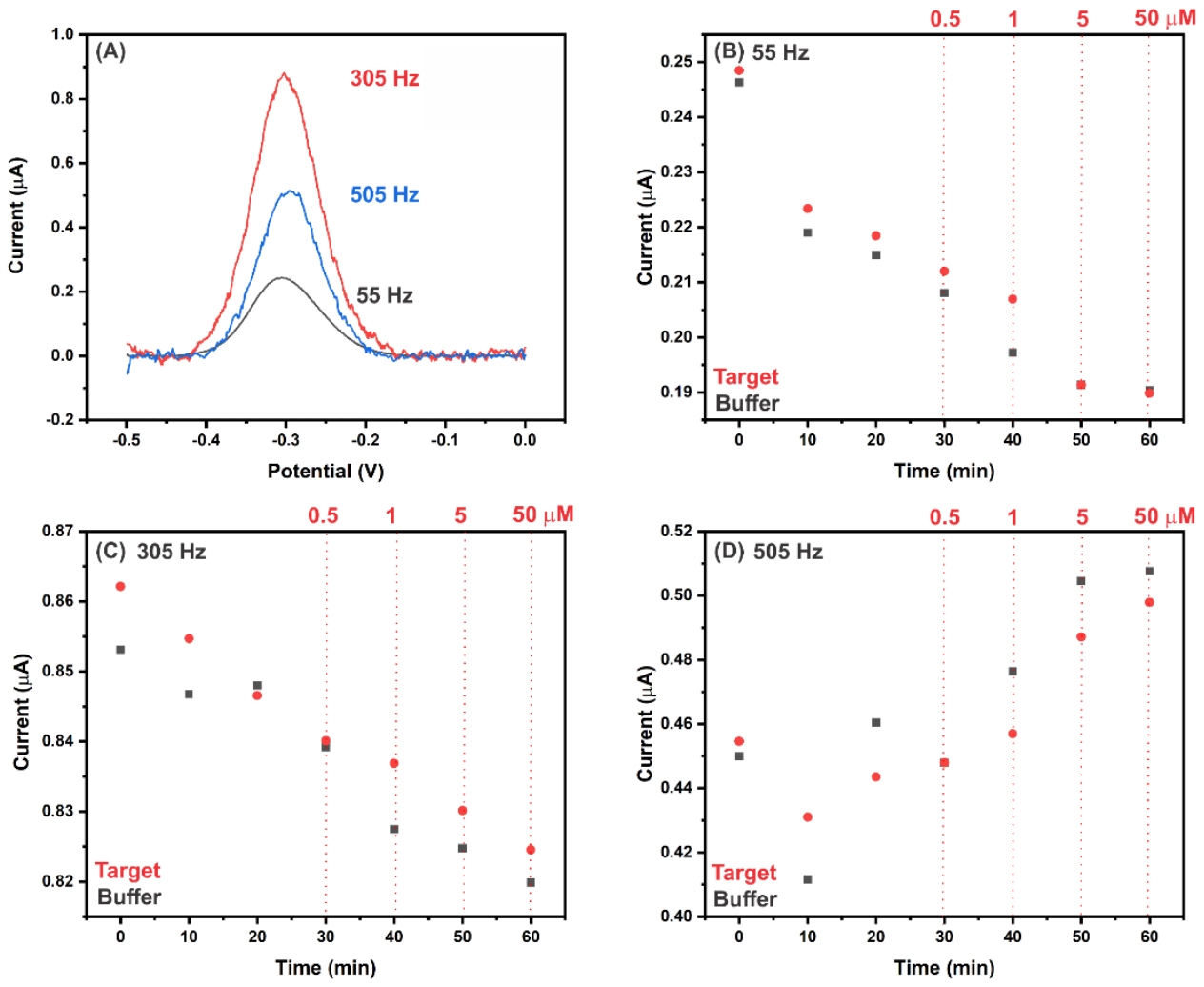

Figure 4.7. (A) The current signal dependency to the square wave frequency used for the SWV measurement. Three different frequency are used (B) $55 \mathrm{~Hz}$, (C) $305 \mathrm{~Hz}$ and (D) $505 \mathrm{~Hz}$ to monitor a buffer and a target signal in function of the time.

A hypothetic explanation of the signal instability is the partial disruption of the SAM due to the large negative potential range applied $(-0.5 \mathrm{~V}$ to $0 \mathrm{~V})$. As a consequence, the aptamer layer which is negatively charged can be affected by the negative potential applied. In addition, with voltammetry techniques, it is not possible to attain a steady-state system resulting in an impact on the signal measured. ${ }^{72,202}$

One solution to overcome the instability would be to create a SAM with a smaller quantity of thiolated aptamer attached on the gold electrode as explained in Josephs et al. ${ }^{121}$ The use of a smaller quantity of aptamer has already been studied by White et al. ${ }^{217}$ They showed that the difference of the intensity of the methylene blue current between the unbound and bound state is enhanced by using a lower aptamer density. However, their case is related to a structural 
change aptamer and hence it cannot be transposed to the minor structural change methamphetamine aptamer studied here.

Furthermore, this technique is efficient for a significant tail-end structure change of the aptamer induced by the target addition. If a structural change occurs in the middle of the stem of the aptamer, this e-chem technique won't be the most suitable for detection. Without any modifications in the SAM formation protocol, the instability related to buffer incubation observed doesn't afford a reliable measurement signal. In the following paragraph, another method will be studied.

\section{2 - DPV of methylene blue non-covalently functionalised aptamer}

The second e-chem strategy tested involves the methylene blue redox probe non-covalently attached to the DNA strand. As explained previously, the methylene blue molecule can interact in different manners with the DNA strand. Intercalation, electrostatic interaction, or minor/major groove binding are possible. ${ }^{205}$ Because of this significant affinity, the methylene blue could be a good probe to monitor the change occurring into the DNA strand during a binding event. Moreover, it is an electroactive species that could be reversibly oxidized and reduced by applying a specific potential.

A similar fixation method of thiolated aptamer and $\mathrm{MCH}$ molecule is used than previously, and the SAM formed is controlled via EIS and CV. After fixation of the aptamer and $\mathrm{MCH}$, the system is incubated in a solution of methylene blue (see Materials and Methods). The whole DNA strand is saturated with MB molecules. ${ }^{204}$ Finally, the target and buffer measurements are done in a buffer solution.

The system obtained is schematized in Figure 4.8. The measurement method used is the differential pulse voltammetry (DPV), and at MB redox potential, a current signal will be monitored. ${ }^{207}$ As observed in Figure 4.8, the current signal upon ligand addition is expected to be smaller than the initial signal current. The ligand-binding event will produce a release of MB molecules due to a structural change of the aptamer or due to a competition of affinity between the Meth and the MB molecule with the DNA strand. Consequently, the current signal will decrease after ligand addition. 

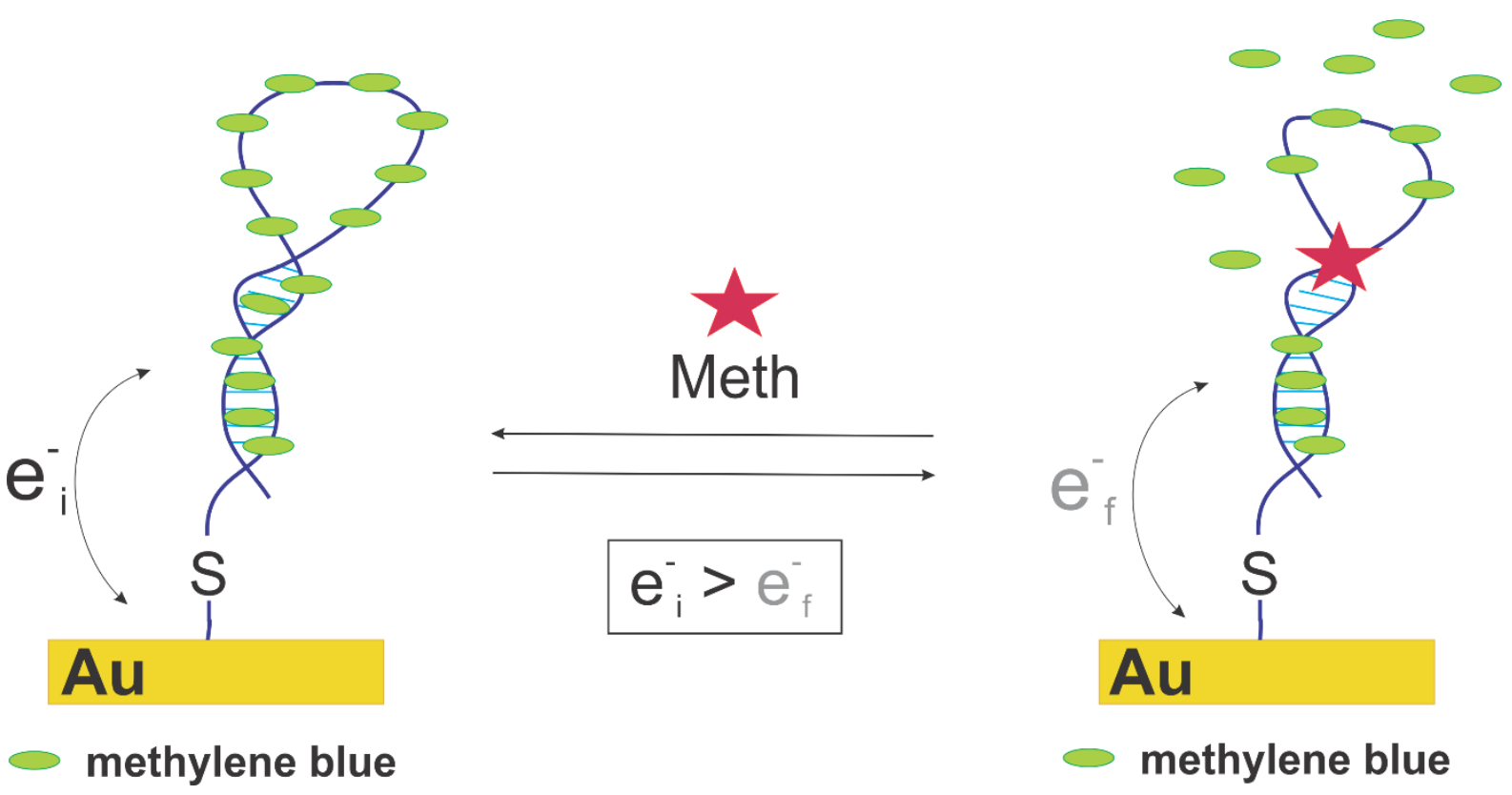

Figure 4.8. Schematic illustration of the methylene blue non-covalently attached to the thiolated aptamer strategy. The e-chem measurement used is the differential pulse voltammetry.

The results obtained are shown in Figure 4.9. In Figure 4.9A, the redox peak of the methylene blue molecule is represented. The first observation in comparison to the MB covalently strategy is that the redox potential of the MB molecule is different. The explanation could be provided by the difference in the measurement method used or/and the difference in the MB molecule purity used. The second observation is that the current signal monitored is smaller. This could be due to the diminished sensitivity of the e-chem used (DPV is less sensitive to SWV). ${ }^{72}$

A similar protocol to the previous experiment is used to monitor the stability of the SAM created. At $30 \mathrm{~min}$, a first target incubation is measured then a gradual increase of Meth concentration is done until $50 \mu \mathrm{M}$ of Meth at $60 \mathrm{~min}$. A control experiment was achieved without an aptamer in solution. As observed in Figure 4.9B, control (blue dots) shows that few MB molecules are deposited on the gold electrode surface. As a consequence, a little contribution of the current signal from these MB molecules is monitored in the overall current signal.By comparing the target and the buffer experiments, a decrease is monitored for both experiments. No clear difference between the two experiments is indicative of a target binding. 
The current signal decrease could be explained as follows: by applying a potential range, there is a disruption of the SAM formed and, thus the MB molecules non-covalently attached to the DNA strand are released. As a consequence, the current signal decreases.
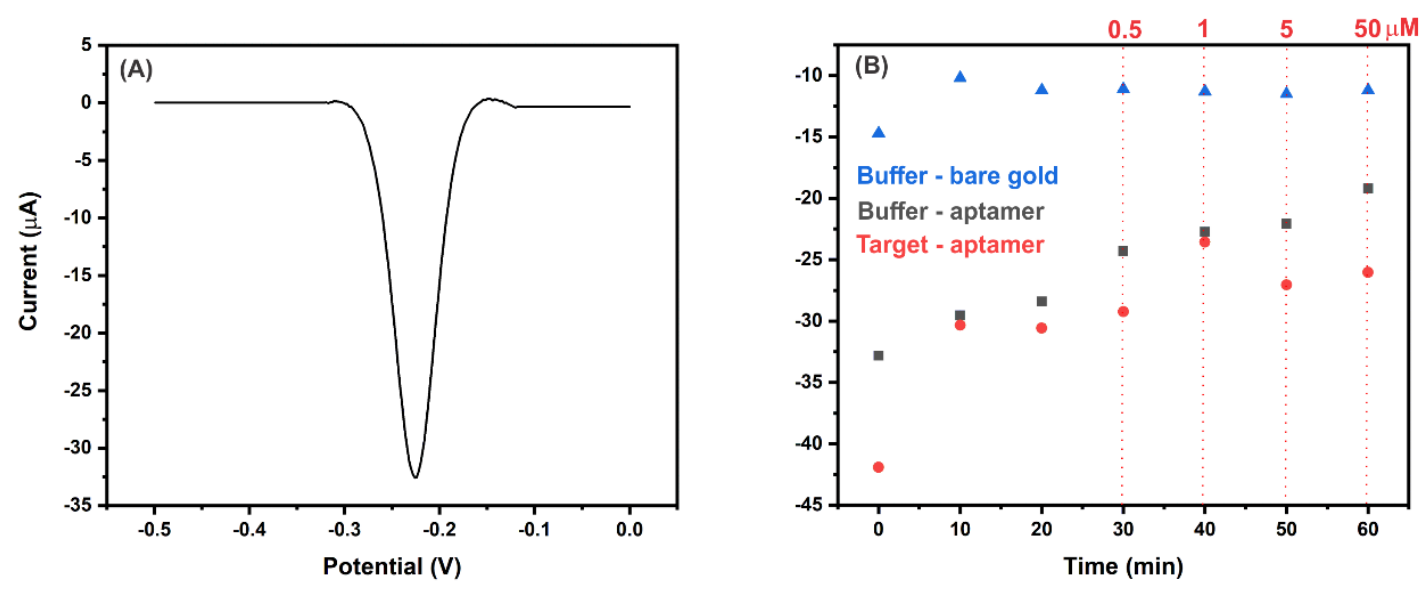

Figure 4.9. (A) Representation of the redox peak of the methylene blue molecule obtained via differential pulse voltammetry. The MB molecule interacts non-covalently with the aptamer strand. (B) MB redox current signal in the function of the time for a buffer, target and buffer without aptamer experiments.

The two voltammetry techniques using the methylene blue redox probe are not the most suitable measurement techniques in our case. The significant fluctuations observed in the two measurements (SWV and DPV) could be produced by the partial disruption of the SAM because of the significant potential range applied. A less disruptive measurement method must be investigated.

\section{3 - EIS of label-free aptamer with redox couple in solution}

The last strategy exploited in this chapter is the electrochemical impedance spectroscopy measurement. By contrast to the two previous strategies tested, the redox probe used is the ferri/ferro redox couple (Figure 4.3). The redox probe is in solution and it doesn't interact with the DNA strand. The signal measured, unlike the MB techniques used previously, is not a current signal. Here, an impedance signal is monitored and is related to the electron transfer that occurs between the electrolyte and the working electrode where the SAM is fixed (Figure 4.10). The system can be separated into two groups: the "signal on" system where $e_{i}{ }^{-}<e_{f}$ and 
the "signal off" system where $e_{i}^{-}>e_{f}$. The structural change of the aptamer upon target addition will induce either an increase in the Rct or a decrease in the Rct.

A direct current potential $\left(E_{D C}\right)$, specific to the redox couple used, is applied. Then, a small sinusoidal alternating current potential $\left(E_{A C}\right)$ is employed to monitor the system at a close steady-state. Consequently, the EIS method is a non-disruptive e-chem method by contrast to voltammetry techniques. As a consequence, it seems worthy to study the EIS system for a future aptasensor application. ${ }^{103,159}$ A similar protocol of fixation is used, and the SAM formed is controlled via EIS and CV measurements.
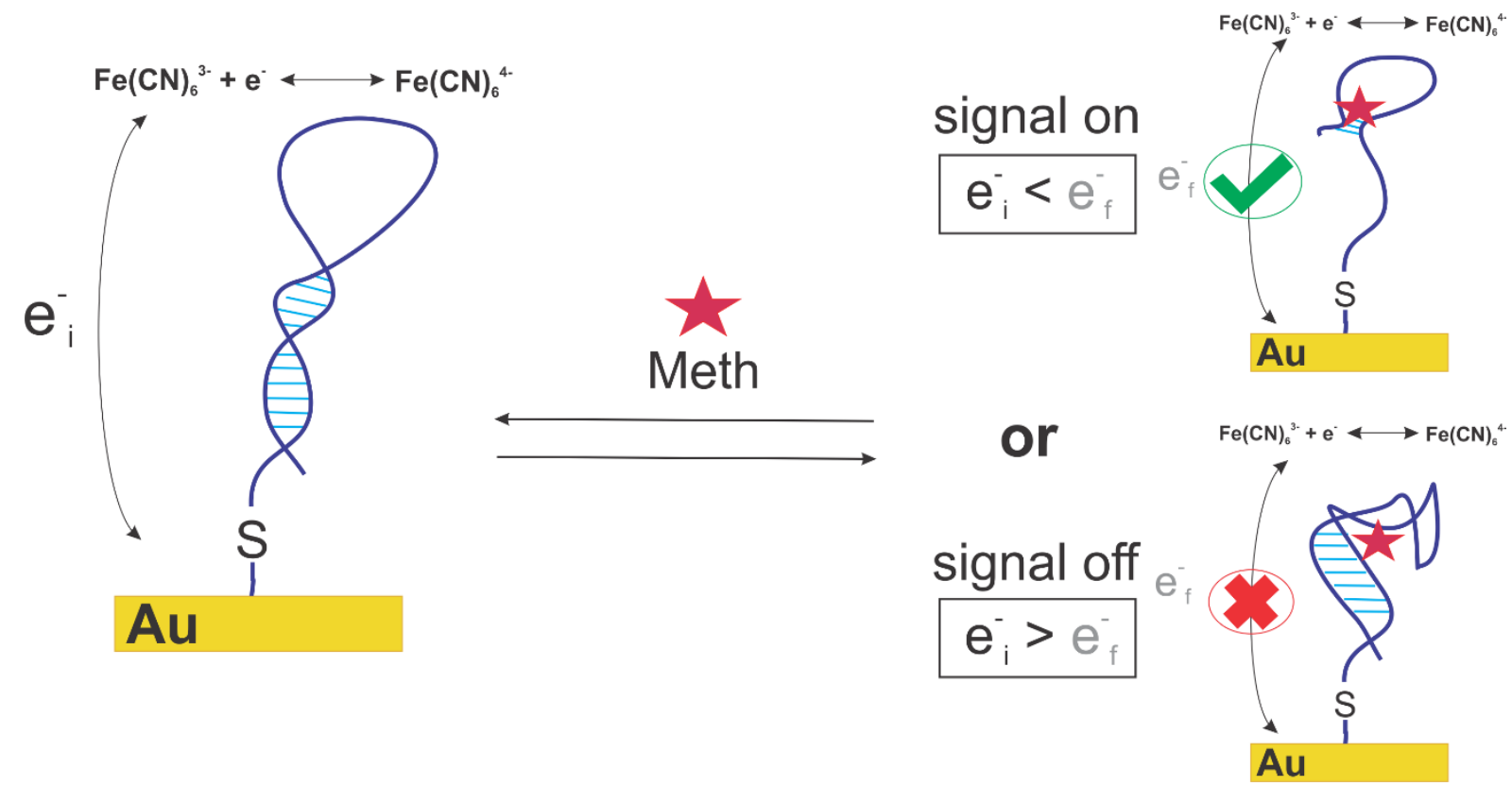

Figure 4.10. Schematic illustration of the thiolated aptamer strand with the ferri/ferro redox couple strategy. The e-chem measurement is the electrochemical impedance spectroscopy.

As observed in Figure 4.11, the buffer and the target measurement signal change similarly over time. No significant difference occurs upon Meth addition. 


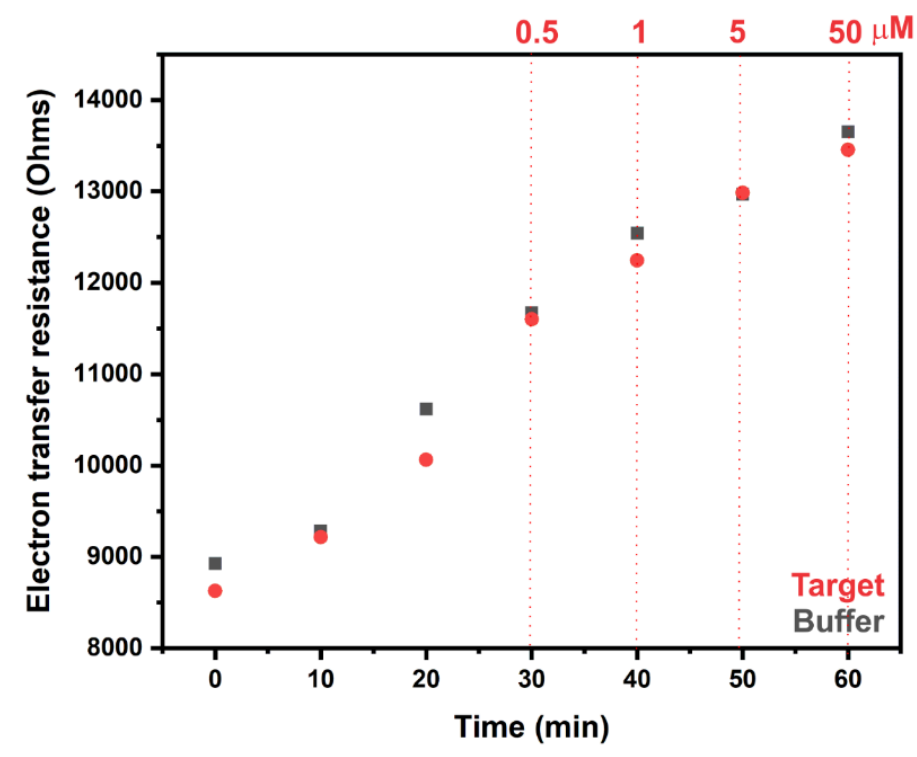

Figure 4.11. Electron transfer resistance in the function of the time for a buffer and a target experiment. The electrochemical measurement technique used is the electrochemical impedance spectroscopy.

The EIS is a less disruptive method than the two other voltammetry techniques studied in this chapter, however, a large signal drift related to the buffer addition appears with EIS too. This is an indication that the problem is not just related to the electrochemical used but could also be related to the fixation and the stability of the SAM created. 


\subsection{Chapter conclusion}

None of the three e-chem strategies considered in this chapter provide a stable e-chem buffer signal. One explanation related to the voltammetry techniques is that the potential range used is too large and, hence provides a significant disruption of the SAM created on the gold electrode surface. The EIS technique, which affords a smaller e-chem perturbation of the system, still shows a signal drift related to the buffer incubation. It can be concluded that the protocol used for the SAM formation on the gold electrode surface with thiolated aptamer and MCH molecule is not suitable for obtaining a stable signal. Moreover, in the context of small molecule detection with an aptamer that doesn't produce a significant bio-recognition event (Chapter 3), no transduced electrochemical signal, related to the binding event, may occur to overcome the instability seen for the different e-chem strategy.

With the two challenges related to the SAM instability and the non-structural change aptamer mentioned, we decided to choose the electrochemical impedance spectroscopy for further investigations of the SAM formation. It is an e-chem technique that doesn't require a modified redox probe aptamer and it can be used specifically to investigate and optimize a SAM formation. The following chapters will provide an insight into the SAM stability optimization and the amplification of the bio-recognition event signal produced by the modest structural change aptamer via EIS. 


\subsection{Experimental section}

\section{Materials}

AuramerBio (Wellington, New Zealand) selected the 75 nucleotides long Meth aptamer via “Affinity Matrix Selex". ${ }^{192} 5$ '-end thiolated aptamer was obtained from AlphaDNA (Montreal, Canada). DNA strand was purified by SDS-PAGE. The binding washing buffer (BWB) was Tris(hydroxymethyl)aminomethane hydrochloride buffer ( $2 \mathrm{mM}$ Tris- $\mathrm{HCl}, 10 \mathrm{mM} \mathrm{NaCl}, 0.5$ $\mathrm{mM} \mathrm{KCl}, 0.2 \mathrm{mM} \mathrm{MgCl}_{2}$, and $0.1 \mathrm{mM} \mathrm{CaCl}_{2} \mathrm{pH}$ 7.5). The assembling buffer (AB) used for the fixation of the solution was prepared with $10 \mathrm{mM} \mathrm{Na} 2 \mathrm{HPO} 4,1 \mathrm{M} \mathrm{NaCl}$ and $1 \mathrm{mM} \mathrm{MgCl} 2$ (pH 7.3). Electrochemical measurements were performed at room temperature $20{ }^{\circ} \mathrm{C}$ using a commercial potentiostat PalmSens3 (PalmSens BV, Netherlands). A standard three-electrode cell containing a gold $(\mathrm{Au})$ working electrode, a Pt counter electrode and an $\mathrm{Ag} / \mathrm{AgCl}$ (3 M $\mathrm{KCl}$ ) reference electrode. $0.1 \mathrm{M}$ phosphate buffer (PBS, $\mathrm{pH}$ 7.0) containing $5 \mathrm{mM}$ $\mathrm{K}_{3}\left[\mathrm{Fe}(\mathrm{CN})_{6}\right] / \mathrm{K}_{4}\left[\mathrm{Fe}(\mathrm{CN})_{6}\right](1: 1, \mathrm{v} / \mathrm{v})$ was chosen as the supporting electrolyte for the EIS measurements. BWB has been used as the supporting measurement buffer for the SWV and DPV measurements. Before any experimentation, the thiolated aptamer was denatured at 95 ${ }^{\circ} \mathrm{C}$ for $5 \mathrm{~min}$ followed by cooling in an ice bath for $10 \mathrm{~min}$. Meth-hydrochloride solutions were prepared freshly in BWB by using a $10 \mathrm{mM}$ stock solution stored at $-20{ }^{\circ} \mathrm{C}$.

\section{Preparation of planar gold electrode}

First, an electrochemical cleaning in a $0.1 \mathrm{M} \mathrm{NaOH}$ solution is done to remove previous thiol bonds. Cyclic voltammetry parameters are the following: 200 cycles, scan rate $1.0 \mathrm{~V} / \mathrm{s}$, from 0.0 to $-1.3 \mathrm{~V}$, E step $0.001 \mathrm{~V}$. Gold electrode is polished for 3 min with a suspension of $0.3 \mu \mathrm{m}$ alumina followed by another polishing step for $3 \mathrm{~min}$ with a suspension of $0.05 \mu \mathrm{m}$ alumina. Then the polished electrode is sonicated for $10 \mathrm{~min}$ in ethanol and for $10 \mathrm{~min}$ in milliQ water to remove any remaining polishing agent. A second electrochemical cleaning in a $0.5 \mathrm{M} \mathrm{H}_{2} \mathrm{SO}_{4}$ solution is used. Cyclic voltammetry parameters are the following: 10 cycles, scan rate $0.1 \mathrm{~V} / \mathrm{s}$, from -0.2 to $1.6 \mathrm{~V}$, E step $0.001 \mathrm{~V}$. Cyclic voltammetry is repeated until 10 scans are stackable between each other. Finally, the cleaning of the gold electrode is checked by using cyclic voltammetry and electrochemical impedance spectroscopy (Chapter 2). 
Aptamer and blocking agent (6-mercapto-1-hexanol, $\mathrm{MCH}$ ) immobilization on gold electrode surface monitored via electrochemical impedance spectroscopy (EIS) and cyclic voltammetry measurement (CV)

$0.1 \mathrm{M}$ phosphate buffer (PBS, pH 7.0) containing $5 \mathrm{mM} \mathrm{K}_{3}\left[\mathrm{Fe}(\mathrm{CN})_{6}\right] / \mathrm{K}_{4}\left[\mathrm{Fe}(\mathrm{CN})_{6}\right](1: 1, \mathrm{v} / \mathrm{v})$ was chosen as the supporting electrolyte for the EIS and CV measurements. After each modification step, an EIS and CV measurement is monitored. EIS parameters are the following: $E_{D C} 0.23 \mathrm{~V}, E_{A C} 0.005 \mathrm{~V}$, tequilibration $4 \mathrm{~s}$, number of frequencies 41 , max frequency $10 \mathrm{kHz}$, min frequency $1.0 \mathrm{~Hz}$. CV parameters are the following: $E_{\text {start }} 0.5 \mathrm{~V}, E_{\text {end }}-0.1 \mathrm{~V}$, number of scans 3 , scan rate $0.1 \mathrm{~V} / \mathrm{s}, E_{\text {step }} 0.001 \mathrm{~V}$, tequilibration $4 \mathrm{~s}$.

\section{Methylene blue covalently attached to the end of the thiolated aptamer strand - SWV measurement used: $1^{\text {st }}$ e-chem strategy studied}

A 5'end thiol and 3'end methylene blue modified aptamer was used. All thiol-MB aptamer solutions are prepared in the assembling buffer. Preparation of $100 \mu \mathrm{L}$ is done for every concentration used. For example, the preparation of $1 \mu \mathrm{M}$ thiol aptamer solution is achieved as follows. $1 \mu \mathrm{L}$ of the aptamer stock $(100 \mu \mathrm{M})$ is mixed with $99 \mu \mathrm{L}$ of assembling buffer then the mixture is heated at $95^{\circ} \mathrm{C}$ for $5 \mathrm{~min}$ and cooled down in an ice bath for $10 \mathrm{~min}$. The solution is spun down for $1 \mathrm{~min}$ at $9000 \mathrm{rpm}$ and stirred for $30 \mathrm{~s}$. The solution needs to re-equilibrate at room temperature for $20 \mathrm{~min}$ at least. A $60 \mu \mathrm{L}$ of thiol-MB aptamer solution prepared is incubated on a clean gold electrode for at least $16 \mathrm{~h}$. After $16 \mathrm{~h}$, the modified electrode is washed $3 \mathrm{x}$ with the assembling buffer solution. Then $60 \mu \mathrm{L}$ of $1 \mathrm{mM} \mathrm{MCH}$ in $\mathrm{AB}$ is incubated for $1 \mathrm{~h}$ on the modified gold electrodes. Finally, the modified electrode is washed $5 \mathrm{x}$ with the $\mathrm{AB}$ solution then it is placed in the BWB working solution. After $10 \mathrm{~min}$ in the working solution used, the first SWV measurement can be done. SWV parameters are the following: $E_{\text {start }}-0.5 \mathrm{~V}, E_{\text {end }} 0.0 \mathrm{~V}, E_{\text {step }} 0.001 \mathrm{~V}, E_{\text {amplitude }} 0.05 \mathrm{~V}$, and frequencies used 55,305 and 505 $\mathrm{Hz}$.

\section{Methylene blue non-covalently attached to the thiolated aptamer strand - DPV measurement used: $2^{\text {nd }}$ e-chem strategy studied}

All thiol aptamer solutions are prepared in the assembling buffer. Preparation of $100 \mu \mathrm{L}$ is done for every concentration used. For example, the preparation of $1 \mu \mathrm{M}$ thiol aptamer solution is 
achieved as follows. $1 \mu \mathrm{L}$ of the aptamer stock $(100 \mu \mathrm{M})$ is mixed with $99 \mu \mathrm{L}$ of assembling buffer then the mixture is heated at $95{ }^{\circ} \mathrm{C}$ for $5 \mathrm{~min}$ and cooled down in an ice bath for $10 \mathrm{~min}$. The solution is spun down for $1 \mathrm{~min}$ at $9000 \mathrm{rpm}$ and stirred for $30 \mathrm{~s}$. The solution needs to reequilibrate at room temperature for $20 \mathrm{~min}$ at least. A $60 \mu \mathrm{L}$ of thiol aptamer solution prepared is incubated on a clean gold electrode for at least $16 \mathrm{~h}$. After $16 \mathrm{~h}$, the modified electrode is washed 3x with the assembling buffer solution. Then $60 \mu \mathrm{L}$ of $1 \mathrm{mM} \mathrm{MCH}$ in $\mathrm{AB}$ is incubated for $1 \mathrm{~h}$ on the modified gold electrodes. Finally, the modified electrode is washed 5x with the $\mathrm{AB}$ solution then it is placed in the BWB working solution. The ssDNA-modified electrodes were immersed for $20 \mathrm{~min}$ in a $3 \mathrm{mM}$ solution of $\mathrm{MB}$, followed by washing with BWB. Then placed in BWB for further e-chem characterization. DPV parameters are the following: $E_{\text {start }}$ $0.5 \mathrm{~V}, E_{\text {end }} 0.0 \mathrm{~V}, E_{\text {amplitude }} 0.04 \mathrm{~V}, E_{\text {step }} 0.01 \mathrm{~V}$, and scan rate $0.02 \mathrm{~V} / \mathrm{s}$.

\section{Thiolated aptamer strand with the use of the ferri/ferro couple - EIS measurement used: $3^{\text {rd }}$ e-chem strategy studied}

All thiol aptamer solutions are prepared in the assembling buffer. Preparation of $100 \mu \mathrm{L}$ is done for every concentration used. For example, the preparation of $1 \mu \mathrm{M}$ thiol aptamer solution is achieved as follows. $1 \mu \mathrm{L}$ of the aptamer stock $(100 \mu \mathrm{M})$ is mixed with $99 \mu \mathrm{L}$ of assembling buffer then the mixture is heated at $95{ }^{\circ} \mathrm{C}$ for $5 \mathrm{~min}$ and cooled down in an ice bath for $10 \mathrm{~min}$. The solution is spun down for $1 \mathrm{~min}$ at $9000 \mathrm{rpm}$ and stirred for $30 \mathrm{~s}$. The solution needs to reequilibrate at room temperature for $20 \mathrm{~min}$ at least. A $60 \mu \mathrm{L}$ of thiol aptamer solution prepared is incubated on a clean gold electrode for at least $16 \mathrm{~h}$. After $16 \mathrm{~h}$, the modified electrode is washed 3x with the assembling buffer solution. Then $60 \mu \mathrm{L}$ of $1 \mathrm{mM} \mathrm{MCH}$ in $\mathrm{AB}$ is incubated for $1 \mathrm{~h}$ on the modified gold electrodes. Finally, the modified electrode is washed $5 \mathrm{x}$ with the $\mathrm{AB}$ solution then it is placed in the BWB working solution. After $10 \mathrm{~min}$ in the working solution used, the first EIS measurement can be done. EIS parameters are the following: $E_{D C}$ $0.23 \mathrm{~V}, E_{A C} 0.005 \mathrm{~V}, t_{\text {equilibration }} 4 \mathrm{~s}$, number of frequencies 41 , maximum frequency $10 \mathrm{kHz}$, and minimum frequency $1.0 \mathrm{~Hz}$. 


\section{CHAPTER 5}

\section{Self-assembled monolayer investigation via electrochemical impedance spectroscopy: stability and aptamer density optimization}

\subsection{Abstract}

Electrochemical (e-chem) aptasensors have emerged as a promising class of biosensors capable of detecting a wide range of molecular analytes due to their sensitivity, specificity, and practicability. Self-assembled monolayer (SAM) formation on the electrode is the first critical step for the development of e-chem aptasensors. Sulfur-gold self-assembled monolayers preparation involving covalent binding between a thiolated aptamer with a gold surface is most commonly used. However, the disordered aptamer conformation and the limited aptamer density value reached on the electrode are important problems for aptasensors prepared in this way. As a result, signal drift related to the buffer incubation occurs and no detection signal can be observed.

Here, we combine electrochemical impedance spectroscopy (EIS) and chronocoulometry (CC) methods to investigate different protocols of aptamer fixation on a gold electrode to enable the formation of a stable and high aptamer density SAM. We found that the co-incubation of the thiolated aptamer and the blocking agent improves the SAM stability by creating a more ordered monolayer. Despite the stability enhancement afforded by the co-incubation protocol, 
the aptamer density significantly decreases and may disable a specific detection signal. However, due to inter-aptamer steric hindrance, the aptamer density is limited and consequently cannot be maximized with the use of the thiolated protocol. A novel protocol, using disulfide aptamers that combines SAM stability and sufficient aptamer density resulting in a target-induced signal, has been developed. Disulfide fixation is a promising strategy to bypass the aptamer density limitation associated with thiolation protocols by fixing two aptamer strands simultaneously to a gold surface. From these findings, we showed that for an e-chem aptasensor, this way of preparing a sensor device exhibits great potential for improving the SAM stability and enhancing the sensitivity of biosensing systems. 


\subsection{Introduction}

Electrochemical (e-chem) aptasensors are one of the most promising transducer systems for small-molecule detection. ${ }^{68,69}$ The crucial component in an e-chem aptasensor is the biorecognition layer on the working electrode surface, typically a self-assembled monolayer $(\mathrm{SAM}) .{ }^{218}$ For this purpose, sulfur-gold chemistry has been widely used for several decades. ${ }^{82}$, $85,219,220$

SAMs of organosulfur compounds (thiols, disulfides, sulfides) form on metal substrates by spontaneous adsorption from a gas or liquid phase. They are dynamic materials that re-organize themselves to complete a well-ordered layer. ${ }^{218}$ The most extensively studied metal substrates are gold, silver, copper, palladium, platinum, and mercury. ${ }^{82}$ Gold is a convenient choice as a solid substrate for SAMs formation; it is easy to obtain, it is unreactive with atmospheric $\mathrm{O}_{2}$, it is biocompatible and finally, it is a popular substrate used for spectroscopies and analytics techniques. ${ }^{82}$

Organosulfur compounds fixed on the gold surface consist of three parts: (a) the sulfur headgroup, which forms a strong bond with the gold surface; (b) the hydrocarbon chain (of variable length) which stabilizes the SAM through van der Waals interactions, and (c) the terminal group which can have different functionalities. ${ }^{86}$ These three structural components contribute to the structure and the chemical and physical properties of the SAMs.

The steps of SAM formation between a gold surface and an organosulfur compound are depicted in Figure 5.1. An initial physisorption event (A) occurs between the hydrocarbon chains and the gold surface through Van der Waals interactions, followed by chemisorption (B) which involves the formation of a strong bond between the sulfur headgroup with the gold surface. ${ }^{89,} 90$ This step is referred to as the "lying-down" phase formation, where the hydrocarbon chains are placed in parallel to the gold surface. A nucleation phase (C) then results in the formation of a "standing-up" phase. Finally, a highly ordered "standing-up" phase (D) is created. ${ }^{86}$

Most investigations of SAM formation are related to alkyl thiol/disulfide compounds (SH$\left.\mathrm{C}_{\mathrm{x}} \mathrm{H}_{\mathrm{y}}-\mathrm{OH} / \mathrm{HO}-\mathrm{C}_{\mathrm{x}} \mathrm{H}_{\mathrm{y}}-\mathrm{S}-\mathrm{S}-\mathrm{C}_{\mathrm{x}} \mathrm{H}_{\mathrm{y}}-\mathrm{OH}\right) .{ }^{82},{ }^{86}$ In contrast, the use of long aptamer strands can involve some difference in the SAM formation. Because the aptamers strands are long and flexible, physisorption on the gold surface is strong in comparison to that of short linear alkyl 
thiol compounds. ${ }^{12}$ Moreover, DNA strands are nitrogen-rich molecules that have a meaningful affinity with the gold surface. ${ }^{59,87}$ Some small alkyl thiol molecules are specifically used for preventing nonspecific adsorption of DNA strands to the gold surface and inducing the formation of a highly ordered "standing-up" layer. Short-chain thiols have a less complex adsorption process on the gold surface because the interactions with the surface are mainly due to the sulfur moiety, and only small contributions from tail-surface and tail-tail interactions occur. $^{221}$ The most common small alkyl thiol molecules used (also known as backfillers) are cysteamine, ${ }^{222}$ 6-mercapto-1-hexanol $(\mathrm{MCH}),{ }^{223} \quad$ 4-mercaptobutanol, ${ }^{224}$ and 3mercaptopropionic acid (MPA). ${ }^{225}$ Di-thiols are also popular for SAM assemblies such as 1,6 - hexanedithiol (HDT), ${ }^{226}$, and 1,8 - octanedithiol (ODT). ${ }^{227}$

(A)

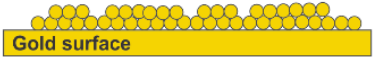

(B)

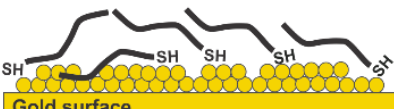

(C)

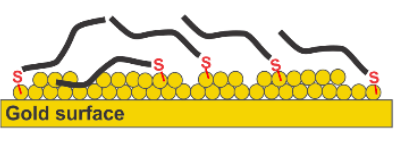

(D)

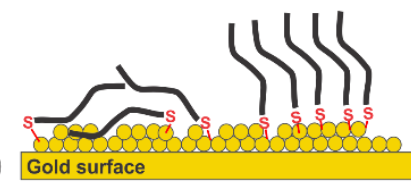

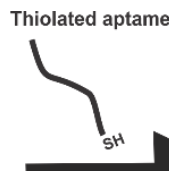
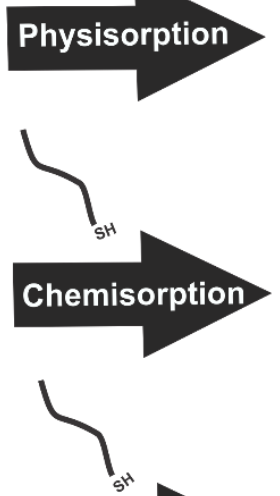

Nucleation

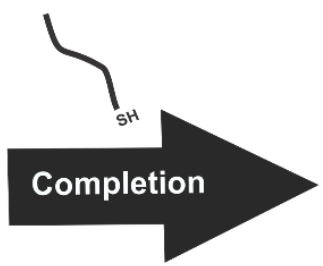

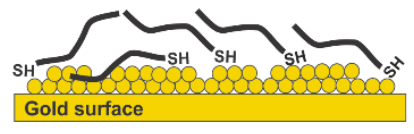
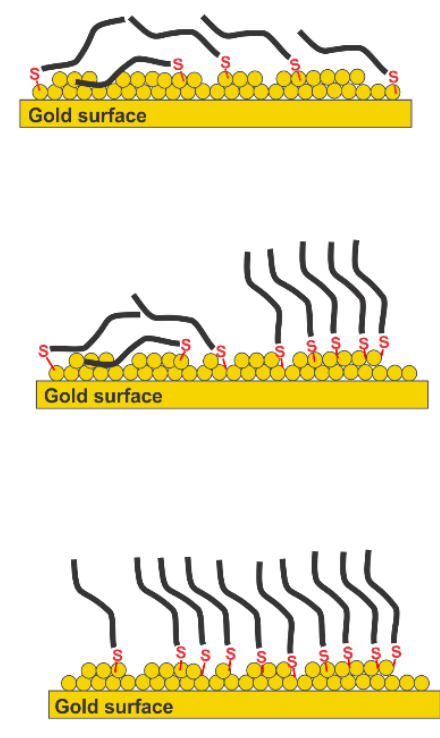

Figure 5.1. Scheme of the different steps occurring during the self-assembly of thiolated DNA strand on gold electrode surface: (A) physisorption, (B) chemisorption "lying-down" phase formation, (C) nucleation of the "standing-up" conformation, and (D) completion of the "standing-up" phase.

Some limitations with the use of thiolated aptamers occur for the SAM stability and the aptamer density fixed on the gold surface. First, the two-step method, including a separated incubation of a thiolated aptamer and a separated incubation of the $\mathrm{MCH}$ backfiller, is popular for e-chem 
aptasensor platform preparation. ${ }^{84,88,160,208}$ The SAM formed, however, is not the most suitable layer for biosensing. Numerous drawbacks appear related to the two-step protocol such as the disorganization of the SAM formed resulting in a signal drift related to the buffer incubation. ${ }^{120 \text {, }}$ 121, 215, 228, 229 Long thiolated aptamers afford two adsorption interactions with the gold surface: chemisorption between the sulfur and the gold, and physisorption between the nitrogen-rich DNA strand and the gold surface. ${ }^{84,121,221}$ The latter interaction creates a "lying-down" structure (striped phase). ${ }^{86,230}$ With the use of the backfiller, aptamers in a "lying-down" position can switch to a "standing-up" position. ${ }^{85,86,221,230,231}$ Unfortunately, the SAM system including thiolated aptamers is more complex and obtaining an ideal "standing-up" layer is not achievable. As a result, control over the aptamer surface conformation with a two-step thiolated aptamer protocol is challenging to achieve.

Secondly, another concerns the optimal aptamer density on the gold surface for aptasensing experiments. Specifically, a large aptamer density on the gold electrode can have an unfavorable effect on the biorecognition event and consequently on the transduced signal. ${ }^{232}$ For a high aptamer density context, the steric hindrance between the aptamer strands prevents structural changes induced by the target (also known as the ligand). It could also prevent the ligand to access the aptamer binding pocket. ${ }^{233}$ However, this case is particular to the aptamers involved in an important structural change and specific to large ligand molecules such as DNA strand. In this context, a minimal aptamer density on the gold surface would be required.

(Figure 5.2, Case 1).
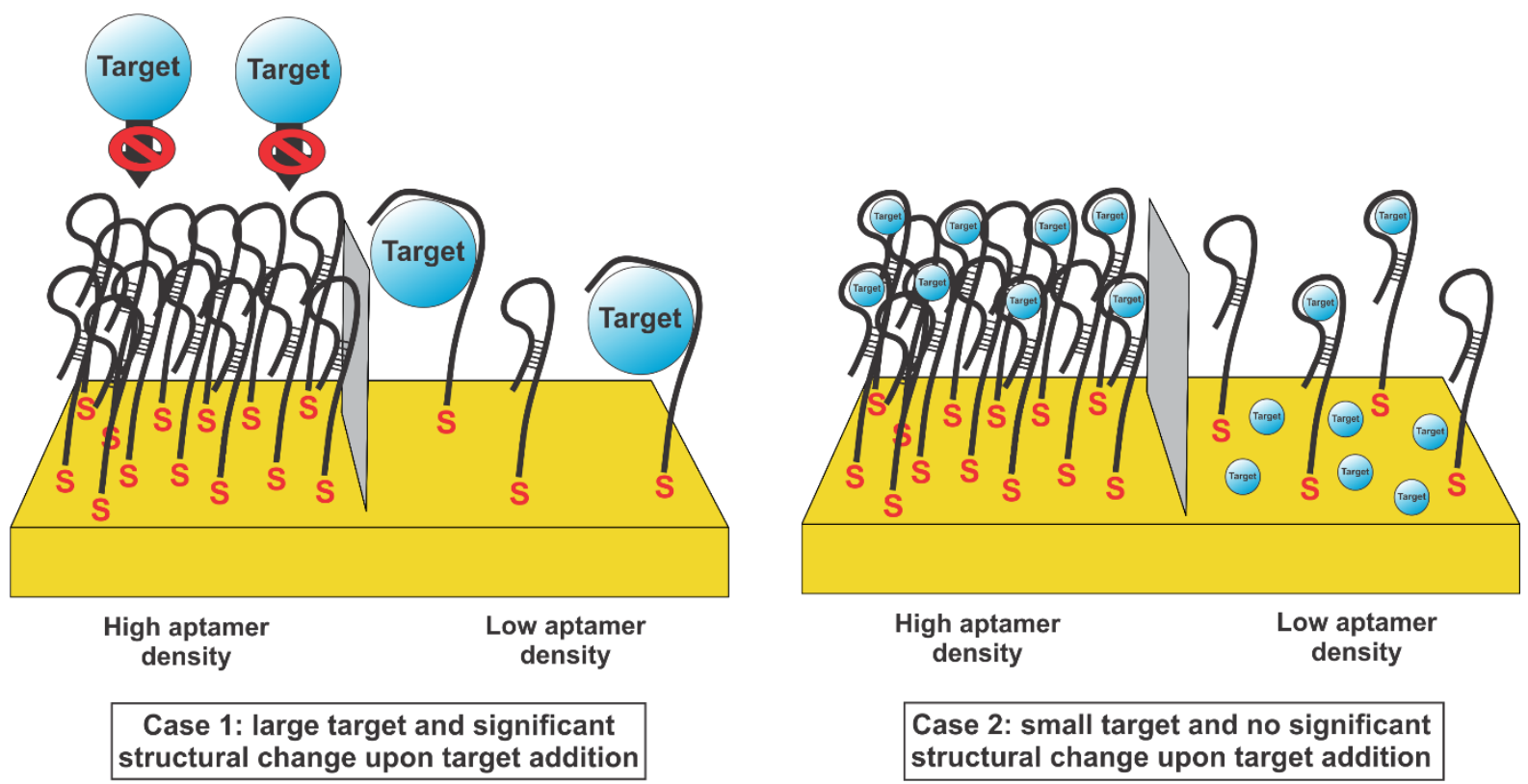

Figure 5.2. Schematic illustration of two e-chem cases of detection strategy on a gold electrode surface. 
Here, the ligand is the methamphetamine (Meth) molecule: a small organic molecule (MW 150 $\mathrm{g} / \mathrm{mol}$ ) with only one functional group. As observed in Chapter 3, the principal interaction between the aptamer and the methamphetamine is entropically driven and doesn't involve a significant structural change of the aptamer (conformational selection binding mechanism). In this context, opposite to the one of Ricci et al. ${ }^{232}$, a high aptamer density is conceived as an amplification system of the detection signal (Figure 5.2, Case 2). The initial electron transfer resistance will be substantial and, therefore, even a weak binding event will be detectable. That's why, in addition to the SAM stability investigation, the maximization of the aptamer density by increasing the aptamer concentration will be investigated in this chapter. ${ }^{234}$ However, the thiol protocol doesn't allow to reach a high aptamer density due to the inefficiency and inconsistency of fixation.

A solution for bypassing the instability produced by the disorder of the SAM formed with the two-step protocol has been explored. Keighley et al. ${ }^{225}$ produced a highly ordered SAM with a co-incubation protocol consisting of the co-incubation of the thiolated aptamer with the $\mathrm{MCH}$ backfiller.

Furthermore, a new two-step protocol has been discovered, where disulfide (S-S) aptamers have been used instead of thiolated aptamers, was developed for the reliable fixation of aptamer strands on the gold surface. Electrochemical impedance spectroscopy (EIS) and the chronocoulometry (CC) methods emerge as the most suitable e-chem methods for the SAM investigation related to the aptamer density and SAM organization. ${ }^{104,159}$

We found that the co-incubation significantly increases the stability of the formed SAM; no signal drift related to the buffer incubation is observable. However, the aptamer density decreases even further in comparison with the two-step thiolated protocol. Consequently, no binding signal occurs upon the methamphetamine addition with the co-incubation protocol. We discovered that increasing the thiolated aptamer concentration incubated in the two-step protocol enhances the SAM stability. However, a limiting aptamer density occurs and shows that the layer formed is still not appropriate for Meth detection. We designed a new fixation protocol consisting of a two-step protocol using disulfide aptamers. A high aptamer density SAM has been demonstrated and good stability of the layer has been proved. Our main hypothesis, to explain why the aptamer density increases, is the following: two aptamers assemble simultaneously on the gold surface in contrast to the single aptamer fixation with the 
thiolated protocol. In addition, a signal related to the Meth addition is monitored via the twostep protocol using disulfide aptamers.

The electrochemical aptasensors system used for small molecule detection involves a lack of sensitivity due to the plausible modest-structural change and entropically driven binding event that occur between the aptamer and the small molecule ligand. Additional fluorescent molecules or conductive materials to enhance the binding signal readability are used. These additional components show efficiency, nevertheless, the overall system becomes more complex and expensive to build and to use. The two protocols investigated in this section show a great improvement in SAM stability for the simple and low-cost system. A considerable enhancement of the aptamer density is demonstrated with the disulfide aptamer protocol (S-S protocol) and could be expanded to other e-chem methods. 


\subsection{Results and discussion}

\subsubsection{Thiolated aptamer strategy}

In the following section, all SAMs have been formed with thiolated modified aptamers. Different protocols will be explored using thiolated aptamers for the SAM instability and SAM aptamer density examination.

\section{The separated fixation of $1 \mu \mathrm{M}$ thiolated aptamer and $1 \mathrm{mM} \mathrm{MCH}$ produces an important signal drift related to the buffer incubation}

The initial SAM formation is produced via the two-step protocol (Figure 5.3) consisting of a separated incubation of $1 \mu \mathrm{M}$ thiolated aptamer and $1 \mathrm{mM}$ of $\mathrm{MCH}$ blocking agent. ${ }^{84,88,208}$
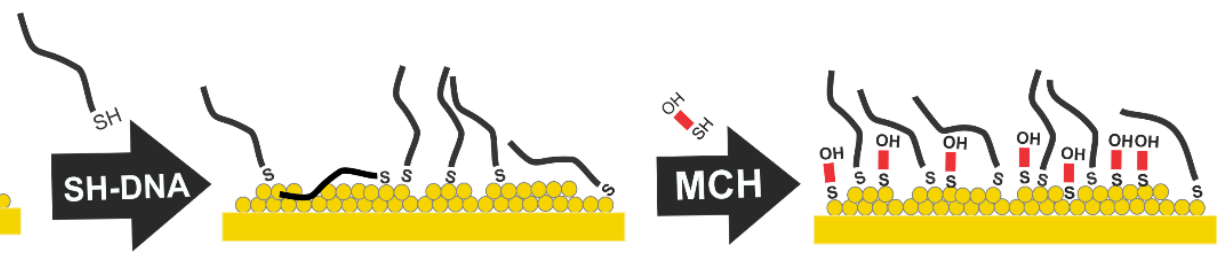

Figure 5.3. Schematic of the two-step thiolated aptamer and $\mathrm{MCH}$ molecule fixation on gold electrode surface.

The aptamer used for the investigation is the Aptamer-2 (aptamer investigated in Chapter 3), and the original length of the Aptamer-2 is 75 nucleotides (75mer). First, the SAM formed is tested for stability in the working buffer (Binding washing buffer, BWB). The measurements done in the BWB (Control) are superimposed with the measurements done in BWB containing a gradual increase of methamphetamine (Meth) concentration (Target). As shown in Figure 5.4, the electron transfer resistance $(R c t)$ values for the buffer and the target experiments increase gradually following the time. No differentiation is visible between the control and the target experiments. 


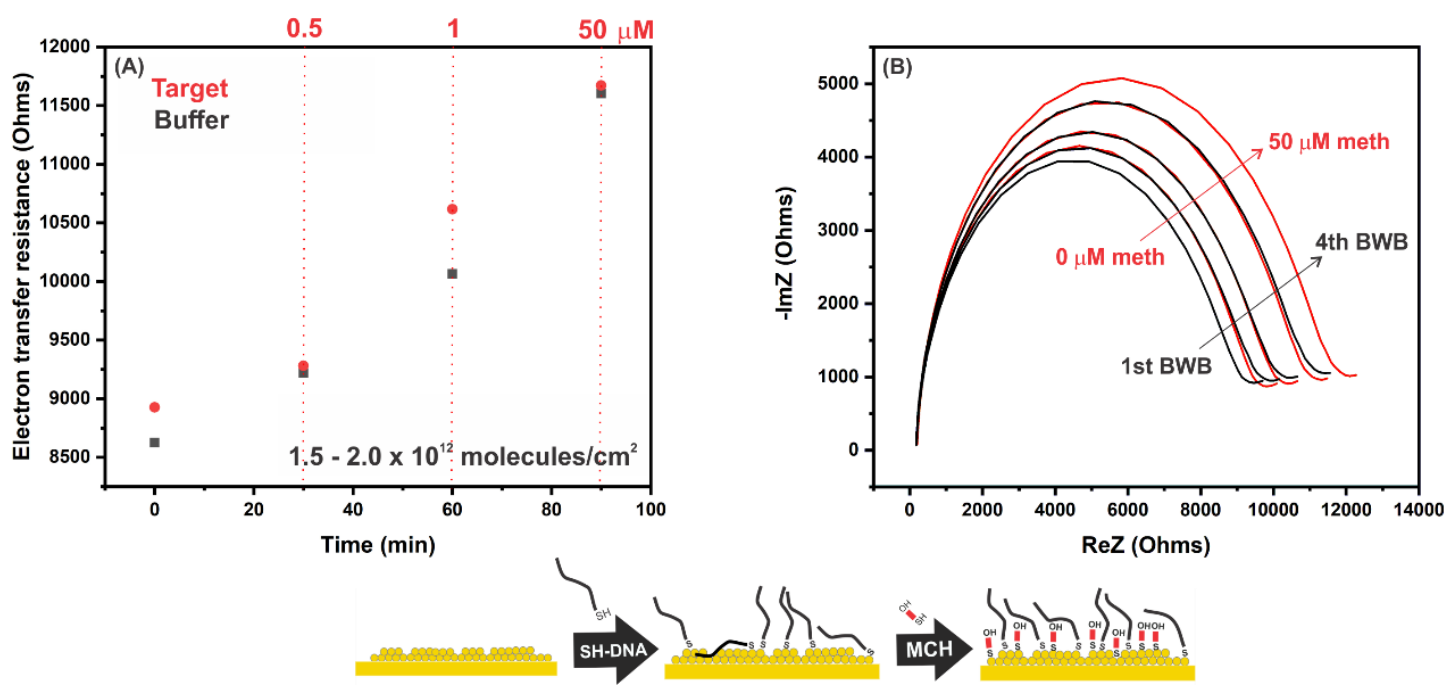

Figure 5.4. EIS results for the two-step protocol with the 75mer aptamer $-1 \mu \mathrm{M}$ incubated. (A) Rct is plotted in function of the time. (B) Raw data of the two-step protocol presented in a Nyquist plot.

The signal drift related to the buffer incubation, in the specific system used, has already been observed and studied. ${ }^{120,121,215,228,229}$ Vogt et al. ${ }^{229}$ and Lazar et al. ${ }^{228}$ assigned the instability observed to the etching of the gold electrode surface because of the adsorption of the cyanide molecules coming from the redox couple potassium ferri/ferrocyanide (ferri/ferro) used as an electrolyte for the EIS experiments. In their studies, a long incubation time of the gold electrode in the ferri/ferro electrolyte has been used. By contrast here, the duration of incubation in the ferri/ferro electrolyte is approximately $2 \mathrm{~min}$ (one EIS measurement), thus the effect of cyanide adsorption resulting in the etching of the gold electrode surface is minimized and consequently negligible. Xu et al. ${ }^{120}$ showed the influence of the temperature on the organization of the SAM and proved that the use of high-temperature induces a re-organization of the SAM formed, resulting in an EIS signal drift measured. Here, the temperature is kept constant at $20{ }^{\circ} \mathrm{C}(+-5$ ${ }^{\circ} \mathrm{C}$ ), thus, the SAM re-organization due to the temperature is avoided.

However, the re-organization of the SAM at room and constant temperature is a mechanism that occurs and has already been studied by Liu et al. ${ }^{235}$ They attributed the instability observed to a lack of ordering into the SAM formed that creates a continuous re-organization of the SAM and generates a signal drift observable with EIS measurement. Furthermore, due to the positive potential applied for the EIS measurement using the ferri/ferro couple, the negative aptamer strands fixed on the gold electrode are electrostatically attracted to the electrode surface resulting in a disordering of the SAM formed which may consist of a mix of "lying-down" and 
"standing-up" conformations. Different approaches in this chapter will be examined for creating a more ordered SAM and, thus, avoiding the signal drift related to the buffer incubation.

\section{The self-assembled monolayer of $\mathrm{MCH}$ shows the most stable behavior}

Several backfiller agents can be used for the formation of a SAM. The two groups of molecules considered here are the alkyl thiol (MCH and MPA) and the alkyl dithiol (HDT and ODT) molecules; they are short and rigid molecules. As a consequence, they are expected to replace the physisorbed DNA strands on the gold surface and thus, affording the formation of a highly ordered aptamer "standing-up" SAM as observed in Figure 5.1D. By exploring the stability in a buffer solution of a pure SAM for each of these agents, we will be ensured to use the most appropriate blocking agent for the following experiments of this chapter in terms of SAM stability.

A pure layer of $\mathrm{MCH}, \mathrm{MPA}, \mathrm{HDT}$, and ODT has been formed and tested in BWB in the function of the time via EIS measurement. The electron transfer resistance has been plotted as a relative electron transfer resistance for being able to compare each blocking agent layer at the same scale, however, caution must be taken when comparing the relative results due to the different initial $R c t$ for each molecule. ${ }^{236}$ In Figure 5.5, the fixation of each blocking agent on a gold electrode surface is represented. As observed in Figure 5.5, the dithiol molecules (HDT and ODT) are fixed horizontally on the gold surface because of their two end thiol groups resulting in a "lying-down" configuration. As a consequence, they cover more the electrode surface by contrast to the "standing-up" configuration of the thiol molecules, and thus dithiol molecules afford a larger initial Rct. The MPA molecule is the blocking agent that shows the higher instability among the four blocking agents tested (the linear fit of the relative $R c t$ values for MPA shows a slope of 0.464). This could be due to the carboxylic acid end group that can interact with the hydrophilic surface of the gold electrode. ${ }^{237}$ 
MCH molecule shows the smaller initial Rct in Figure A.4 and consequently, could provide a large relative $R c t$ change induced by a small $R c t$ change. ${ }^{236}$ However, the most stable trend of relative $R c t$ in the function of the time is given by $\mathrm{MCH}$. Indeed, the linear fit of the relative $R c t$ values for MCH shows a slope of -0.0720 . As a consequence, $\mathrm{MCH}$ blocking agent will be used for the following investigations on the SAM.
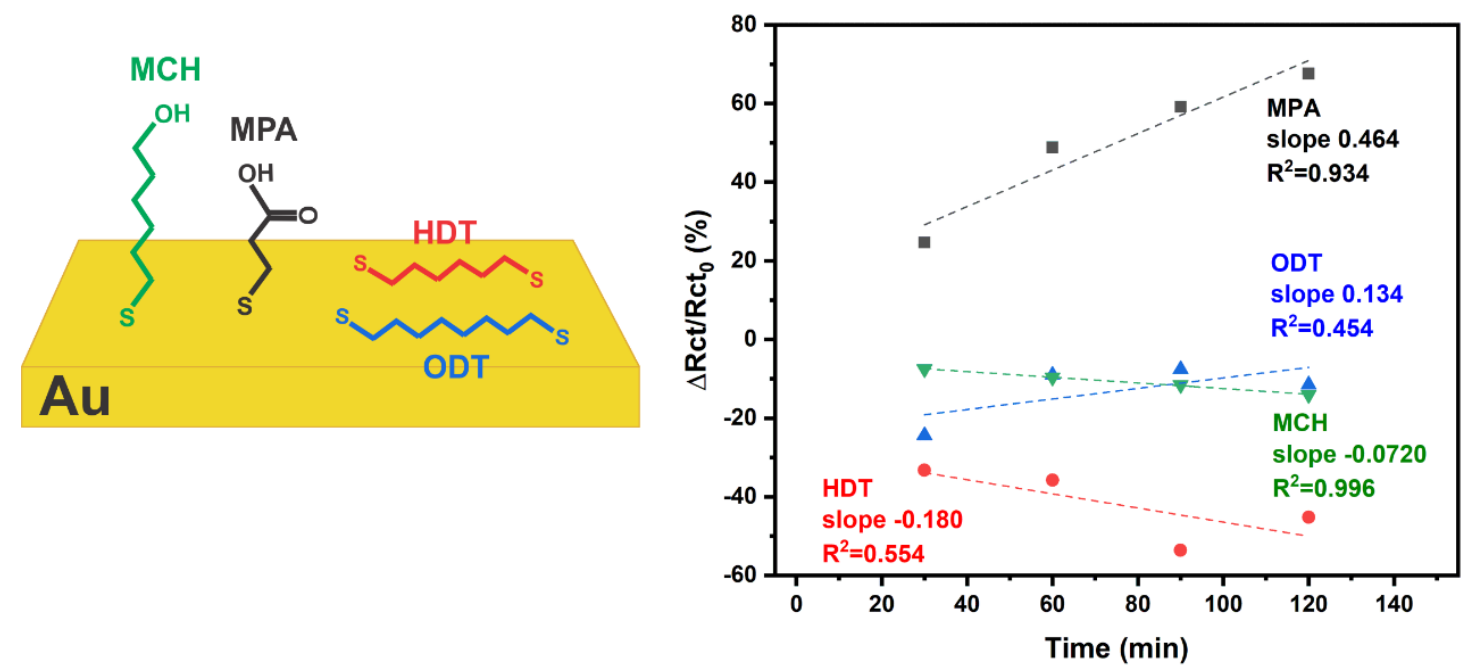

Figure 5.5. Schematic representation of the fixation of four different blocking agents MCH, MPA, HDT, and ODT on a gold electrode surface. Relative electron transfer resistance is plotted in function of the time. Each measurement is related to a BWB incubation.

A protocol using the co-incubation of thiolated aptamer and $\mathrm{MCH}$ molecule, which showed promising results concerning the organization and the stability of the SAM formed, will be explored in the next paragraph.

\section{The co-incubation of thiolated aptamer and $\mathrm{MCH}$ generates a stable and low aptamer density SAM}

The co-immobilization protocol has already been studied for creating more ordered SAMs. ${ }^{225}$, ${ }^{238}$ As demonstrated in Figure 5.5, a pure MCH SAM exhibits no signal drift related to buffer incubation. In comparison to a DNA strand, the MCH molecule is short and rigid. The main interaction occurring between the gold surface and the $\mathrm{MCH}$ molecule is the chemisorption between the sulfur group and gold atoms. The interactions between the gold surface and the 
rest of the $\mathrm{MCH}$ chain, composed of carbon and hydrogen atoms, are negligible. ${ }^{82,86}$ The expected SAM formed is, therefore, highly ordered and well-packed.

In Figure 5.6, the co-immobilization protocol consists first, in the incubation of a specific ratio of thiolated aptamers and $\mathrm{MCH}$ molecules, then, in a separated incubation of $1 \mathrm{mM} \mathrm{MCH}$.

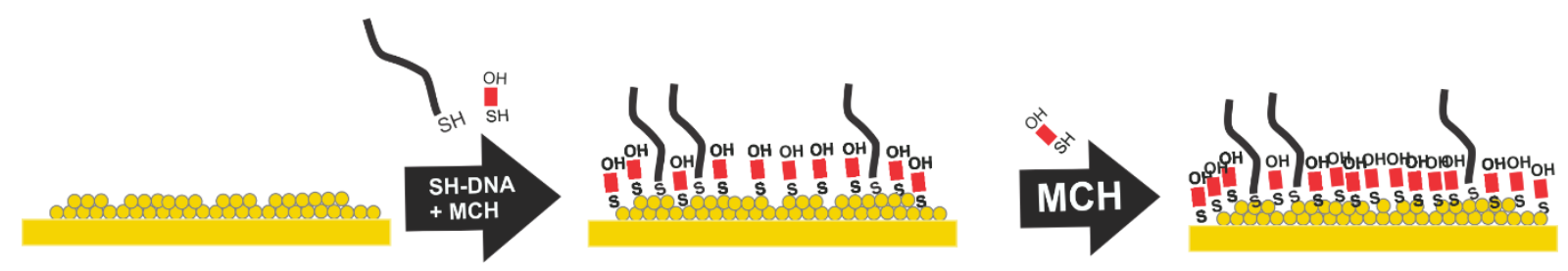

Figure 5.6. Schematic representation of the co-incubation protocol of thiolated aptamer and $\mathrm{MCH}$ molecule for the SAM formation on gold electrode surface.

As observed in Figure 5.7B, fouraptamer/ $\mathrm{MCH}$ ratios were tested and the ratio $1 / 100$ (aptamer/MCH) affords the highest aptamer density among the co-immobilization protocol ratios monitored. It is in line with the study done by Aliakbarinodehi et al. ${ }^{238}$. As a consequence, the results observed, in Figure 5.7A, of the electron transfer resistance in the function of the time are related to the ratio $1 \mu \mathrm{M} / 100 \mu \mathrm{M}$ (aptamer/MCH concentration).

Each ratio tested shows a great SAM stability in the buffer, as a consequence, we can hypothesize that a highly ordered SAM is formed with the use of the co-incubation protocol. In addition to the SAM stability, the aptamer density decreases significantly for each ratio tested in comparison to the aptamer density found with the thiolated two-step protocol in Figure 5.4.

The decrease of the aptamer density is due to the competition of fixation on the gold surface between the thiolated aptamer and the $\mathrm{MCH}$ molecule at the first incubation. With the reasons cited previously (short, rigid, and few physisorption interactions of the chain), the MCH molecule has more facility to interact with the gold surface and, thus, a higher MCH density occurs in comparison to the aptamer density. As a consequence, a major drawback of this protocol is the significant decrease of the aptamer density on the gold electrode surface.

As shown in Chapter 3, the binding event between the Aptamer-2 and the methamphetamine is entropically driven and no major structural change for the aptamer is generated. Therefore, as explained in Figure 5.2, decreasing the aptamer density is not the best strategy to adopt for the Aptamer-2 - Meth specific case. Consequently, as shown in Figure 5.7A, no Rct change 
appears between the BWB and the target addition. In conclusion, the SAM stability has been greatly improved, however, the aptamer density remains too low to be able to detect any specific binding event on the aptamer surface.
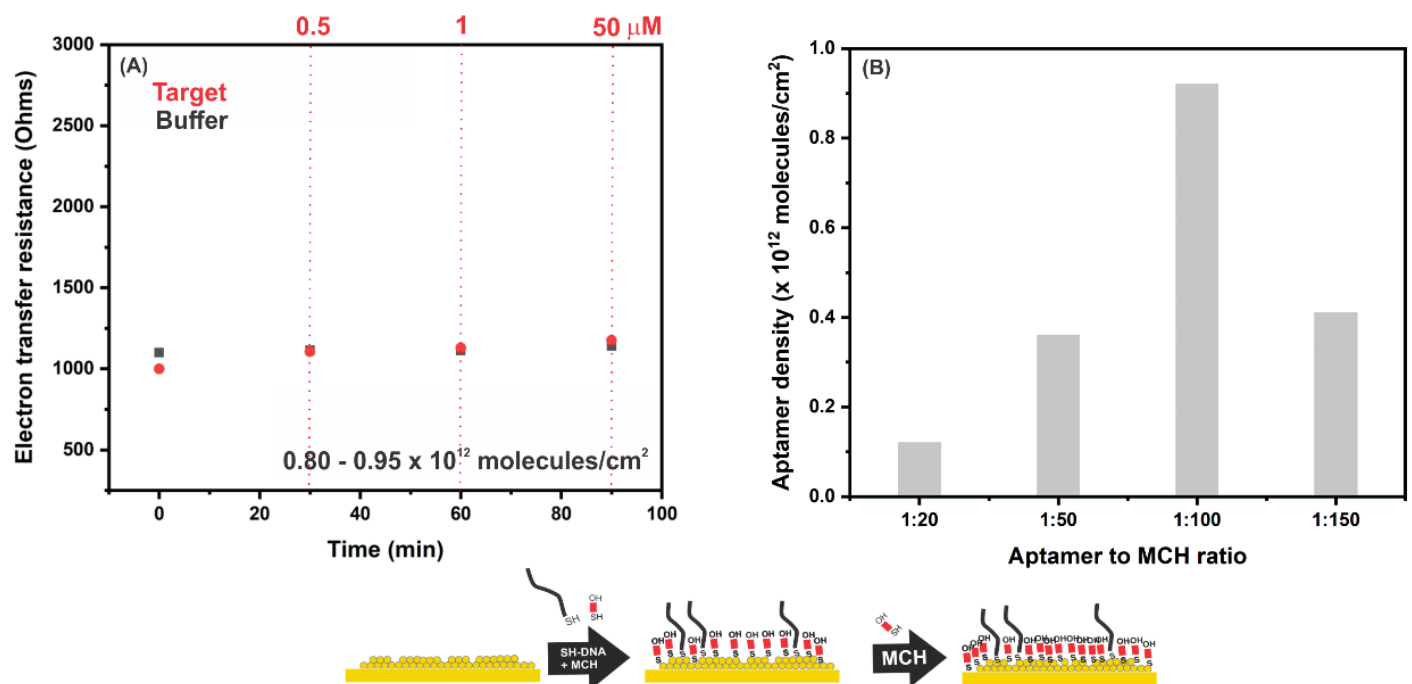

Figure 5.7. (A) EIS results for the co-incubation protocol with the 75 mer aptamer $-1 \mu \mathrm{M}$ aptamer incubated/100 $\mu \mathrm{M} \mathrm{MCH}$. (B) Chronocoulometry results for different ratio of aptamer/MCH.

In the next section, an optimization, combining truncation and different thiol aptamer concentrations incubated, of the two-step protocol using thiolated aptamer and MCH will be explored.

\section{The SAM affords a better stability upon higher thiolated aptamer concentration incubated}

As discussed in Xu et al. ${ }^{234}$, the self-assembly of thiols onto gold can be accelerated by using catalysts, by increasing the temperature of reaction, or by increasing the concentration of reactants. ${ }^{221,234,239}$ In our case, using catalysts is not suitable for the desired final system, indeed, they are molecules that may interact with DNA strands, and they must be removed from the system. As discussed previously in $\mathrm{Xu}$ et al. $^{120}$, the increase of the reaction temperature is not appropriate for the production of a highly ordered SAM. By deduction, the initial thiolated aptamer concentration incubated parameter will be explored for trying to optimize the kinetics of fixation of the DNA strands on the gold surface. 
In the case of the original Aptamer-2-75mer, we can observe in Figure 5.8A that the concentration of the incubated thiol aptamer influences the SAM stability formed. The stability is enhanced greatly for the use of $5 \mu \mathrm{M}$ and $10 \mu \mathrm{M}$ thiolated aptamer concentrations incubated. After $30 \mathrm{~min}$ of incubation in BWB, the electron transfer resistance remains constant in comparison to the $R c t$ values found for the $1 \mu \mathrm{M}$ Aptamer-2 $75 \mathrm{mer}$ conditions observed in Figure 5.4. As explained in $\mathrm{Xu}$ et al. ${ }^{234}$, the high concentration environment can create a spatial confinement environment at the gold surface, and thus the process of SAM formation can be faster in comparison to a more dilute aptamer concentration environment. As a result, the homogeneity of the SAM will be higher than for lower thiolated aptamer concentration incubated conditions.

The second observation is that a smaller Rct is observed in comparison to the $1 \mu \mathrm{M}$ protocol (Figure 5.4). The decrease of $R c t$ also occurs from 5 to $10 \mu \mathrm{M}$ thiolated aptamer concentration incubated. By increasing the aptamer concentration incubated a steric hindrance effect could occur inter-75mer strands and hence, influences the efficiency of the fixation of the aptamer. As a consequence of the higher thiolated aptamer concentration incubated, the homogeneity can be higher with fewer aptamer strands fixed on the gold electrode surface.
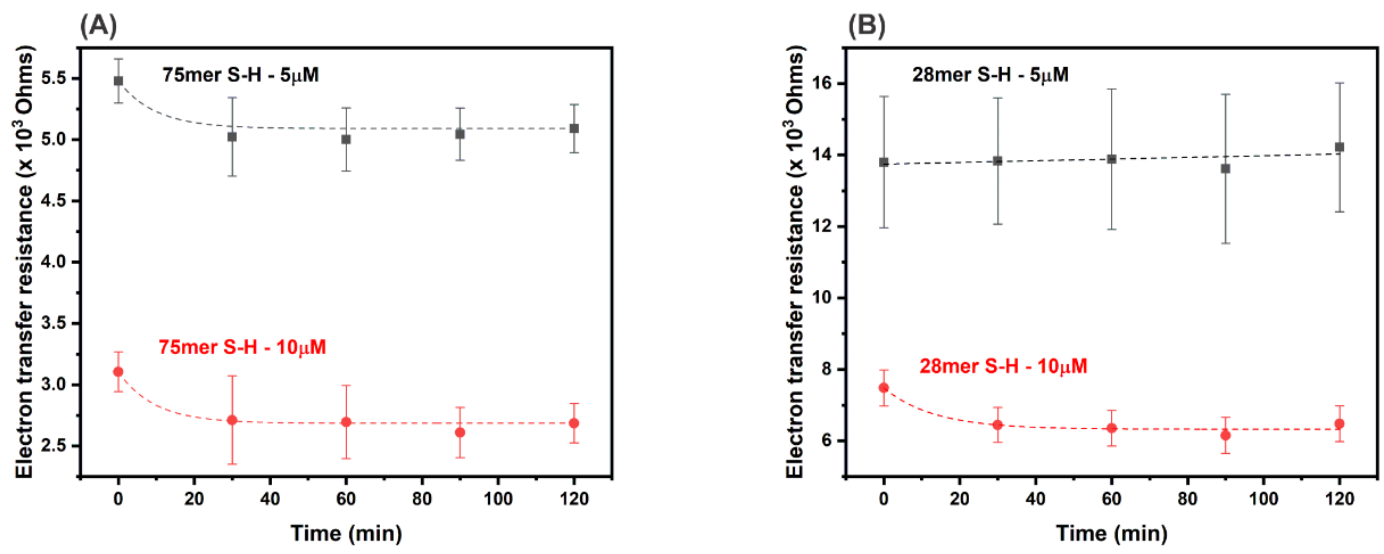

Figure 5.8. (A) Stability study for the thiolated 75 mer aptamer incubated at $5 \mu \mathrm{M}$ and $10 \mu \mathrm{M}$. (B) Stability study for the thiolated 28 mer aptamer incubated at $5 \mu \mathrm{M}$ and $10 \mu \mathrm{M}$. The two graphs represent the Rct in the function of the time for consecutive BWB. 


\section{Truncation of the Aptamer-2 75mer has an impact on the aptamer fixation}

The effect of the aptamer length on the SAM formation will be studied by using and comparing a truncated aptamer 28 nucleotides long (28mer), which conserves the binding region observed in Chapter 3, with the original 75mer aptamer. By shortening the aptamer from 75 to 28 nucleotides, the SAM stability still occurs (Figure 5.8B). An enhancement of the Rct value in comparison to the $75 \mathrm{mer}$ is visible. For $5 \mu \mathrm{M} 28 \mathrm{mer}$ aptamer incubated, a value of 13,802 \pm $1,836 \mathrm{Ohms}$ is observable in contrast to the $75 \mathrm{mer}$ which shows a value of $5,481 \pm 181 \mathrm{Ohms}$ at the same concentration. As explained in Liu et al. ${ }^{59}$, a DNA strand has a significant affinity with the gold surface. Meaningful physisorption between the aptamer strand and the gold surface could be one of the main drawbacks for the creation of homogeneous SAM and for having an effective reaction of chemisorption between the sulfur group and the gold surface. The truncation of the aptamer reduces the physisorption affinity between the DNA strand and the gold surface; therefore, we can suppose that the fixation will be more efficient for a $28 \mathrm{mer}$ aptamer in comparison with a 75mer as observed in Figure 5.9.

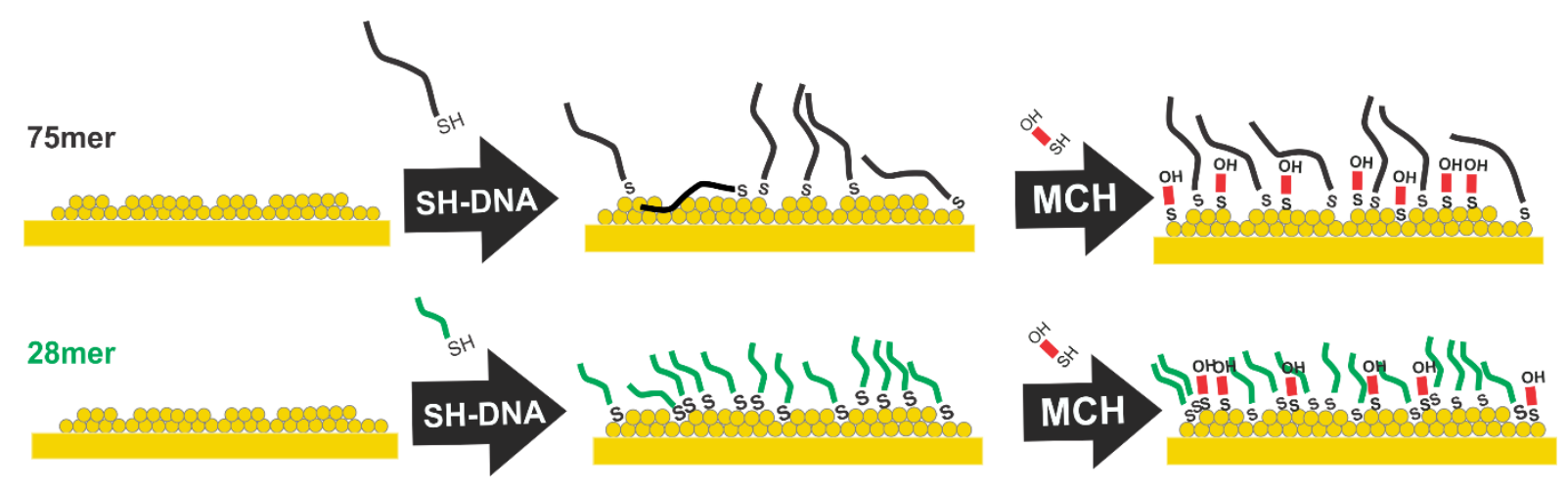

Figure 5.9. Schematic illustration of the effect on the SAM fixation for a truncated aptamer (28mer). Less inter-DNA strand interactions occur, more DNA strand can be fixed on the gold electrode surface.

In addition, the same trend of $R c t$ decrease observed with the $75 \mathrm{mer}$ aptamer arises by increasing the aptamer concentration incubated with the 28 mer aptamer. At $5 \mu \mathrm{M}$ the value is $13,802 \pm 1,836 \mathrm{Ohms}$ and at $10 \mu \mathrm{M}$, the value decreases to 7,482 $\pm 500 \mathrm{Ohms}$. To have an insight view about the SAM formation dependency towards the thiolated aptamer concentration incubated and the aptamer length; the aptamer density and the electron transfer resistance were plotted in the function of the aptamer concentration incubated. (Figure 5.10). 


\section{Aptamer density on the gold electrode remains limited and dependent on the aptamer length}

For the 75 mer version of the aptamer, in Figure 5.10A, the electron transfer resistance decreases upon the aptamer concentration increase as previously found, however, the aptamer density remains constant for the four aptamer concentrations incubated examined. The hypothesis stated previously about less aptamer fixed at high thiolated aptamer concentrations incubated is rejected. A new hypothesis for that effect has to be proposed.

The plausible explanation for the Rct decrease upon the increase of the aptamer concentration incubated is expressed as follows: at higher thiolated aptamer concentration, more physisorbed DNA strands are on the gold electrode surface, however, due to the crowding environment the chemisorption between the sulfur group and a gold atom doesn't occur for each of them. By being physisorbed, those DNA strands prevent the chemisorption of the MCH blocking agent. As a result, the aptamer density is limited to a maximum value, and fewer $\mathrm{MCH}$ molecules are fixed on the gold electrode surface. Consequently, more bare gold surfaces appear and the electron transfer resistance decreases.

In Figure 5.10B, no correlation between the electron transfer resistance and the aptamer density is observable. This "no correlation" behavior found may represent the inconsistency into the SAM formation for the 75 mer thiolated aptamer. Concerning the 28 mer aptamer, in Figure 5.10C, similar values are observable for the $0.5,1$, and $5 \mu \mathrm{M}$ aptamer concentrations incubated. The aptamer density and the electron transfer resistance remain constant for these three concentrations.

As already discussed in the previous section, the electron transfer resistance is more significant for the 28 mer version in comparison with the 75 mer. With Figure 5.10C, we can observe that the aptamer density is more significant for the 28 mer too. Indeed, the value vary around $4.0 \mathrm{x}$ $10^{12}$ molecules $/ \mathrm{cm}^{2}$ for the $28 \mathrm{mer}$ and around $1.6 \times 10^{12}$ molecules $/ \mathrm{cm}^{2}$ for the $75 \mathrm{mer}$. The hypothesis stated previously is confirmed, shortening the aptamer enhances the aptamer fixation on the gold electrode surface. The interaction of fixation would be mainly between the sulfur moiety and the gold surface, by truncating the aptamer the tail-surface and tail-tail interactions will be minimized. ${ }^{84,121,221}$ Concerning the $10 \mu \mathrm{M} 28$ mer aptamer concentration, as observed in Figure 5.10C, the aptamer density value increases, and the electron transfer resistance value decreases drastically. Similar behavior is observed and explained previously with the 75 mer. 
As both values don't increase proportionally, we can still think that a lot of aptamers are physisorbed and prevent the MCH fixation on the gold electrode surface. In the lower range of aptamer concentration incubated, the $\mathrm{MCH}$ molecule can remove the aptamers strands physisorbed on the gold electrode surface, and hence, the only aptamers fixed on the gold electrode are chemisorbed. In the case of high aptamer concentration incubated, too many aptamer strands are physisorbed on the surface and the $\mathrm{MCH}$ molecule cannot remove all of them. Thus, the aptamer density is higher (more ruthenium molecules during the chronocoulometry measurement can interact with the negative phosphate backbone of the DNA strands), nevertheless the SAM is significantly heterogeneous.

The graph of the Rct in the function of the aptamer density in Figure 5.10D shows a better correlation between these two parameters in comparison to the $75 \mathrm{mer}$. The correlation is better, however, the Rct value decreases proportionally to the increase of the aptamer density, this could be viewed as a not ideal trend for the creation of a highly ordered SAM. 


\section{$75 \mathrm{mer}$ - thiol protocol}
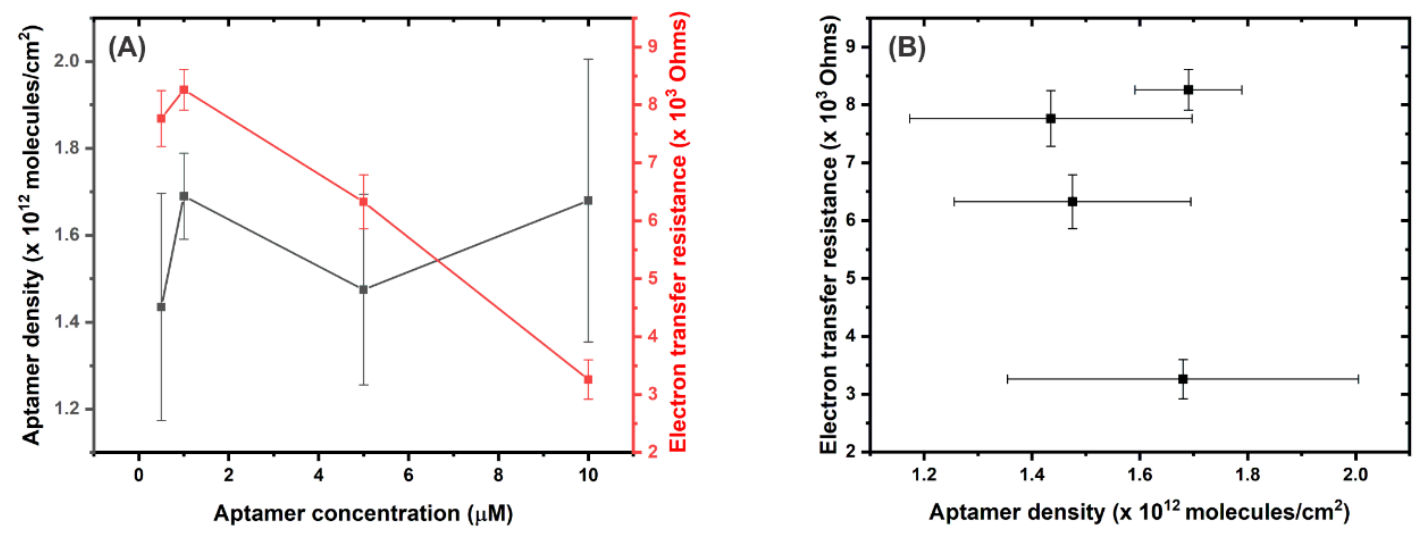

\section{8mer - thiol protocol}
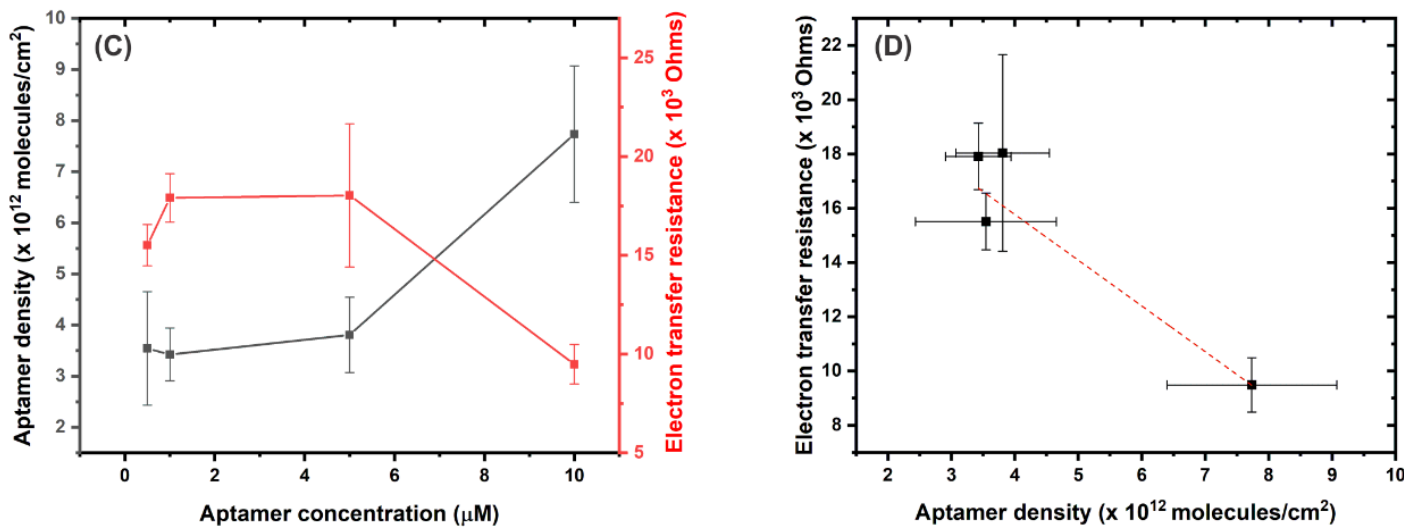

Figure 5.10. Investigation of the aptamer density and the electron transfer resistance for different thiolated aptamer incubated concentrations. (A) and (B) represent the 75 mer thiolated aptamer. (C) and (D) represent the 28 mer thiolated aptamer.

After obtaining a stable EIS signal with the high thiolated aptamer concentrations incubated (5 and $10 \mu \mathrm{M}$ ) for 75 and 28mer Aptamer-2, a Meth addition will be investigated in the next section.

\section{No Rct signal change appears for the thiolated 75 and 28 mer aptamers upon Meth addition}

Finally, target incubation experiments have been done to observe any change in the Rct value upon the addition of methamphetamine for the high 75 and 28 mer thiolated aptamer concentrations incubated. The target incubation consists of two methamphetamine concentrations in the nanomolar range $(500 \mathrm{nM})$ and the micromolar range $(50 \mu \mathrm{M})$. These 
values have been chosen in correlation with the $K_{D}$ value found with the SG assay and ITC measurement in Chapter 3.

As observed in Figure 5.11, no significant change of Rct is visible for both versions of the thiolated aptamer. A hypothetic explanation is that the SAM formed remains heterogeneous. As shown and stated previously, the aptamer density is limited due to the physisorbed DNA strands. Indeed, the maximization of the aptamer density and the electron transfer resistance is not achievable with the two-step thiolated protocol.
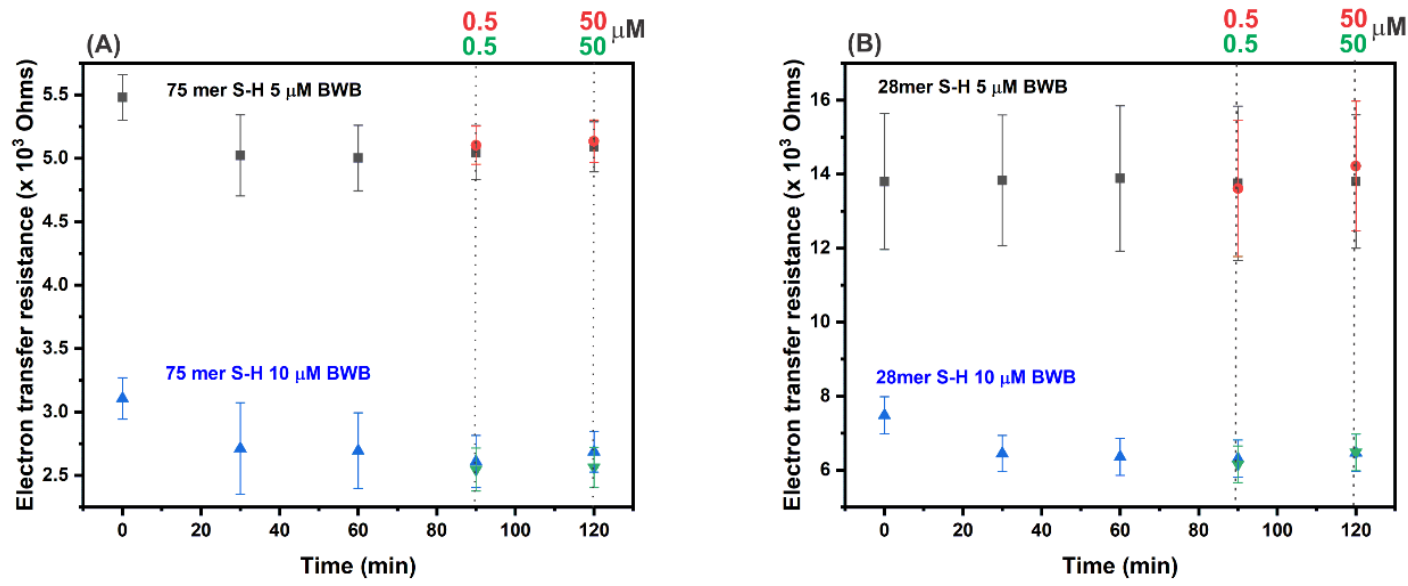

Figure 5.11. Investigation of the target incubation at $500 \mathrm{nM}$ and $50 \mu \mathrm{M}$ methamphetamine. (A) represents the 75 mer thiolated aptamer at $5 \mu \mathrm{M}$ and $10 \mu \mathrm{M}$ incubated. (B) represents the 28 mer thiolated aptamer at $5 \mu \mathrm{M}$ and $10 \mu \mathrm{M}$.

The aptamer density cannot be increased with the two-step protocol using thiolated aptamer. A new protocol must be developed to enable the maximization of the aptamer density. This new method will be presented in the next section. 


\subsubsection{Disulfide aptamer strategy}

To bypass the limited aptamer fixation observed previously with the thiolated aptamers, the idea of using disulfide aptamers for the fixation of two aptamers strands simultaneously will be explored. Related to the SAM formation, the use of a disulfide aptamer protocol has never been examined. Mehennaoui et al. ${ }^{240}$ used a disulfide molecule combing a $\mathrm{MCH}$ molecule on one side and an aptamer on the other side for SAM preparation. However, they didn't mention why they chose this approach.

Numerous studies already investigated the use of disulfide alkyl molecules for SAM arrangement. ${ }^{83,219,221,241}$ Rouhana et al. ${ }^{221}$ concluded that the kinetics of SAM formation between the thiol and the disulfide approach is quasi similar. The study investigated disulfide short alkyl molecules fixation on a gold surface. The use of a disulfide molecule composed of long DNA strands may react differently with a gold surface due to the size, flexibility, and chemical composition of the DNA strand. Furthermore, the simultaneous fixation of two DNA strands with the disulfide approach instead of the fixation of one DNA strand with the thiolated approach can have a significant impact on the stability and aptamer density of the formed SAM (Figure 5.12). The next sections will investigate the formation of disulfide aptamers, and the consequence of using a dimer aptamer for a SAM formation.
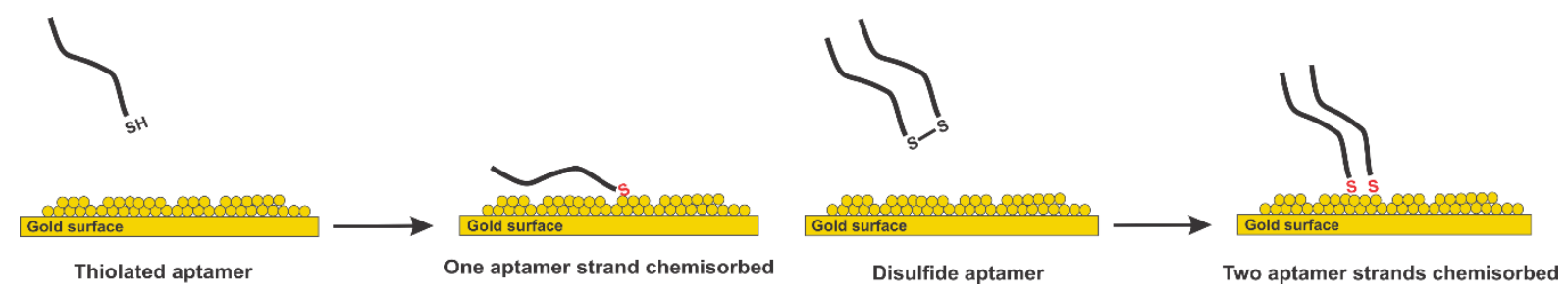

Figure 5.12. Schematic representation of the thiolated aptamer fixation approach and the disulfide aptamer fixation approach.

\section{DMSO affords the mild oxidation of thiol aptamer in disulfide aptamer}

To form a disulfide aptamer from a thiol aptamer stock, mild oxidation of the thiol group must be done. The use of strong oxidizers such as peroxide hydrogen or halogens $\left(\mathrm{Cl}_{2}\right.$ or $\left.\mathrm{F}_{2}\right)$ must be avoided as they may denature the DNA strand. Some mild oxidizers suitable for use in a biological environment were found: atmospheric oxygen, ${ }^{242,}{ }^{243}$ industrial grade compressed oxygen with $99.5 \%$ purity, ${ }^{243}$ and DMSO. ${ }^{244-248}$ 
To monitor the oxidation of a thiol group in a disulfide group, Ellman's reagent was used ${ }^{249}$, 250. The concentration of the thiol compound in solution is proportional to the yellow color produced by the formation of the TNB molecule, resulting in the reaction of the Ellman's reagent (DTNB) with the thiol molecule of interest. As a consequence, the thiol concentration in the solution can be followed via UV-visible spectroscopy at $412 \mathrm{~nm}$. Because of the limit of detection of Ellman's experiment, a high concentration of thiol molecules must be used. That's why acetylcysteine (or cysteine) was used as a standard thiol molecule instead of the thiolated aptamer. Its reaction with Ellman's reagent is shown in Figure 5.13.

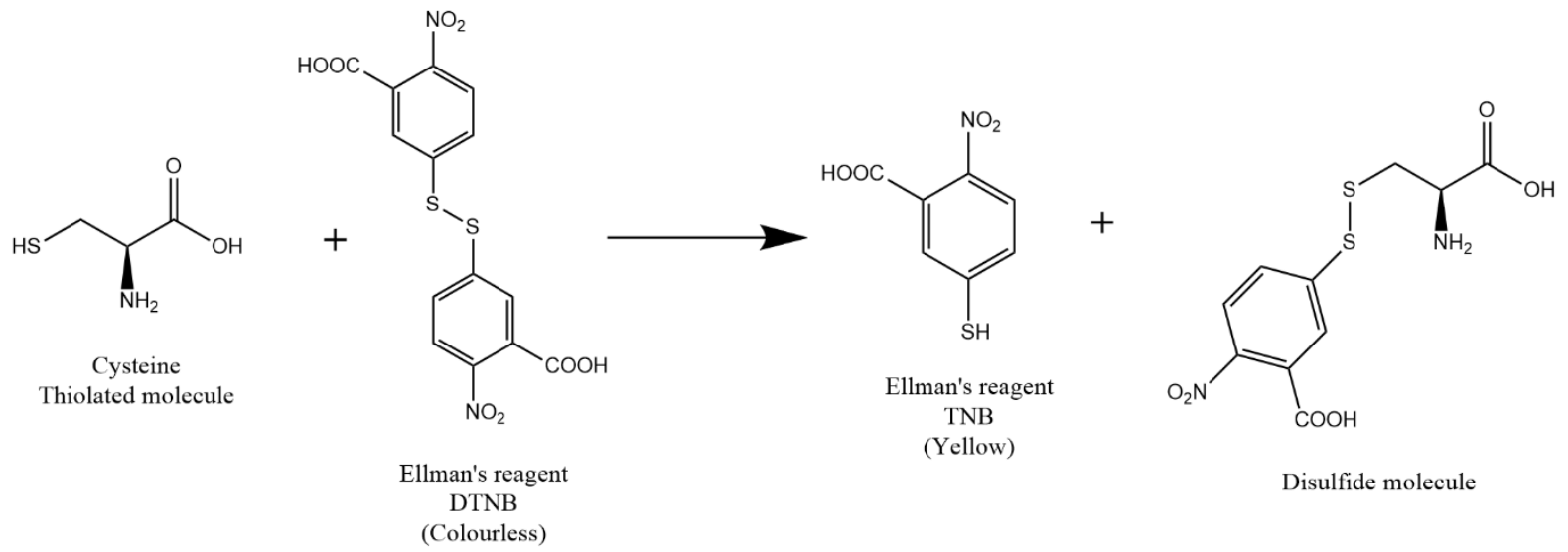

Figure 5.13. Ellman's experiment overall equation for the reaction of the acetylcysteine with the Ellman's reagent (DTNB, colourless). It forms a disulfide molecule (mix of the cysteine and DTNB molecule, colourless) and the TNB molecule (yellow color).

A calibration curve was generated for the acetylcysteine molecule. As shown in Figure A.5, the absorbance at $412 \mathrm{~nm}$ increases with the increase of the acetylcysteine concentration. Indeed, more TNB molecules are produced when the concentration of thiol molecules increases. An absorbance saturation is notified at $500 \mu \mathrm{M}$ cysteine (Figure A.5). As a consequence, the experiments of thiol oxidation were undertaken with an initial cysteine concentration of $500 \mu \mathrm{M}$. Three different protocols of oxidation were tested: the atmospheric oxidation of the cysteine by leaving the solution uncovered, direct injection of oxygen in the cysteine solution by degassing the solution with industrial grade compressed oxygen, and 20 $\%$ of pure DMSO addition to the final volume of the cysteine solution.

The results of the oxidation experiments are represented in Figure 5.14. No data related to the direct bubbling of $\mathrm{O}_{2}$ into the solution have been acquired due to the resulting evaporation of the cysteine solution.The two other conditions (atmospheric oxygen and $20 \%$ DMSO) have 
been compared in Figure 5.14A. After 30 min of reaction, no significant change of the solution absorbance occurs $(0.7 \%$ of S-H conversion into S-S for $20 \%$ DMSO, inconsistent increase for the atmospheric oxygen). After $3 \mathrm{~h}$ of reaction, a decrease of the absorbance for the $20 \%$ DMSO solution is observable (18\% of S-H conversion into S-S for $20 \%$ DMSO, inconsistent increase for the atmospheric oxygen). The trend of the decreasing absorbance for the $20 \%$ DMSO solution continues after $5 \mathrm{~h}(68 \% \mathrm{~S}-\mathrm{H}$ conversion into S-S for $20 \%$ DMSO, $2.7 \%$ conversion for the atmospheric oxygen) then an absorbance close to 0 is readable after $22 \mathrm{~h}$ of oxidation reaction with the use of $20 \%$ DMSO (103\% of S-H conversion into S-S for $20 \%$ DMSO, 6\% of conversion for the atmospheric oxygen) (Figure 5.14B). The atmospheric oxidation shows a negligible absorbance decrease after $22 \mathrm{~h}$ of reaction and, therefore, a negligible conversion of thiol into disulfide.

The absorbance decrease can be explained as follows: because of the oxidation of the thiol groups in disulfide groups belonging to the cysteine, the reaction of the thiol groups with the Ellman's reagent to form the TNB final yellow product of the Ellman's reaction will be prevented. The mechanism of the oxidation of thiol molecule with the use of DMSO is presented in Figure A.6. ${ }^{245}$
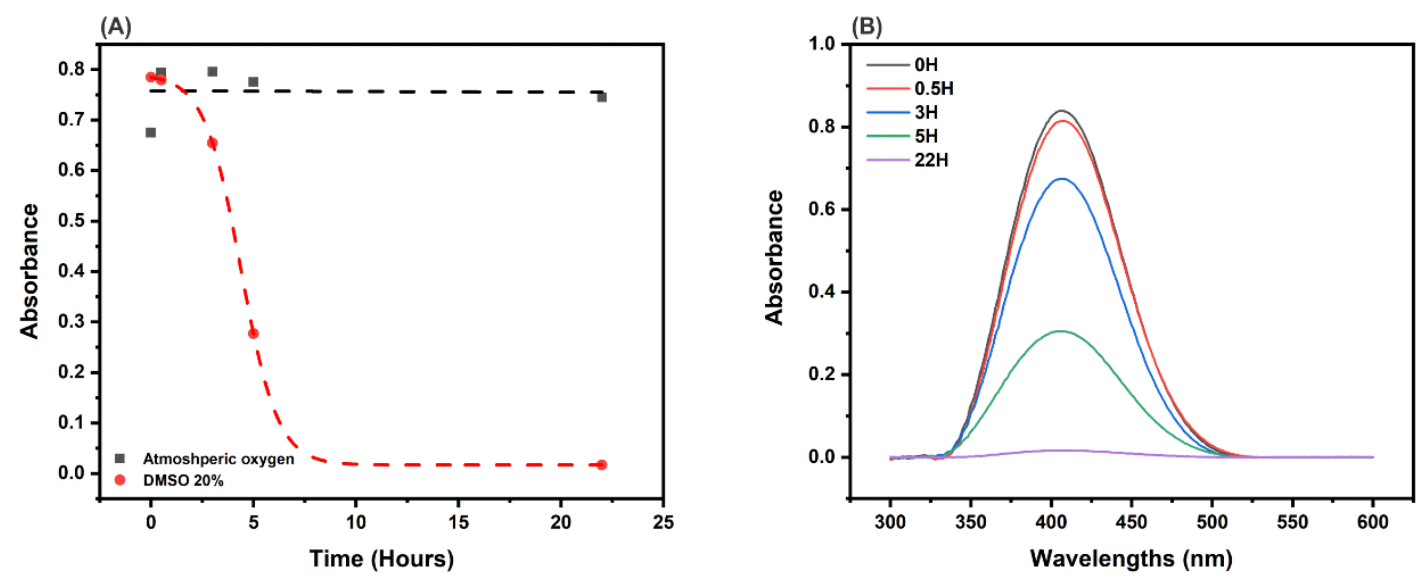

Figure 5.14. Mild oxidation experiment of the cysteine thiol group into disulfide. (A) Representation of the absorbance at $412 \mathrm{~nm}$ in the function of the time. (B) Raw data for the decrease of absorbance at $412 \mathrm{~nm}$ for the cysteine - $20 \%$ DMSO solution. 
As a result, the protocol of oxidation of the thiolated DNA strands using $20 \%$ DMSO will be used for the rest of the investigation. $5 \mathrm{~h}$ oxidation time in the presence of $20 \%$ DMSO will be used as the standard protocol for the following experiments. $5 \mathrm{~h}$ is used for a practical purpose, and because more than $50 \%$ of the initial thiol groups are converted to disulfide groups after $5 \mathrm{~h}(68 \%$ of conversion $)$.

\section{The disulfide aptamer protocol produces a stable and high aptamer density SAM}

The single thiolated aptamer is converted to a disulfide dimer aptamer with the use of the 20 \% DMSO protocol as shown in Figure 5.15.

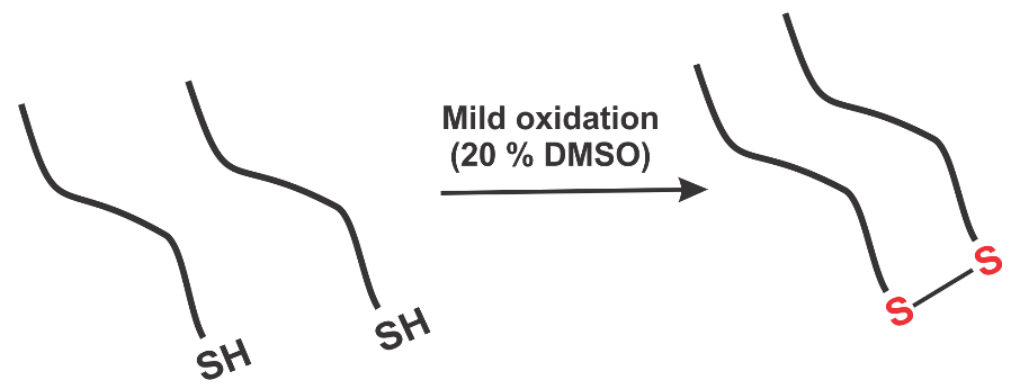

Figure 5.15. Conversion of two thiolated aptamer into a disulfide dimer aptamer.

The first investigation achieved concerns the stability of the SAM formed by using the $20 \%$ DMSO protocol with the thiolated 75 and 28mer aptamers (75mer S-S and 28mer S-S protocols). As observed in Figure 5.16A, the SAM formed for the thiolated 75mer treated with $20 \%$ DMSO is stable, and the major difference with the thiolated 75mer untreated is the large initial electron transfer resistance occurring. Indeed, the initial $R c t$ value for the $5 \mu \mathrm{M}$ thiolated 75 mer protocol is $5,481 \pm 180 \mathrm{Ohms}$ and the initial value for the $5 \mu \mathrm{M}$ thiolated $75 \mathrm{mer}$ treated with $20 \%$ DMSO is $69,358 \pm 2,570$ Ohms. The Rct value is approximately 13 times higher for the $20 \%$ DMSO-treated thiolated aptamer.

The great stability and the important initial electron transfer resistance are observed as well for the 28mer aptamer treated with $20 \%$ DMSO (Figure 5.16B).However, only one observation is specifically related to the $28 \mathrm{mer} \mathrm{S}-\mathrm{S}$ protocol, the initial electron transfer resistance is more important for $10 \mu \mathrm{M}$ than for $5 \mu \mathrm{M}$ concentration of aptamer incubated. Indeed, for all previous experiments related to the $75 \mathrm{mer} \mathrm{S-H,} 28 \mathrm{mer} \mathrm{S-H}$, and $75 \mathrm{mer} \mathrm{S}-\mathrm{S}$, the $R c t$ value decreases drastically from 5 to $10 \mu \mathrm{M}$ concentration of aptamer incubated. This observation has been attributed to the fact that too many DNA strands, nonspecifically adsorbed to the electrode 
surface, prevent the MCH molecules to interact with the gold surface and, thus, create a $R c t$ decrease. For the 28mer S-S protocol, this assumption is not plausible and may be due to the fixation of the two short aptamers strands. An aptamer density investigation must be done to conclude about the characteristics of the formed SAM with the disulfide protocol.
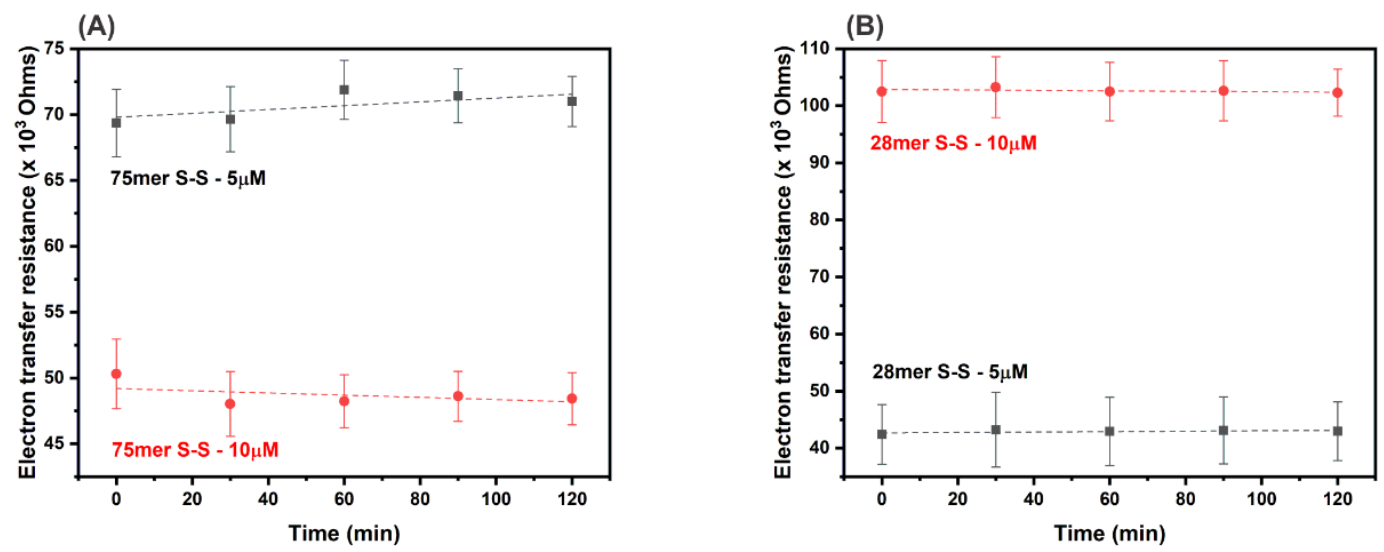

Figure 5.16. (A) Stability study for the disulfide 75 mer aptamer incubated at $5 \mu \mathrm{M}$ and $10 \mu \mathrm{M}$. (B) Stability study for the disulfide 28 mer aptamer incubated at $5 \mu \mathrm{M}$ and $10 \mu \mathrm{M}$. The two graphs represent the $R c t$ in the function of the time for consecutive BWB incubations.

From Figure 5.17A and Figure 5.17C, the electron transfer resistance and the aptamer density in the function of the concentration of the aptamer incubated are observed for the disulfide protocol.Concerning the 75mer S-S protocol, in Figure 5.17A, the Rct and the aptamer density are more important than with the thiol protocol. For the 75 mer disulfide aptamer, the aptamer density and the Rct increase until reaching a maximum at $5 \mu \mathrm{M}$ concentration of aptamer incubated. The maximum $R c t$ value is $83,971 \pm 8,031 \mathrm{Ohms}$ and the maximum aptamer density value is $4.8( \pm 0.1) \times 10^{12}$ molecules $/ \mathrm{cm}^{2}$. Then a proportional decrease of the Rct and the aptamer density occurs at $10 \mu \mathrm{M}$.

A good correlation for the disulfide protocol, which is not observed for the thiol protocol, occurs between the aptamer density and the Rct (Figure 5.17B). The Rct increases proportionally to the aptamer density which may reflect a good control on the SAM formation. In addition, at $10 \mu \mathrm{M}$, the Rct and the aptamer density decrease proportionally. This trend is contrary to the one observed for the thiolated aptamer and shows the contrast between the two protocols. The 28 mer aptamer has been used with the disulfide protocol in the next paragraph to observe if a correlation between the Rct and the aptamer density is also observed. 
In Figure 5.17C, the same observations are visible for the $28 \mathrm{mer} \mathrm{S}-\mathrm{S}$ protocol in comparison to the $75 \mathrm{mer} \mathrm{S}-\mathrm{S}$ protocol. An increase, in comparison to the thiol protocol, in the aptamer density as well as in the Rct is shown. However, for the $28 \mathrm{mer} \mathrm{S}-\mathrm{S}$ protocol, by increasing the aptamer concentration from 5 to $10 \mu \mathrm{M}$, the $R c t$ and the aptamer density still increase. A maximum aptamer density of $12( \pm 3) \times 10^{12}$ molecules $/ \mathrm{cm}^{2}$ is reached at $10 \mu \mathrm{M}$ aptamer concentration incubated for the $28 \mathrm{mer} \mathrm{S}-\mathrm{S}$ protocol. As shown for the $75 \mathrm{mer} \mathrm{S}-\mathrm{S}$ protocol, a clear correlation between the aptamer density and the electron transfer resistance is shown for the 28mer S-S protocol in Figure 5.17D.

$75 \mathrm{mer}$ - disulfide protocol
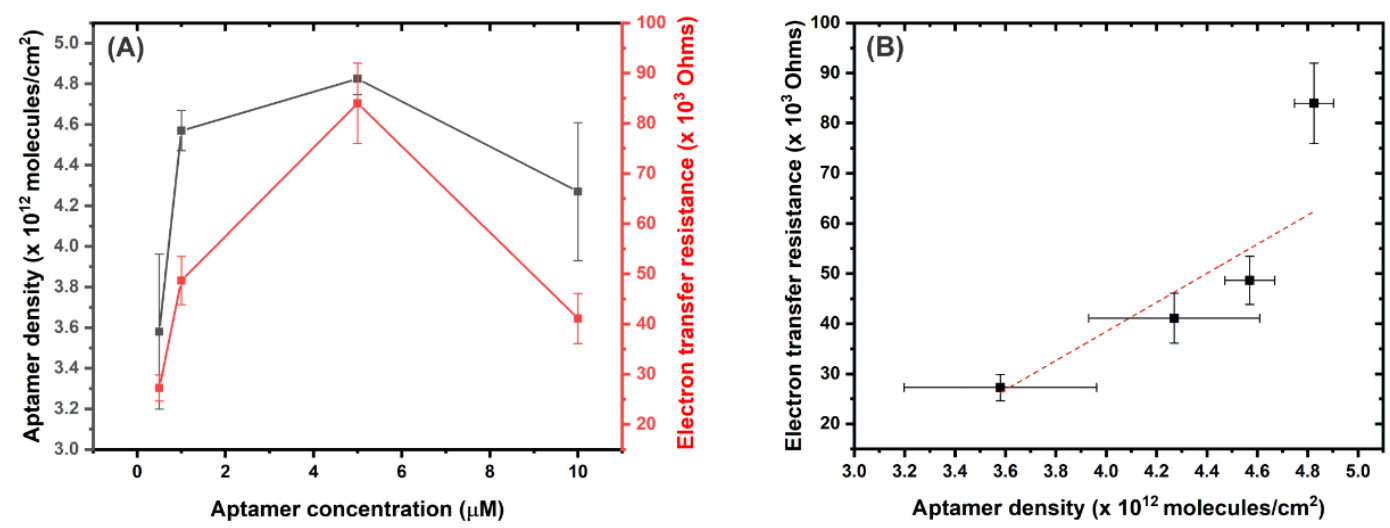

28mer - disulfide protocol
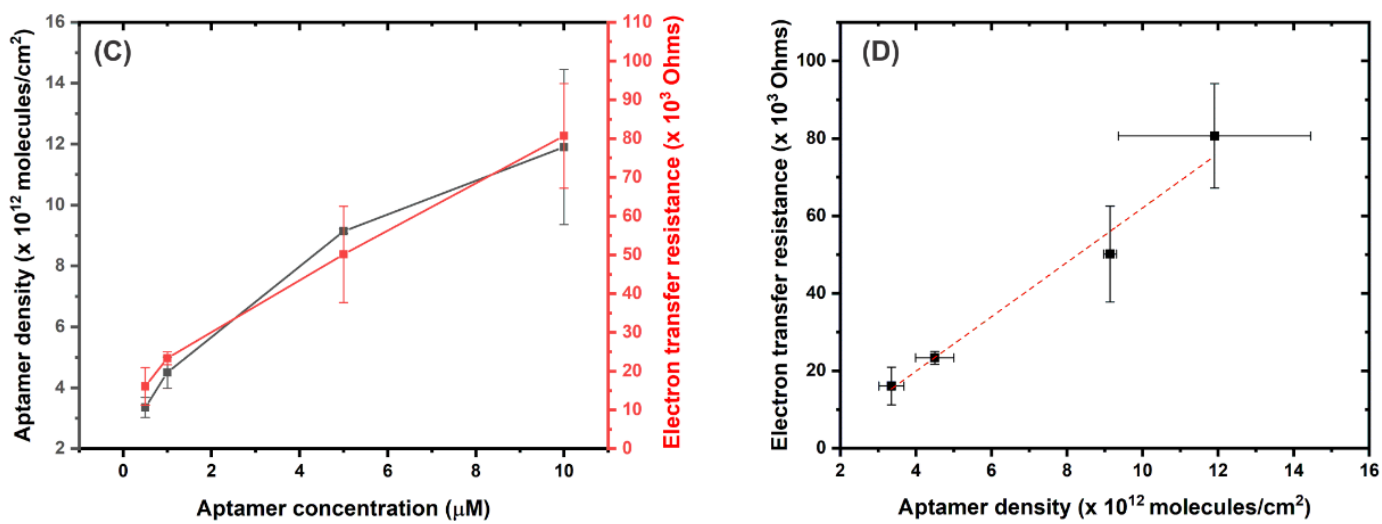

Figure 5.17. Investigation of the aptamer density and the electron transfer resistance for different disulfide aptamer incubated concentrations. (A) and (B) represent the 75 mer disulfide aptamer. (C) and (D) represent the 28 mer disulfide aptamer. 
The trend of the correlation between the Rct and the aptamer density for the S-S protocol is the inverse of the S-H protocol. Two hypotheses of SAM formation and organization can be explored for the two-step disulfide protocol: The first assumption done for the S-S protocol is that a more homogeneous SAM is formed. The conclusion is similar to the case explained in Rouhana et al. ${ }^{221}$, the S-S protocol forces the adsorbates to be into the "standing-up" position directly, bypassing the "lying-down" configuration as shown in Figure 5.18 (3a) resulting in a highly densely packed monolayer.

The second assumption done for the S-S protocol is that by fixing two aptamer strands simultaneously, the electrode surface becomes crowded and disordered as observed in Figure 5.18 (3b). However as shown from previous chronocoulometry experiments, the aptamer density is large which means that the DNA strands are covalently bound to gold. The DNA strands are not just physisorbed as seen in high aptamer concentration incubated for the twostep thiolated experiments.

In Figure 5.18, case (3) represents the hypotheses of fixation done with the S-S protocol. The step of aptamer fixation is different from the two other protocols used in this chapter (case (1) two-step protocol with thiolated aptamer and case (2) co-immobilization of aptamer/MCH protocol). The Meth addition will be explored for 5 and $10 \mu \mathrm{M}$ incubated of the 75 and $28 \mathrm{mer}$ using the disulfide protocol in the next paragraph. 


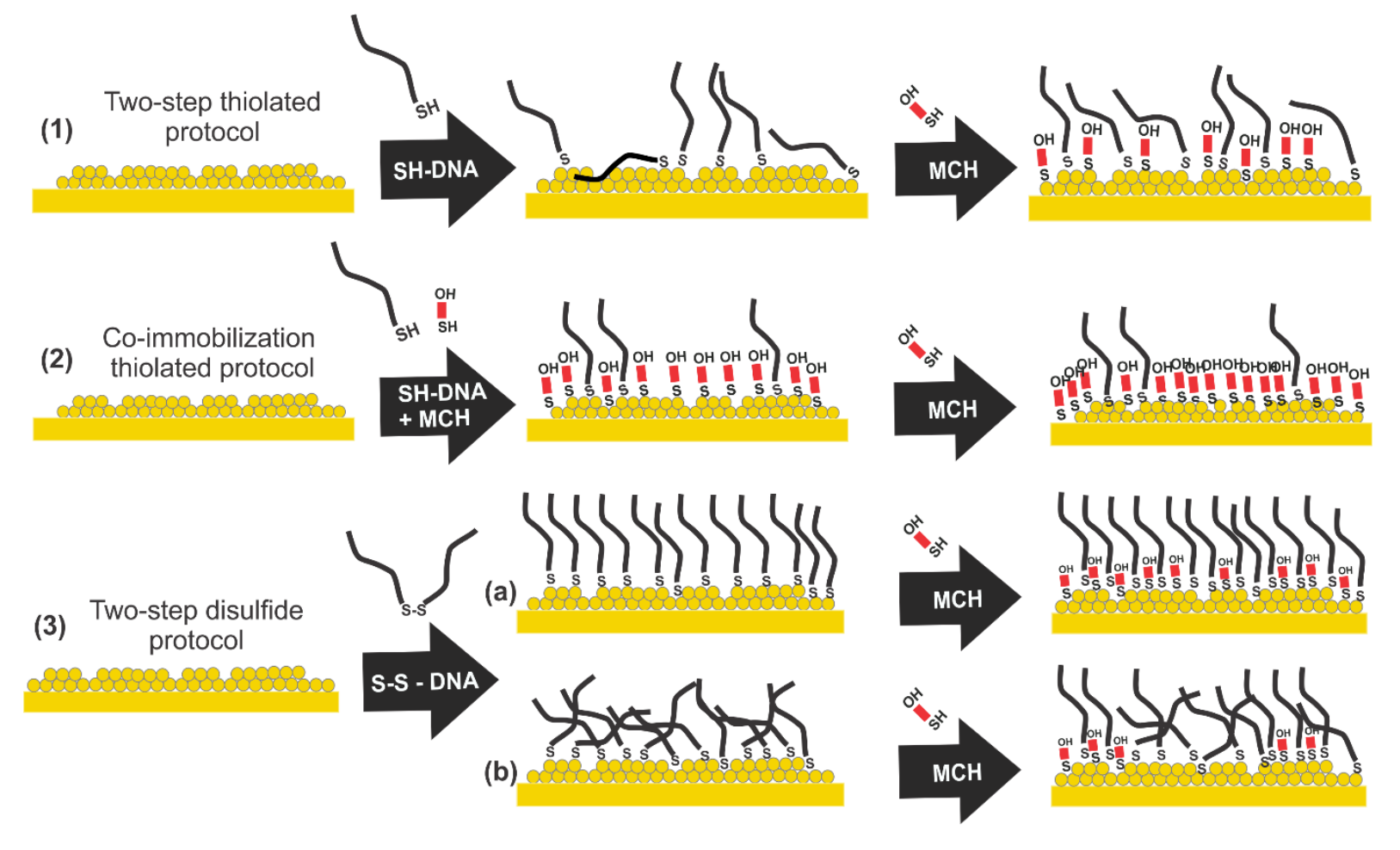

Figure 5.18. Schematic representation of the three different methods of SAM fixation tested. (1) The two-step thiolated protocol, (2) the co-incubation protocol, and (3) the two-step disulfide protocol with (a) the first ordered SAM hypothesis and (b) the disordered SAM hypothesis.

\section{A Rct signal change occurs upon Meth addition with the disulfide protocol}

Meth addition experiments have been done similarly to the thiolated aptamer experiments observed in Figure 5.11. $500 \mathrm{nM}$ and $50 \mu \mathrm{M}$ of methamphetamine have been incubated after the stability study. In Figure 5.19, the two versions of the aptamer have been tested with the 5 and $10 \mu \mathrm{M}$ aptamer concentration incubated.

As observed in Figure 5.19A for the 75mer aptamer S-S protocol, a Rct decrease occurs at 500 $\mathrm{nM}$ and $50 \mu \mathrm{M}$. Even if the error deviation is important for the buffer and the target experiments, the trend of decrease is always observed for repetitive measurements. The $50 \mu \mathrm{M}$ methamphetamine provides an obvious decrease for both aptamer concentrations: for $5 \mu \mathrm{M}$ aptamer concentration, from $71,886 \pm 2236 \mathrm{Ohms}$ to $66,532 \pm 1988 \mathrm{Ohms}$ and for $10 \mu \mathrm{M}$ aptamer concentration, from 48,235 $\pm 2,007 \mathrm{Ohms}$ to $44,556 \pm 1,204 \mathrm{Ohms}$.

The decrease of Rct upon methamphetamine addition hypotheses are the following: (1) cationic methamphetamine molecule binds to the negative phosphate backbone, hence, improve the 
electron transfer flow by suppressing the overall negatively charge at the electrode surface (Chapter 3) (2) upon binding, a structural change of the loop (Chapter 3) enhances the electron transfer from the electrolyte to the electrode surface. These assumptions will be confirmed or rejected in Chapter 6.

In Figure 5.19B, the decrease is not as obvious as for the $75 \mathrm{mer} S-\mathrm{S}$ protocol. Nevertheless, for the 28 mer $10 \mu \mathrm{M}$, a decrease occurs at $50 \mu \mathrm{M}$ methamphetamine. The Rct value decreases from $102,490 \pm 5,123$ Ohms to $96,863 \pm 3,697$ Ohms. However, no decrease is observed for $500 \mathrm{nM}$ methamphetamine addition. We can suppose that by truncated the aptamer, fewer nucleotides are present and so, fewer interactions between the cationic methamphetamine and the negative phosphate groups of the DNA strand arise. Therefore, the Rct decrease is less consequent. The binding hypotheses will be developed in Chapter 6. An additional experiment will be completed in the next paragraph to understand the efficiency of the aptamer fixation for the disulfide protocol used.
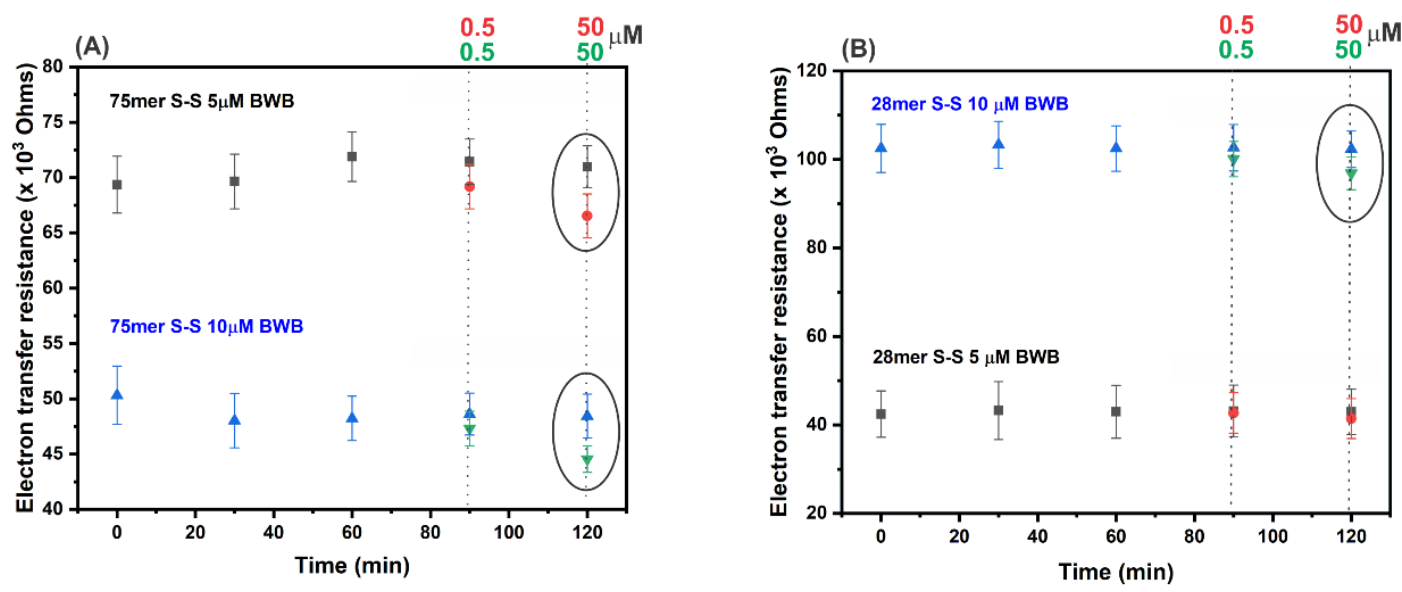

Figure 5.19. Investigation of the target incubation at $500 \mathrm{nM}$ and $50 \mu \mathrm{M}$ methamphetamine. (A) represents the 75 mer disulfide aptamer at $5 \mu \mathrm{M}$ and $10 \mu \mathrm{M}$ incubated. (B) represents the 28 mer disulfide aptamer at $5 \mu \mathrm{M}$ and $10 \mu \mathrm{M}$ incubated. 


\section{Two aptamer strands are fixed simultaneously which produces an efficient aptamer fixation}

With the use of $20 \%$ DMSO to form disulfide dimer aptamers, the enhancement of the aptamer density and the electron transfer resistance is observed. Furthermore, a significant correlation between the Rct and the aptamer density, and therefore, a better control over the formed SAM, has been proved. Finally, the methamphetamine addition to the system involves a change of the Rct.

Nevertheless, a lack of knowledge about the mechanism of fixation and the SAM conformation on the gold electrode surface with the S-S protocol appears. The next section will examine the disulfide group as well as the dimer impact on the fixation and the SAM formation. To understand if the efficiency in the aptamer fixation is related to the disulfide group or to the dimer aptamer, some experiments were done with a disulfide molecule composed on one side of a 28mer aptamer and the other side of a MCH molecule (Figure 5.20).

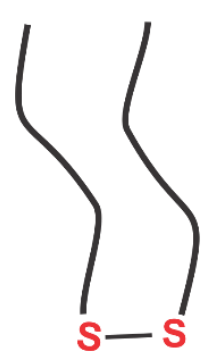

Disulfide 28mer aptamer

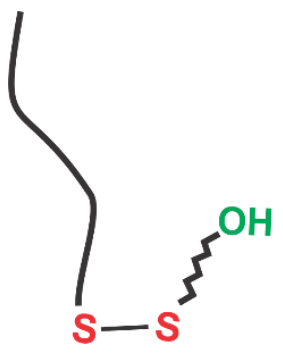

Disulfide 28mer - $\mathrm{MCH}$

Figure 5.20. Schematic representations of the disulfide 28 mer aptamer and the disulfide $28 \mathrm{mer}-$ $\mathrm{MCH}$.

As shown in Figure 5.21A, the electron transfer resistance is higher in comparison with the 20 $\%$ DMSO protocol tried in the previous section. One explanation could be that here $100 \%$ of disulfide molecule is present in solution in comparison to the $20 \%$ DMSO protocol where the conversion after $5 \mathrm{~h}$ is only $68 \%$ of disulfide molecule in solution. As a result, the efficiency of fixation is more important with the disulfide $28 \mathrm{mer}-\mathrm{MCH}$.

$\mathrm{MCH}$ molecule is fixed simultaneously with the 28 mer aptamer for the disulfide $28 \mathrm{mer}-\mathrm{MCH}$ experiment resulting in a lower aptamer density in comparison to the 28 mer $20 \%$ DMSO protocol. For example, for the $10 \mu \mathrm{M} 28$ mer aptamer concentration incubated with the $20 \%$ 
DMSO protocol, an aptamer density value of $11.9 \times 10^{12}$ molecules $/ \mathrm{cm}^{2}$ was found. By contrast, a value of $5.5 \times 10^{12}$ molecules $/ \mathrm{cm}^{2}$ was found for the disulfide $28 \mathrm{mer}-\mathrm{MCH}$ molecule.
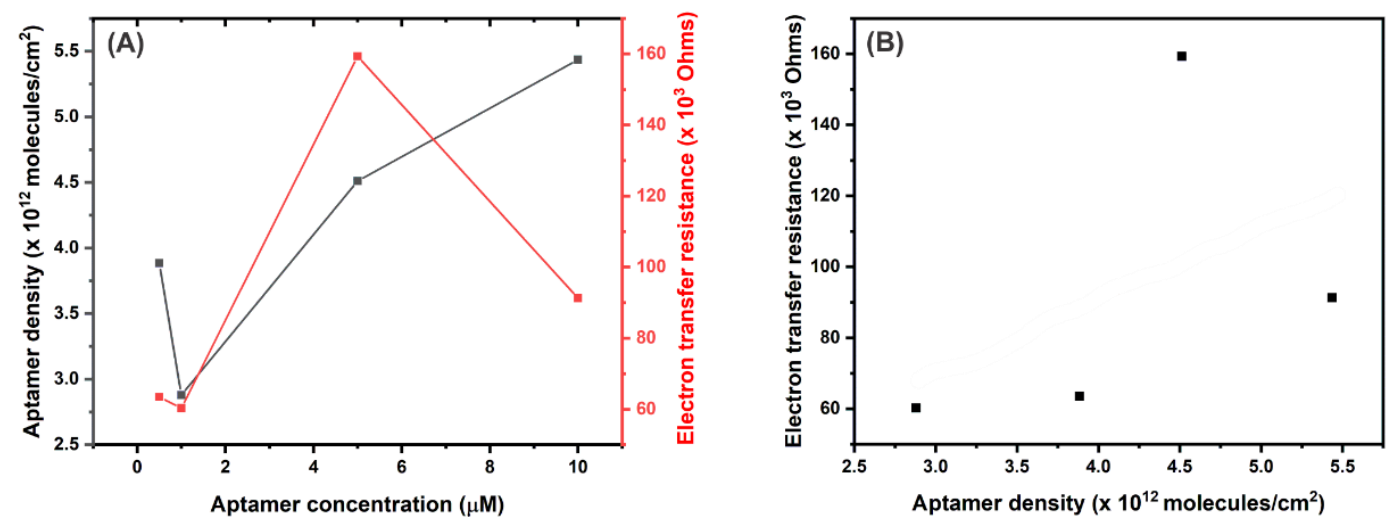

Figure 5.21. Investigation of the aptamer density and the electron transfer resistance for the disulfide $\mathrm{MCH}-28 \mathrm{mer}$ aptamer incubated concentrations.

The phenomenon of Rct decrease and aptamer density increase at $10 \mu \mathrm{M}$ concentration of aptamer incubated, similar to the two-step thiolated protocol, is observed in Figure 5.21A. This could be interpreted as a close behavior of fixation to the two-step thiolated protocol and, hence, proving that the effect shown with the $20 \%$ DMSO protocol is due to the dimer impact and not related to the specific chemistry between the gold and the disulfide group. In addition, as observed in Figure 5.21B, no correlation between the aptamer density and the Rct is shown, similarly observed in Figure $\mathbf{5 . 1 0}$ for the thiolated protocol.

With the observations done, we can suppose that the efficiency of fixation of the $20 \%$ DMSO protocol is related to the dimer formed that provides two aptamer strands fixed on the gold electrode surface at the same time and consequently, enhance the aptamer density. The disulfide chemical group could be involved in a more efficient fixation, however, it is negligible in comparison to the dimer impact. This finding is in line with the work done by Rouhana et al. ${ }^{221}$ that shows that the kinetics of fixation between the thiol and the disulfide group is similar. 


\subsection{Chapter conclusion}

Investigations on SAM have been achieved to improve the stability of the layer, and to amplify a potential detection signal by increasing the bio-recognition element density on the gold electrode surface. The co-incubation protocol shows a great response towards the stability of the SAM in the buffer solution. Nevertheless, the stability increase is mainly due to the decrease of the aptamer density on the electrode surface. More $\mathrm{MCH}$ molecules are fixed on the electrode in comparison with the two-step protocol and, therefore, the SAM created is closer to a pure MCH monolayer. The formed SAM is a highly ordered SAM. A detection signal is not visible due to the low aptamer density on the gold electrode and the weak bio-recognition event revealed in Chapter 3.

The two-step protocol provides a large signal drift related to the buffer incubation for $1 \mu \mathrm{M}$ of thiolated aptamer incubated. Some experiments, consisting of increasing the concentration of the thiolated aptamer incubated, were done. It has been proved that the initial concentration of thiolated aptamer incubated has a great impact on the stability of the layer. The signal drift related to the buffer incubation is indeed suppressed with the incubation of 5 and $10 \mu \mathrm{M}$ thiolated aptamer. However, a bad control over the aptamer density is observed with the twostep thiolated protocol. No signal change related to target incubation is generated.

A protocol that combines the stability of the SAM and sufficient aptamer density fixed was discovered. The use of dimer disulfide aptamer instead of single thiolated aptamer was done. The efficiency of fixation for the 75 and 28 mer aptamer has been proved and showed a great correlation between the electron transfer resistance and the aptamer density. This effect has not been seen with the thiolated aptamer. Moreover, we found that the DNA strand length had a great impact on the aptamer density. The shorter aptamer enables a higher aptamer density. A signal change related to target incubation has been shown for the disulfide protocol and will be further investigated in Chapter 6. 


\subsection{Experimental section}

\section{Materials and agents}

The methamphetamine aptamer sequence has been developed by AuramerBio, Wellington, New Zealand and is protected by intellectual property. It was synthesized with a thiol modification at the 5'end and purchased from AlphaDNA, Montreal, Canada. All solutions were prepared with deionized water $(18 \mathrm{M} \Omega / \mathrm{cm}$ resistivity) from a Millipore MilliQ system. Electrodes cleaning solutions used were the following: $0.1 \mathrm{M} \mathrm{NaOH}$ and $0.5 \mathrm{M} \mathrm{H}_{2} \mathrm{SO}_{4}$. The cleaning protocol is detailed in the next paragraph. Aptamer stock solutions were prepared in Tris-EDTA buffer (TE, pH 8.0) and kept frozen. Reported error bars in the figures represent the standard deviation of measurements taken from three SAM experiments that were independently fabricated and tested.

Aptamer working solution (BWB $2 \mathrm{mM}$ ) was prepared with $2 \mathrm{mM}$ Tris- $\mathrm{HCl}$ buffer $\mathrm{pH} 7.5$, containing $10 \mathrm{mM} \mathrm{NaCl}, 0.5 \mathrm{mM} \mathrm{KCl}, 0.2 \mathrm{mM} \mathrm{MgCl} 2$ and $0.1 \mathrm{mM} \mathrm{CaCl}_{2}$. Assembling buffer (AB) solution was prepared with $10 \mathrm{mM} \mathrm{Na}_{2} \mathrm{HPO}_{4}, 1 \mathrm{M} \mathrm{NaCl}$ and $1 \mathrm{mM} \mathrm{MgCl} 2$ (pH 7.3). 0.1 M phosphate buffer (PBS, pH 7.0) containing $5 \mathrm{mM} \mathrm{K}_{3}\left[\mathrm{Fe}(\mathrm{CN})_{6}\right] / \mathrm{K}_{4}\left[\mathrm{Fe}(\mathrm{CN})_{6}\right](1: 1$, v/v) was chosen as the supporting electrolyte for the EIS measurements. The chronocoulometry (CC) experiments were completed in $10 \mathrm{mM}$ Tris- $\mathrm{HCl}$ buffer (pH7.4) and a solution of $10 \mathrm{mM}$ $\mathrm{Ru}\left(\mathrm{NH}_{3}\right)_{6} \mathrm{Cl}_{3}$ in water was used.

Thiol oxidation experiments are achieved by using Ellman's reagent. 5,5'-dithiobis-(2nitrobenzoic acid) (DTNB) is used and acetyl cysteine is used as the reference thiol molecule for oxidation experiments. Prior to any aptamer handling, stock aptamer was put at room temperature for $30 \mathrm{~min}$, then was spun down for $1 \mathrm{~min}$ at $9000 \mathrm{rpm}$ and was stirred for $30 \mathrm{~s}$. A denaturation step was done by heating the aptamer solution at $95^{\circ} \mathrm{C}$ for $5 \mathrm{~min}$ on a thermoblock then cooling down with an ice bath for $10 \mathrm{~min}$. It was spun down for $1 \mathrm{~min}$ at $9000 \mathrm{rpm}$ and stir for $30 \mathrm{~s}$ at the end. Meth stock solution is kept in the freeze in milliQ water at $-20{ }^{\circ} \mathrm{C}$. Prior to any Meth molecule handling procedure, stock solution was put at room temperature for $30 \mathrm{~min}$, then was spun down for $1 \mathrm{~min}$ at $9000 \mathrm{rpm}$ and was stirred for $30 \mathrm{~s}$. Finally, the Meth solution has been prepared in the adequate BWB solution. 


\section{Preparation of planar gold electrode}

First, an electrochemical cleaning in a $0.1 \mathrm{M} \mathrm{NaOH}$ solution is done to remove previous thiol bonds. Cyclic voltammetry parameters are the following: 200 cycles, scan rate $1.0 \mathrm{~V} / \mathrm{s}$, from 0.0 to $-1.3 \mathrm{~V}$, E step $0.001 \mathrm{~V}$. Gold electrode is polished for $3 \mathrm{~min}$ with a suspension of 0.3 $\mu \mathrm{m}$ alumina followed by another polishing step for $3 \mathrm{~min}$ with a suspension of $0.05 \mu \mathrm{m}$ alumina. Then the polished electrode is sonicated for $10 \mathrm{~min}$ in ethanol and for $10 \mathrm{~min}$ in milliQ water to remove any remaining polishing agent. A second electrochemical cleaning in a $0.5 \mathrm{M} \mathrm{H}_{2} \mathrm{SO}_{4}$ solution is used. Cyclic voltammetry parameters are the following: 10 cycles, scan rate $0.1 \mathrm{~V} / \mathrm{s}$, from -0.2 to $1.6 \mathrm{~V}$, E step $0.001 \mathrm{~V}$. Cyclic voltammetry is repeated until 10 scans are stackable between each other. Finally, the cleaning of the gold electrode is checked by using cyclic voltammetry and electrochemical impedance spectroscopy (Chapter 2).

\section{Immobilization of aptamer via the two-step protocol- separated incubation of different thiolated aptamer concentrations and $1 \mathrm{mM} \mathrm{MCH}$}

All thiol aptamer solutions are prepared in assembling buffer. A preparation of $100 \mu \mathrm{L}$ is done for every concentration used. For example, the preparation of $1 \mu \mathrm{M}$ thiol aptamer solution is achieved as following. $1 \mu \mathrm{L}$ of the aptamer stock $(100 \mu \mathrm{M})$ is mixed with $99 \mu \mathrm{L}$ of assembling buffer then the mixture is heated at $95^{\circ} \mathrm{C}$ for $5 \mathrm{~min}$ and cooled down in an ice bath for $10 \mathrm{~min}$. The solution is spun down for $1 \mathrm{~min}$ at $9000 \mathrm{rpm}$ and stirred for $30 \mathrm{~s}$. The solution needs to reequilibrate at room temperature for $20 \mathrm{~min}$ at least. A $60 \mu \mathrm{L}$ of thiol aptamer solution prepared is incubated on a clean gold electrode for at least $16 \mathrm{~h}$. After $16 \mathrm{~h}$, the modified electrode is washed $3 \mathrm{x}$ with the assembling buffer solution. Then $60 \mu \mathrm{L}$ of $1 \mathrm{mM} \mathrm{MCH}$ in $\mathrm{AB}$ is incubated for $1 \mathrm{~h}$ on the modified gold electrodes. Finally, the modified electrode is washed $5 \mathrm{x}$ with the $\mathrm{AB}$ solution then it is placed in the working solution. After $5 \mathrm{~min}$ in the working solution used, the first EIS measurement can be done. EIS parameters are the following: $E_{D C} 0.23 \mathrm{~V}, E_{A C}$ $0.005 \mathrm{~V}$, tequilibration $4 \mathrm{~s}$, number of frequencies 41 , maximum frequency $10 \mathrm{kHz}$, minimum frequency $1.0 \mathrm{~Hz}$.

\section{Aptamer density determination via chronocoulometry experiment}

Chronocoulometry experiment must be done at the end of experiments (method that disrupts the SAM formed). Fast amperometry analysis is used for chronocoulometry experiments. The 
parameters used are the following: $t_{e q} 10 \mathrm{~s}, E_{e q} 0.2 \mathrm{~V}, E_{D C}-0.5 \mathrm{~V}, t_{\text {run }} 0.25 \mathrm{~s}$ and $t_{\text {interval }} 0.01 \mathrm{~s}$. A $3 \mathrm{~mL}$ of $10 \mathrm{mM}$ Tris- $\mathrm{HCl}$ is placed in a conical glass cell. A three electrodes system is used: $\mathrm{Ag} / \mathrm{AgCl}$ as the reference electrode, $\mathrm{Pt}$ wire as the counter electrode and the modified gold $(\mathrm{Au})$ as the working electrode. The $3 \mathrm{~mL}$ solution is degassed with $\mathrm{N}_{2}$ for $5 \mathrm{~min}$. The first measurement is done with the parameters written above. $Q d l$ is found. Then $15 \mu \mathrm{L}$ of $\mathrm{Ru}\left(\mathrm{NH}_{3}\right)_{6} \mathrm{Cl}_{3}$ is added to the $3 \mathrm{~mL}$ solution, the solution is degassed for 5 min with $\mathrm{N}_{2}$, the modified gold electrode is incubated in the $3 \mathrm{~mL}$ solution at the same time. The second measurement is taken after 5 min of degassing. Qtotal is found.

\section{Immobilization of aptamer via the co-incubation protocol- co-incubation of $1 \mu \mathrm{M}$ thiolated aptamer and different MCH concentrations}

All thiol aptamer/MCH solutions are prepared in assembling buffer. A preparation of $100 \mu \mathrm{L}$ is done for every ratio used. For example, the preparation of $1 \mu \mathrm{M}$ thiol aptamer/20 $\mu \mathrm{M} \mathrm{MCH}$ solution is achieved as follows. $1 \mu \mathrm{L}$ of the aptamer stock $(100 \mu \mathrm{M})$ is mixed with $2 \mu \mathrm{L}$ of 1 $\mathrm{mM}$ MCH solution, then $97 \mu \mathrm{L}$ of assembling buffer is added. The mixture is heated at $95{ }^{\circ} \mathrm{C}$ for $5 \mathrm{~min}$ and cooled down in an ice bath for $10 \mathrm{~min}$. The solution is spun down for $1 \mathrm{~min}$ at $9000 \mathrm{rpm}$ and stirred for $30 \mathrm{~s}$. The solution needs to re-equilibrate at room temperature for 20 min at least. A $60 \mu \mathrm{L}$ of thiol aptamer/MCH solution prepared is incubated on a clean gold electrode for at least $16 \mathrm{~h}$. After $16 \mathrm{~h}$, the modified electrode is washed $3 \mathrm{x}$ with the assembling buffer solution. Then $60 \mu \mathrm{L}$ of $1 \mathrm{mM} \mathrm{MCH}$ in $\mathrm{AB}$ is incubated for $1 \mathrm{~h}$ on the modified gold electrodes. Finally, the modified electrode is washed $5 \mathrm{x}$ with the $\mathrm{AB}$ solution then it is placed in the working solution. After $5 \mathrm{~min}$ in the working solution used, the first EIS measurement can be done. EIS parameters are the following: $E_{D C} 0.23 \mathrm{~V}, E_{A C} 0.005 \mathrm{~V}$, tequilibration $4 \mathrm{~s}$, number of frequencies 41 , maximum frequency $10 \mathrm{kHz}$, minimum frequency $1.0 \mathrm{~Hz}$. After each EIS measurements, the electrode is removed from the electrolyte solution, it is washed and incubated with the working solution used.

\section{Cysteine oxidation experiment for Ellman's experiment}

A $2 \mathrm{mM}$ DTNB solution is prepared in $50 \mathrm{mM}$ sodium acetate solution. Nine different acetyl cysteine solutions are prepared in milliQ water: $0.2 \mathrm{mM}, 1 \mathrm{mM}, 2 \mathrm{mM}, 5 \mathrm{mM}, 10 \mathrm{mM}, 20 \mathrm{mM}$, $50 \mathrm{mM}, 100 \mathrm{mM}$ and $200 \mathrm{mM}$. Samples for measurement are prepared as followed: $845 \mu \mathrm{L}$ of 
milliQ water $+50 \mu \mathrm{L}$ of $2 \mathrm{mM}$ DTNB solution $+100 \mu \mathrm{L}$ of $1 \mathrm{M}$ Tris- $\mathrm{HCl} \mathrm{pH} 8.0$ solution +5 $\mu \mathrm{L}$ of acetyl cysteine concentration wanted. A calibration curve is achieved by measuring the absorption at $412 \mathrm{~nm}$ of each sample using a UV-visible spectroscopy instrument. Different conditions have been tried for mild oxidation of acetyl cysteine: $\mathrm{O}_{2}$ bubbling for $10 \mathrm{~min}$, annealing + leaving at RT for a long period of time, addition of $20 \%$ DMSO. A standard solution of $1 \mathrm{M}$ acetyl cysteine is used to reach $500 \mu \mathrm{M}$ acetyl cysteine final concentration in solution. First the oxidation experiment is done then the Ellman's reagent is added to the solution.

\section{Immobilization of aptamer via the two-step protocol- separated incubation of different disulfide aptamer concentrations and $1 \mathrm{mM} \mathrm{MCH}$}

All disulfide aptamer solutions are prepared in assembling buffer. A preparation of $100 \mu \mathrm{L}$ is done for every concentration used. For example, the preparation of $1 \mu \mathrm{M}$ disulfide aptamer solution is achieved as following. $1 \mu \mathrm{L}$ of the aptamer stock $(100 \mu \mathrm{M})$ is mixed with $79 \mu \mathrm{L}$ of assembling buffer then the mixture is heated at $95{ }^{\circ} \mathrm{C}$ for $5 \mathrm{~min}$ and cooled down in an ice bath for $10 \mathrm{~min}$. The solution is spun down for $1 \mathrm{~min}$ at $9000 \mathrm{rpm}$ and stirred for $30 \mathrm{~s}$. The solution needs to re-equilibrate at room temperature for $20 \mathrm{~min}$ at least. Then $20 \mu \mathrm{L}$ of DMSO is added to the solution. The mixture is stirred thoroughly and left at RT for 5 h. $60 \mu \mathrm{L}$ of the disulfide aptamer solution is incubated on clean gold electrode for at least $16 \mathrm{~h}$. After $16 \mathrm{~h}$, the modified electrode is washed $3 \mathrm{x}$ with the assembling buffer solution. Then $60 \mu \mathrm{L}$ of $1 \mathrm{mM} \mathrm{MCH}$ in $\mathrm{AB}$ is incubated for $1 \mathrm{~h}$ on the modified gold electrodes. Finally, the modified electrode is washed $5 \mathrm{x}$ with the $\mathrm{AB}$ solution then it is placed in the working solution. After $5 \mathrm{~min}$ in the working solution used, the first EIS measurement can be done. EIS parameters are the following: $E_{D C}$ $0.23 \mathrm{~V}, E_{A C} 0.005 \mathrm{~V}, t_{\text {equilibration }} 4 \mathrm{~s}$, number of frequencies 41 , maximum frequency $10 \mathrm{kHz}$, minimum frequency. 


\section{CHAPTER 6}

\section{Investigating the range of response of the SAM upon methamphetamine addition}

\subsection{Abstract}

Taking advantage of their sensitivity, low-cost, and portability, numerous electrochemical (echem) aptasensors for a wide range of molecule detection have been reported in the last decade. However, all e-chem aptasensors are restrained to laboratory works - no commercial e-chem aptasensors are available. This reflects significant challenges of reproducibility and reliability, which are largely due to a lack of understanding of signal transduction mechanisms.

In the presence of target molecules, a transduced electrochemical signal is expected from an echem aptasensor system. Currently, to verify the specificity of the aptamer, an interferent molecule is introduced as a control. To confirm if the transduced signal is a true positive, a control using a nonspecific DNA strand instead of the specific aptamer is also required. Here, we investigated, by combining electrochemical impedance spectroscopy (EIS) and chronocoulometry (CC) methods, the response of bare gold electrode to the response of specific and nonspecific formed SAM, upon addition of methamphetamine and interferent molecules.

We found that methamphetamine and interferent molecules adsorb differently on the gold surface resulting in different e-chem signals monitored on the specific SAM. We also discovered that the difference in the nucleotides sequence and secondary structures between the aptamer and a nonspecific aptamer generates non-comparable SAMs, preventing a 
consistent comparison of the transduced signals. These experiments call for a careful evaluation of the target adsorption and the use of nonspecific DNA sequences on the signals measured for the validation of e-chem aptasensors. 


\subsection{Introduction}

Electrochemical sensors offer sensitivity, selectivity, and low cost for a wide range of target detection. The high sensitivity is a result of nanoscale interactions between the target, the recognition layer, and the solid electrode surface. Because of the high sensitivity, much information can be extracted. ${ }^{69}$ However, for e-chem aptasensors, in order to recognize a true positive binding event signal, some control experiments must be done.

First, an interaction between the target molecule and the electrode surface (target-electrode) may occur resulting in interference signals. Gold is often used as an electrode material because it is considered inert: it doesn't react with atmospheric oxygen. ${ }^{82}$ Except for the formation of a strong bond between sulfur and gold atoms, not a lot of other strong chemisorption interaction exists for the gold. Physisorption, however, is a common and well-studied phenomenon occurring between gold and a wide range of molecules and gold surfaces. ${ }^{59,61,251-256}$ Indeed, the gold surface is hydrophilic and has some affinity with nitrogen atoms. ${ }^{59,} 237$ Further, recent studies have demonstrated the adsorption of chemical compounds on gold nanoparticles (AuNPs) resulting in AuNPs aggregation. Liu and coworkers studied the adsorption of arsenite As(III) with AuNPs in a colorimetric AuNPs aptamer used in an As(III) biosensor assay. They showed that As(III) adsorption on AuNPs is strong enough to alter the DNA adsorption on AuNPs, demonstrating that observations based on the color change of AuNPs for the detection of As(III) must be interpreted carefully. The aggregation is mainly due to As(III) adsorption, not to the specific interaction between the aptamer and As(III). ${ }^{61,257}$

Electrochemical impedance spectroscopy (EIS) was also used to study the adsorption of a wide range of molecules on the gold surface. Kerner et al. ${ }^{254}$ studied the adsorption of anions on gold electrodes, obtaining adsorption rates for $\mathrm{Cl}^{-}, \mathrm{Br}^{-}, \mathrm{I}^{-}$and $\mathrm{SO}_{4}{ }^{2-} \cdot \mathrm{Br}^{-}$and $\mathrm{I}^{-}$show significant adsorption on the gold electrode surface, in contrast to $\mathrm{Cl}^{-}$and $\mathrm{SO}_{4}{ }^{2-}$.

Secondly, interactions between the DNA strand and the gold electrode (DNA-electrode) surface can result in a hypothetic transduced signal. The physisorption of DNA strands on gold surfaces is a common phenomenon that must be considered for the development of aptasensors based on gold. ${ }^{59}$ As described in Liu et al., DNA strand adsorption on the gold surface is dependent on the nucleotide sequence. ${ }^{59}$ Some nucleotide bases have a greater affinity towards gold than others. Generally, to prevent nonspecific adsorption of DNA strands or nonspecific 
adsorption of interferent/target molecules with the gold surface, blocking agents are used. These agents could be large protein molecules such as albumin or globulin, or short thiol alkyl molecules such as 6-mercapto-1-hexanol (MCH) or 1,6-hexanedithiol (HDT). ${ }^{88}$ Large protein molecules interact with the gold surface via adsorption and the short thiol alkyl molecules interact via strong bonding between the thiol group and gold atom. It creates a repelling layer to prevent any interactions with the gold surface.

Finally, the interaction between the target and the DNA (target-DNA) sequence may be nonspecific. Indeed, as described in Chapter 3, the specific interaction between methamphetamine and the aptamer is entropically driven combining hydrophobic and electrostatic interactions in a specific binding pocket. However, electrostatic interactions between other phosphate groups in the backbone of the DNA strand and the cationic methamphetamine may arise.

These three interactions (target-electrode, target-DNA, and DNA-electrode) must be understood using careful control experiments in the development of e-chem aptasensors. However, the control experiments are dependent on the e-chem measurement technique used. For example with e-chem aptasensors based on the methylene blue (MB) redox probe where MB interacts covalently or non-covalently with the aptamer, only two of the three control experiments stated above are possible. ${ }^{62}$ Indeed, the target-electrode interaction cannot be directly observed because a labelled DNA strand is required to obtain an e-chem signal.

Numerous investigations of molecule adsorption effect on AuNPs have been performed for AuNP aptasensors. However, no such investigations have been undertaken for the adsorption study of molecules on a gold electrode surface for use in e-chem aptasensor. Similarly, strong affinity between small molecules and gold electrode surface may be expected.

The use of a blocking agent for the e-chem aptasensor experiment is needed, especially for the detection of methamphetamine. Meth is a small cationic molecule that is part of the family of phenethylamine compounds. Amine groups have a strong affinity with the gold surface, and Meth is a polar molecule that can interact with the hydrophilic gold surface. ${ }^{59} \mathrm{MCH}$ is used as a blocking agent, nevertheless, fixation on the gold electrode is not comparable to a pure $\mathrm{MCH}$ layer due to perturbations caused by the DNA strands. Hence, some nonspecific sites are present on the gold electrode, as shown in Figure 6.1.

Control experiments using nonspecific DNA strands are of limited value due to the differences in fixation caused by the different DNA structures. The different nucleotide sequences will 
cause a different conformational structure on the gold surface and, will result in the creation of a different SAM to the original SAM. As a consequence, the comparison of the two systems may not be reliable.

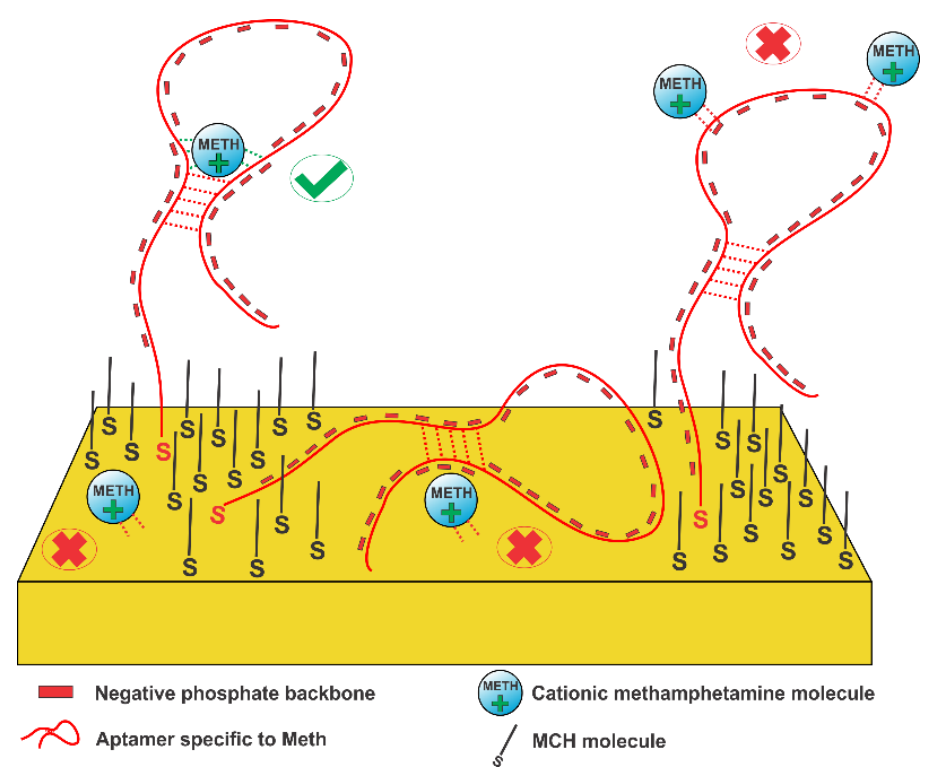

Figure 6.1. Schematic of an e-chem aptasensor system on a gold electrode surface. Possible nonspecific interactions involving induced Meth are represented.

A complete investigation of the different transduced signals that could be generated by targetelectrode, target-blocking layer, DNA-electrode and target-DNA backbone interactions, and the target and a randomized aptamer (negative aptamer) were conducted using EIS. Interferent molecules such as amphetamine and MDMA were used to have a better understanding of the meth interaction on each part of the system. Finally, chronocoulometry was used to control the aptamer density for the use of a randomized aptamer, providing consistency in the control experiment.

We found that the methamphetamine molecule has a significant affinity for the gold electrode surface. Structurally similar molecules such as amphetamine and MDMA don't adsorb on the gold surface. By using an anti-estradiol aptamer with the same number of nucleotides but a different secondary structure to the meth aptamer, we discovered that the fixation on the gold electrode surface is different due to the different initial Rct and aptamer density values, consequently, the anti-estradiol aptamer was not chosen as a control experiment. Another control was designed with a randomized sequence of the meth aptamer, without a specific secondary structure formed. The aptamer density and Rct values are comparable to the ones 
obtained with the meth aptamer. A decrease of Rct is observed with the randomized sequence, thus the control has been validated and the Rct decreases have been confirmed to be a cause of the methamphetamine adsorption on the gold surface which generates the desorption of the DNA strands from the gold surface. These findings demonstrate that careful control experiments must be designed for aptasensor systems before undertaking sensing experiments, especially in the context of small molecule detection and modest structural change aptamers. 


\subsection{Results and discussion}

The signal induced by the methamphetamine addition obtained in Chapter 5 will be investigated in the following sections. First, a whole range of Meth concentrations will be examined for the $75 \mathrm{mer}$ and $28 \mathrm{mer}$ aptamer in different ionic strength buffers.

\section{Two different trends of $R c t$ are generated from 0 to $400 \mu \mathrm{M}$ methamphetamine addition}

In Chapter 5, an electron transfer resistance decrease upon Meth addition was detected for the 75 and 28mer disulfide aptamer protocol, however, only two Meth concentrations were tested. Here, a whole range of Meth concentration is tested for the 75 mer and the 28 mer disulfide aptamer. As shown in Figure 6.2A, an important decrease of electron transfer resistance is obtained from 0 to $150 \mu \mathrm{M}$ Meth concentration. By contrast, a Rct increase occurs in the range of $150-400 \mu \mathrm{M}$ Meth concentration.
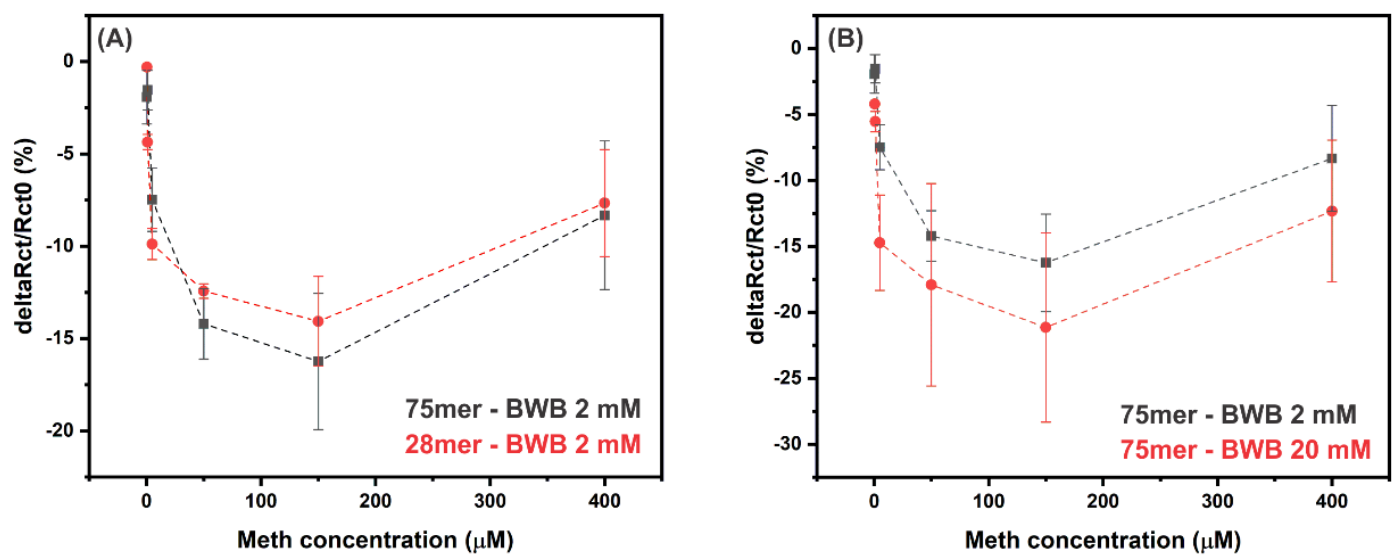

Figure 6.2. Two different experiments are represented, an overall signal induced by Meth addition occurs. (A) The 75mer and the 28mer Aptamer-2 Meth addition results and (B) the 75mer Aptamer-2 with a BWB at $2 \mathrm{mM}$ and $20 \mathrm{mM}$. 
The two trends of Rct increase and Rct decrease can be hypothesised as follows. A Rct decrease was observed for some protein detection aptasensor systems. ${ }^{107,258,259}$ Those specific proteins are positively charged macromolecules, therefore, by interacting with the negatively charged aptamers it will switch off the surface charge and provides an excess positive charge, that facilitates access of electrons from the electrolyte. A structural change of the aptamer at the electrode surface upon target addition can also explain a Rct decrease, nevertheless with the Chapter 3 findings, it is unlikely that the structural change of the aptamer could be a reason for the impedance decrease.

A Rct increase is the most common trend found in biosensing researches based on the EIS measurement technique. ${ }^{81,214,260-262}$ The structural change of the aptamer may create a more compact negative barrier at the electrode surface for the electron to cross, and hence produce an increase of the Rct. Another explanation for the Rct increase concerns the size of the target molecule. When large macromolecules such as nucleic acids or proteins are bound to the aptamer, they will repel the electron by steric hindrance, and consequently increase the Rct. In our context, none of the two reasons provided may be possible. Meth is a small molecule and no significant structural change of the aptamer is produced. Additionally, a major concern for the Rct increase observation is that it is produced at large Meth concentration incubated. This could be the result of the direct adsorption of the Meth molecule on the remaining bare gold surfaces.

In summary, the Rct decrease from $0-150 \mu \mathrm{M}$ could be the result of the electrostatic interaction between the cationic meth and the negative phosphate backbone or could be generated by a structural change of the aptamer upon the binding of the Meth. The Rct increase from $150-400 \mu \mathrm{M}$ could be the result of the direct adsorption of methamphetamine on the electrode surface or could be produced by a structural change of the aptamer upon the binding of the Meth. More experiments must be done to confirm these two statements.

\section{Truncation experiments show no significant difference in the signal detected}

The EIS results collected with the 75mer and 28 mer aptamer in Figure 6.2A are comparable. The results found are in line with the Chapter 3 findings, indeed the $28 \mathrm{mer}$ aptamer retains the binding pocket discovered. However, the value for $75 \mathrm{mer}$ is slightly more significant. The small difference in the relative electron transfer could be explained by the difference in the initial electron transfer resistance between the $28 \mathrm{mer}$ and $75 \mathrm{mer}$ formed SAMs. Indeed, 
because the initial $R c t$ for the 28 mer SAM is greater than the initial $R c t$ for the 75 mer SAM, the relative electron transfer resistance would be smaller for the 28 mer aptamer.

Here, we showed that the truncation does not affect the transduced signal. Consequently, the electrostatic interaction between the cationic Meth molecules and the negative phosphate groups stated previously as one of the major interactions occurring is disproved. Indeed, if this interaction was predominant, we would observe a smaller Rct decrease for the 28 mer aptamer. Another experiment, where we modify the ionic strength of the incubation buffer, is undertaken in the following section.

\section{lonic strength experiments reject the hypothesis of major electrostatic interactions}

In order to confirm that the transduced signal observed is not due to the electrostatic interaction between the Meth and the phosphate backbone, an experiment that consists of using two BWB with different ionic strength is done. The comparison between the 75 mer aptamer with a BWB at the ionic strength of $5.85 \mathrm{mM}$ (BWB $2 \mathrm{mM}$ ) and a BWB at the ionic strength of $58.5 \mathrm{mM}$ (BWB $20 \mathrm{mM}$ ) can provide us further insights into the electrostatic behavior of the interaction between the Meth and the aptamer.

This experiment has been designed based on Cowan et al. ${ }^{119}$ works. They modified the ionic strength of the binding buffer and monitored via isothermal titration calorimetry (ITC) the difference of the binding enthalpy produced. They were able to do a quantitative evaluation of hydrogen bonding and electrostatic interactions of the neomycin B binding with its specific RNA.

Here, the two initial $R c t$ are similar, so the comparison of the relative electron transfer resistance difference is consistent. In Figure 6.2B, a more significant decrease of $R c t$ is monitored with BWB $20 \mathrm{mM}$. If the electrostatic interactions had a major effect on the Rct decrease transduced signal, we would observe a less significant $R c t$ decrease with the BWB $20 \mathrm{mM}$. Indeed, in the case of BWB $20 \mathrm{mM}$, the salt concentration of the buffer is higher and consequently, more ions can interact and neutralize the negative phosphate backbone groups. Therefore, less cationic Meth could interact with them and generate the Rct decrease transduced signal. 
The results obtained in Figure 6.2B confirm the statement that the electrostatic interaction between the cationic meth and the negative phosphate backbone is not the major producer of the transduced signals observed from $0-400 \mu \mathrm{M}$. Here, without substantial control experiments, no interpretation of the signals observed is possible. Some control experiments will be achieved in the next sections to get a physical meaning of each trend observed.

\section{Direct adsorption of the methamphetamine on the gold electrode surface is observed}

EIS measurement technique allows us to monitor the adsorption of chemical molecules on an electrode surface. ${ }^{254,263}$ Here, the adsorption in different BWB conditions of the Meth molecule on the bare gold electrode surface and on a MCH modified gold electrode surface is tested.

As observed in Figure 6.3A, after four impedance measurements in the buffer, a gradual increase of Meth concentration each $30 \mathrm{~min}$ is achieved until reaching $400 \mu \mathrm{M}$ of Meth at 270 min. From $0.5 \mu \mathrm{M}$ to $5 \mu \mathrm{M}$ Meth concentration, no significant change in the electron transfer resistance is observed for each condition tested. At $50 \mu \mathrm{M}$ Meth concentration, an important increase in the electron transfer resistance occurs for the two bare gold experiments. No change in $R c t$ is noticed for the $\mathrm{MCH}$ layer.

At concentrations higher than $50 \mu \mathrm{M}$, different trends are observed for each experiment. The bare gold surface at BWB $20 \mathrm{mM}$ shows a continuous increase until $400 \mu \mathrm{M}$ Meth concentration. The bare gold surface at BWB $2 \mathrm{mM}$ shows a little Rct increase in comparison to the BWB $20 \mathrm{mM}$ experiment. By contrast, the $\mathrm{MCH}$ layer affords no Rct change upon Meth addition from 0.5 to $400 \mu \mathrm{M}$ for the two BWB ionic strengths.

In Figure 6.3B, the electron transfer resistance plotted in function of the Meth concentration gives the known Langmuir behavior for BWB $2 \mathrm{mM}$ and BWB $20 \mathrm{mM}$ on the bare gold experiments for the concentration range used. Therefore, a Langmuir isotherm has been drawn for each BWB used and a linearization was done in Figure 6.3C to obtain an affinity constant of the adsorption of the Meth with the gold electrode surface for each ionic strength condition in the specific range of concentration used. BWB $20 \mathrm{mM}$ is the ionic strength that shows the greatest affinity for the Meth molecule to adsorb on the gold electrode surface in the specific range of Meth concentration used. 

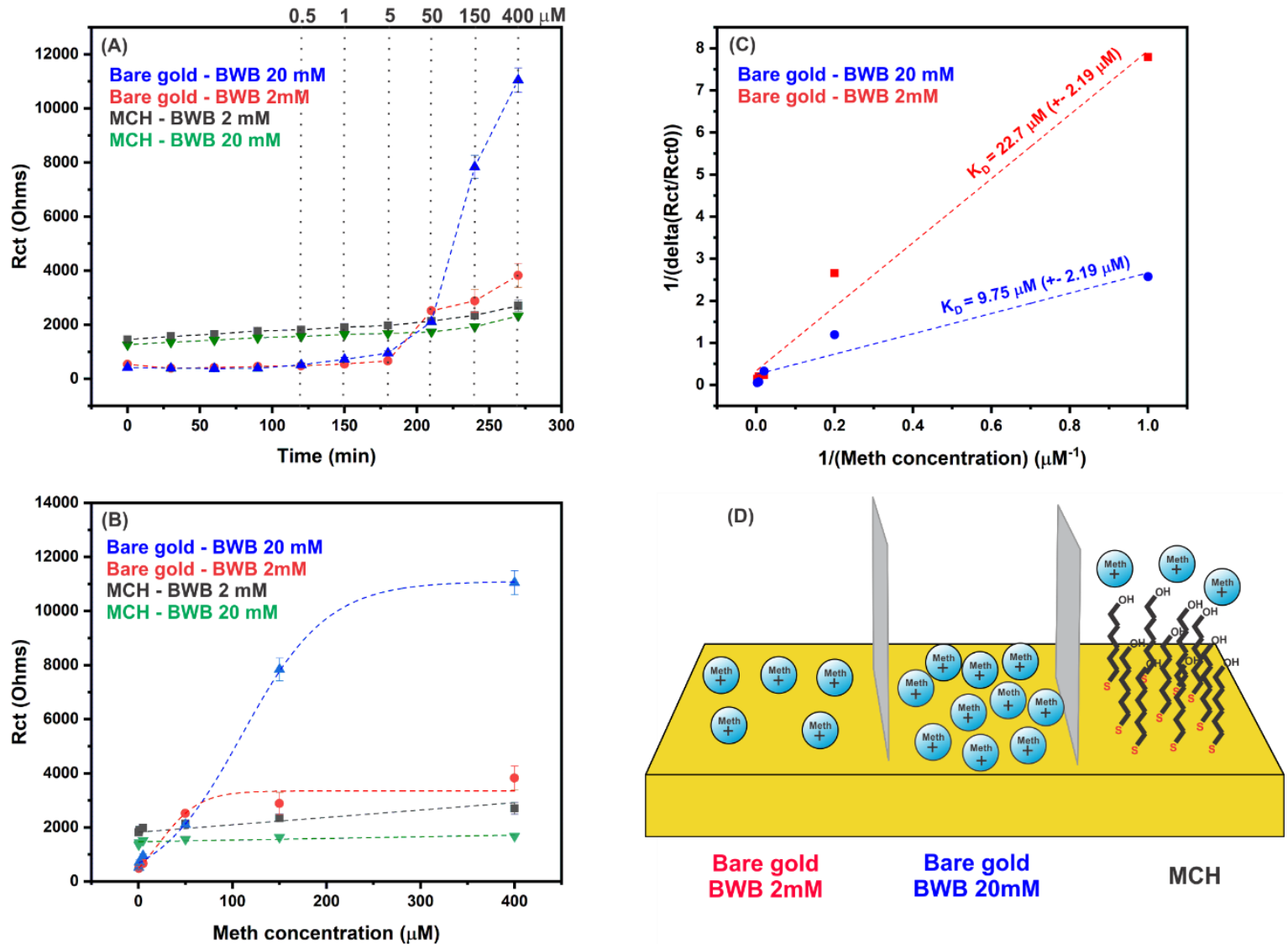

Figure 6.3. The methamphetamine adsorption in different BWB ionic strength on bare gold electrode and on $\mathrm{MCH}$ are monitored via EIS. The electron transfer resistance resulting of adsorption are plotted in function of the (A) time and (B) Meth concentration. (C) A linearization of the Langmuir isotherm, in the range of concentration used, is achieved. (D) Hypothetic illustration of the physical meaning of the adsorption results.

In Figure 6.3D, an illustration of the adsorption experiments results is represented. First, the $\mathrm{MCH}$ layer prevents the direct interaction of the methamphetamine molecule with the bare gold surface at the two different ionic strengths tested. The formed SAM is well-packed resulting in good stability of the SAM and efficient blocking agent function. ${ }^{225,258}$

The second main observation is the difference in methamphetamine adsorption for the two ionic strengths tested. Indeed, a more significant Rct increase is observed for the higher ionic strength buffer. It could be explained by the decrease of the water solubility parameter of the methamphetamine when the ionic strength is increased. ${ }^{264,265}$ Consequently, the equilibrium of the methamphetamine molecule at the interface electrode-solution is pushed towards the 
electrode surface, resulting in more methamphetamine molecules adsorb at the electrode surface. This phenomenon is well-known in biochemistry. ${ }^{264,265}$

A relationship between the significant increase of Rct related to the adsorption of the Meth molecule on the gold surface with the significant decrease of Rct observed with the functionalised electrode (Figure 6.2) could be established. $\mathrm{MCH}$ molecule is used in the sensing experiments in Figure 6.2 to avoid the nonspecific interactions between the target and the electrode surface. However, even if $\mathrm{MCH}$ is used in the protocol of the SAM formation, the surface will not behave as a pure $\mathrm{MCH}$ layer. The degree of similarity with a pure $\mathrm{MCH}$ layer is not known and thus it is not possible to make any conclusion at this point.

Concerning the Rct increase observed in the sensing experiments from 150 to $400 \mu \mathrm{M}$, an increase in Rct is produced by the adsorption of Meth on the gold surface. This would conclude that the Rct increase from 150 to $400 \mu \mathrm{M}$ is a nonspecific signal produced by the adsorption of the Meth on the gold electrode surface.

For a better fundamental understanding of the Meth interaction with the bare gold electrode surface and for being able to get more insight into the sensing experiments results obtained, structural similar compounds the amphetamine and the 3,4-Methylenedioxymethamphetamine (MDMA) molecules are used in the next section.

\section{No direct adsorption on gold surface is observed for MDMA and amphetamine}

To determine the nature of the methamphetamine adsorption on the bare gold electrode surface, the adsorption of structurally similar molecules was explored in the following section. As shown in Figure 6.4, the amphetamine and the MDMA molecules have been used.

Methamphetamine<smiles>CNC(C)Cc1ccccc1</smiles>

Polar surface area $12 \AA^{2}$ Molecular weight $149.2 \mathrm{~g} / \mathrm{mol}$ Water solubility $6.2 \mathrm{mmol} / \mathrm{L}$ pKa 10.2

\section{Amphetamine}<smiles>CC([NH3+])Cc1ccccc1</smiles>

Polar surface area $26 \AA^{2}$ Molecular weight $135.2 \mathrm{~g} / \mathrm{mol}$ Water solubility $12.9 \mathrm{mmol} / \mathrm{L}$ pKa 10.0
3,4-Methylenedioxymethamphetamine<smiles>C[NH2+]C(C)Cc1ccc2c(c1)OCO2</smiles>

Polar surface area $31 \AA^{2}$

Molecular weight $193.3 \mathrm{~g} / \mathrm{mol}$

Water solubility $16.7 \mathrm{mmol} / \mathrm{L}$ pKa 10.1

Figure 6.4. Skeletal formula of the predominant forms at $\mathrm{pH} 7.5$ of the methamphetamine, the amphetamine, and the 3,4-Methylenedioxymethamphetamine (MDMA). The polar surface area, the molecular weight, the water solubility and the pKa of each molecule are represented. 
In Figure 6.4, the three skeletons formula of Meth, amphetamine, and MDMA are represented. As shown with their $\mathrm{pKa}$, their predominant forms in solution are cationic at $\mathrm{pH}$ 7.5. The molecular weights of the three molecules are comparable. MDMA is bigger due to the methylenedioxy part attached to the methamphetamine molecule. This chemical group affords also a higher polar surface area for the MDMA molecule. Amphetamine has a comparable polarity to MDMA. However, the polarity of the methamphetamine is lower because of the Nmethyl group attached to the nitrogen atom. ${ }^{266}$ The water solubilities are different for each component: the methamphetamine has the lower and the MDMA has the greater.

One similarity between the three molecules is the cationic nitrogen. Kamalakannan et al. ${ }^{267}$ reported a computational adsorption study of hydrophobic and hydrophilic liquids on $\mathrm{Au}$ surface. As already observed in Liu et al. ${ }^{59}$, the nitrogen atom has a strong affinity with the gold surface. Moreover, the affinity is stronger when the nitrogen is cationic. ${ }^{267}$ However as observed with the results in Figure 6.5, the adsorption is different for each molecule despite the cationic nitrogen.

As observed in Figure 6.5, no Rct increase occurs for the MDMA and amphetamine molecules in the range of concentration and at the two different BWB ionic strength tested. By contrast to the Meth molecule, we can hypothesise that the MDMA and the amphetamine molecules do not have a significant adsorption on the gold surface in the concentration range of $0-400 \mu \mathrm{M}$. 

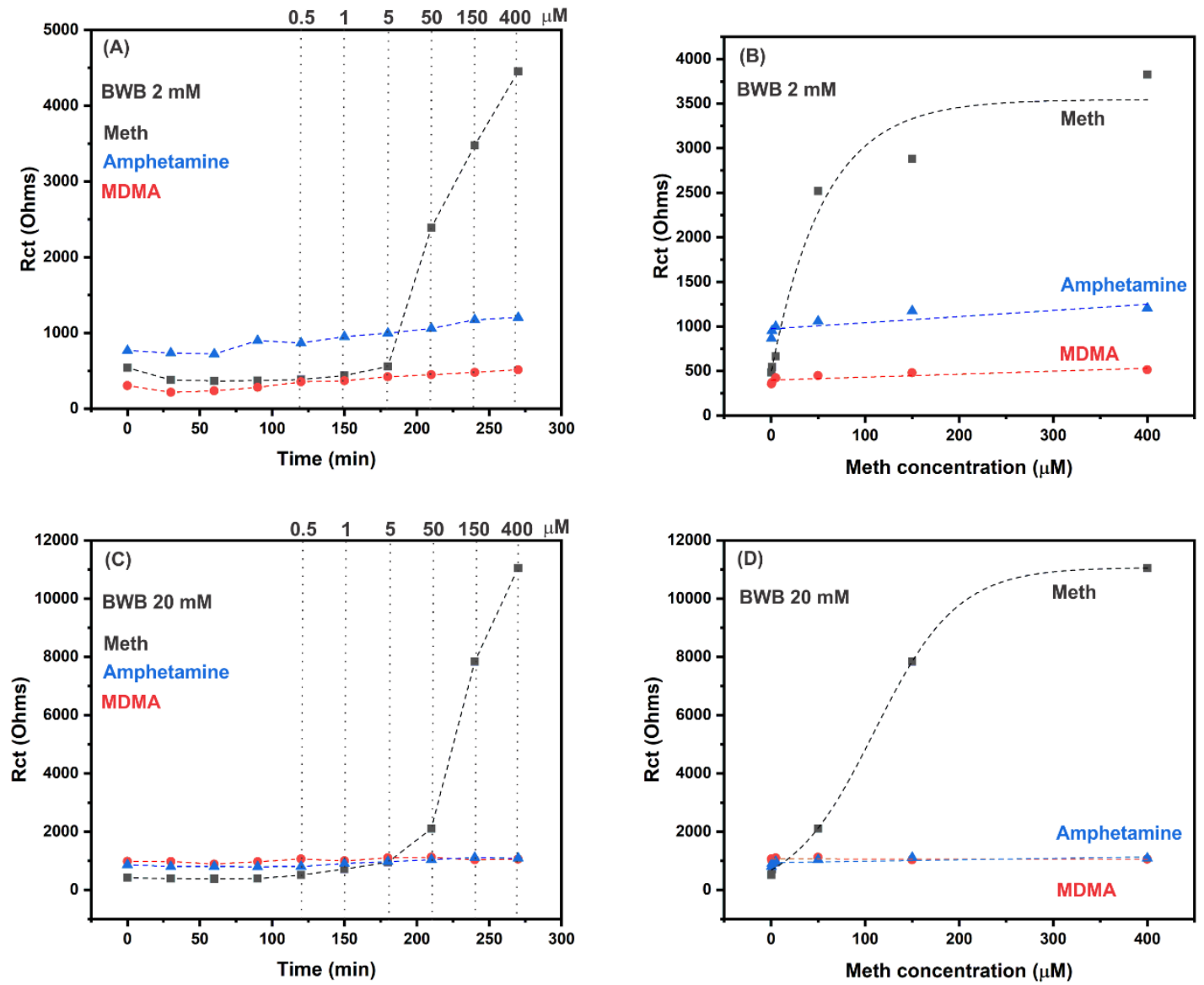

Figure 6.5. Methamphetamine, amphetamine, and MDMA adsorption in BWB $2 \mathrm{mM}$ and BWB 20 $\mathrm{mM}$ on bare gold electrode are monitored via EIS. The resulting electron transfer resistance of adsorption are plotted in function of the time (A and C) and concentration (B and D).

\section{Water solubility and polarity affect the adsorption of methamphetamine on the gold surface}

The major difference between the three molecules tested is the polarity and the water solubility. First, the gold surface is hydrophilic. ${ }^{59,237}$ Consequently, the more the molecules are polar the more the affinity with the gold surface will be great. However, here the two molecules with the greater polarity (MDMA and amphetamine) show a smaller affinity with the electrode surface. So the hydrophilic nature of the gold surface is not the explanation for the adsorption.

The water solubility is smaller for the methamphetamine so it could explain the greater adsorption of methamphetamine. Indeed, the equilibrium of the methamphetamine at the electrode-solution interface is more pushed towards the electrode surface. 
Two hypotheses of fixation could be explored: First, in the study from Kamalakannan et al. ${ }^{267}$, they showed that the benzene ring and the hydrocarbon chain interact non-covalently with the gold surface by being placed in parallel to this one. Because of the great polarity of MDMA and amphetamine, they are repelled from the electrode surface. Indeed, the positive potential applied during the measurement disrupted the adsorption of MDMA and amphetamine (Figure 6.6A). An example of the polarity impact on adsorption/absorption interaction between Meth and amphetamine is that the Meth can better penetrate the blood-brain barrier due to its low polarity in comparison with amphetamine. ${ }^{266}$

A second hypothesis could be the formation of a self-assembled monolayer on the gold surface with the methamphetamine molecule. The nitrogen group could interact with the gold electrode. Instead of being placed in parallel to the surface, the molecule will be placed perpendicular to the surface with the nitrogen group interacting with the gold surface as observed in Figure 6.6B. As the polarity is modest for the methamphetamine molecule when the positive potential is applied, the formed SAM of methamphetamine will not be disrupted. By contrast to the MDMA and amphetamine molecules where their polarity could be the reason why they are repelled from the surface when a positive potential is applied. Furthermore, the MDMA molecule will not be able to form a SAM because of the steric hindrance generated by its methylenedioxy end group.

(A)
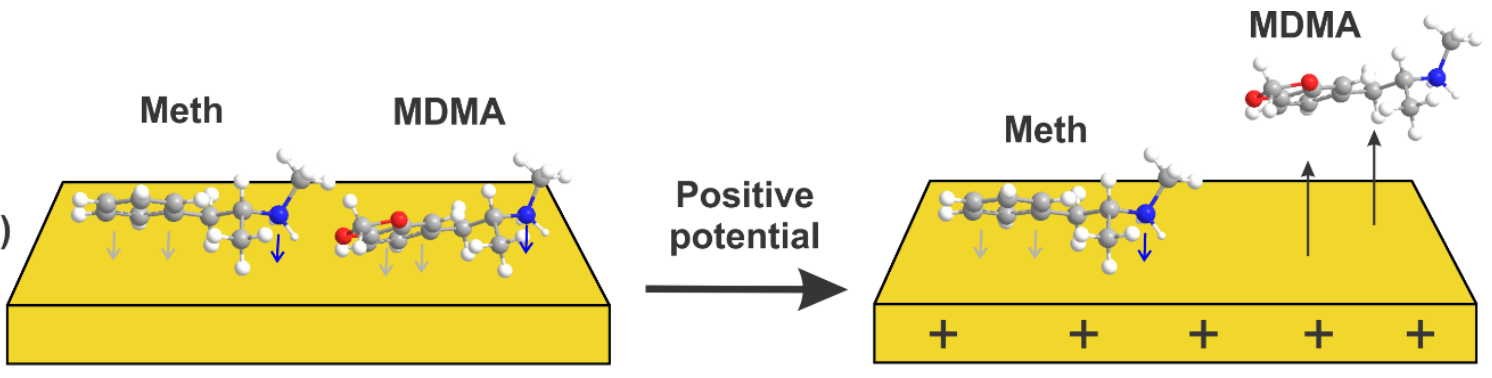

(B)
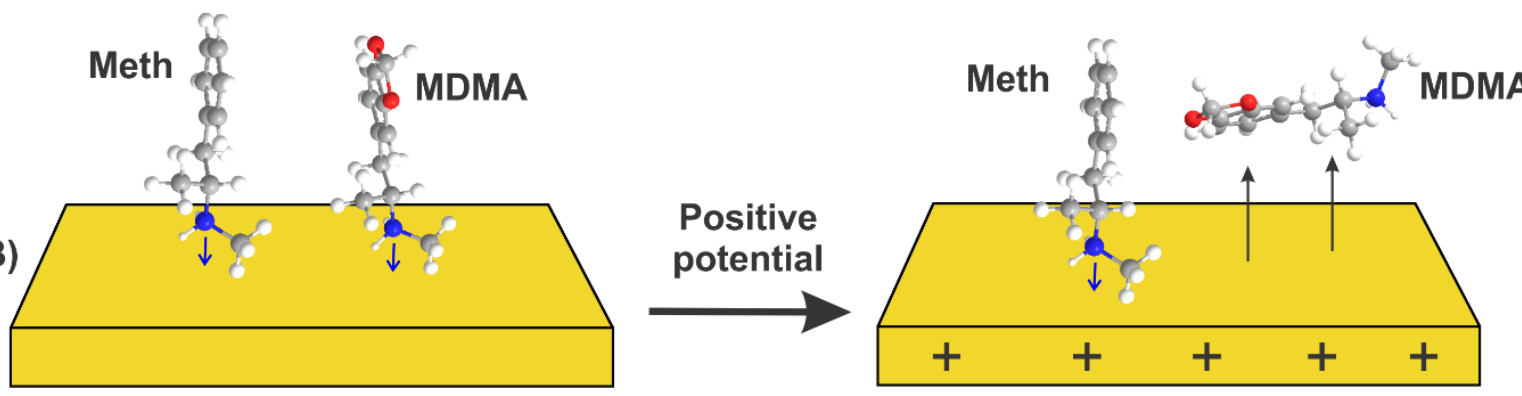

Figure 6.6. Schematic illustration of the Meth adsorption on gold surface and repulsion of MDMA from gold surface. (A) represents the lying down interaction and (B) the standing up interaction. 


\section{When a Meth aptamer functionalised electrode is exposed to MDMA, the Rct response differs from Meth}

The addition of the MDMA molecule with the Aptamer-2 75mer in BWB $2 \mathrm{mM}$ was studied and the comparison with the Aptamer-2 75mer response to the Meth addition in BWB $2 \mathrm{mM}$ is observed in Figure 6.7. No Rct decrease is observed for the addition of MDMA molecule to the Aptamer-2 75mer. With the previous adsorption experiments that show no MDMA adsorption on the gold surface in this concentration range, the relationship between the Rct decrease and the strong adsorption of Meth on the gold electrode surface is partially confirmed. It shows that without molecule adsorption on the gold electrode surface, no Rct decrease is detected for the disulfide sensing experiments designed.

An interesting observation for the aptamer functionalized electrode is the increase of $R c t$ upon MDMA addition from 50 to $400 \mu \mathrm{M}$. Indeed, it can not be attributed to the direct adsorption of MDMA on the bare gold electrode surface because no EIS adsorption signal is observed for MDMA in this range of concentration (Figure 6.5). This increase could be the effect of an interaction between the DNA strand and the MDMA molecule. To confirm the nonspecificity of the signal detected through the Rct decrease, a final control experiment by using a negative aptamer must be done.
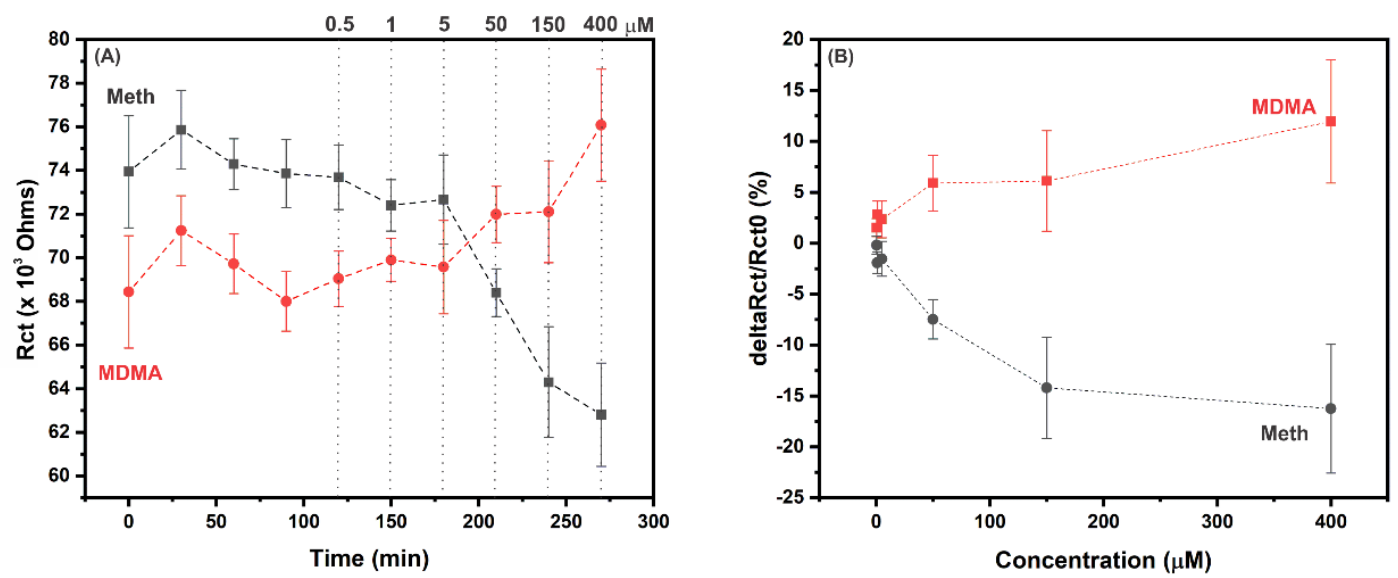

Figure 6.7. Comparison of the MDMA and the Meth addition on the disulfide Aptamer-2 system. (A) The electron transfer resistance is plotted in function of the time. (B) The relative electron transfer resistance is plotted in function of the concentration added. 


\section{Control experiments using negative aptamers introduce ambiguities}

With the previous investigations, we have enough doubt about the true positivity of the transduced signals observed in Figure 6.2. A final experiment to confirm the real nature of the transduced signals monitored must be done. This experiment consists of using another DNA strand of a similar length that is not specific towards the Meth molecule..$^{62,238}$

First, a 35 mer $17 \beta$-estradiol aptamer (E2 aptamer) has been chosen. ${ }^{60,81}$ The $17 \beta$-estradiol is a molecule structurally different from the methamphetamine so no specific interactions are expected between the methamphetamine and the E2 aptamer. A similar disulfide protocol of SAM formation is used.

In Figure 6.8, the comparison between the 28mer Meth aptamer and the 35mer E2 aptamer results upon the Meth addition is observed. No Rct decrease in the $0-150 \mu \mathrm{M}$ concentration range occurs for the E2 aptamer upon Meth addition. However, as shown in Figure 6.8 and Table 6.1, the initial $R c t$ and the aptamer density are different between the Meth aptamer experiment and the E2 aptamer experiment. With the use of EIS and CC measurement techniques, additional control experiments can be done to confirm the results obtained from the negative aptamer control experiment. As the aptamer density and the Rct are different between the Aptamer-2 - 28mer and the E2-35mer, the comparison upon Meth addition is not acceptable.
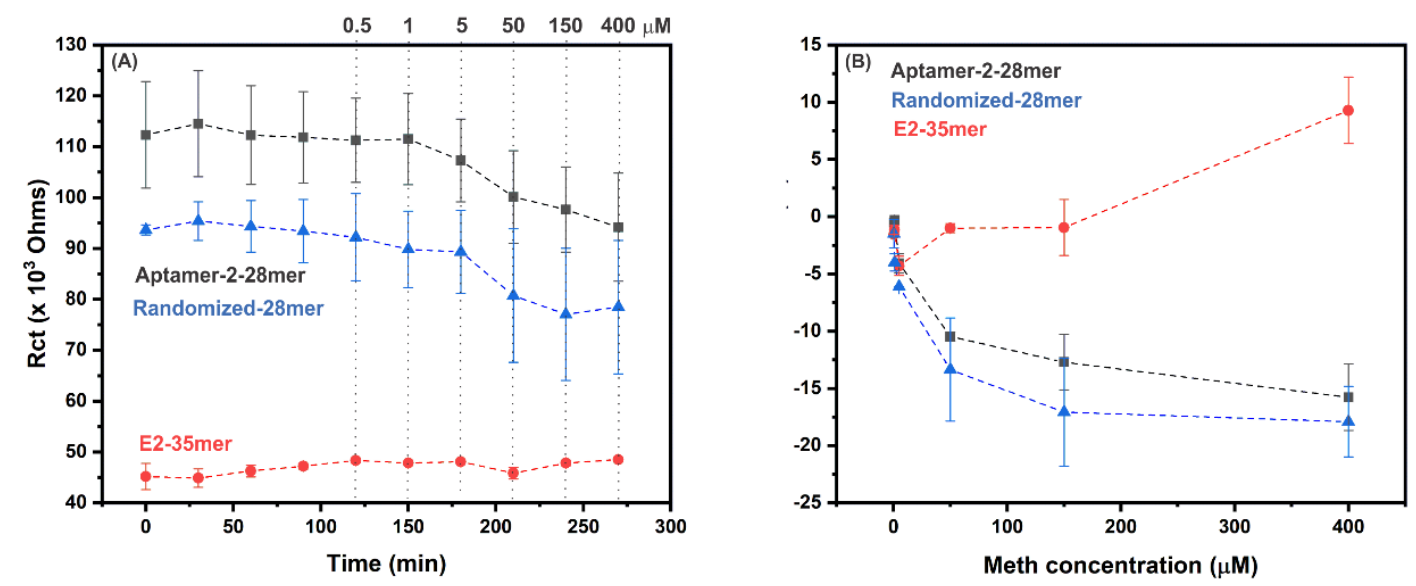

Figure 6.8. Meth sensing experiments for the Aptamer-2 - 28mer, the randomized - 28mer and the E2 -35 mer. The results are observed in the function of the time (A) and in the function of the Meth concentration (B). 
As shown in Table 6.1, the nucleotide content is not the same between the E2 35mer and the Aptamer-2 - 28mer. That's why for another control experiment, we decided to use a randomized aptamer of the Aptamer-2 - 28mer called the Randomized - 28mer.

\begin{tabular}{c|c|c|c} 
& $\begin{array}{c}\text { G content/T } \\
\text { content }\end{array}$ & $\begin{array}{c}\text { Initial } \text { Rct } \\
(\mathbf{O h m s})\end{array}$ & $\begin{array}{c}\text { Aptamer density } \\
\left(\mathbf{m o l e c u l e s} / \mathbf{c m}^{2}\right)\end{array}$ \\
\hline E2-35mer & $13 \mathrm{G} / 8 \mathrm{~T}$ & $45183 \pm 4578.1$ & $(4.3 \pm 1.4) \times 10^{12}$ \\
\hline Aptamer-2 - 28mer & $6 \mathrm{G} / 12 \mathrm{~T}$ & $112330 \pm 6078.11$ & $(9.5 \pm 3.9) \times 10^{12}$ \\
\hline Randomized - 28mer & $6 \mathrm{G} / 12 \mathrm{~T}$ & $94330 \pm 6578.1$ & $(8.7 \pm 3.7) \times 10^{12}$
\end{tabular}

Table 6.1. Nucleotides content, initial electron transfer resistance and aptamer density for the three different DNA strands used for SAM formation using the disulfide protocol.

In Figure 6.8, the results observed for the randomized - 28mer are comparable to the results obtained with the Aptamer-2 - 28mer. Moreover, as shown in table 6.1, the initial Rct and the aptamer density are worthy of comparison between the two DNA strands. As a consequence, the control experiment using the Randomized - 28mer DNA strand is acceptable. As expected, the Rct decrease appears for the Randomized - 28mer. Consequently, we have proven that the Rct decrease comes from the direct adsorption of the methamphetamine on the gold electrode. The hypotheses on the physical meaning of the Rct decrease and Rct increase will be explored in the next section.

\section{Meth adsorption on gold surface causes a displacement of DNA strands}

In Figure 6.9, we schematized two hypotheses for the $R c t$ decrease from $0-150 \mu \mathrm{M}$ and two hypotheses for the Rct increase from $150-400 \mu \mathrm{M}$. In Figure 6.9A, the DNA strands are chemisorbed to the gold surface with the sulfur group. They are in the "lying-down" conformation. By interacting with the gold surface, the methamphetamine generates a "standing-up" of the DNA strands resulting in a decrease of the electron transfer resistance. Then, as observed in Figure 6.9C, the accumulation of methamphetamine produces an increase in the electron transfer resistance.

In Figure 6.9B, the DNA strands are not chemisorbed due to the number of DNA strands on the electrode that prevents the chemisorption to occur. Upon the methamphetamine interaction 
with the gold surface, the DNA strands are disassembled from the gold surface. Then, in Figure 6.9D, the accumulation of methamphetamine produces an increase in electron transfer resistance.

\section{Rct decrease hypotheses upon Meth addition \\ (0 to $150 \mu \mathrm{M})$}
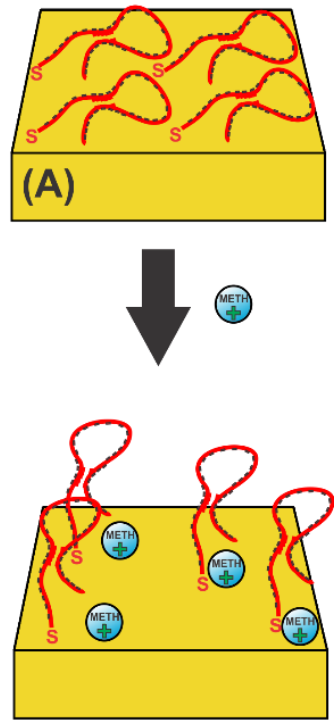

Lying down to standing up conformation because of Meth adsoprtion

Aptamer specific to Meth
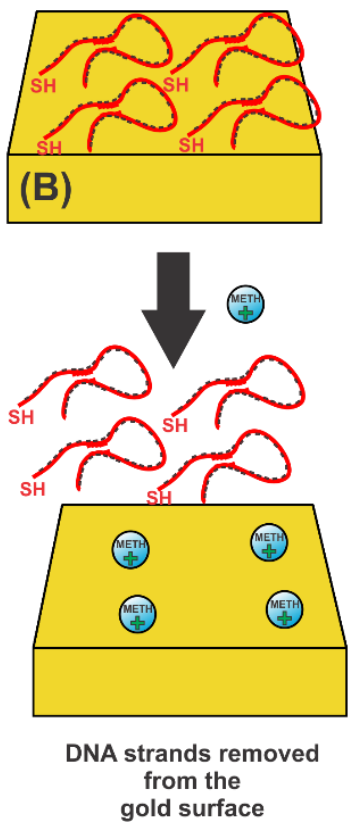
gold surface
Rct increase hypothesis upon Meth addition

$(150$ to $400 \mu \mathrm{M})$
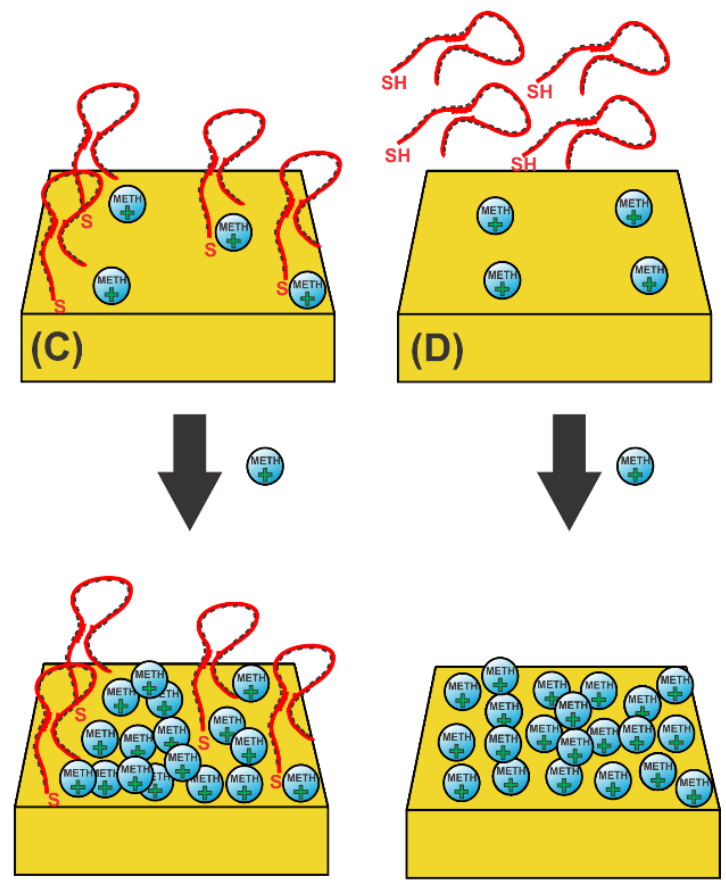

Accumulation of Meth on gold electrode surface

Cationic methamphetamine molecule

Figure 6.9. Schematic representations of the hypothetic explanation of the $R c t$ decrease and the $R c t$ increase observed during the sensing experiments in the range of $0-400 \mu \mathrm{M}$ Meth concentration. (A) and (B) represent two hypotheses for the concentration range $0-150 \mu \mathrm{M}$, and (C) and (D) two hypotheses for the concentration range $150-400 \mu \mathrm{M}$. 


\subsection{Chapter conclusion}

Here, we thoroughly investigated transduced electrochemical signals obtained with the Aptamer-2 and the disulfide protocol developed in Chapter 5 by using the electrochemical impedance spectroscopy measurement technique. We obtained two distinct EIS signal trends: a $R c t$ decrease from 0 to $150 \mu \mathrm{M}$ Meth concentration and a $R c t$ increase from $150 \mu \mathrm{M}$ Meth concentration. No explanations could be done for the trends observed without undertaking additional control experiments.

First, the direct adsorption of methamphetamine on the gold surface was done. Significant adsorption was detected at $50 \mu \mathrm{M}$ Meth concentration. We observed that the Meth adsorption is more significant in BWB with higher ionic strength. By using interferent similar molecules such as MDMA and amphetamine, an explanation about the adsorption behavior of the Meth was done. Indeed, for MDMA and amphetamine no adsorption signal is detectable due to their higher polarities that prevent the interaction of the molecule with the gold surface. $\mathrm{MCH}$ is a good blocking agent because no Meth adsorption is monitored on an $\mathrm{MCH}$ layer.

With the first control done, a hypothesis of nonspecificity concerning the Rct decrease at 50 $\mu \mathrm{M}$ and the Rct increase from $150 \mu \mathrm{M}$ for the sensing experiments emerges. The Rct decrease trend may be due to the important Meth adsorption on the gold surface. Indeed, as shown previously MDMA doesn't adsorb on the gold electrode and hence, no Rct decrease is observed upon MDMA addition on the Aptamer-2 system. The Rct increase trend may be due to a large amount of Meth deposition on the bare gold electrode surface that blocks the electron transfer.

A second control experiment was achieved by using a negative aptamer. Two different DNA strands have been used: an aptamer with the same length but specific to another molecule structurally different than Meth or a randomized DNA sequence of the Aptamer-2 - 28mer. An important difference in EIS signal was monitored. Indeed, the E2 aptamer showed no signal upon the Meth addition, excepted a Rct increase from $150 \mu \mathrm{M}$ Meth concentration. However, the initial Rct and the aptamer density are significantly different from the Aptamer-2 - 28mer values. By using the randomized - 28mer sequence, the initial Rct and the aptamer density are comparable to the Aptamer-2 - 28mer and thus, the DNA strand can be used as a negative control. The Rct decrease appears with the randomized - 28mer. That's why the nonspecificity of the Rct decrease is confirmed. This EIS signal is due to the displacement of the DNA strand for the gold electrode surface produced by the Meth adsorption on the same surface. The Rct 
increase is produced by the Meth adsorption on the SAM that blocks the electron transfer. Some AFM studies must be done to observe in depth the conformation of the SAM formed with the disulfide protocol and obtaining more information concerning the signal monitored. 


\subsection{Experimental section}

\section{Materials and agents}

The methamphetamine aptamer sequence has been developed by AuramerBio, Wellington, New Zealand and is protected by intellectual property. The randomized, Meth, and E2 aptamer sequences were synthesized with a thiol modification at the 5'end, and purchased from AlphaDNA, Montreal, Canada. All solutions were prepared with deionized water $(18 \mathrm{M} \Omega / \mathrm{cm}$ resistivity) from a Millipore MilliQ system. Electrodes cleaning solutions used were the following: $0.1 \mathrm{M} \mathrm{NaOH}$ and $0.5 \mathrm{M} \mathrm{H}_{2} \mathrm{SO}_{4}$. The protocol of cleaning is detailed in the next paragraph. Aptamer stock solutions were prepared in Tris-EDTA buffer (TE, pH 8.0) and kept frozen..

Aptamer working solution with normal ionic strength (BWB $2 \mathrm{mM}$ ) was prepared with $2 \mathrm{mM}$ Tris- $\mathrm{HCl}$ buffer $\mathrm{pH} 7.5$, containing $10 \mathrm{mM} \mathrm{NaCl}, 0.5 \mathrm{mM} \mathrm{KCl}, 0.2 \mathrm{mM} \mathrm{MgCl} 2$ and $0.1 \mathrm{mM}$ $\mathrm{CaCl}_{2}$. Aptamer working solution with higher ionic strength (BWB $20 \mathrm{mM}$ ) was prepared with $20 \mathrm{mM}$ Tris- $\mathrm{HCl}$ buffer $\mathrm{pH}$ 7.5, containing $100 \mathrm{mM} \mathrm{NaCl}, 5 \mathrm{mM} \mathrm{KCl}, 2 \mathrm{mM} \mathrm{MgCl} 2$ and 1 $\mathrm{mM} \mathrm{CaCl} 2$. Reported error bars reported in the figures represent the standard deviation of measurements taken from three experiments that were independently monitored.

Assembling buffer (AB) solution was prepared with $10 \mathrm{mM} \mathrm{Na} 2 \mathrm{HPO}_{4}, 1 \mathrm{M} \mathrm{NaCl}$ and $1 \mathrm{mM}$ $\mathrm{MgCl}_{2}$ (pH 7.3). 0.1 M phosphate buffer (PBS, $\mathrm{pH}$ 7.0) containing $5 \mathrm{mM}$ $\mathrm{K}_{3}\left[\mathrm{Fe}(\mathrm{CN})_{6}\right] / \mathrm{K}_{4}\left[\mathrm{Fe}(\mathrm{CN})_{6}\right](1: 1, \mathrm{v} / \mathrm{v})$ was chosen as the supporting electrolyte for the EIS measurements.

The chronocoulometry (CC) experiments were completed in $10 \mathrm{mM}$ Tris-HCl buffer (pH7.4) and a solution of $10 \mathrm{mM} \mathrm{Ru}\left(\mathrm{NH}_{3}\right)_{6} \mathrm{Cl}_{3}$ in water was used.

Prior to any aptamer handling, stock aptamer was put at room temperature for $30 \mathrm{~min}$, then was spun down for $1 \mathrm{~min}$ at $9000 \mathrm{rpm}$ and was stirred for $30 \mathrm{~s}$. A denaturation step was done by heating the aptamer solution at $95{ }^{\circ} \mathrm{C}$ for 5 min on a thermoblock then cooling down with an ice bath for $10 \mathrm{~min}$. It was spun down for $1 \mathrm{~min}$ at $9000 \mathrm{rpm}$ and stir for $30 \mathrm{~s}$ at the end.

Meth, amphetamine and MDMA stock solutions are kept in the freeze in milliQ water at - 20 ${ }^{\circ} \mathrm{C}$. Prior to any Meth, amphetamine or MDMA molecule handling procedures, stock solutions were put at room temperature for $30 \mathrm{~min}$, then were spun down for $1 \mathrm{~min}$ at $9000 \mathrm{rpm}$ and were 
stirred for $30 \mathrm{~s}$. Finally, the Meth, amphetamine and MDMA solutions have been prepared in the adequate BWB solution.

\section{Preparation of planar gold electrode}

First, an electrochemical cleaning in a $0.1 \mathrm{M} \mathrm{NaOH}$ solution is done to remove previous thiol bonds. Cyclic voltammetry parameters are the following: 200 cycles, scan rate $1.0 \mathrm{~V} / \mathrm{s}$, from 0.0 to $-1.3 \mathrm{~V}$, E step $0.001 \mathrm{~V}$. Gold electrode is polished for $3 \mathrm{~min}$ with a suspension of 0.3 $\mu \mathrm{m}$ alumina followed by another polishing step for 3 min with a suspension of $0.05 \mu \mathrm{m}$ alumina. Then the polished electrode is sonicated for $10 \mathrm{~min}$ in ethanol and for $10 \mathrm{~min}$ in milliQ water to remove any remaining polishing agent. A second electrochemical cleaning in a $0.5 \mathrm{M} \mathrm{H}_{2} \mathrm{SO}_{4}$ solution is used. Cyclic voltammetry parameters are the following: 10 cycles, scan rate $0.1 \mathrm{~V} / \mathrm{s}$, from -0.2 to $1.6 \mathrm{~V}$, E step $0.001 \mathrm{~V}$. Cyclic voltammetry is repeated until 10 scans are stackable between each other. Finally, the cleaning of the gold electrode is checked by using cyclic voltammetry and electrochemical impedance spectroscopy (Chapter 2).

\section{Immobilization of aptamer via the two-step protocol- separated incubation of 10 $\mu \mathrm{M}$ disulfide aptamers and $1 \mathrm{mM} \mathrm{MCH}$}

All disulfide aptamer solutions are prepared in assembling buffer. A preparation of $100 \mu \mathrm{L}$ is done for each aptamer used. The preparation of $10 \mu \mathrm{M}$ disulfide aptamer solution is achieved as following. $10 \mu \mathrm{L}$ of the aptamer stock $(100 \mu \mathrm{M})$ is mixed with $70 \mu \mathrm{L}$ of assembling buffer then the mixture is heated at $95{ }^{\circ} \mathrm{C}$ for $5 \mathrm{~min}$ and cooled down in an ice bath for $10 \mathrm{~min}$. The solution is spun down for $1 \mathrm{~min}$ at $9000 \mathrm{rpm}$ and stirred for $30 \mathrm{~s}$. The solution needs to reequilibrate at room temperature for $20 \mathrm{~min}$ at least. Then $20 \mu \mathrm{L}$ of DMSO is added to the solution. The mixture is stirred thoroughly and left at RT for 5 h. $60 \mu \mathrm{L}$ of the disulfide aptamer solution is incubated on clean gold electrode for at least $16 \mathrm{~h}$. After $16 \mathrm{~h}$, the modified electrode is washed $3 \mathrm{x}$ with the assembling buffer solution. Then $60 \mu \mathrm{L}$ of $1 \mathrm{mM} \mathrm{MCH}$ in $\mathrm{AB}$ is incubated for $1 \mathrm{~h}$ on the modified gold electrodes. Finally, the modified electrode is washed $5 \mathrm{x}$ with the $\mathrm{AB}$ solution then it is placed in the working solution. After $5 \mathrm{~min}$ in the working solution used, the first EIS measurement can be done. EIS parameters are the following: $E_{D C}$ $0.23 \mathrm{~V}, E_{A C} 0.005 \mathrm{~V}, t_{\text {equilibration }} 4 \mathrm{~s}$, number of frequencies 41 , maximum frequency $10 \mathrm{kHz}$, 
minimum frequency 1.0 Hz. After each EIS measurements, the electrode is removed from the electrolyte solution, it is washed and incubated with the working solution used.

\section{Aptamer density determination via chronocoulometry experiment}

Chronocoulometry experiment must be done at the end of experiments (method that disrupts the SAM formed). Fast amperometry analysis is used for chronocoulometry experiments. The parameters used are the following: $t_{e q} 10 \mathrm{~s}, E_{e q} 0.2 \mathrm{~V}, E_{D C}-0.5 \mathrm{~V}, t_{\text {run }} 0.25 \mathrm{~s}$ and $t_{\text {interval }} 0.01 \mathrm{~s}$. A $3 \mathrm{~mL}$ of $10 \mathrm{mM}$ Tris- $\mathrm{HCl}$ is placed in a conical glass cell. A three electrodes system is used: $\mathrm{Ag} / \mathrm{AgCl}$ as the reference electrode, $\mathrm{Pt}$ wire as the counter electrode and the modified gold $\mathrm{Au}$ ) as the working electrode. The $3 \mathrm{~mL}$ solution is degassed with $\mathrm{N}_{2}$ for $5 \mathrm{~min}$. The first measurement is done with the parameters written above. $Q d l$ is found. Then $15 \mu \mathrm{L}$ of $\mathrm{Ru}\left(\mathrm{NH}_{3}\right)_{6} \mathrm{Cl}_{3}$ is added to the $3 \mathrm{~mL}$ solution, the solution is degassed for $5 \mathrm{~min}$ with $\mathrm{N}_{2}$, the modified gold electrode is incubated in the $3 \mathrm{~mL}$ solution at the same time. The second measurement is taken after 5 min of degassing. Qtotal is found. 


\section{CHAPTER 7}

\section{Conclusion and outlook}

\section{Conclusion}

The objectives of this thesis were set out as follows: (1) unraveling the binding mechanism as well as highlighting the principal binding interactions related to the formation of a MethAptamer complex, (2) using a simple protocol based on the SAM stability monitored for the screening of different electrochemical aptasensor strategies, (3) investigating the SAM stability and developing a new SAM formation protocol to enhance the biorecognition layer stability and the aptamer density and, (4) exploring the different transduced signals of the formed SAM by designing and using control experiments.

The key achievements and contributions of the thesis are discussed below:

A complete characterization of a methamphetamine aptamers family through binding affinity assay, spectroscopic, and calorimetric study has been achieved. The decision process for selecting the optimal aptamer from a pool of four possible molecules required a suitable characterization strategy. Initially, the pool of four aptamers has been narrowed down to one candidate aptamer which has been subsequently characterized in more detail. To narrow down the selected pool of four aptamers to one aptamer, a binding affinity assay and a structural change study have been undertaken. Then, the chosen aptamer has been analyzed with nuclear magnetic resonance (NMR) and isothermal titration calorimetry (ITC) techniques. From these two studies, one of the important nucleotide-binding regions of the aptamer has been enlightened. Furthermore, the thermodynamic parameters of the binding event have been 
observed. A binding mechanism between the aptamer and the methamphetamine has been proposed. Molecular modeling was used to support the binding hypotheses found with the NMR-ITC experiments. The key discovery of this chapter highlights the need of a more suitable technique of SELEX to produce aptamer for small molecule detection. Indeed, the conformational selection binding model and the entropically driven binding event observed create challenges to use the aptamer for the development of an aptamer-based biosensor. Some thoughts to improve the SELEX process are listed in the outlook section.

A stability study has been completed for the major and most suitable electrochemical aptamerbased biosensors existing. The selection has also been done in line with the specific requirements of the project in terms of the level of detection, cost-effectiveness, and simplicity of preparation and use. The aptamer chosen in Chapter $\mathbf{3}$ was used. Before doing any further sensing experiments, the stability of the system in a simple buffer solution must have been controlled. From this stability study, the label-free electrochemical impedance spectroscopy aptasensor has been chosen for further optimization and sensing experiments. In this chapter, one of the key points found is the fact that at our scale of measurement, electrochemical aptamer-based biosensors suffer from a problem of stability. It induces major nonspecific signals that cannot be separated from a potential positive specific signal.

The self-assembled monolayer (SAM) formed by the addition of modified thiolated aptamers on the gold electrode showed some stability limitations by using the electrochemical impedance spectroscopy measurement. Chapter 5 presented a complete investigation of the stability of the formed SAM. Different protocols have been used for enhancing the stability and aptamer density on the electrode surface. Finally, a new protocol using disulfide aptamer has been discovered. The use of the disulfide aptamer is creating a monolayer combining stability and high biorecognition element density on the electrode. A lack of order in the SAM due mainly to the aptamer strand fixation via a 5 'end thiol covalent bonding with gold surface is concluded. The limitation of fixation is overcome with the use of disulfide aptamer that create stability and high aptamer density on the gold electrode surface.

Electrochemical aptamer-based biosensors for small molecule detection are suffering from a significant number of nonspecific interactions that create false positive signals. A complete investigation of our label-free EIS aptasensor system has been achieved through various control experiments in Chapter 6. By using randomized aptamer sequences and testing the response of similar structural molecules to the methamphetamine, we have been able to have a higher 
level of understanding of our label-free EIS aptasensor. For the small molecule detection, enough control experiments must be undertaken. Indeed, a lot of nonspecific interactions between each part of the system have been shown and monitored. The positivity of the signal has to be carefully checked before undertaking other experiments on the aptasensor system.

\section{Outlook}

During the characterization process of the Meth-Aptamer complex, some future perspectives were underlined related to the design of new experiments and the further investigation of the binding model found.

First, as discovered in Chapter 3, the interactions between the Meth molecule and the Aptamer-2 have a lack of specificity. The entropically driven reaction of binding found is specific to a weak interaction between a receptor and its ligand. ${ }^{34}$ To enhance the specificity of the interaction, the bio-engineering of the binding region into the DNA strand, by truncation or addition of specific pseudo-nucleotides, could provide the formation of new hydrogen bonds or covalent bonds between the aptamer and the Meth. For example, a modified pseudonucleotide (the bromodeoxyuracil) can be incorporated instead of a native base in the binding region. The bromodeoxyuracil is activated by the absorption of light resulting in the formation of a bond between the bromodeoxyuracil and an electron-rich amino acid in a target protein. ${ }^{268}$ The replacement of a thymine base by the bromodeoxyuracil can be done in the binding pocket of the Meth-Aptamer, and the resulting binding with the amino group of the Meth may be explored. The covalent bond will afford an irreversible binding event generating a significant specificity to the complex formed and hence, producing a significant biorecognition signal.

A conformational-selection binding model has been proposed in Chapter 3. NMR spectroscopy could provide a method to monitor the conformation-selection equilibrium between the different aptamer conformations. By changing the temperature or the ionic strength of the working solution, the equilibrium between the binding and non-binding state could be controlled. ${ }^{54,164}$

As discovered by Rode et al. ${ }^{269}$, a molecular crowding environment can initiate an induced-fit binding model between an aptamer and its target instead of a conformational-selection binding 
model occurring in a dilute buffer environment. The molecular crowding environment has already been developed for a new SELEX method to obtain more enthalpically driven complex formation between an aptamer and its target. ${ }^{34}$

Finally, the molecular modeling experiments done in Chapter $\mathbf{3}$ are the premise of numerous molecular dynamic experiments. The different conformations of the loop could be investigated, and the addition of methamphetamine molecules could be explored for each of them. The methamphetamine binding to some other parts of the DNA strand could also be explored. The different conformations of the aptamer could be examined by changing the ionic strength of the solution for molecular dynamic experiments and hence, correlated with the results from the NMR experiments stated above.

Some future perspectives concerning the findings of Chapter 5 are described below.

To explore the conformation of the SAM formed with the disulfide protocol, the atomic force microscopy instrument can be used as shown in Josephs et al. ${ }^{121}$.

With the disulfide aptamer, we formed a dense aptamer layer on a gold surface. The purpose was to enhance the e-chem signal produced by the bio-recognition event. Unfortunately, in this study, no specific signal related to the bio-recognition was monitored via EIS. The use of wellstudied aptamers such as thrombin, ${ }^{20}$ cocaine, ${ }^{66}$ or dopamine ${ }^{56}$ could be useful for validating the possible enhancement of the binding signal offers with the disulfide protocol by comparing the results with the thiol protocol.

By keeping the same methamphetamine aptamer, the disulfide protocol could be used with another electrochemical strategy such as the methylene blue non-covalently attached to the DNA strand. Indeed, many aptamers are fixed on the surface, consequently, numerous methylene blue molecules would interact non-covalently with the DNA strands and hence, providing a substantial e-chem signal to monitor.

The chronocoulometry method, usually applied for the aptamer density determination, could also be exploited as a detection method.

Finally, the findings from the Chapter $\mathbf{6}$ can be used for further investigations. 
As shown with the negative aptamer experiment in Chapter 6, a lack concerning the use of negative aptamer to control the system in the aptasensor field is observed. Researches about the design of new control experiments could be done.

As observed with Chapter 6 findings, the use of the backfiller $\mathrm{MCH}$ molecule is not enough to confirm the complete backfilling of the gold surface. It would be useful to develop a new protocol to determine the similarity between the SAM formed and a pure SAM of MCH. As a consequence, it would be possible to obtain a percentage of backfilling for the SAM formed.

The kinetics of adsorption could be investigated to obtain a better understanding of the real interaction occurring for the Meth adsorption on the gold surface.

By using different ionic strengths, it was observed that the adsorption of methamphetamine on a gold surface can be controlled. Some experiments at lower ionic strength than 5.85mM (BWB $2 \mathrm{mM}$ in Chapter 6) could be tested for avoiding methamphetamine adsorption. By contrast, a study could explore a higher ionic strength than $58.5 \mathrm{mM}$ (BWB 20mM in Chapter 6) to see if the methamphetamine adsorption can be monitored in the nanomolar range. 


\section{Appendix}

\begin{tabular}{|l|l|}
\hline Aptamer identification & Sequence 5' $^{\prime} \mathbf{3}^{\prime}$ \\
\hline Aptamer-1 & $\begin{array}{l}\text { ATACGAGCTTGTTCAATAGCGTTTAGGCGTTCATTCATCCCGCTATC } \\
\text { TGGCTGTATCGTGATAGTAAGAGCAATC }\end{array}$ \\
\hline Aptamer-2 & $\begin{array}{l}\text { ATACGAGCTTGTTCAATAGCGTTTCTATCTGGCTGTATCGTGATAGT } \\
\text { AAGAGCACTAATGATAGTAAGAGCAATC }\end{array}$ \\
\hline Aptamer-3 & ATACGAGCTTGTTCAATAGCGTTTAGCGTTCAATTCATCCCGCTATC \\
& CTGGCTGTATCGTGATAGTAGAACAATC \\
\hline Aptamer-4 & ATACGAGCTTGTTCAATAGCGTTTTACGTTCAATTCATCCCGCTATC \\
& TGGCTGTATCGTGATAGTAAGAGCAATC \\
\hline Aptamer-2-40mer & GCGTTTCTATCTGGCTGTATCGTGATAGTAAGAGCACTAA \\
\hline Mutated-Aptamer-2-40mer & GCGTTTCGTCCTGGCTGTATCGTGATAGTAAGAGCACTAA \\
\hline
\end{tabular}

Table A.1. Meth-aptamer sequences used in the study. Buffer conditions are $2 \mathrm{mM}$ Tris- $\mathrm{HCl} \mathrm{pH} \mathrm{7.5,}$ $10 \mathrm{mM} \mathrm{NaCl}, 0.5 \mathrm{mM} \mathrm{KCl}, 0.2 \mathrm{mM} \mathrm{MgCl}_{2}$ and $0.1 \mathrm{mM} \mathrm{CaCl}_{2}$.

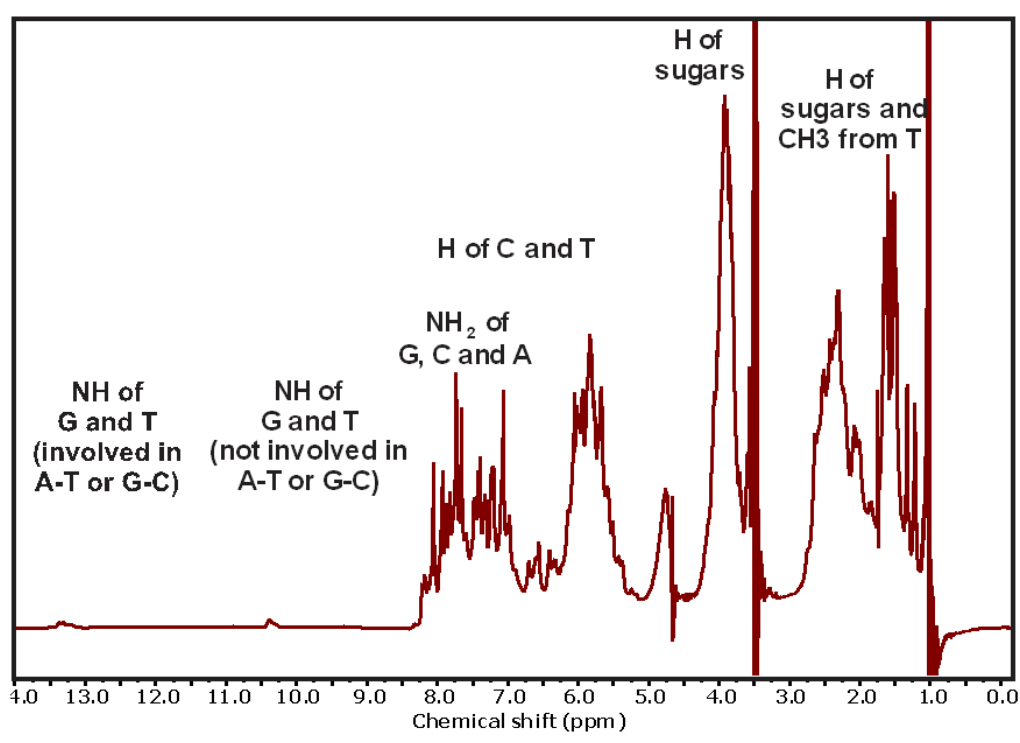

Figure A.1. Aptamer2-40mer ${ }^{1} \mathrm{H}-\mathrm{NMR}$ spectrum. 


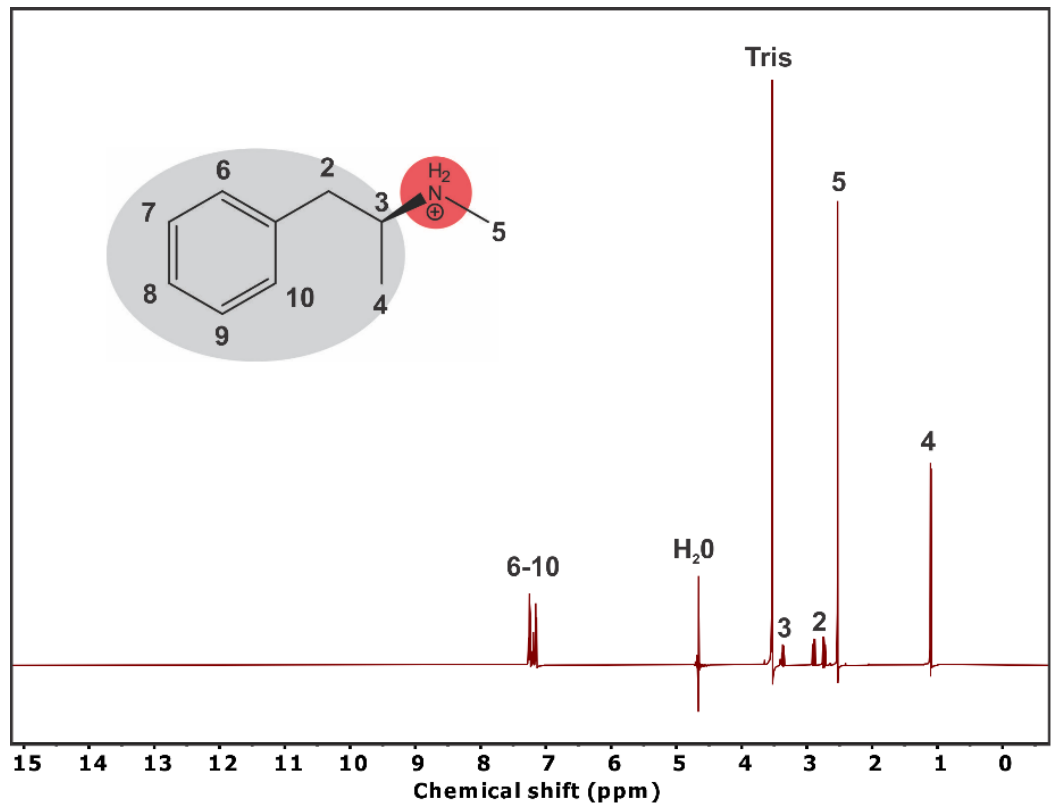

Figure A.2. Meth ${ }^{1} \mathrm{H}-\mathrm{NMR}$ spectrum in BWB $2 \mathrm{mM}$ Tris- $\mathrm{HCl} 90 \%-10 \% \mathrm{D}_{2} \mathrm{O}$.

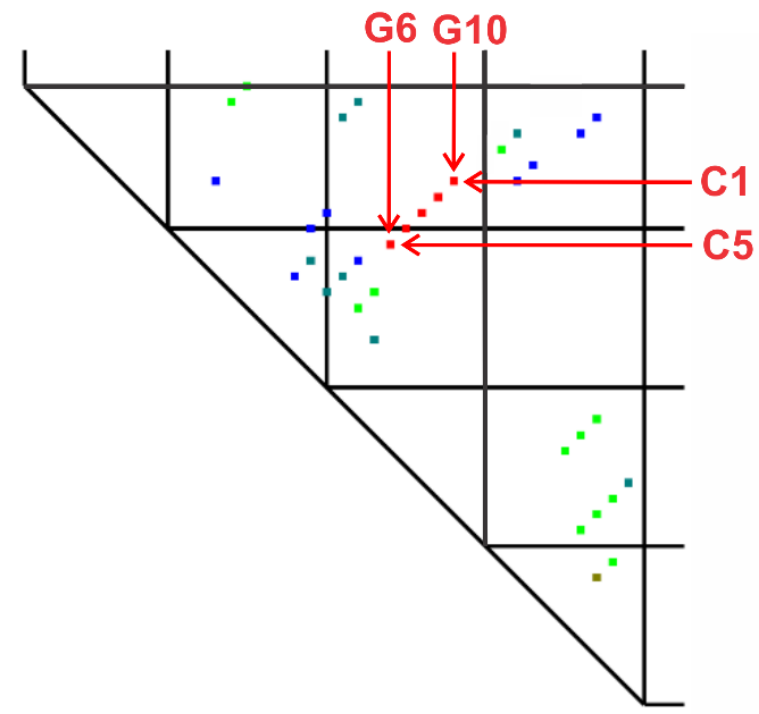

$0.0829963<=-\log 10$ (Probability) < 0.466397

$0.466397<=-\log 10$ (Probability) < 0.849798

$0.849798<=-\log 10$ (Probability) < 1.2332

$1.2332<=-\log 10$ (Probability) $<1.6166$

$1.6166<=-\log 10$ (Probability) $<=2$

partition.pfs

Figure A.3. Partition results for Aptamer2-40mer obtained from RNAstructure. 


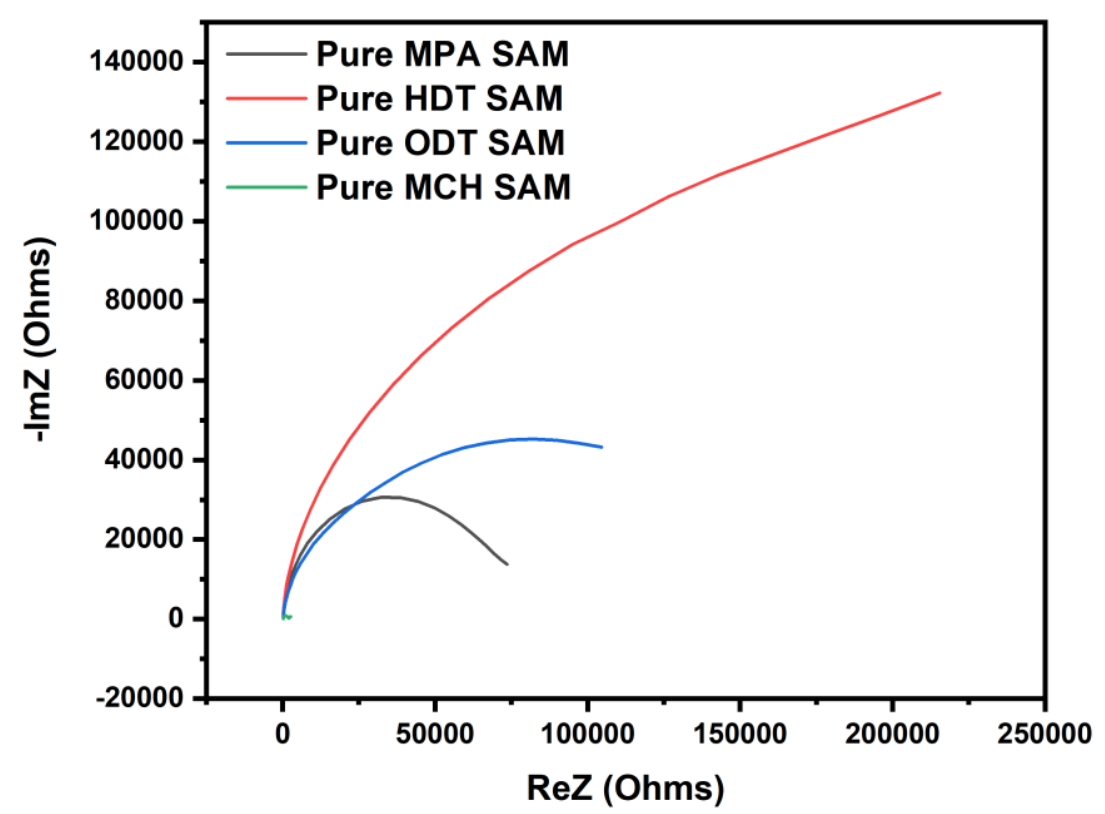

Figure A.4. Initial $R c t$ for different pure blocking agent SAM formed.
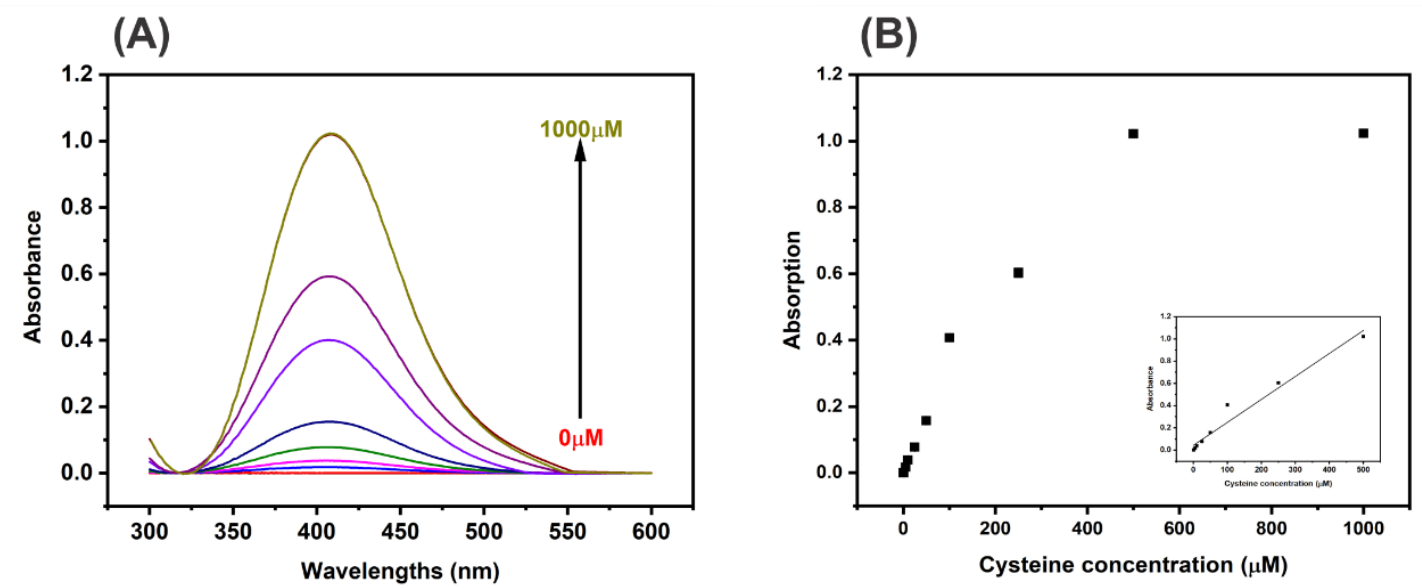

Figure A.5. Ellman's reaction with cysteine. (A) Raw data related to the formation of the TNB molecule proportional to the cysteine concentration. (B) A calibration curve could be achieved by plotting the absorbance at $412 \mathrm{~nm}$ with the cysteine concentration. 


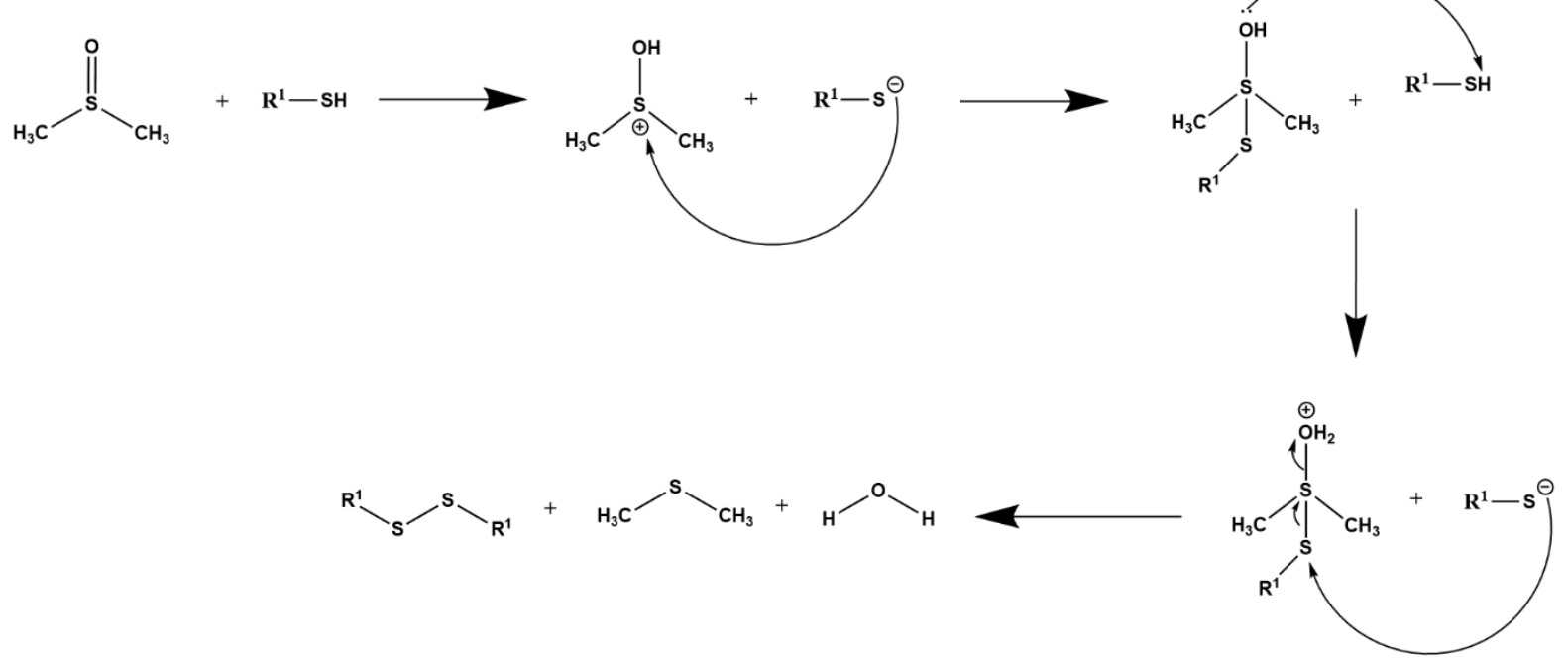

Figure A.6. Thiol oxidation in disulfide mechanism with the dimethylsulfoxide (DMSO) molecule. 


\section{Bibliography}

1. Bhalla, N.; Jolly, P.; Formisano, N.; Estrela, P., Introduction to biosensors. Essays Biochem 2016, 60 (1), 1-8.

2. Turner, A.; Karube, I.; Wilson, G. S., Biosensors : Fundamentals and Applications. 1 ed.; Oxford University Press: Oxford, New York, 1987; $\mathrm{p} 770$.

3. Turner, A. P. F., Biosensors: sense and sensibility. Chemical Society Reviews 2013, 42 (8), 3184-3196.

4. Lowe, C. R., Biosensors. Trends in Biotechnology 1984, 2 (3), 59-65.

5. Sethi, R. S., Transducer aspects of biosensors. Biosensors and Bioelectronics 1994, 9 (3), 243264.

6. Morales, M. A.; Halpern, J. M., Guide to Selecting a Biorecognition Element for Biosensors. Bioconjugate Chemistry 2018, 29 (10), 3231-3239.

7. Scheller, F.; Schubert, F., Biosensors. Elsevier: 1991.

8. Wilson, R.; Turner, A. P. F., Glucose oxidase: an ideal enzyme. Biosensors and Bioelectronics 1992, 7 (3), 165-185.

9. Jarrett, R. J.; Keen, H.; Hardwick, C., "Instant" Blood Sugar Measurement Using Dextrostix and a Reflectance Meter. Diabetes 1970, 19 (10), 724.

10. Cohn, L. A.; McCaw, D. L.; Tate, D. J.; Johnson, J. C., Assessment of five portable blood glucose meters, a point-of-care analyzer, and color test strips for measuring blood glucose concentration in dogs. Journal of the American Veterinary Medical Association 2000, 216 (2), 198-

202.

11. Grudzinskas, J. G.; Jeffrey, D.; Gordon, Y. B.; Chard, T., Specific and sensitive determination of pregnancy-specific $\beta 1$-glycoprotein by radioimmunoassay: A New Pregnancy Test. The Lancet 1977, 309 (8007), 333-335.

12. Crivianu-Gaita, V.; Thompson, M., Aptamers, antibody scFv, and antibody Fab' fragments: An overview and comparison of three of the most versatile biosensor biorecognition elements.

Biosensors and Bioelectronics 2016, 85, 32-45.

13. Iqbal, S. S.; Mayo, M. W.; Bruno, J. G.; Bronk, B. V.; Batt, C. A.; Chambers, J. P., A review of molecular recognition technologies for detection of biological threat agents. Biosensors and Bioelectronics 2000, 15 (11), 549-578.

14. Hidding J. A therapeutic battle: Antibodies vs. Aptamers. Nanosci. Master Progr. 2017;109:120.

15. Jayasena, S. D., Aptamers: An Emerging Class of Molecules That Rival Antibodies in Diagnostics. Clinical Chemistry 1999, 45 (9), 1628-1650.

16. O'Sullivan, C. K., Aptasensors--the future of biosensing? Analytical and Bioanalytical Chemistry 2002, 372 (1), 44-48.

17. Zhou J, Rossi J. Aptamers as targeted therapeutics: current potential and challenges. Nature reviews Drug discovery. 2017 Mar;16(3):181-202.

18. Ellington AD, Szostak JW. In vitro selection of RNA molecules that bind specific ligands. Nature. 1990 Aug;346(6287):818-822.

19. Tuerk, C.; Gold, L., Systematic Evolution of Ligands by Exponential Enrichment: RNA Ligands to Bacteriophage T4 DNA Polymerase. Science 1990, 249 (4968), 505-510.

20. Bock, L. C.; Griffin, L. C.; Latham, J. A.; Vermaas, E. H.; Toole, J. J., Selection of singlestranded DNA molecules that bind and inhibit human thrombin. Nature 1992, 355 (6360), 564-566.

21. Dahm, R., Friedrich Miescher and the discovery of DNA. Developmental Biology 2005, 278

(2), 274-288.

22. Mirsky, A. E., The Discovery of DNA. Scientific American 1968, 218 (6), 78-90. 
23. Watson, J. D.; Crick, F. H. C., Molecular Structure of Nucleic Acids: A Structure for Deoxyribose Nucleic Acid. Nature 1953, 171 (4356), 737-738.

24. Sinden, R. R., CHAPTER 1 - Introduction to the Structure, Properties, and Reactions of DNA. In DNA Structure and Function, Sinden, R. R., Ed. Academic Press: San Diego, 1994; pp 1-57.

25. Sinden, R. R.; Pearson, C. E.; Potaman, V. N.; Ussery, D. W., DNA: Structure and function. In Advances in Genome Biology, Verma, R. S., Ed. JAI: 1998; Vol. 5, pp 1-141.

26. Richards, A. D.; Rodger, A., Synthetic metallomolecules as agents for the control of DNA structure. Chemical Society Reviews 2007, 36 (3), 471-483.

27. Kellett, A.; Molphy, Z.; Slator, C.; McKee, V.; Farrell, N. P., Molecular methods for assessment of non-covalent metallodrug-DNA interactions. Chemical Society Reviews 2019, 48 (4), 971-988.

28. Stoltenburg, R.; Reinemann, C.; Strehlitz, B., SELEX--a (r)evolutionary method to generate high-affinity nucleic acid ligands. Biomolecular Engineering 2007, 24 (4), 381-403.

29. Mosing, R. K.; Mendonsa, S. D.; Bowser, M. T., Capillary Electrophoresis-SELEX Selection of Aptamers with Affinity for HIV-1 Reverse Transcriptase. Analytical Chemistry 2005, 77 (19), 61076112.

30. Stoltenburg, R.; Nikolaus, N.; Strehlitz, B., Capture-SELEX: Selection of DNA Aptamers for Aminoglycoside Antibiotics. Journal of Analytical Methods in Chemistry 2012, 2012, 415697.

31. Gu H, Duan N, Wu S, Hao L, Xia Y, Ma X, Wang Z. Graphene oxide-assisted non-immobilized SELEX of okdaic acid aptamer and the analytical application of aptasensor. Scientific reports. 2016 Feb 22;6(1):1-9.

32. Chatterjee B, Kalyani N, Anand A, Khan E, Das S, Bansal V, Kumar A, Sharma TK. GOLD SELEX: a novel SELEX approach for the development of high-affinity aptamers against small molecules without residual activity. Microchimica Acta. 2020 Nov;187(11):1-3.

33. Fitzwater, T.; Polisky, B., A SELEX primer. In Methods in Enzymology, Academic Press: 1996; Vol. 267, pp 275-301.

34. Huang, M.; Song, J.; Huang, P.; Chen, X.; Wang, W.; Zhu, Z.; Song, Y.; Yang, C., Molecular Crowding Evolution for Enabling Discovery of Enthalpy-Driven Aptamers for Robust Biomedical Applications. Analytical Chemistry 2019, 91 (16), 10879-10886.

35. Du, X.; Li, Y.; Xia, Y.-L.; Ai, S.-M.; Liang, J.; Sang, P.; Ji, X.-L.; Liu, S.-Q., Insights into ProteinLigand Interactions: Mechanisms, Models, and Methods. International journal of molecular sciences 2016, $17(2), 144$.

36. McKeague, M.; De Girolamo, A.; Valenzano, S.; Pascale, M.; Ruscito, A.; Velu, R.; Frost, N. R.; Hill, K.; Smith, M.; McConnell, E. M.; DeRosa, M. C., Comprehensive analytical comparison of strategies used for small molecule aptamer evaluation. Analytical Chemistry 2015, 87 (17), 86088612.

37. Entzian, C.; Schubert, T., Studying small molecule-aptamer interactions using MicroScale Thermophoresis (MST). Methods 2016, 97, 27-34.

38. Amano R, Aoki K, Miyakawa S, Nakamura Y, Kozu T, Kawai G, Sakamoto T. NMR monitoring of the SELEX process to confirm enrichment of structured RNA. Scientific reports. 2017 Mar $21 ; 7(1): 1-9$.

39. Sakamoto, T.; Ennifar, E.; Nakamura, Y., Thermodynamic study of aptamers binding to their target proteins. Biochimie 2018, 145, 91-97.

40. J. Bahnemann, N. B., E. Boschetti, J. G. Bruno,; V. Calzada, B. C., A. Eilers, H. Kaur, G. Perret,; M. Plach, J.-A. P., P. Reich, T. Schubert,; T. K. Sharma, J. W., S. Witt, Aptamers In Biotechnology. In Advances in Biochemical Engineering/Biotechnology, Urmann, K.; Walter, J.-G., Eds. Springer Nature Switzerland AG 2019: 2020; Vol. 174, pp 1-210.

41. Vorlickova, M.; Kejnovska, I.; Bednarova, K.; Renciuk, D.; Kypr, J., Circular dichroism spectroscopy of DNA: from duplexes to quadruplexes. Chirality 2012, 24 (9), 691-698.

42. Wilson, B. D.; Soh, H. T., Re-Evaluating the Conventional Wisdom about Binding Assays. Trends in Biochemical Sciences 2020, 45 (8), 639-649. 
43. Pierce, M. M.; Raman, C. S.; Nall, B. T., Isothermal Titration Calorimetry of Protein-Protein Interactions. Methods 1999, 19 (2), 213-221.

44. Slavkovic, S. J., P.E., Isothermal Titration Calorimetry studies of aptamer small molecule interactions: practicalities and pitfalls. Aptamers 2018, Vol 2, 45-51.

45. Oshima, H.; Yasuda, S.; Yoshidome, T.; Ikeguchi, M.; Kinoshita, M., Crucial importance of the water-entropy effect in predicting hot spots in protein-protein complexes. Physical Chemistry Chemical Physics 2011, 13 (36), 16236-16246.

46. Dunitz, J. D., Win some, lose some: enthalpy-entropy compensation in weak intermolecular interactions. Chemistry \& Biology 1995, 2 (11), 709-712.

47. Fischer, E., Einfluss der Configuration auf die Wirkung der Enzyme. Berichte der deutschen chemischen Gesellschaft 1894, 27 (3), 2985-2993.

48. Tobi, D.; Bahar, I., Structural changes involved in protein binding correlate with intrinsic motions of proteins in the unbound state. Proceedings of the National Academy of Sciences 2005, 102 (52), 18908-18913.

49. Ma, B.; Kumar, S.; Tsai, C. J.; Nussinov, R., Folding funnels and binding mechanisms. Protein Engineering 1999, 12 (9), 713-720.

50. Koshland, D. E., Application of a Theory of Enzyme Specificity to Protein Synthesis. Proceedings of the National Academy of Sciences 1958, 44 (2), 98-104.

51. Csermely P, Palotai R, Nussinov R. Induced fit, conformational selection and independent dynamic segments: an extended view of binding events. Nature Precedings. 2010 May 6:1-1.

52. Hayashi, T.; Oshima, H.; Mashima, T.; Nagata, T.; Katahira, M.; Kinoshita, M., Binding of an RNA aptamer and a partial peptide of a prion protein: crucial importance of water entropy in molecular recognition. Nucleic Acids Research 2014, 42 (11), 6861-6875.

53. Ricci, F.; Vallee-Belisle, A.; Simon, A. J.; Porchetta, A.; Plaxco, K. W., Using Nature's "Tricks" To Rationally Tune the Binding Properties of Biomolecular Receptors. Accounts of Chemical Research 2016, 49 (9), 1884-1892.

54. Bissonnette, S.; Del Grosso, E.; Simon, A. J.; Plaxco, K. W.; Ricci, F.; Vallée-Bélisle, A., Optimizing the Specificity Window of Biomolecular Receptors Using Structure-Switching and Allostery. ACS Sensors 2020, 5 (7), 1937-1942.

55. Vallée-Bélisle, A.; Ricci, F.; Plaxco, K. W., Thermodynamic basis for the optimization of binding-induced biomolecular switches and structure-switching biosensors. Proceedings of the National Academy of Sciences 2009, 106 (33), 13802-13807.

56. Nakatsuka, N.; Yang, K.-A.; Abendroth, J. M.; Cheung, K. M.; Xu, X.; Yang, H.; Zhao, C.; Zhu, B.; Rim, Y. S.; Yang, Y.; Weiss, P. S.; Stojanović, M. N.; Andrews, A. M., Aptamer-field-effect transistors overcome Debye length limitations for small-molecule sensing. Science 2018, 362 (6412), 319-324.

57. Davis, K. A.; Abrams, B.; Lin, Y.; Jayasena, S. D., Use of a High Affinity DNA Ligand in Flow Cytometry. Nucleic Acids Research 1996, 24 (4), 702-706.

58. Feng, C.; Dai, S.; Wang, L., Optical aptasensors for quantitative detection of small biomolecules: A review. Biosensors and Bioelectronics 2014, 59, 64-74.

59. Liu, J., Adsorption of DNA onto gold nanoparticles and graphene oxide: surface science and applications. Physical Chemistry Chemical Physics 2012, 14 (30), 10485-10496.

60. Alsager, O. A.; Kumar, S.; Zhu, B.; Travas-Sejdic, J.; McNatty, K. P.; Hodgkiss, J. M., Ultrasensitive colorimetric detection of 17beta-estradiol: the effect of shortening DNA aptamer sequences. Analytical Chemistry 2015, 87 (8), 4201-4209.

61. Zong, C.; Zhang, Z.; Liu, B.; Liu, J., Adsorption of Arsenite on Gold Nanoparticles Studied with DNA Oligonucleotide Probes. Langmuir 2019, 35 (22), 7304-7311.

62. Zhang F, Liu J. Label-Free Colorimetric Biosensors Based on Aptamers and Gold Nanoparticles: A Critical Review. Analysis \& Sensing. 2021 Feb;1(1):30-43. 
63. Liu, J.; Lu, Y., Fast Colorimetric Sensing of Adenosine and Cocaine Based on a General Sensor Design Involving Aptamers and Nanoparticles. Angewandte Chemie International Edition 2006, 45 (1), 90-94.

64. Gopinath, S. C.; Lakshmipriya, T.; Awazu, K., Colorimetric detection of controlled assembly and disassembly of aptamers on unmodified gold nanoparticles. Biosensors and Bioelectronics 2014, 51, 115-123.

65. Citartan, M.; Gopinath, S. C.; Tominaga, J.; Tan, S. C.; Tang, T. H., Assays for aptamer-based platforms. Biosensors and Bioelectronics 2012, 34 (1), 1-11.

66. Stojanovic, M. N.; de Prada, P.; Landry, D. W., Aptamer-Based Folding Fluorescent Sensor for Cocaine. Journal of the American Chemical Society 2001, 123 (21), 4928-4931.

67. Munzar, J. D.; Ng, A.; Juncker, D., Duplexed aptamers: history, design, theory, and application to biosensing. Chemical Society Reviews 2019, 48 (5), 1390-1419.

68. Villalonga, A.; Pérez-Calabuig, A. M.; Villalonga, R., Electrochemical biosensors based on nucleic acid aptamers. Analytical and Bioanalytical Chemistry 2020, 412 (1), 55-72.

69. Drummond, T. G.; Hill, M. G.; Barton, J. K., Electrochemical DNA sensors. Nature Biotechnology 2003, 21 (10), 1192-1199.

70. Xu, Y.; Cheng, G.; He, P.; Fang, Y., A Review: Electrochemical Aptasensors with Various Detection Strategies. Electroanalysis 2009, 21 (11), 1251-1259.

71. Bard, A. F., L R, Electrochemical methods: fundamentals and applications. Wiley New York: 1980; Vol. 2.

72. Scholz F. Electroanalytical methods. Berlin: Springer; 2010.

73. Zheng, H. Y.; Alsager, O. A.; Wood, C. S.; Hodgkiss, J. M.; Plank, N. O. V., Carbon nanotube field effect transistor aptasensors for estrogen detection in liquids. Journal of Vacuum Science \& Technology B 2015, 33 (6), 06F904.

74. Park, K.; Kwon, S. J.; Kwak, J., A Label-Free Electrochemical Aptasensor for Thrombin Using a Single-Wall Carbon Nanotube (SWCNT) Casted Glassy Carbon Electrode (GCE). Electroanalysis 2014, 26 (3), 513-520.

75. Lin X, Ni Y, Kokot S. Glassy carbon electrodes modified with gold nanoparticles for the simultaneous determination of three food antioxidants. Analytica chimica acta. 2013 Feb 26;765:5462.

76. Li, C.-Z.; Liu, Y.; Luong, J. H. T., Impedance Sensing of DNA Binding Drugs Using Gold Substrates Modified with Gold Nanoparticles. Analytical Chemistry 2005, 77 (2), 478-485.

77. Fan, L.; Zhao, G.; Shi, H.; Liu, M.; Li, Z., A highly selective electrochemical impedance spectroscopy-based aptasensor for sensitive detection of acetamiprid. Biosensors and Bioelectronics 2013, 43, 12-18.

78. Evtugyn G, Porfireva A, Stepanova V, Kutyreva M, Gataulina A, Ulakhovich N, Evtugyn V, Hianik T. Impedimetric aptasensor for ochratoxin A determination based on Au nanoparticles stabilized with hyper-branched polymer. Sensors. 2013 Dec;13(12):16129-16145.

79. Shao, Y.; Wang, J.; Wu, H.; Liu, J.; Aksay, I. A.; Lin, Y., Graphene Based Electrochemical Sensors and Biosensors: A Review. Electroanalysis 2010, 22 (10), 1027-1036.

80. Kaur, N.; Bharti, A.; Batra, S.; Rana, S.; Rana, S.; Bhalla, A.; Prabhakar, N., An electrochemical aptasensor based on graphene doped chitosan nanocomposites for determination of Ochratoxin A. Microchemical Journal 2019, 144, 102-109.

81. Zhu, B.; Alsager, O. A.; Kumar, S.; Hodgkiss, J. M.; Travas-Sejdic, J., Label-free electrochemical aptasensor for femtomolar detection of 17beta-estradiol. Biosensors and Bioelectronics 2015, 70, 398-403.

82. Love, J. C.; Estroff, L. A.; Kriebel, J. K.; Nuzzo, R. G.; Whitesides, G. M., Self-Assembled Monolayers of Thiolates on Metals as a Form of Nanotechnology. Chemical Reviews 2005, 105 (4), 1103-1170.

83. Grönbeck, H.; Curioni, A.; Andreoni, W., Thiols and Disulfides on the Au(111) Surface: The Headgroup-Gold Interaction. Journal of the American Chemical Society 2000, 122 (16), 3839-3842. 
84. Herne, T. M.; Tarlov, M. J., Characterization of DNA Probes Immobilized on Gold Surfaces. Journal of the American Chemical Society 1997, 119 (38), 8916-8920.

85. Häkkinen, H., The gold-sulfur interface at the nanoscale. Nature Chemistry 2012, 4 (6), 443455.

86. Vericat, C.; Vela, M. E.; Benitez, G.; Carro, P.; Salvarezza, R. C., Self-assembled monolayers of thiols and dithiols on gold: new challenges for a well-known system. Chemical Society Reviews 2010, 39 (5), 1805-1834.

87. Wolf, L. K.; Gao, Y.; Georgiadis, R. M., Sequence-Dependent DNA Immobilization: Specific versus Nonspecific Contributions. Langmuir 2004, 20 (8), 3357-3361.

88. Oberhaus, F. V.; Frense, D.; Beckmann, D., Immobilization Techniques for Aptamers on Gold Electrodes for the Electrochemical Detection of Proteins: A Review. Biosensors 2020, 10 (5), 45.

89. Inkpen, M. S.; Liu, Z. F.; Li, H.; Campos, L. M.; Neaton, J. B.; Venkataraman, L., Nonchemisorbed gold-sulfur binding prevails in self-assembled monolayers. Nature Chemistry 2019, 11 (4), 351-358.

90. Pacchioni, G., A not-so-strong bond. Nature Reviews Materials 2019, 4 (4), 226-226.

91. Ying, G.; Wang, M.; Yi, Y.; Chen, J.; Mei, J.; Zhang, Y.; Chen, S., Construction and application of an electrochemical biosensor based on an endotoxin aptamer. Biotechnology and Applied Biochemistry 2018, 65 (3), 323-327.

92. Malvano, F.; Albanese, D.; Pilloton, R.; Di Matteo, M., A new label-free impedimetric aptasensor for gluten detection. Food Control 2017, 79, 200-206.

93. Guo, X.; Wen, F.; Zheng, N.; Luo, Q.; Wang, H.; Wang, H.; Li, S.; Wang, J., Development of an ultrasensitive aptasensor for the detection of aflatoxin B1. Biosensors and Bioelectronics 2014, 56, 340-344.

94. Ma, W.; Yin, H.; Xu, L.; Xu, Z.; Kuang, H.; Wang, L.; Xu, C., Femtogram ultrasensitive aptasensor for the detection of OchratoxinA. Biosensors and Bioelectronics 2013, 42, 545-549. 95. Zhang, K.; Xie, M.; Zhou, B.; Hua, Y.; Yan, Z.; Liu, H.; Guo, L.-n.; Wu, B.; Huang, B., A new strategy based on aptasensor to time-resolved fluorescence assay for adenosine deaminase activity. Biosensors and Bioelectronics 2013, 41, 123-128.

96. Thévenot, D. R.; Toth, K.; Durst, R. A.; Wilson, G. S., Electrochemical biosensors: recommended definitions and classification1International Union of Pure and Applied Chemistry: Physical Chemistry Division, Commission I.7 (Biophysical Chemistry); Analytical Chemistry Division, Commission V.5 (Electroanalytical Chemistry).1. Biosensors and Bioelectronics 2001, 16 (1), 121-131. 97. You, H.; Bai, L.; Yuan, Y.; Zhou, J.; Bai, Y.; Mu, Z., An amperometric aptasensor for ultrasensitive detection of sulfadimethoxine based on exonuclease-assisted target recycling and new signal tracer for amplification. Biosensors and Bioelectronics 2018, 117, 706-712.

98. Xiao, Y.; Lubin, A. A.; Heeger, A. J.; Plaxco, K. W., Label-Free Electronic Detection of Thrombin in Blood Serum by Using an Aptamer-Based Sensor. Angewandte Chemie 2005, 117 (34), 5592-5595.

99. Baker, B. R.; Lai, R. Y.; Wood, M. S.; Doctor, E. H.; Heeger, A. J.; Plaxco, K. W., An Electronic, Aptamer-Based Small-Molecule Sensor for the Rapid, Label-Free Detection of Cocaine in Adulterated Samples and Biological Fluids. Journal of the American Chemical Society 2006, 128 (10), 3138-3139.

100. Hernández, R.; Vallés, C.; Benito, A. M.; Maser, W. K.; Xavier Rius, F.; Riu, J., Graphenebased potentiometric biosensor for the immediate detection of living bacteria. Biosensors and Bioelectronics 2014, 54, 553-557.

101. Zelada-Guillén, G. A.; Tweed-Kent, A.; Niemann, M.; Göringer, H. U.; Riu, J.; Rius, F. X., Ultrasensitive and real-time detection of proteins in blood using a potentiometric carbon-nanotube aptasensor. Biosensors and Bioelectronics 2013, 41, 366-371.

102. Grieshaber D, MacKenzie R, Vörös J, Reimhult E. Electrochemical biosensors-sensor principles and architectures. Sensors. 2008 Mar;8(3):1400-1458. 
103. Macdonald, D. D., Reflections on the history of electrochemical impedance spectroscopy. Electrochimica Acta 2006, 51 (8-9), 1376-1388.

104. Lisdat, F.; Schafer, D., The use of electrochemical impedance spectroscopy for biosensing. Analytical and bioanalytical chemistry 2008, 391 (5), 1555-1567.

105. Jarczewska, M.; Górski, Ł.; Malinowska, E., Electrochemical aptamer-based biosensors as potential tools for clinical diagnostics. Analytical Methods 2016, 8 (19), 3861-3877.

106. Brothers MC, Moore D, St Lawrence M, Harris J, Joseph RM, Ratcliff E, Ruiz ON, Glavin N, Kim SS. Impact of Self-Assembled Monolayer Design and Electrochemical Factors on Impedance-Based Biosensing. Sensors. 2020 Jan;20(8):2246.

107. Rodriguez MC, Kawde AN, Wang J. Aptamer biosensor for label-free impedance spectroscopy detection of proteins based on recognition-induced switching of the surface charge. Chemical Communications. 2005 (34):4267-4269.

108. Neves, M. A. D.; Reinstein, O.; Johnson, P. E., Defining a Stem Length-Dependent Binding Mechanism for the Cocaine-Binding Aptamer. A Combined NMR and Calorimetry Study. Biochemistry 2010, 49 (39), 8478-8487.

109. Neves MA, Reinstein O, Saad M, Johnson PE. Defining the secondary structural requirements of a cocaine-binding aptamer by a thermodynamic and mutation study. Biophysical chemistry. 2010 Dec 1;153(1):9-16.

110. Reinstein, O.; Yoo, M.; Han, C.; Palmo, T.; Beckham, S. A.; Wilce, M. C.; Johnson, P. E., Quinine binding by the cocaine-binding aptamer. Thermodynamic and hydrodynamic analysis of high-affinity binding of an off-target ligand. Biochemistry 2013, 52 (48), 8652-8662.

111. Neves, M. A. D.; Slavkovic, S.; Churcher, Z. R.; Johnson, P. E., Salt-mediated two-site ligand binding by the cocaine-binding aptamer. Nucleic Acids Research 2017, 45 (3), 1041-1048.

112. Lin CH, Patei DJ. Structural basis of DNA folding and recognition in an AMP-DNA aptamer complex: distinct architectures but common recognition motifs for DNA and RNA aptamers complexed to AMP. Chemistry \& biology. 1997 Nov 1;4(11):817-832.

113. Nakamura, I.; Shi, A.-C.; Nutiu, R.; Yu, J. M. Y.; Li, Y., Kinetics of signaling-DNA-aptamer-ATP binding. Physical Review E 2009, 79 (3), 031906.

114. Xia T, Yuan J, Fang X. Conformational dynamics of an ATP-binding DNA aptamer: a singlemolecule study. The Journal of Physical Chemistry B. 2013 Dec 5;117(48):14994-14503.

115. Zhang, Z.; Oni, O.; Liu, J., New insights into a classic aptamer: binding sites, cooperativity and more sensitive adenosine detection. Nucleic Acids Research 2017, 45 (13), 7593-7601.

116. Wang, K. Y.; Krawczyk, S. H.; Bischofberger, N.; Swaminathan, S.; Bolton, P. H., The tertiary structure of a DNA aptamer which binds to and inhibits thrombin determines activity. Biochemistry 1993, 32 (42), 11285-11292.

117. Mao XA, Marky LA, Gmeiner WH. NMR structure of the thrombin-binding DNA aptamer stabilized by Sr2+. Journal of Biomolecular Structure and Dynamics. 2004 Aug 1;22(1):25-33.

118. Jiang, L.; Majumdar, A.; Hu, W.; Jaishree, T. J.; Xu, W.; Patel, D. J., Saccharide-RNA recognition in a complex formed between neomycin B and an RNA aptamer. Structure 1999, 7 (7), 817-S7.

119. Cowan, J. A.; Ohyama, T.; Wang, D.; Natarajan, K., Recognition of a cognate RNA aptamer by neomycin B: quantitative evaluation of hydrogen bonding and electrostatic interactions. Nucleic acids research 2000, 28 (15), 2935-2942.

120. Xu, X.; Makaraviciute, A.; Kumar, S.; Wen, C.; Sjödin, M.; Abdurakhmanov, E.; Danielson, U. H.; Nyholm, L.; Zhang, Z., Structural Changes of Mercaptohexanol Self-Assembled Monolayers on Gold and Their Influence on Impedimetric Aptamer Sensors. Analytical Chemistry 2019, 91 (22), 14697-14704.

121. Josephs, E. A.; Ye, T., Nanoscale Spatial Distribution of Thiolated DNA on Model Nucleic Acid Sensor Surfaces. ACS Nano 2013, 7 (4), 3653-3660.

122. McFadden M, Consultancy DM. The New Zealand drug harm index 2016. Ministry of Health; 2016. 
123. Bardsley A, Low F. Methamphetamine contamination in residential properties: Exposures, risk levels, and interpretation of standards, 2018.

124. Methamphetamine wipe sampling techniques, Hill laboratories.

125. Martyny, J. W.; Arbuckle, S. L.; McCammon, C. S.; Esswein, E. J.; Erb, N.; Van Dyke, M., Chemical concentrations and contamination associated with clandestine methamphetamine laboratories. Journal of Chemical Health \& Safety 2007, 14 (4), 40-52.

126. Standards New Zealand. Testing and Decontamination of Methamphetamine: Contaminated Properties. Standards New Zealand, 2017.

127. Ebrahimi, M.; Johari-Ahar, M.; Hamzeiy, H.; Barar, J.; Mashinchian, O.; Omidi, Y., Electrochemical impedance spectroscopic sensing of methamphetamine by a specific aptamer. Biolmpacts : BI 2012, 2 (2), 91-95.

128. Mao, K.; Yang, Z.; Li, J.; Zhou, X.; Li, X.; Hu, J., A novel colorimetric biosensor based on nonaggregated Au@Ag core-shell nanoparticles for methamphetamine and cocaine detection. Talanta 2017, 175, 338-346.

129. Bishop GR, Chaires JB. Characterization of DNA structures by circular dichroism. Current protocols in nucleic acid chemistry. 2002 Dec;11(1):7-11.

130. Kypr, J.; Kejnovská, I.; Renciuk, D.; Vorlícková, M., Circular dichroism and conformational polymorphism of DNA. Nucleic Acids Research 2009, 37 (6), 1713-1725.

131. Lowry, T. M., Optical rotatory power. Dover publications: 1964.

132. Kelly SM, Jess TJ, Price NC. How to study proteins by circular dichroism. Biochimica et Biophysica Acta (BBA)-Proteins and Proteomics. 2005 Aug 10;1751(2):119-139.

133. Woody RW. Theory of circular dichroism of proteins. In Circular dichroism and the conformational analysis of biomolecules 1996 (pp. 25-67). Springer, Boston, MA.

134. Siligardi, G.; Hussain, R., Circular Dichroism, Applications A2 - Lindon, John C. In Encyclopedia of Spectroscopy and Spectrometry (Third Edition), Tranter, G. E.; Koppenaal, D. W., Eds. Academic Press: Oxford, 2017; pp 293-298.

135. Nagatoishi, S.; Tanaka, Y.; Tsumoto, K., Circular dichroism spectra demonstrate formation of the thrombin-binding DNA aptamer G-quadruplex under stabilizing-cation-deficient conditions.

Biochemical and Biophysical Research Communications 2007, 352 (3), 812-817.

136. Gondeau, C.; Maurizot, J. C.; Durand, M., Circular dichroism and UV melting studies on formation of an intramolecular triplex containing parallel $T^{*} A: T$ and $G * G: C$ triplets: netropsin complexation with the triplex. Nucleic Acids Research 1998, 26 (21), 4996-5003.

137. Fisher, H. F.; Singh, N., Calorimetric methods for interpreting protein-Ligand interactions. In Methods in Enzymology, Academic Press: 1995; Vol. 259, pp 194-221.

138. Amzel, L. M., Loss of translational entropy in binding, folding, and catalysis. Proteins 1997, 28 (2), 144-149.

139. Abraham, R. J.; Fisher, J.; Loftus, P., Introduction to NMR spectroscopy. 1998; Vol. 2.

140. Keeler, J., Understanding NMR spectroscopy. John Wiley \& Sons: 2011.

141. Fürtig, B.; Richter, C.; Wöhnert, J.; Schwalbe, H., NMR Spectroscopy of RNA. ChemBioChem 2003, 4 (10), 936-962.

142. Wijmenga, S. S.; van Buuren, B. N. M., The use of NMR methods for conformational studies of nucleic acids. Progress in Nuclear Magnetic Resonance Spectroscopy 1998, 32 (4), 287-387.

143. Sakamoto T. NMR study of aptamers. Aptamers. 2017;1:13-18.

144. Churcher ZR, Neves MA, Hunter HN, Johnson PE. Comparison of the free and ligand-bound imino hydrogen exchange rates for the cocaine-binding aptamer. Journal of biomolecular NMR. 2017 May;68(1):33-39.

145. Macaya, R. F.; Schultze, P.; Smith, F. W.; Roe, J. A.; Feigon, J., Thrombin-binding DNA aptamer forms a unimolecular quadruplex structure in solution. Proceedings of the National Academy of Sciences 1993, 90 (8), 3745-3749. 
146. Padmanabhan KA, Padmanabhan KP, Ferrara JD, Sadler JE, Tulinsky A. The structure of alpha-thrombin inhibited by a 15-mer single-stranded DNA aptamer. Journal of Biological Chemistry. 1993 Aug 25;268(24):17651-17654.

147. Lin, C. H.; Patel, D. J., Encapsulating an amino acid in a DNA fold. Nature Structural Biology 1996, 3 (12), 1046-1050.

148. Lin, C. H.; Patei, D. J., Structural basis of DNA folding and recognition in an AMP-DNA aptamer complex: distinct architectures but common recognition motifs for DNA and RNA aptamers complexed to AMP. Chemistry \& Biology 1997, 4 (11), 817-832.

149. Zimmermann, G. R.; Jenison, R. D.; Wick, C. L.; Simorre, J. P.; Pardi, A., Interlocking structural motifs mediate molecular discrimination by a theophylline-binding RNA. Nature Structural Biology 1997, 4 (8), 644-649.

150. Jiang, L.; Patel, D. J., Solution structure of the tobramycin-RNA aptamer complex. Nature Structural Biology 1998, 5 (9), 769-774.

151. Grahame, D. C., Mathematical theory of the faradaic admittance. Journal of the Electrochemical Society 1952, 99 (12), 370-385.

152. Biesheuvel, P. M.; Dykstra, J. E., The difference between Faradaic and Nonfaradaic processes in Electrochemistry. arXiv: Chemical Physics 2018.

153. Sawyer, D. T.; Sobkowiak, A.; Roberts, J. L., Electrochemistry for chemists. Wiley: 1995.

154. Hoogvliet, J. C.; Dijksma, M.; Kamp, B.; van Bennekom, W. P., Electrochemical Pretreatment of Polycrystalline Gold Electrodes To Produce a Reproducible Surface Roughness for Self-Assembly:

A Study in Phosphate Buffer pH 7.4. Analytical Chemistry 2000, 72 (9), 2016-2021.

155. Fischer, L. M.; Tenje, M.; Heiskanen, A. R.; Masuda, N.; Castillo, J.; Bentien, A.; Émneus, J.; Jakobsen, M. H.; Boisen, A., Gold cleaning methods for electrochemical detection applications.

Microelectronic Engineering 2009, 86 (4-6), 1282-1285.

156. Imabayashi, SI.; Hobara, D.; Kakiuchi, T.; Knoll, W., Selective Replacement of Adsorbed Alkanethiols in Phase-Separated Binary Self-Assembled Monolayers by Electrochemical Partial Desorption. Langmuir 1997, 13 (17), 4502-4504.

157. Oesch, U.; Janata, J., Electrochemical study of gold electrodes with anodic oxide films-I. Formation and reduction behaviour of anodic oxides on gold. Electrochimica Acta 1983, 28 (9), 12371246.

158. Osteryoung, J. G.; Osteryoung, R. A., Square Wave Voltammetry. Analytical Chemistry 1985, 57 (1), 101A-110A.

159. Chang, B. Y.; Park, S. M., Electrochemical impedance spectroscopy. Annual Review of Analytical Chemistry (Palo Alto Calif) 2010, 3, 207-229.

160. Steel, A. B.; Herne, T. M.; Tarlov, M. J., Electrochemical Quantitation of DNA Immobilized on Gold. Analytical Chemistry 1998, 70 (22), 4670-4677.

161. Jenison, R. D.; Gill, S. C.; Pardi, A.; Polisky, B., High-resolution molecular discrimination by RNA. Science 1994, 263 (5152), 1425-1429.

162. Munzar JD, Ng A, Juncker D. Comprehensive profiling of the ligand binding landscapes of duplexed aptamer families reveals widespread induced fit. Nature communications. 2018 Jan 24;9(1):1-5.

163. Feagin, T. A.; Maganzini, N.; Soh, H. T., Strategies for Creating Structure-Switching Aptamers. ACS Sensors 2018, 3 (9), 1611-1615.

164. Wilson BD, Hariri AA, Thompson IA, Eisenstein M, Soh HT. Independent control of the thermodynamic and kinetic properties of aptamer switches. Nature communications 2019 Nov 7;10(1):1-9.

165. Csermely, P.; Palotai, R.; Nussinov, R., Induced fit, conformational selection and independent dynamic segments: an extended view of binding events. Trends in Biochemical Sciences 2010, 35 (10), 539-546.

166. Hermann, T.; Patel, D. J., Adaptive Recognition by Nucleic Acid Aptamers. Science 2000, 287 (5454), 820-825. 
167. Monod J, Wyman J, Changeux JP. On the nature of allosteric transitions: a plausible model. Journal of Molecular Biology 1965 May 1;12(1):88-118.

168. Cai, S.; Yan, J.; Xiong, H.; Liu, Y.; Peng, D.; Liu, Z., Investigations on the interface of nucleic acid aptamers and binding targets. Analyst 2018, 143 (22), 5317-5338.

169. Zipper, H.; Brunner, H.; Bernhagen, J.; Vitzthum, F., Investigations on DNA intercalation and surface binding by SYBR Green I, its structure determination and methodological implications.

Nucleic Acids Research 2004, 32 (12), e103.

170. Kong, L.; Xu, J.; Xu, Y.; Xiang, Y.; Yuan, R.; Chai, Y., A universal and label-free aptasensor for fluorescent detection of ATP and thrombin based on SYBR Green I dye. Biosensors and Bioelectronics 2013, 42, 193-197.

171. McKeague, M.; Velu, R.; Hill, K.; Bardoczy, V.; Meszaros, T.; DeRosa, M. C., Selection and characterization of a novel DNA aptamer for label-free fluorescence biosensing of ochratoxin $A$. Toxins (Basel) 2014, 6 (8), 2435-2452.

172. Hulme, E. C.; Trevethick, M. A., Ligand binding assays at equilibrium: validation and interpretation. British Journal of Pharmacology 2010, 161 (6), 1219-1237.

173. Bing, T.; Zheng, W.; Zhang, X.; Shen, L.; Liu, X.; Wang, F.; Cui, J.; Cao, Z.; Shangguan, D., Triplex-quadruplex structural scaffold: a new binding structure of aptamer. Scientific Reports 2017, 7 (1), 1-10.

174. Gray DM, Ratliff RL, Vaughan MR. Circular dichroism spectroscopy of DNA. Methods in enzymology. 1992 Jan 1;211:389-406.

175. Li, H.-H.; Wen, C.-Y.; Hong, C.-Y.; Lai, J.-C., Evaluation of aptamer specificity with or without primers using clinical samples for $\mathrm{C}$-reactive protein by magnetic-assisted rapid aptamer selection. RSC Advances 2017, 7 (68), 42856-42865.

176. Zuker, M., Mfold web server for nucleic acid folding and hybridization prediction. Nucleic Acids Research 2003, 31 (13), 3406-3415.

177. Reuter, J. S.; Mathews, D. H., RNAstructure: software for RNA secondary structure prediction and analysis. BMC Bioinformatics 2010, 11 (1), 129.

178. Carlomagno, T., NMR as a tool for structure determination of nucleic acids. EMBL Heidelberg.

179. Mirau, P. A.; Smith, J. E.; Chávez, J. L.; Hagen, J. A.; Kelley-Loughnane, N.; Naik, R., Structured DNA Aptamer Interactions with Gold Nanoparticles. Langmuir 2018, 34 (5), 2139-2146.

180. Leontis, N. B.; Stombaugh, J.; Westhof, E., The non-Watson-Crick base pairs and their associated isostericity matrices. Nucleic Acids research 2002, 30 (16), 3497-3531.

181. Hermann, T.; Westhof, E., Non-Watson-Crick base pairs in RNA-protein recognition. Chemistry \& Biology 1999, 6 (12), R335-R343.

182. Lin, C. H.; Wang, W.; Jones, R. A.; Patel, D. J., Formation of an amino-acid-binding pocket through adaptive zippering-up of a large DNA hairpin loop. Chemistry \& Biology 1998, 5 (10), 555572.

183. Potty, A. S. R.; Kourentzi, K.; Fang, H.; Schuck, P.; Willson, R. C., Biophysical characterization of DNA and RNA aptamer interactions with hen egg lysozyme. International Journal of Biological Macromolecules 2011, 48 (3), 392-397.

184. Archer, W. R.; Schulz, M. D., Isothermal titration calorimetry: practical approaches and current applications in soft matter. Soft Matter 2020, 16 (38), 8760-8774.

185. Gill, D. S.; Roush, D. J.; Willson, R. C., Presence of a preferred anion-exchange binding site on cytochrome b5: structural and thermodynamic considerations. Journal of Chromatography A 1994, $684(1), 55-63$.

186. Zimmermann, G. R.; Wick, C. L.; Shields, T. P.; Jenison, R. D.; Pardi, A., Molecular interactions and metal binding in the theophylline-binding core of an RNA aptamer. RNA 2000, 6 (5), 659-667.

187. Li, H.; Xie, Y.; Liu, C.; Liu, S., Physicochemical bases for protein folding, dynamics, and protein-ligand binding. Science China Life Sciences 2014, 57 (3), 287-302. 
188. Chang, C.-E.; Gilson, M. K., Free Energy, Entropy, and Induced Fit in Host-Guest Recognition: Calculations with the Second-Generation Mining Minima Algorithm. Journal of the American Chemical Society 2004, 126 (40), 13156-13164.

189. Paul, F.; Weikl, T. R., How to Distinguish Conformational Selection and Induced Fit Based on Chemical Relaxation Rates. PLOS Computational Biology 2016, 12 (9), e1005067.

190. Popenda, M.; Szachniuk, M.; Antczak, M.; Purzycka, K. J.; Lukasiak, P.; Bartol, N.; Blazewicz, J.; Adamiak, R. W., Automated 3D structure composition for large RNAs. Nucleic Acids Research 2012, 40 (14), e112-e112.

191. Mathews, D. H., Using an RNA secondary structure partition function to determine confidence in base pairs predicted by free energy minimization. RNA 2004, 10 (8), 1178-1190.

192. Li, S.; Clarkson, M.; McNatty, K., Selection and characterisation of triclosan-specific aptamers using a fluorescence microscope-imaging assay. Analytical and Bioanalytical Chemistry 2020, $412(26), 7285-7294$.

193. Wishart, D. S.; Bigam, C. G.; Yao, J.; Abildgaard, F.; Dyson, H. J.; Oldfield, E.; Markley, J. L.; Sykes, B. D., $1 \mathrm{H}, 13 \mathrm{C}$ and $15 \mathrm{~N}$ chemical shift referencing in biomolecular NMR. Journal of Biomolecular NMR 1995, 6 (2), 135-140.

194. Jeener, J.; Meier, B. H.; Bachmann, P.; Ernst, R. R., Investigation of exchange processes by two-dimensional NMR spectroscopy. The Journal of Chemical Physics 1979, 71 (11), 4546-4553.

195. Piotto, M.; Saudek, V.; Sklenář, V., Gradient-tailored excitation for single-quantum NMR spectroscopy of aqueous solutions. Journal of Biomolecular NMR 1992, 2 (6), 661-665.

196. Willcott, M. R., MestRe Nova. Journal of the American Chemical Society 2009, 131 (36), 13180-13180.

197. Schrödinger LigPrep, New York, NY, 2020.

198. Jones, G.; Willett, P.; Glen, R. C.; Leach, A. R.; Taylor, R., Development and validation of a genetic algorithm for flexible docking. Journal of molecular biology 1997, 267 (3), 727-748.

199. Korb, O.; Stützle, T.; Exner, T. E., Empirical Scoring Functions for Advanced Protein-Ligand Docking with PLANTS. Journal of Chemical Information and Modeling 2009, 49 (1), 84-96.

200. Schrödinger New York, NY, 2019.

201. Kelley, L. A.; Gardner, S. P.; Sutcliffe, M. J., An automated approach for clustering an ensemble of NMR-derived protein structures into conformationally related subfamilies. Protein Engineering, Design and Selection 1996, 9 (11), 1063-1065.

202. Liu, Xin, et al. Electrochemical aptamer sensor for small molecule assays. Chemical Genomics and Proteomics. Humana Press, 2012, 119-132.

203. Yoo, H.; Jo, H.; Oh, S. S., Detection and beyond: challenges and advances in aptamer-based biosensors. Materials Advances 2020, 1 (8), 2663-2687.

204. Farjami, E.; Clima, L.; Gothelf, K. V.; Ferapontova, E. E., DNA interactions with a Methylene Blue redox indicator depend on the DNA length and are sequence specific. Analyst 2010, 135 (6), 1443-1448.

205. Rohs, R.; Sklenar, H.; Lavery, R.; Röder, B., Methylene Blue Binding to DNA with Alternating GC Base Sequence: A Modeling Study. Journal of the American Chemical Society 2000, 122 (12), 2860-2866.

206. Yu, Z.; Luan, Y.; Li, H.; Wang, W.; Wang, X.; Zhang, Q., A disposable electrochemical aptasensor using single-stranded DNA-methylene blue complex as signal-amplification platform for sensitive sensing of bisphenol A. Sensors and Actuators, B: Chemical 2019, 284, 73-80.

207. Wang, J.; Wang, F.; Dong, S., Methylene blue as an indicator for sensitive electrochemical detection of adenosine based on aptamer switch. Journal of Electroanalytical Chemistry 2009, 626 (1-2), 1-5.

208. Fan, C.; Plaxco, K. W.; Heeger, A. J., Electrochemical interrogation of conformational changes as a reagentless method for the sequence-specific detection of DNA. Proceedings of the National Academy of Sciences 2003, 100 (16), 9134-9137. 
209. Schöning MJ, Poghossian A, editors. Label-free biosensing: advanced materials, devices and applications. Springer; 2018 Jul 20.

210. Dubinin AG, Li F, Li Y, Yu J. A solid-state immobilized enzyme polymer membrane microelectrode for measuring lactate-ion concentration. Journal of Electroanalytical Chemistry and Interfacial Electrochemistry. 1991 Feb 1;320(1):131-135.

211. Shul'ga, A. A.; Koudelka-Hep, M.; de Rooij, N. F.; Netchiporouk, L. I., Glucose-sensitive enzyme field effect transistor using potassium ferricyanide as an oxidizing substrate. Analytical chemistry 1994, 66 (2), 205-210.

212. Leasen, S.; Sritunyalucksana-Dangtip, K.; Hodak, J. H.; Srisala, J.; Kulsing, C.; Veerasia, W., Using Electrochemical Impedance Spectroscopy of Methylene Blue and Ferricyanide for DNA Sensing Surface Characterization. In Chemistry for Sustainable Development, Gupta Bhowon, M.; JhaumeerLaulloo, S.; Li Kam Wah, H.; Ramasami, P., Eds. Springer Netherlands: Dordrecht, 2012; pp 249-264. 213. Banasiak, A.; Colleran, J., Determination of Integrity, Stability and Density of the DNA Layers Immobilised at Glassy Carbon and Gold Electrodes Using Ferrocyanide. Electroanalysis 2020, 32 (10), 2220-2230.

214. Lin, Z.; Chen, L.; Zhang, G.; Liu, Q.; Qiu, B.; Cai, Z.; Chen, G., Label-free aptamer-based electrochemical impedance biosensor for 17beta-estradiol. Analyst 2012, 137 (4), 819-822.

215. Bogomolova, A.; Komarova, E.; Reber, K.; Gerasimov, T.; Yavuz, O.; Bhatt, S.; Aldissi, M., Challenges of Electrochemical Impedance Spectroscopy in Protein Biosensing. Analytical Chemistry 2009, 81 (10), 3944-3949.

216. Dauphin-Ducharme, P.; Plaxco, K. W., Maximizing the Signal Gain of Electrochemical-DNA Sensors. Analytical Chemistry 2016, 88 (23), 11654-11662.

217. White, R. J.; Phares, N.; Lubin, A. A.; Xiao, Y.; Plaxco, K. W., Optimization of Electrochemical Aptamer-Based Sensors via Optimization of Probe Packing Density and Surface Chemistry. Langmuir 2008, 24 (18), 10513-10518.

218. Wink, T.; J. van Zuilen, S.; Bult, A.; P. van Bennekom, W., Self-assembled Monolayers for Biosensors. Analyst 1997, 122 (4), 43R-50R.

219. Nuzzo, R. G.; Allara, D. L., Adsorption of bifunctional organic disulfides on gold surfaces. Journal of the American Chemical Society 1983, 105 (13), 4481-4483.

220. Bain, C. D.; Whitesides, G. M., Molecular-Level Control over Surface Order in Self-Assembled Monolayer Films of Thiols on Gold. Science 1988, 240 (4848), 62-63.

221. Rouhana, L. L.; Moussallem, M. D.; Schlenoff, J. B., Adsorption of Short-Chain Thiols and Disulfides onto Gold under Defined Mass Transport Conditions: Coverage, Kinetics, and Mechanism. Journal of the American Chemical Society 2011, 133 (40), 16080-16091.

222. Xu, D.; Xu, D.; Yu, X.; Liu, Z.; He, W.; Ma, Z., Label-Free Electrochemical Detection for Aptamer-Based Array Electrodes. Analytical Chemistry 2005, 77 (16), 5107-5113.

223. Xu, Y.; Yang, L.; Ye, X.; He, P.; Fang, Y., An Aptamer-Based Protein Biosensor by Detecting the Amplified Impedance Signal. Electroanalysis 2006, 18 (15), 1449-1456.

224. Kafka, J.; Pänke, O.; Abendroth, B.; Lisdat, F., A label-free DNA sensor based on impedance spectroscopy. Electrochimica Acta 2008, 53 (25), 7467-7474.

225. Keighley, S. D.; Li, P.; Estrela, P.; Migliorato, P., Optimization of DNA immobilization on gold electrodes for label-free detection by electrochemical impedance spectroscopy. Biosensors and Bioelectronics 2008, 23 (8), 1291-1297.

226. Miodek, A.; Regan, E. M.; Bhalla, N.; Hopkins, N. A.; Goodchild, S. A.; Estrela, P., Optimisation and Characterisation of Anti-Fouling Ternary SAM Layers for Impedance-Based Aptasensors. Sensors (Basel) 2015, 15 (10), 25015-25032.

227. Zhang, F.; Cho, S. S.; Yang, S. H.; Seo, S. S.; Cha, G. S.; Nam, H., Gold Nanoparticle-Based Mediatorless Biosensor Prepared on Microporous Electrode. Electroanalysis 2006, 18 (3), 217-222.

228. Lazar, J.; Schnelting, C.; Slavcheva, E.; Schnakenberg, U., Hampering of the Stability of Gold Electrodes by Ferri-/Ferrocyanide Redox Couple Electrolytes during Electrochemical Impedance Spectroscopy. Analytical Chemistry 2016, 88 (1), 682-687. 
229. Vogt, S.; Su, Q.; Gutierrez-Sanchez, C.; Noll, G., Critical View on Electrochemical Impedance Spectroscopy Using the Ferri/Ferrocyanide Redox Couple at Gold Electrodes. Analytical Chemistry 2016, 88 (8), 4383-4390.

230. Schreiber, F., Self-assembled monolayers: from simple model systems to biofunctionalized interfaces. Journal of Physics: Condensed Matter 2004, 16 (28), R881-R900.

231. Murphy, J. N.; Cheng, A. K. H.; Yu, H.-Z.; Bizzotto, D., On the Nature of DNA Self-Assembled Monolayers on Au: Measuring Surface Heterogeneity with Electrochemical in Situ Fluorescence Microscopy. Journal of the American Chemical Society 2009, 131 (11), 4042-4050.

232. Ricci, F.; Lai, R. Y.; Heeger, A. J.; Plaxco, K. W.; Sumner, J. J., Effect of Molecular Crowding on the Response of an Electrochemical DNA Sensor. Langmuir 2007, 23 (12), 6827-6834.

233. Peterson, A. W.; Heaton, R. J.; Georgiadis, R. M., The effect of surface probe density on DNA hybridization. Nucleic Acids Research 2001, 29 (24), 5163-5168.

234. Xu, S.; Laibinis, P. E.; Liu, G.-y., Accelerating the Kinetics of Thiol Self-Assembly on GoldA Spatial Confinement Effect. Journal of the American Chemical Society 1998, 120 (36), 9356-9361.

235. Liu, Y. Aptasensors: from fundamentals to new sensor concepts. Victoria University of Wellington, 2019.

236. David, M., Avoid Misrepresenting Data. 2019.

237. Smith, T., The hydrophilic nature of a clean gold surface. Journal of Colloid and Interface Science 1980, 75 (1), 51-55.

238. Aliakbarinodehi, N.; Jolly, P.; Bhalla, N.; Miodek, A.; De Micheli, G.; Estrela, P.; Carrara, S., Aptamer-based Field-Effect Biosensor for Tenofovir Detection. Scientific Reports 2017, 7, 1-10.

239. Xu, S.; Cruchon-Dupeyrat, S. J. N.; Garno, J. C.; Liu, G.-Y.; Kane Jennings, G.; Yong, T.-H.; Laibinis, P. E., In situ studies of thiol self-assembly on gold from solution using atomic force microscopy. The Journal of Chemical Physics 1998, 108 (12), 5002-5012.

240. Mehennaoui, S.; Poorahong, S.; Jimenez, G. C.; Siaj, M., Selection of high affinity aptamerligand for dexamethasone and its electrochemical biosensor. Scientific Reports 2019, 9 (1), 1-9.

241. Bain, C. D.; Biebuyck, H. A.; Whitesides, G. M., Comparison of self-assembled monolayers on gold: coadsorption of thiols and disulfides. Langmuir 1989, 5 (3), 723-727.

242. Tyndall, G. S.; Ravishankara, A. R., Atmospheric oxidation of reduced sulfur species. International Journal of Chemical Kinetics 1991, 23 (6), 483-527.

243. Taube, H., Mechanisms of oxidation with oxygen. Journal of General Physiology 1965, 49 (1), 29-52.

244. Yiannios, C. N.; Karabinos, J. V., Oxidation of Thiols by Dimethyl Sulfoxide. The Journal of Organic Chemistry 1963, 28 (11), 3246-3248.

245. Sun, J.-G.; Weng, W.-Z.; Li, P.; Zhang, B., Dimethyl sulfoxide as a mild oxidant in S-P(O) bond construction: simple and metal-free approaches to phosphinothioates. Green Chemistry 2017, 19 (4), 1128-1133.

246. Tam, J. P.; Wu, C. R.; Liu, W.; Zhang, J. W., Disulfide bond formation in peptides by dimethyl sulfoxide. Scope and applications. Journal of the American Chemical Society 1991, 113 (17), 66576662.

247. Wallace, T. J., Reactions of Thiols with Sulfoxides. I. Scope of the Reaction and Synthetic Applications. Journal of the American Chemical Society 1964, 86 (10), 2018-2021.

248. Barany, G.; Angell, Y. M.; Annis, I.; Chen, L.; Gross, C. M.; Hargittai, B., Current strategies for disulfide bridge formation in synthetic peptides and proteins. Innovation and Perspectives in Solid Phase Synthesis \& Combinatorial Libraries 1998, 85-88.

249. Ellman, G. L., A colorimetric method for determining low concentrations of mercaptans. Arch Biochem Biophys 1958, 74 (2), 443-450.

250. Simpson, R. J., Estimation of Free Thiols and Disulfide Bonds Using Ellman's Reagent. CSH Protoc 2008, pdb.prot4699.

251. Szucs, A., On the Adsorption of Glucose Oxidase at a Gold Electrode. Journal of The Electrochemical Society 1989, 136 (12), 3748. 
252. Lipkowski, J.; Shi, Z.; Chen, A.; Pettinger, B.; Bilger, C., lonic adsorption at the Au(111) electrode. Electrochimica Acta 1998, 43 (19), 2875-2888.

253. Chen, A.; Lipkowski, J., Electrochemical and Spectroscopic Studies of Hydroxide Adsorption at the $\mathrm{Au}(111)$ Electrode. The Journal of Physical Chemistry B 1999, 103 (4), 682-691.

254. Kerner, Z.; Pajkossy, T., Measurement of adsorption rates of anions on Au(111) electrodes by impedance spectroscopy. Electrochimica Acta 2002, 47 (13), 2055-2063.

255. Moulton, S. E.; Barisci, J. N.; Bath, A.; Stella, R.; Wallace, G. G., Investigation of protein adsorption and electrochemical behavior at a gold electrode. Journal of Colloid and Interface Science 2003, 261 (2), 312-319.

256. Zakaria, H. M.; Shah, A.; Konieczny, M.; Hoffmann, J. A.; Nijdam, A. J.; Reeves, M. E., Small Molecule- and Amino Acid-Induced Aggregation of Gold Nanoparticles. Langmuir 2013, 29 (25), 7661-7673.

257. Zong, C.; Liu, J., The Arsenic-Binding Aptamer Cannot Bind Arsenic: Critical Evaluation of Aptamer Selection and Binding. Analytical Chemistry 2019, 91 (16), 10887-10893.

258. Miodek, A.; Regan, E. M.; Bhalla, N.; Hopkins, N. A. E.; Goodchild, S. A.; Estrela, P., Optimisation and Characterisation of Anti-Fouling Ternary SAM Layers for Impedance-Based Aptasensors. Sensors 2015, 15 (10), 25015-25032.

259. Loo, A. H.; Bonanni, A.; Pumera, M., Impedimetric thrombin aptasensor based on chemically modified graphenes. Nanoscale 2012, 4 (1), 143-147.

260. Contreras Jimenez, G.; Eissa, S.; Ng, A.; Alhadrami, H.; Zourob, M.; Siaj, M., Aptamer-based label-free impedimetric biosensor for detection of progesterone. Analytical Chemistry 2015, 87 (2), 1075-1082.

261. Wang, Y.; Feng, J.; Tan, Z.; Wang, H., Electrochemical impedance spectroscopy aptasensor for ultrasensitive detection of adenosine with dual backfillers. Biosensors and Bioelectronics 2014, $60,218-223$.

262. Zhu B, Booth MA, Woo HY, Hodgkiss JM, Travas-Sejdic J. Label-free, electrochemical quantitation of potassium ions from femtomolar levels. Chemistry-An Asian Journal. 2015 Oct;10(10):2169-2175.

263. Zhou, A.; Xie, Q.; Wu, Y.; Cai, Y.; Nie, L.; Yao, S., Study of the Adsorption of Glutathione on a Gold Electrode by Using Electrochemical Quartz Crystal Impedance, Electrochemical Impedance Spectroscopy, and Cyclic Voltammetry. Journal of Colloid and Interface Science 2000, 229 (1), 12-20. 264. Möller, J.; Schroer, M. A.; Erlkamp, M.; Grobelny, S.; Paulus, M.; Tiemeyer, S.; Wirkert, F. J.; Tolan, M.; Winter, R., The effect of ionic strength, temperature, and pressure on the interaction potential of dense protein solutions: from nonlinear pressure response to protein crystallization. Biophysical journal 2012, 102 (11), 2641-2648.

265. Willey, J. D., The Effect of Ionic Strength on the Solubility of an Electrolyte. Journal of Chemical Education 2004, 81 (11), 1644.

266. MacKenzie, R. G.; Heischober, B., Methamphetamine. Pediatrics in Review 1997, 18 (9), 305309.

267. Kamalakannan, S.; Prakash, M.; Chambaud, G.; Hochlaf, M., Adsorption of Hydrophobic and Hydrophilic lonic Liquids at the Au(111) Surface. ACS Omega 2018, 3 (12), 18039-18051.

268. Mukhopadhyay, R., Aptamers are ready for the spotlight. ACS Publications: 2005.

269. Rode, A. B.; Endoh, T.; Sugimoto, N., Crowding Shifts the FMN Recognition Mechanism of Riboswitch Aptamer from Conformational Selection to Induced Fit. Angewandte Chemie International Edition 2018, 57 (23), 6868-6872. 